\title{
IMPORTANT THERAPEUTIC TARGETS IN ACUTE PANCREATITIS
}

Ph.D. Thesis

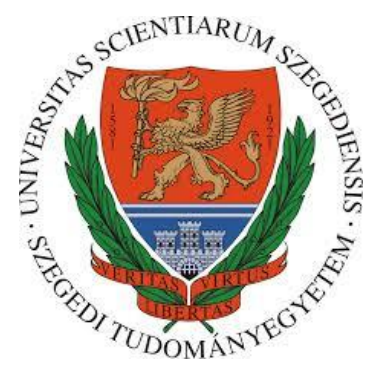

Emese Tóth

Supervisors:

Prof. Péter Hegyi M.D., Ph.D., D.Sc. , MAE 1,2,3

József Maléth M.D., Ph.D. ${ }^{2,4}$

${ }^{1}$ Momentum Translational Gastroenterology Research Group, Hungarian Academy of Sciences-University of Szeged, Szeged, Hungary

${ }^{2}$ First Department of Medicine, University of Szeged, Szeged, Hungary

${ }^{3}$ Institute for Translational Medicine and First Department of Medicine, University of Pécs, Pécs, Hungary,

${ }^{4}$ Momentum Epithelial Cell Signalling and Secretion Research Group, Hungarian Academy of Sciences-University of Szeged, Szeged, Hungary

\section{Szeged}




\section{TABLE OF CONTENTS}

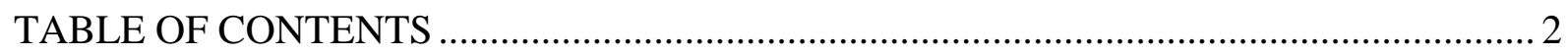

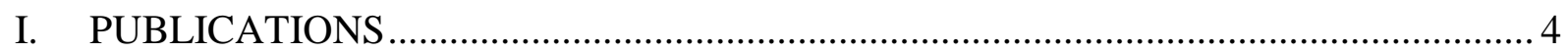

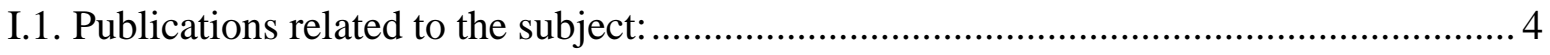

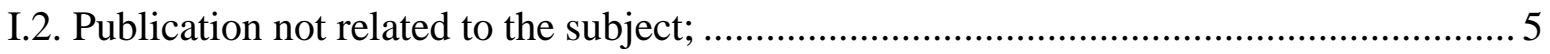

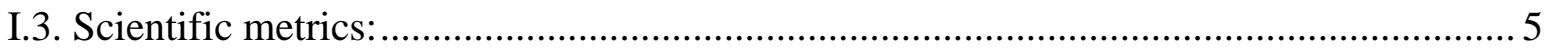

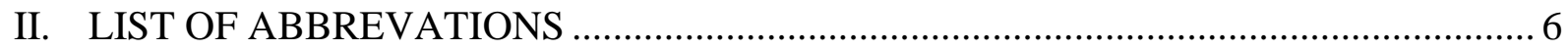

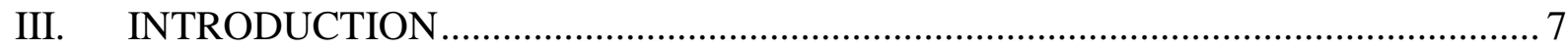

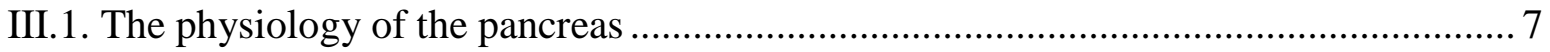

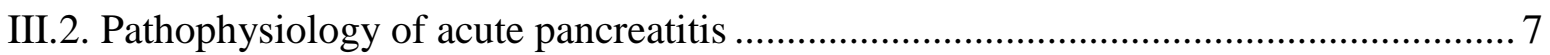

III.3. Targeting the mitochondrial transition pore as potential therapeutic target in AP .... 8

III.4. Importance of pancreatic ductal fluid secretion ................................................... 9

III.5. Alteration between acid-base balance and AP .................................................. 10

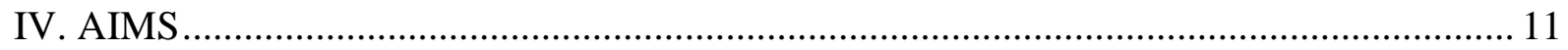

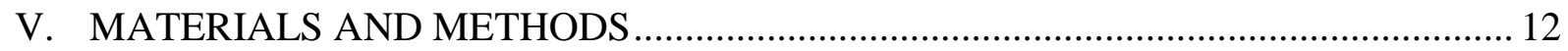

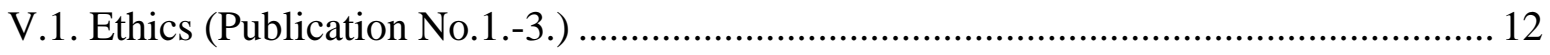

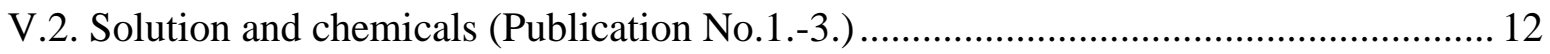

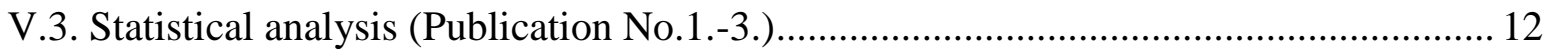

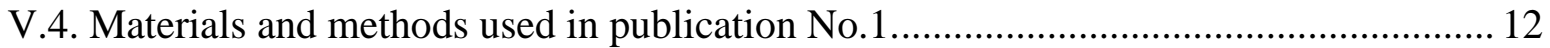

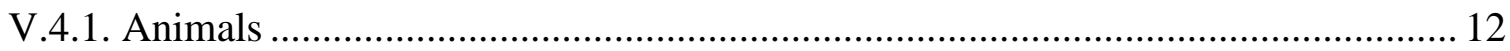

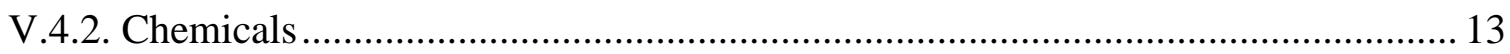

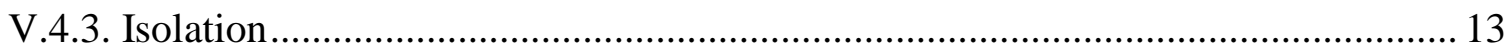

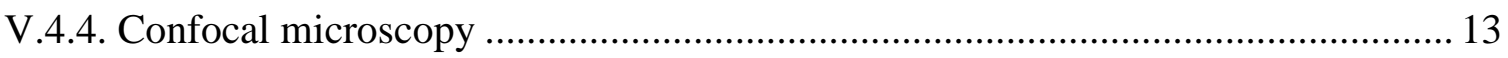

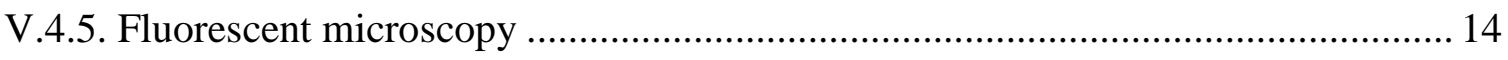

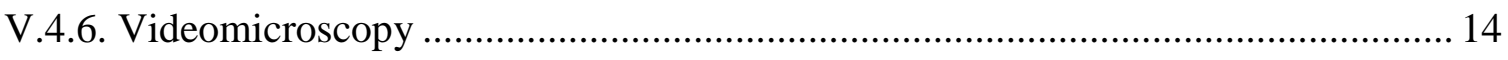

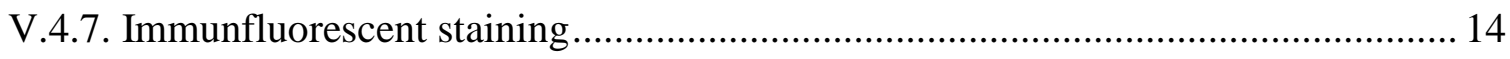

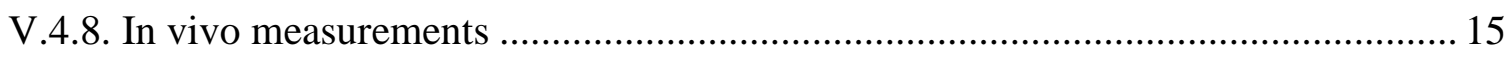

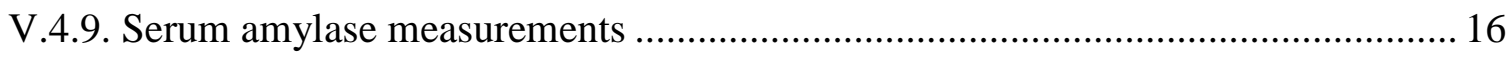

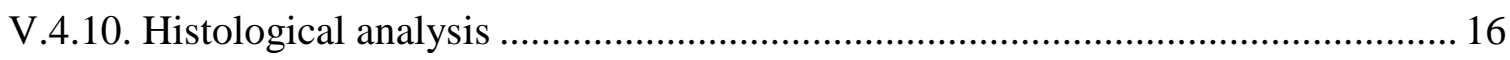

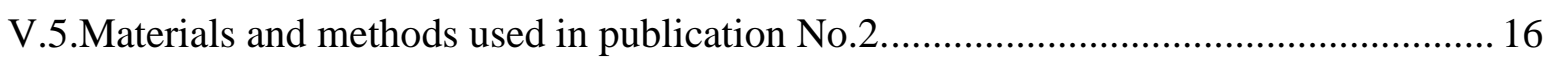

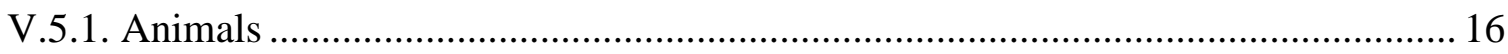

V.5.2. Immunfluorescent stainings and detection of AQP1 and CFTR channels in mouse

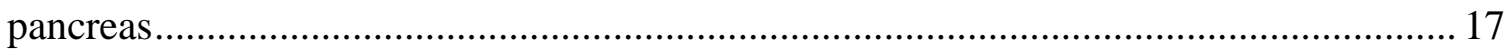

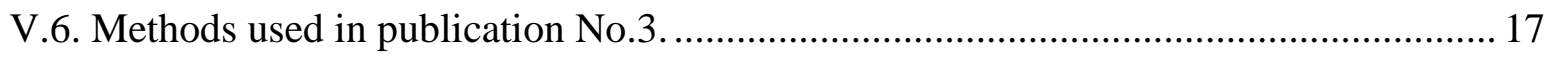




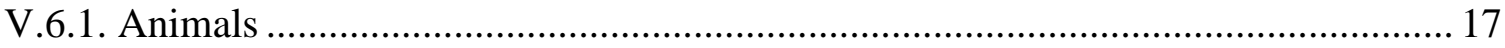

V.6.2. Development of the new model of MA in mice.................................................... 18

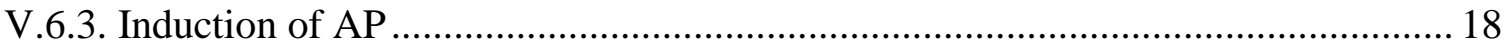

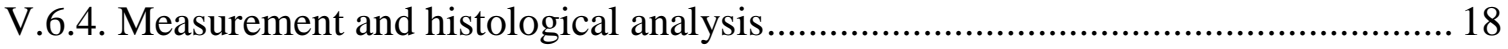

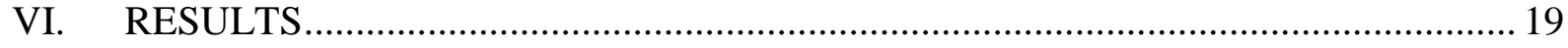

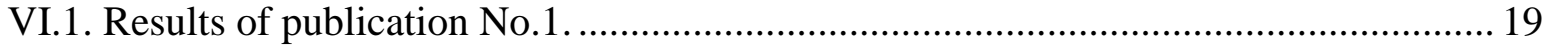

VI.1.1. Genetic inhibition of mPTP protects mitochondrial homeostasis and cell function evoked by pancreatitis-inducing factors in PDEC ....................................................... 19

VI.1.2. Pharmacological inhibition of mPTP by CyA effectively prevents mitochondrial damage evoked by pancreatitis-inducing factors in PDEC ............................................. 21

VI.1.3. NIM811 treatment protects mitochondrial function and bicarbonate transport mechanisms in PDEC

VI.1.4. Pharmachological inhibition of mPTP have no effects on pancreatic ductal fluid

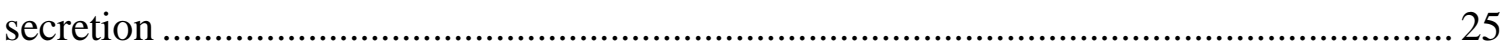

VI.1.5. NIM811 treatment protects mitochondrial function in acinar cells ...................... 26

VI.1.6. NIM811 reduces the severity of AP in mice....................................................... 28

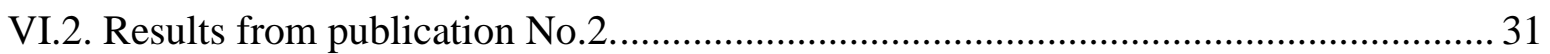

VI.2.1. Aquaporin1 and CFTR expression in mouse pancreas ..................................... 31

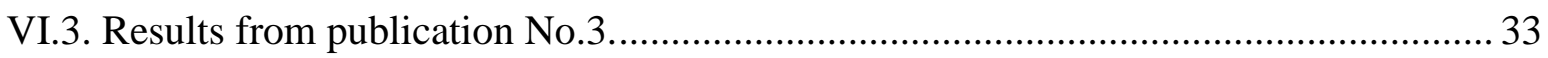

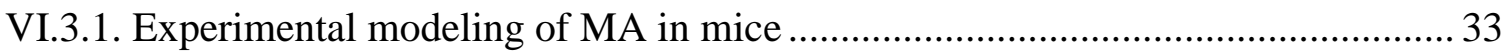

VI.3.2. Pre-existing Acidosis Deteriorates Both Mild and Severe Forms of AP in Mice 34

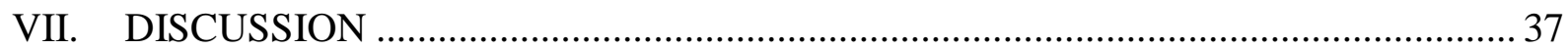

VII.1. Protecting the mitochondrial homeostasis as a novel therapeutic option in AP-

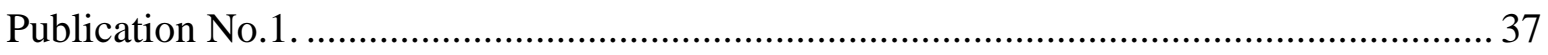

VII.2. The role of AQP1 in pancreatic ductal fluid secretion- Publication No.2 ............... 38

VII.3. The vicious cycle between reduced blood $\mathrm{pH}$ and AP-Publication No.3.................. 38

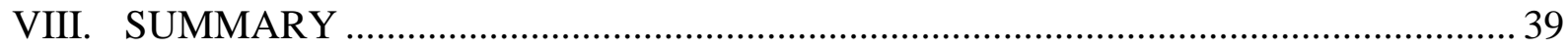

VIII.1. Conclusions, new therapeutic options in the treatment of AP ............................... 39

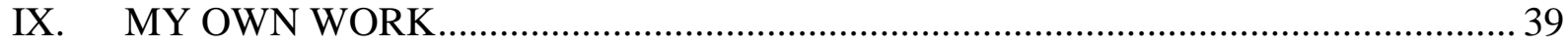

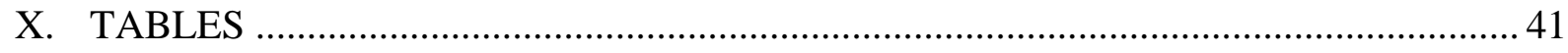

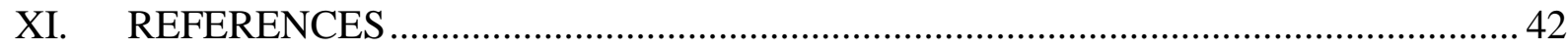

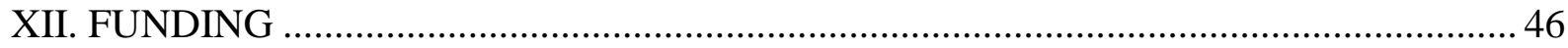

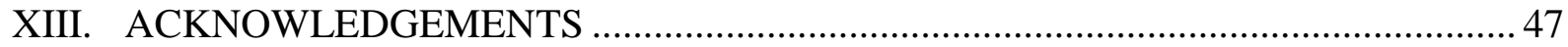




\section{PUBLICATIONS}

\section{I.1. Publications related to the subject:}

\section{Publication No.1.;}

Emese Tóth, József Maléth, Noémi Závogyán,Júlia Fanczal, Anna Grassalkovich, Réka Erdős, Petra Pallagi, Gergő Horváth, László Tretter, Emese Réka Bálint, Zoltán Rakonczay Jr., Viktória Venglovecz, Péter Hegyi "Novel mitochondrial transition pore inhibitor N-methyl-4-isoleucine cyclosporin is a new therapeutic option in acute pancreatitis"

The Journal of Physiology (2019 in press)

Original publication , IF: 4.98, Q1

\section{Publication No.2.;}

Viktória Venglovecz, Petra Pallagi, Lajos V. Kemény, Anita Balázs, Zsolt Balla, Eszter Becskeházi, Eleonóra Gál, Emese Tóth, Ágnes Zvara, László G. Puskás, Katalin Borka, Matthias Sendler, Markus M. Lerch, Julia Mayerle, Jens-Peter Kühn, Zoltán Rakonczay, Jr., and Péter Hegyi "The Importance of Aquaporin 1 in Pancreatitis and Its Relation to the CFTR Cl- Channel. "

Frontiers in physiology ( 2018) doi: 10.3389/fphys.2018.00854

Original publication, IF: 3.394, Q2

\section{Publication No.3.;}

Zoltan Rumbus*, Emese Toth*, Laszlo Poto, Aron Vincze, Gabor Veres, Laszlo Czako, Emoke Olah, Katalin Marta, Alexandra Miko, Zoltan Rakonczay, Jr., Zsolt Balla, Jozsef Kaszaki, Imre Foldesi, Jozsef Maleth, Peter Hegyi", and Andras Garami" "Bidirectional Relationship Between Reduced Blood $\mathrm{pH}$ and Acute Pancreatitis: A Translational Study of Their Noxious Combination"

Frontiers in physiology ( 2018) doi: 10.3389/fphys.2018.01360

Original publication, IF: 3.394, Q2

*Authors share a co-authorship of this article, "Authors share a co- last authorship of this article 


\section{I.2. Publication not related to the subject;}

Andrea Szentesi, Emese Tóth, Emese Bálint, Júlia Fanczal, Tamara Madácsy, Dorottya Laczkó, Imre Ignáth, Anita Balázs, Petra Pallagi, József Maléth, Zoltán Rakonczay, Jr, Balázs Kui, Dóra Illés, Katalin Márta, Ágnes Blaskó, Alexandra Demcsák, Andrea Párniczky, Gabriella Pár, Szilárd Gódi, Dóra Mosztbacher, Ákos Szücs, Adrienn Halász, Ferenc Izbéki, Nelli Farkas, Péter Hegyi, and Hungarian Pancreatic Study Group “Analysis of Research Activity in Gastroenterology: Pancreatitis Is in Real Danger"

Plos One (2016) doi: 10.1371/journal.pone.0165244

Original publication, IF: 3.057, D1

\section{I.3. Scientific metrics:}

\begin{tabular}{|c|c|}
\hline $\begin{array}{l}\text { Number of publications: } \\
\text { Cummulative impact factor: }\end{array}$ & $\begin{array}{l}4 \text { (2 first authors) } \\
14.825\end{array}$ \\
\hline $\begin{array}{l}\text { Number of total citations ( Google Scholar) } \\
\text { https://scholar.google.com/citations?hl=hu\&user=esjT }\end{array}$ & 24 \\
\hline $\mathrm{pS}$, Hirsch index & 2 \\
\hline $\begin{array}{l}\text { Number of total citations (MTMT2) } \\
\text { https://m2.mtmt.hu/gui2/?type=authors\&mode=brows }\end{array}$ & 19 \\
\hline e\&sel, Hirsch index & 2 \\
\hline
\end{tabular}




\section{LIST OF ABBREVATIONS}

AP- acute pancreatitis,

NIM811- N-metil-izoleucine cyclosporine,

mPTP- mitochondrial transition pore, mitochondrial membrane potencial- $\psi$,

CFTR- cystic fibrosis transmembrane conductance regulator,

PDEC-pancreatic ductal epithelial cells,

Cyclophylin D- Cyp D,

CYA- cylosporin A,

Hepatitis C virus-HCV,

Debio025- Alispovirir,

Tro40303-3,5-seco-4-nor-cholestan-5-one-oxime-3-o,

TMRM- Tetramethylrhodamine Methyl Ester Perchlorate,

TOM20- Mitochondrial import receptor subunit,

FA- fatty acid (palmitoliec acid),

FAEE- Fatty acid ethyl ester,

ETOH- ethanol,

BA- CDC- chenodeoxycholic acid,

BCECF-AM (2',7'-Bis-(2-Carboxyethyl)-5-(and-6)-Carboxyfluorescein, Acetoxymethyl Ester),

CER-caerulein,

TAU- sodium taurocholate,

TBS- Tris Buffered Solution,

BSA- Bovine Serum Albumin,

HBSS- Hank1s Stock Solution,

CBD- Common Pancreatic Biliary Duct,

CCCP- Carbonyl cyanide 3-chlorophenylhydrazone

CP-Chronic panreatitis

AQP-aquaporin channel

MA-metabolic acidosis

MAP- mild acute pancreatitis

SAP- severe acute pancreatitis 


\section{INTRODUCTION}

\section{III.1. The physiology of the pancreas}

The pancreas is a heterocrine gland which has endocrine and digestive exocrine function as well. It is a major organ of the digestive system, it functions as an exocrine gland by secreting pancreatic juice into the duodenum. The endocrine function of the gland is that , it regulates blood sugar levels, by secreting hormones such as insulin, glucagon, somatostatin and pancreatic polypeptide. The exocrine part of the pancreas include the acinar and ductal system. As an exocrine organ, the pancreas secretes $1.5-21$ of fluid daily ${ }^{[1,2]}$ which contains the inactive form of digestive enzymes and is high in $\mathrm{HCO}_{3}$ - which is produced by the pancreatic ductal cells ${ }^{[2]}$. The main functions of this high in $\mathrm{HCO}_{3}$ - fluid are:

- to provide an alkaline environment in the duodenum for the optimal function of digestive enzymes ${ }^{[3]}$

- to wash out the toxic factors (such as bile acids) from the ducts ${ }^{[4]}$ to prevent the premature activation of zymogens ${ }^{[5]}$

\section{III.2. Pathophysiology of acute pancreatitis}

One of the leading gastrointestinal disorders which requires hospitalization in the United States is acute pancreatitis (AP) ${ }^{[6-8]}$. AP is generally mild, but in severe forms the mortality rate is unacceptably high $(28 \%)^{[9]}$. Specific treatment of the patients is still unsolved, the current clinical therapy relies on fluid replacement, jejunal, or parenteral nutrition and intensive care in severe cases. AP is one of the most challenging gastrointestinal disorders for several reasons:

- its development is not fully understood ${ }^{[10,11]}$

- it has no specific therapy ${ }^{[10,11]}$

- the incidence rate of the disease is continuously increasing ${ }^{[12]}$

- The mortality of the disease is extremely high ${ }^{[9]}$

Henceforth, recently several investigations have been published concerning the risk factors of AP and chronic pancreatitis (CP);

- obesity, hyperlipidemia and hypertension are independent risk factors for a few complications in $\mathrm{AP}^{[13]}$

- timing and optimization of ERCP indication in biliary AP patients is critical ${ }^{\text {[14] }}$

- pancreatitis-associated mortality is more common with advanced age ${ }^{[15]}$ 
- Comorbidities determine mortality whereas both comorbidities and aging predict severity of AP ${ }^{[16]}$

- Body-mass index correlates with severity and mortality in $\mathrm{AP}^{[17]}$

- The presence of hypertriglyceridemia worsens the course and outcome of AP ${ }^{[18]}$

- Preexisting diabetes elevates risk of local and systemic complications in $\mathrm{AP}^{[19]}$

- Misfolding cationic trypsinogen variant p.L104P causes hereditary pancreatitis ${ }^{[20]}$

Also, there have been some recent studies which were focusing on the details of AP treatment ; centralized care for acute pancreatitis significantly improves outcomes of the disease ${ }^{[21]}, \mathrm{C}$ reactive protein (CRP) within $24 \mathrm{~h}$ from the onset of pain is an inclusion criterion elevates event rates and reduces the number of patients required in trials on $\mathrm{AP}^{[22]}$ and patients with suspected infection because of fever has no benefit from antibiotic therapy ${ }^{[23]}$. Moreover, the Goulash plus trial will examine risk and influencing factors leading to $\mathrm{CP}$ and identify the most useful measurable parameters. ${ }^{[24]}$

However, much less attention have been made on new therapeutic targets. AP is a multifactorial disease ${ }^{[10,11]}$ involving several types of cells, including acinar and ductal cells. Concerning the molecular mechanism involved in the disease, it is known that the followings have important roles:

- trypsinogen activation ${ }^{[11,25]}$

- impaired autophagy $[11,25]$

- excessive Ca2+ influx $[11,25,26]$

- calcineurin activation $^{[11,25]}$

- mitochondrial dysfunction ${ }^{[11,25]}$

- cystic fibrosis transmembrane conductance regulator (CFTR) inhibition ${ }^{[11,25]}$

Therefore, targeting one of these could lead to the first specific therapy in AP.

\section{III.3. Targeting the mitochondrial transition pore as potential therapeutic target in AP}

Mitochondrial dysfunction is one of the earliest events in the disease ${ }^{[27-30]}$. It has been revealed, that in acinar cells bile acids (BA) and ethanol and fatty acids (EtOH+FA) open the membrane transition pore (mPTP) channel via the cyclophilin D (Cyp D) subunit, and by keeping the channel opened mitochondrial depolarization, lower ATP synthesis and cell necrosis occur ${ }^{[29 \text {, }}$ 31, 32]. Yet, it is still a mystery how pancreatic ductal epithelial cells (PDEC) are affected. Nowadays, to experimentally inhibit mPTP (via Cyp D) cyclosporin A (CyA) is the only licenced compound ${ }^{[33]}$. However, the clinical use of CyA is questionable: 
- A trial found that CyA could reduce the size and damage of myocardial infarction, but larger studies showed no beneficial effects ${ }^{[33-35]}$.

- Debio025 (a CyA derivative, Alispovirir, Debiopharm) has been found useful against hepatitis $\mathrm{C}$ virus (HCV), but surprisingly, some of the patients developed pancreatitis, which ended up in a clinical hold on the global Debio025 trials ${ }^{[36,37]}$.

- TRO40303 (3,5-seco-4-nor-cholestan-5-one oxime-3-o, TROPHOS, Roche) is another mPTP inhibitor and it was not beneficial in a phase 2 trial of cardiac preservation following acute myocardial infarction, questioning its effectivity ${ }^{[33,38,39]}$.

- Both Debio025 and TRO40303 have been described as useful in experimental models, but due to the clinical failures they did not reached higher levels of clinical trials in AP.

- Recently, a novel CyA A derivative; $N$-methyl-4-isoleucine cyclosporin (NIM811), was shown to be greatly beneficial in different experimental and clinical studies ${ }^{[40-45]}$. No toxicity or severe or serious adverse effects have been reported in the studies in which NIM811 were used, suggesting that it does not have severe immunosuppressant activity either ${ }^{[46]}$.

\section{III.4. Importance of pancreatic ductal fluid secretion}

As mentioned before, clinical and experimental studies indicate that impaired ductal $\mathrm{HCO}_{3}-$ secretion makes the pancreas more susceptible to inflammatory diseases such as AP or chronic pancreatitis $(\mathrm{CP})^{[25,47-50]}$. Interestingly, the available data about the pancreatic ductal water transport processes are much less than what is known about pancreatic ductal $\mathrm{HCO}_{3}$ - secretion, except the general fact that the movement of electrolytes is osmotically coupled to water flow. It is assumed by numerous studies that there is a physical interaction between the CFTR Clchannel and certain aquaporin (AQP) isoforms ${ }^{[51-53]}$ Henceforth, colocalization of this two channel has been revealed in the human pancreas ${ }^{[54]}$. AQP1 is the major water channel of human red blood cells and in the digestive system the main result of AQP1 deletion is manifested in serum hypotriglyceridemia and steatorrhea with higher stool trygliceride concentration and increased lipase activity ${ }^{[55,56]}$.In the peritoneum the lack of AQP1 ends up in significantly reduced osmotical water transport. ${ }^{[55,57-59]}$ Howewer, there is only a few data available about AQPs in the pancreas and how these channels interact with other channels of the pancreatic ducts. During our study we aimed to characterize the pathophysiological and pathological role of AQPs in the pancreatic ductal secretion, one part of my dissertation focuses on the expression and possible interaction between CFTR and AQP1 channels in pancreatic ducts. 


\section{III.5. Alteration between acid-base balance and AP}

AP is often co-occurred by alterations in the acid-base balance, however, how changes of blood $\mathrm{pH}$ influences the outcome of AP is still unknown. Acidosis is often considered as a marker of disease severity ${ }^{[60]}$. It is known that when pancreatic bicarbonate production is altered by local or systemic acid load (metabolic acidosis, MA), the resulting lower $\mathrm{pH}$ can trigger pancreatic enzyme activation and deteriorate cell damage ${ }^{[61]}$. Moreover, injection of acidic contrast solution into the pancreatic duct increased the severity of experimental AP in rats ${ }^{[62,63]}$. Takács et al. have shown that in patients with AP the luminal $\mathrm{pH}$ of the main pancreatic duct was also lower compared to control human samples ${ }^{[48]}$.

These suggest that may the development of AP is coupled with the decrease of local $\mathrm{pH}$. It is important to mention, that there are some mechanisms which can take place during AP and can result in MA, for example during the occurrence of drainage or pancreatic fistula the $\mathrm{HCO}_{3}-$ rich pancreatic juice could be lost ${ }^{[64]}$, or lactic acidosis could be manifested. ${ }^{[65]}$ Lactic acidosis could happen during AP for example because of shock, sepsis, cardiovascular failure, or upper gastrointestinal bleeding ${ }^{[65]}$. Sadly, the interaction between AP and systemic $\mathrm{pH}$ is still not fully clarified. During our study we developed a new mouse model of chronic metabolic acidosis (MA) and induced mild (MAP) or severe (SAP) AP in the mice to study the alterations between the diseases. During our study, we measured laboratory parameters and quantified the extent of pancreatic edema, necrosis, and leukocyte. The discovery of how the metabolic acidosis affect the outcome of AP in animals could open new therapeutic ways in the treatment of AP. ${ }^{[66]}$ 


\section{AIMS}

\section{I. (Publication No.1.):}

a.) Pancreatitis inducing factors open the membrane transition pore (mPTP) channel via cyclophilin D activation in acinar cells causing calcium overload and cell death. Notably, there is still no available data from how pancreatic ductal epithelial cells are affected by mPTP inhibition. Therefore, we aimed to investigate how genetic and pharmacological inhibition of mPTP affects the function of pancreatic ductal epithelial cells.

b.) Genetic or pharmacological inhibition of $\mathrm{mPTP}$ improves the outcome of acute pancreatitis in animal models. Howewer, clinical testing of different mPTP inhibitors were stopped before reaching the "proof of concept" phase 2 clinical trials due to severe problems of their effectiveness and/or safety. Thereby, we aimed to test the novel Cyclosporin A derivative NIM811 during in vivo animal experiments.

\section{II. (Publication No.2.):}

Decreased pancreatic ductal fluid secretion plays a critical role in AP. Therefore, our aim was to study the mechanisms and function of aquaporins which are involved in transepithelial water flow movements in epithelial fluid secretion in several types of tissues.

Specific aim: To investigate the presence of AQP1 water and CFTR ion channels in mouse pancreatic tissue slices.

\section{III. (Publication No.3.):}

Acid-base abnormality is common in acute pancreatitis (AP). Lowering extracellular $\mathrm{pH}$ deteriorates the manifestation of $\mathrm{AP}$ in rats and decrease of luminal $\mathrm{pH}$ in the pancreas contributes to the tissue damage in AP in mice. Hence, our aim was to study effect of metabolic acidosis during the manifestation of AP in mice.

Specific aim I.: To develop a mouse model of metabolic acidosis in mice

Specific aim II: To study the effect of metabolic acidosis on experimental AP. 


\section{MATERIALS AND METHODS}

\section{V.1. Ethics (Publication No.1.-3.)}

The animal experiments were performed in compliance with European Union Directive 2010/63/EU and Hungarian Government Decree 40/2013 (II.14.). Experiments were approved by local ethics committees for investigations involving animals at the University of Szeged . In our studies all animals were euthanized by $200 \mathrm{mg} / \mathrm{kg}$ pentobarbital i.p. (Bimeda MTC, Cambridge, Canada).

\section{V.2. Solution and chemicals (Publication No.1.-3.)}

All chemicals were obtained from Sigma-Aldrich (Budapest, Hungary), unless otherwise stated. 2.7-bis-(2-carboxyethyl)-5-(and-6-) carboxyfluorescein-acetoxymethylester (BCECF-AM) and Tetramethylrhodamine-methylester (TMRM) were purchased from Termofischer Scientific. NIM811 were purchased from MedChem Express Europe (Sweden). Cyclosporin A (CYA), caerulein (CER), NIM811, CCCP and fluorescence dies were diluted in dimethyl sulfoxide (DMSO) . Table 1 describes the constitution of solutions that we used during the study.

\section{V.3. Statistical analysis (Publication No.1.-3.)}

All data are expressed as means \pm SEM. Analysis were performed by either one- or two-way analysis of variance (ANOVA) or Kruskal-Wallis tests followed by the Holm-Sidak Method as appropriate (Sigma Plot). The effects were considered significant when $\mathrm{p}<0.05$.

\section{V.4. Materials and methods used in publication No.1.}

\section{V.4.1. Animals}

A total of 70 wild type (WT) and cyclophilin D knockout (Cyp D KO, (B6;129-Ppiftm1Maf/J) mice were sacrificed. Cyp $D K O$ mice were generated by targeted disruption of the Ppif gene (which encodes the Cyp D that is a component of the mPTP). ${ }^{[67]}$ Cyp D KO animals were provided for us by the Department of Medical Biochemistry, Semmelweis University, Budapest, Hungary. Wild type and Cyp D-deficient littermate mice (of C57Bl/6 J background, either sex, aged between 20 and 45 days) were housed in a room maintained at $20-22^{\circ} \mathrm{C}$ on a $12 \mathrm{~h}$ light-dark cycle with food and water available ad libitum. To ensure a homologous genetic background, mice were backcrossed with C57B16/J mice for at least eight generations. 


\section{V.4.2. Chemicals}

In this study $500 \mu \mathrm{M}$ Chenodeoxycholic acid (bile acid,BA) or $100 \mathrm{mM}$ ethanol (EtOH) + $200 \mu \mathrm{M}$ palmitoleic acid (fatty acid, FA) was used during the fluorescence, confocal microscopy and immunostaining measurements, to evaluate the effect of bile acids or the alcohol and fatty acid induced damage on the mitochondrial and cell function during the genetic or pharmachological inhibition of the mPTP in pancreatic ducts or acinar cells. $100 \mu \mathrm{M}$ of Carbonyl cyanide 3-chlorophenylhydrazone (CCCP) were used in the mitochondrional measurements as a positive control for mitochondrial damage. $2 \mu \mathrm{M}$ CYA and $2 \mu \mathrm{M}$ NIM811 were used to pharmacologically inhibit mPTP. Prior to the fluorescence and confocal microscopy, immunostainings, the cells (ducts and acinar cells as well) from the CYA- or NIM811- treated groups were pretreated for 25-30 minutes with the compounds (CYA or NIM811).

\section{V.4.3. Isolation}

Isolation of pancreatic ducts and acinar cells were performed by microdissection and enzymatic digestion as described earlier. ${ }^{[68,69]}$ Briefly, intra/interlobular ducts were isolated by enzymatic digestion and microdissection from the pancreas and cultured overnight.

\section{V.4.4. Confocal microscopy}

Mitochondrial membrane potential ( $\Psi)$ were determined by Zeiss LSM 880 confocal laser scanning microscope (Carl Zeiss Technika Kft., Budaörs, Hungary). BA or EtOH + FA were used to induce mitochondrial damage. Isolated pancreatic ducts or acinar cells were incubated in standard HEPES solution and loaded with TMRM (Tetrame thylrhodamine Methyl Ester Perchlorate , $100 \mathrm{nmol} / \mathrm{L})$.

In order to monitor apoptotic and necrotic cells in isolated pancreatic ducts or acinar cells an apoptosis/necrosis kit was used (ab176750, Abcam). To determinate live, necrotic or apoptotic cells, CytoCalcein Violet 450 fluorescent, Apopxin Deep Red Indicator and Nuclear Green DCS1 fluorecence dies (ab176750, Abcam) were used. Samples were incubated in the mixture of the above stated fluorescence dyes at room temperature for 30-35 mins (after 25 min treatment of with $\mathrm{BA} / \mathrm{ETOH}+\mathrm{FA} / \mathrm{CYA} / \mathrm{NIM} 811$ ) in dark prior to the confocal microscopy measuremets. In case of CYA or NIM811 treated ducts or acinar cells, the incubation with these compounds were performed before staining with the fluorescence dyes. Stainings were analyzed using a Zeiss LSM 880 confocal laser scanning microscope (Carl Zeiss Technika Kft., Budaörs, Hungary). Live, necrotic or apoptotic cells were counted and summarized in 
percentage of each sample, then data were summarized to average and statistical analysis was performed.

\section{V.4.5. Fluorescent microscopy}

Microfluorometry was used to measure pancreatic ductal $\mathrm{HCO}_{3}{ }^{-}$secretion as described earlier [70, 71] by using BCECF-AM (2',7'-Bis-(2-Carboxyethyl)-5-(and-6)Carboxyfluorescein, Acetoxymethyl Ester, $1.5 \mathrm{mmol} / \mathrm{L})$.

\section{V.4.6. Videomicroscopy}

In vitro pancreatic ductal fluid secretion (luminal swelling) assays were developed by Fernández-Salazar et al, ${ }^{[72]}$ performed by videomicroscopy as described earlier ${ }^{[73]}$. Briefly, stimulaton of pancreatic ductal fluid secretion was induced by $5 \mu \mathrm{M}$ forskolin and $100 \mu \mathrm{M} 3$ isobutyl-1-methylxanthine (IBMX), quantification were performed by Image J Software [73]. In vivo fluid secretion measurements were performed on anesthetized (by i.p. $87.5 \mathrm{mg} / \mathrm{kg}$ ketamine-12.5 mg/kg xylazine) mice after CER or EtOH+FA induced AP prior to euthanasia. Animals were placed on warm pads $(37 \circ \mathrm{C})$ to maintain the body temperature. Briefly, the abdomen of the mice were opened and cannucaltion of the lumen of the common biliopancreatic duct was performed by a 30 -gauge needle ${ }^{[50]}$. Then the proximal end of the common duct was closed by a microvessel clip (Braun-Aesculap, Tuttlingen, Germany) to prevent contamination with bile, and the pancreatic juice was collected in PE-10 tube for $15 \mathrm{~min}$. In vivo secretion was induced by i.p. administration of $0.75 \mathrm{CU} / \mathrm{kg}$ secretin ${ }^{[50]}$.

\section{V.4.7. Immunfluorescent staining}

Mitochondria were detected with immunofluorescent staining (TOM20 mitochondrial marker, (EPR15581-39, Abcam)). In order to determine mitochondrial localization in isolated pancreatic ductal or acinar cells we labeled the mitochondria by the using of TOM20 primary antibody (Abcam, EPR15581-39). TOM20 is the central unit of the receptor TOM complex in the mitochondrial outer membrane and the role of it is to recognize and translocate cytosolically synthetized mitochondrial preproteins ${ }^{[74-76]}$ Isolated pancreatic ducts were frozen in cryomold at $20^{\circ} \mathrm{C}$. The cryosections (thickness $7 \mu \mathrm{m}$ ) of the isolated pancreatic ducts from WT and Cyp D KO mice were cut by Leica Cryostat. Sections were fixed in $4 \%$ paraformaldehyde. Washing periods were administered with 1xTBS solution. Antigen retrieval was performed with $10 \mathrm{mM}$ Sodium -Citrate solution at the $\mathrm{pH}$ of 6 at $95{ }^{\circ} \mathrm{C}$ for 15 minutes. Blocking was obtained for $1 \mathrm{~h}$ with $1 \%$ goat serum in $5 \%$ BSA-TBS solution. After these sections were incubated with TOM20 rabbit monoclonal antibody (dilution 1:400, Abcam) overnight incubation at $4^{\circ} \mathrm{C}$. The 
following day the samples were incubated with goat anti rabbit secondary antibody (Alexa fluor 488, Thermo Fisher, Rockford, IL, United States) for 2 hours at dark in room temperature. The nuclei were counterstained with Hoechst 33342 (Termofischer, Rockford,IL,United States) . Immunofluorescence staining of the isolated pancreatic acinar cells were performed freshly after the isolation procedure with the same conditions as stated above, (except two parameters ; cells were fixed in 2\% paraformaldehyde and dilution fo the primary antibody was 1:200) as stated above. Both ductal and acinar cell samples were mounted with Fluoromount and then analyzed using a Zeiss LSM 880 confocal laser scanning microscope (Carl Zeiss Technika Kft., Budaörs, Hungary). To quantify TOM20 positively stained area, 5-6 representative images from each group were taken by Zeiss LSM 880 Confocal Scannig Microscope (Carl Zeiss Technika Kft., Budaörs, Hungary). Image J software was used to convert images to gray scale (16 bit), threshold function was used to select the positively stained area. The fluorescence signal were calculated by the software (arbitary scale from 0-negative (white) to 255-maximal staining (black) ${ }^{[77]}$. Fluorescence intensity of the images were then normalized to the own total ductal or acinar area of the samples, which were measured in arbitary units. Fluorescence intensity was given in $\%$, normalized to the total ductal or acinar total area.

\section{V.4.8. In vivo measurements}

\section{V.4.8.1. Induction of acute pancreatitis}

AP was induced by caerulein $(\mathrm{CER}, 10 \times 50 \mu \mathrm{g} / \mathrm{kg})$ and $4 \%$ sodium taurocholate (TAU, $2 \mathrm{ml} / \mathrm{kg}, 4 \%)^{[49,78-80]}$. We also performed alcohol and fatty acid (intraperitonal injection of 1.75 $\mathrm{g} / \mathrm{kg}$ ethanol and $750 \mathrm{mg} / \mathrm{kg}$ palmitic acid, EtOH+FA) induced AP as described earlier [50, 81], however it is not part of this dissertation. All control groups received physiological saline in the same amount as the CER, EtOH+FA or the TAU solutions respectively.

\section{V.4.8.2. Oral gavage treatment of the mice}

Oral gavage treatment was performed using plastic feeding tubes $(20 \mathrm{ga} \times 38 \mathrm{~mm}$, Instech Laboratories, USA). NIM811 were solubilized in a vehicle which contained $8.3 \%$ polyoxyl 40 hydrogenated castor oil and 8.3\% ethanol [43]. Pre-treatment of the animals by NIM811 was performed and mice were gavaged orally once $1 \mathrm{~h}$ prior to the induction AP, concentrations of NIM811 were $10 \mathrm{mg} / \mathrm{kg}$ or $5 \mathrm{mg} / \mathrm{kg}$. Dosage of NIM811 was chosen according to a previous study in which NIM811 was effective against mitochondrial damage in liver transplantation ${ }^{[43]}$. 
Besides the pretreatment, NIM811 was used as a post-AP treatment as well. NIM811 was administered 12 hours after the induction of AP in the TAU or EtOH+FA induced experimental pancreatitis models. Concerning the CER induced AP, NIM811 was administered after the $3^{\text {rd }}$ injection of CER. The method for retrograde intraductal infusion of TAU has been described by Perides et al ${ }^{[80]}$. The surgery was performed on anesthetized mice (with ketamine-xylazine, dosage: $87.5 \mathrm{mg} / \mathrm{kg}$ ketamine- $12.5 \mathrm{mg} / \mathrm{kg}$ xylazine). At the end of the procedure the mice were placed on a heating pad for 40 minutes and received buprenorphine i.p. injection $(0.075 \mathrm{mg} / \mathrm{kg})$ at once to reduce their occurrent pain. Following these mice were replaced into their cages for 24hours. They had free access to food and water. 24 hours after the TAU or EtOH +FA induced AP the mice were euthanized by $200 \mathrm{mg} / \mathrm{kg}$ pentobarbital i.p. (Bimeda MTC, Cambridge, Canada). During the CER induced AP mice were euthanized with $200 \mathrm{mg} / \mathrm{kg}$ pentobarbital i.p. (Bimeda MTC, Cambridge, and Canada) 2 hours after the last injections of CER. Mice were exsanguinated through cardiac puncture and the pancreas were removed.

\section{V.4.9. Serum amylase measurements}

We collected blood from the mice by cardiac puncture, blood was immediately placed on ice, then centrifuged with $2500 \mathrm{RCF}$ for 15 mins at $4^{\circ} \mathrm{C}$. Blood serum was collected from the pellet and stored at $-20^{\circ} \mathrm{C}$ until use. Pancreas samples were placed into $8 \%$ neutral formaldehyde solution and stored at $-4^{\circ} \mathrm{C}$ until the hematoxylin -eosin staining was performed. A colorimetric kit was used to measure serum amylase activity (Diagnosticum, Budapest, Hungary). Absorbance of the samples were detected at $405 \mathrm{~nm}$ with the use of FLUOstar OPTIMA (BMG Labtech, Budapest, Hungary) microplate reader.

\section{V.4.10. Histological analysis}

Formaldehyde-fixed pancreas samples were embedded in paraffin, then were cut into sections $(3 \mu \mathrm{m})$ and hematoxylin-eosin staining were performed by using a standard laboratory method. To quantify histological differences a semiquantitative scoring system was used as Kui et al described previously ${ }^{[82]}$.

\section{V.5.Materials and methods used in publication No.2.}

\section{V.5.1. Animals}

CFTR knock out (KO) (background FVB/N) mice were kindly provided by Dr. Ursula Seidler (Hannover Medical School,Hannover,Germany). AQP1 KO (background CD4) ( mice were 
supplied by Dr. Alan Verkman (University of Carolina, CA,Unites States) and Dr. Alastair Poole (University of Bristol,United Kingdom). Animals were kept in a room maintained at $23 \pm 1{ }^{\circ} \mathrm{C}$ on a $12 \mathrm{~h}$ light-dark cycle with food and water available ad libitum. CFTR specific laboratory chow were administered to CFTR KO animals. Experiments were performed on 1216 weeks old mice (sex ratio 1:1) from litter-matched groups.

\section{V.5.2. Immunfluorescent stainings and detection of AQP1 and CFTR channels in mouse pancreas}

$7 \mu \mathrm{m}$ thick cryosections from WT, AQP1, and CFTR KO mice pancreas were fixed in 2\% paraformaldehyde. Permeabilisation of the slices occured in $10 \%$ Tween 20-sodium citrate, they were blocked with 5\% goat serum. Immunofluorescent double staining for AQP1 mouse monoclonal antibody (1:500 dilutions; Thermo Fisher, Rockford, IL, United States) and CFTR rabbit polyclonal antibody (1:100 dilutions; Alomone Labs, Jerusalem, Israel) were performed by overnight incubation at $4 \circ \mathrm{C}$. After the washing periods, slices were incubated with secondary antibodies goat-anti-mouse (Alexa fluor 488, Thermo Fisher, Rockford, IL, United States) and goat-anti-rabbit (Alexa fluor 568, Thermo Fisher, Rockford, IL, United States) for 120 minutes at room temperature in the dark. Nuclei staining were performed with the use of DAPI fluorescent dye. Results of the immunostaining were then analyzed using a Zeiss LSM 880 confocal laser scanning microscope (Carl Zeiss Technika Kft., 10-12 representative pictures were taken from the mice (WT, AQP1 KO and CFTR KO) pancreas sections. Digital images were taken at 40x magnification. Samples were excited at 405 (DAPI), 488 (Alexa fluor 488) and 568 (Alexa fluor 568) $\mathrm{nm}$ and emissions were collected at 453, 516, and $603 \mathrm{~nm}$, respectively. Following this step, Image $\mathbf{J}$ software were used and images were then converted to gray scale (16-bit) and thresholded to select the positively stained area, using the ImageJ software. The fluorescence intensity (mice pancreas) were arbitrary scaled from 0 to 255, where 0 were the negative staining (white pixels) and 255 were the maximal staining (black pixels). In the mice pancreas slices, the ductal area was selected and the total fluorescence intensity was calculated and summarized which was then normalized to the ductal area $(\mu \mathrm{m} 2)$ and expressed in arbitrary units $/ \mu \mathrm{m} 2$.[77]

\section{V.6. Methods used in Publication No.3.}

\section{V.6.1. Animals}

We performed our experiments on female FVB/N mice (Charles Rivers Laboratories, Wilmington, MA, USA). The mice were housed in standard plastic cages which were kept in 
room temperature of $23-24{ }^{\circ} \mathrm{C}$ and kept in $12 \mathrm{~h}$ light-dark cycle in the animal facility of First Department of Medicine, University of Szeged. Animals could free access of water and standard laboratory chow (Biofarm, Zagyvaszántó, Hungary).

\section{V.6.2. Development of the new model of MA in mice}

To develop a mouse model of chronic MA, the mice were randomly divided into the following 4 groups for a 12-day treatment:

- ammonium chloride $\left(\mathrm{NH}_{4} \mathrm{Cl}^{-}\right)$administration with drinking water $(8.2 \pm 0.5$ $\mathrm{ml} /$ day/mouse) as described earlier ${ }^{[83,84]}$

- intraperitoneal (i.p.) injections of $\mathrm{NH}_{4} \mathrm{Cl}^{-}(0.5 \mathrm{ml}, 0.28 \mathrm{M})$ on days 1 and 6;

- administration of $\mathrm{NH}_{4} \mathrm{Cl}^{-}$with drinking water (as in group 1) and i.p. injections (as in group 2);

- and controls, receiving $\mathrm{NH}_{4} \mathrm{Cl}^{-}$free tap water and 2 i.p. injections of saline on days 1 and 6.

\section{V.6.3. Induction of AP}

Severe AP (SAP) was induced by caerulein (CER,10x50 $\mu \mathrm{g} / \mathrm{kg})$, CER was administered i.p. ${ }^{[78]}$. Mild AP was induced by alcohol and fatty acid (i.p. of $1.75 \mathrm{~g} / \mathrm{kg}$ ethanol and $750 \mathrm{mg} / \mathrm{kg}$ palmitic acid , EtOH+FA) as described previously ${ }^{[50,81]}$. During the experimental model of MA, MAP and SAP were induced on day 12 of the acidifying treatment.

\section{V.6.4. Measurement and histological analysis}

Laboratory parameters from blood serum and urine were performed by standard methods at the Institute of Laboratory Medicine, University of Szeged. Serum amylase measurement and histological analysis were performed as described in the previous chapters respectively. For blood gas $\mathrm{pH}$ measurements, samples of arterial blood $(170 \mu \mathrm{l})$ were collected from the mice in heparin and lithium treated and sealed plastic capillaries. Analysis of the arterial blood was performed by blood gas analyser (Cobas 221, Roche Ltd., Basel,Switzerland) within 1 minute after the blood collection (at room temperature $22^{\circ} \mathrm{C}$ ). 


\section{RESULTS}

\section{VI.1. Results of publication No.1.}

\section{VI.1.1. Genetic inhibition of mPTP protects mitochondrial homeostasis and cell function} evoked by pancreatitis-inducing factors in PDEC

As a beginning, we studied the effects of the most relevant pancreatitis-inducing factors on mitochondria in primary intact ducts isolated from $\mathrm{Ppif}^{-/-}$and WT mice. Experiments performed with TMRM and TOM20 revealed that genetic inhibition of mPTP decreased both the loss of $\Delta \psi$ (Fig. 1A) and mitochondrial mass (Fig. 1B) caused by BA or co-administration of EtOH+FA. Genetic inhibition of mPTP also decreased the extent of necrosis and apoptosis during the administration of $\mathrm{BA}$ or $\mathrm{EtOH}+\mathrm{FA}$ (Fig. 1C), suggesting that genetic inhibition of Cyp D has a protective effect on PDEC. Next, we investigated how the genetically preserved mitochondrial function affects the cellular function of PDEC (Fig. 1D). We used the $\mathrm{NH}_{4} \mathrm{Cl}$ pulse technique, which is uniquely suited to characterizing both $\mathrm{HCO}_{3}{ }^{-}$influx and efflux mechanisms. Our experiments demonstrated that the inhibitory effects of BA and EtOH+FA on $\mathrm{Cl} / \mathrm{HCO}_{3}{ }^{-}$exchangers $\left(\mathrm{HCO}_{3}{ }^{-}\right.$efflux $)$and on $\mathrm{Na}^{+} / \mathrm{HCO}_{3}{ }^{-}$co-transporters $\left(\mathrm{HCO}_{3}{ }^{-}\right.$influx $)$are totally blocked in Ppif ${ }^{--}$vs WT mice, suggesting that inhibition of mPTP can preserve ductal function and thus has therapeutic benefits (Fig. 1D-F). 

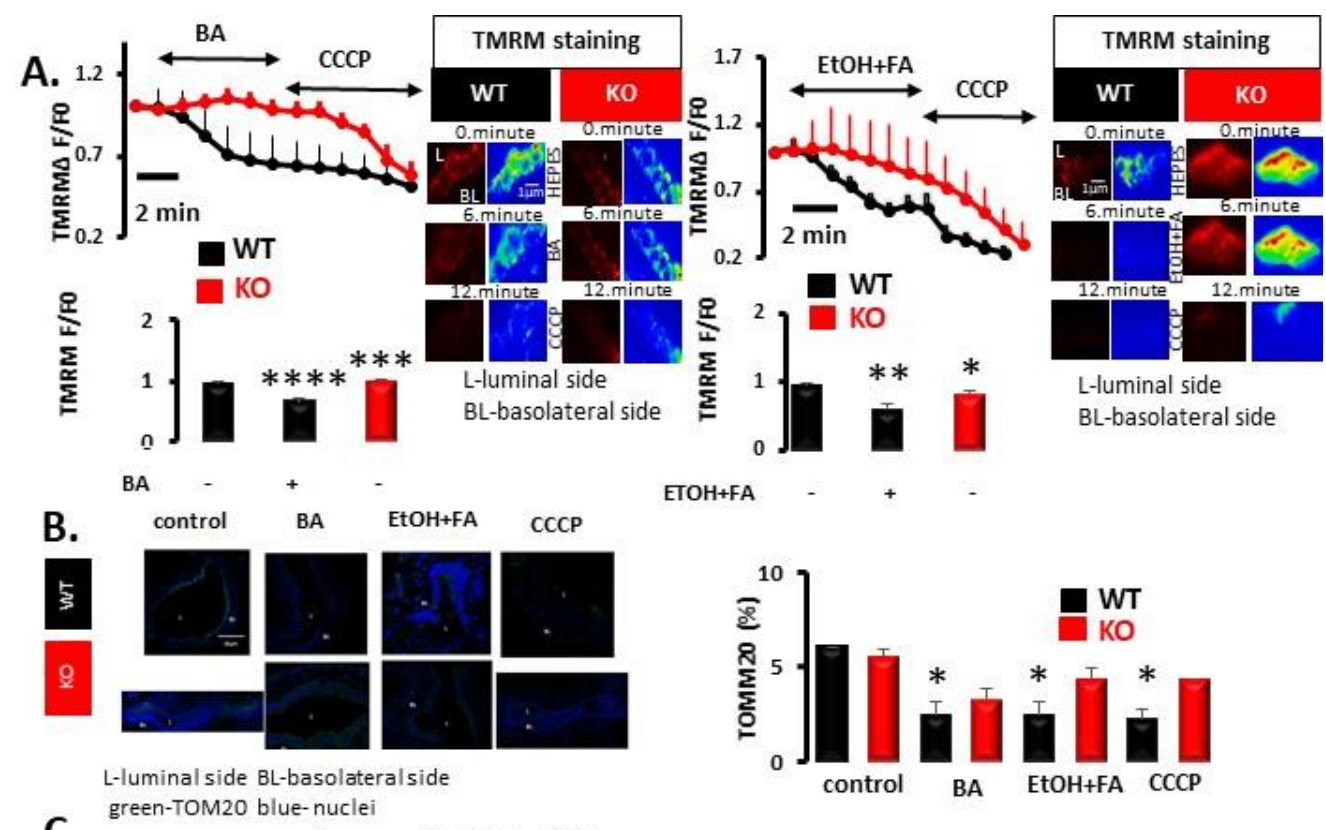

$\mathrm{ETOH}+\mathrm{FA}$

C.
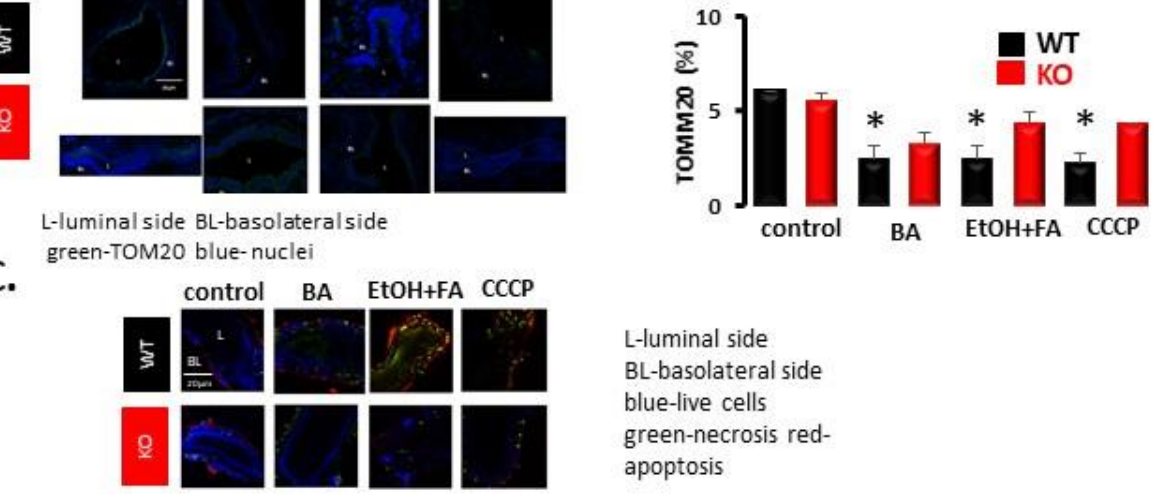

L-luminal side

BL-basolateral side

blue-live cells

green-necrosis red-

apoptosis
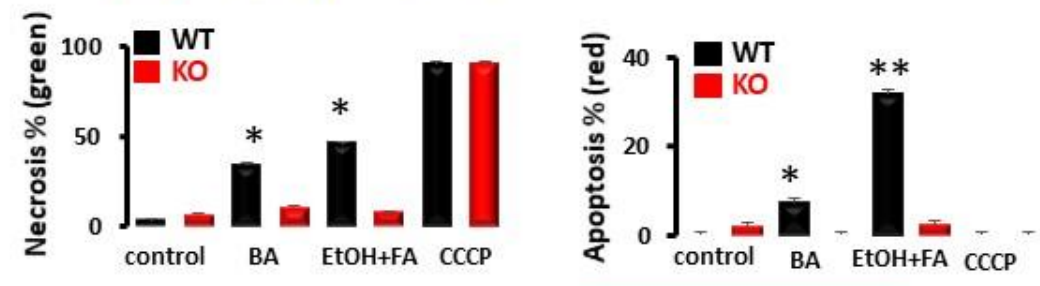

D.
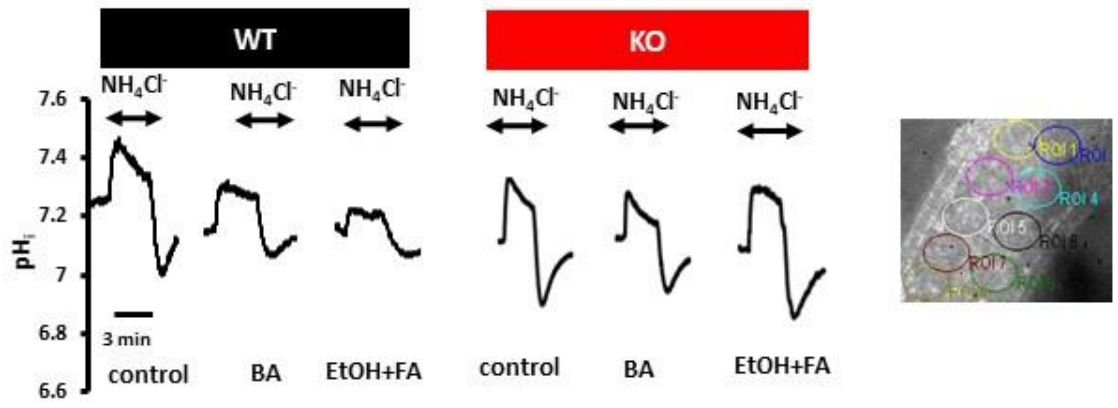

E.
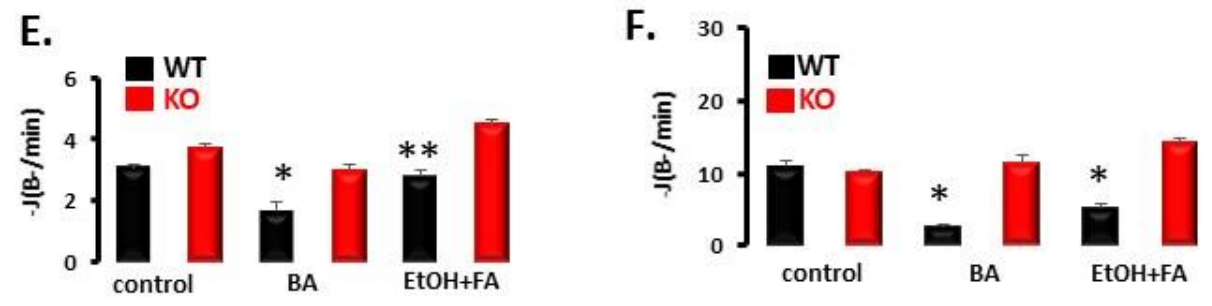

Figure1. Genetic inhibition of Cyp D reduces the severity of bile acid or ethanol and fatty acid induced damage in PDEC

Genetic inhibition of mPTP significantly reduces the mitochondrial membrane potencial loss compared to WT controls during the administration of BA or EtOH+FA ( WT control vs WT 
BA ***p $<0.001$,WT BA vs Cyp D KO BA **p $<0.002$, WT control vs Cyp D KO BA $\mathrm{p}=07.12$, WT control vs. WT EtOH+FA $\mathrm{p}<0.01$, WT EtOH+FA vs KO ETOH+FA * p $<0.05$, WT control vs Cyp D KO EtOH+FA p=0.145) $n=4-6$ experiments/group, data means \pm SEM. Results from the immunostainings revealed a significant decrease of the TOM20 stainings in BA; $\mathrm{EtOH}+\mathrm{PA}$ or CCCP treated WT ducts, results were compared to Cyp D KO stainings . (Fig1.B) $\left({ }^{*} \mathrm{p}<0.05\right)$. Genetic inhibition of mPTP also decreased the necrosis and apoptosis levels during bile acid; ethanol or fatty acid or CCCP treatment (Fig1.C). $\quad(* \mathrm{p}<0.05)$ Representative traces from the pancreatic ductal $\mathrm{HCO}_{3}$ - secretion measurements (Fig.1.D) Our data revealed that recovery from the alkalosis grades were significantly lower due to BA or ETOH+FA administration ( $\left.{ }^{*} \mathrm{p}<0.05\right)$ compared to the results from Cyp D KO ducts (Fig1.E). Recovery from the acidosis grades were significantly lower in the WT ducts due to the treatment with BA or EtOH and FA $\left({ }^{*} p<0.05\right)$, while in Cyp D KO ducts these grades were significantly higher $\left({ }^{*} \mathrm{p}<0.05\right)$. $\mathrm{n}=5-7$ experiments/group, data means \pm SEM.

\section{VI.1.2. Pharmacological inhibition of mPTP by CyA effectively prevents mitochondrial damage evoked by pancreatitis-inducing factors in PDEC.}

$\mathrm{BA}$ and $\mathrm{EtOH}+\mathrm{FA}$ significantly decreased the $\psi$ of PDEC (Fig. 2A). Importantly, $2 \mu \mathrm{M}$ CYA effectively blocked the toxic effects of the BA- and EtOH+FA-preserving function of mitochondria during the presence of pancreatitis-inducing factors. As regards the quantity of mitochondria, CYA effectively inhibited loss, as we could see during the genetic inhibition of mPTP (Fig. 2B). $2 \mu \mathrm{M}$ CYA decreased the extent of necrosis and apoptosis during the administration of BA or EtOH+FA in PDEC (Fig. 2C). Finally, we provided strong evidence of the beneficial effects of CYA on mPTP noted above, mitochondrial mass and cell death, resulting in preserved $\mathrm{HCO}_{3}{ }^{-}$efflux and influx mechanisms during BA or EtOH-FA administration (Fig. 2D-F). 

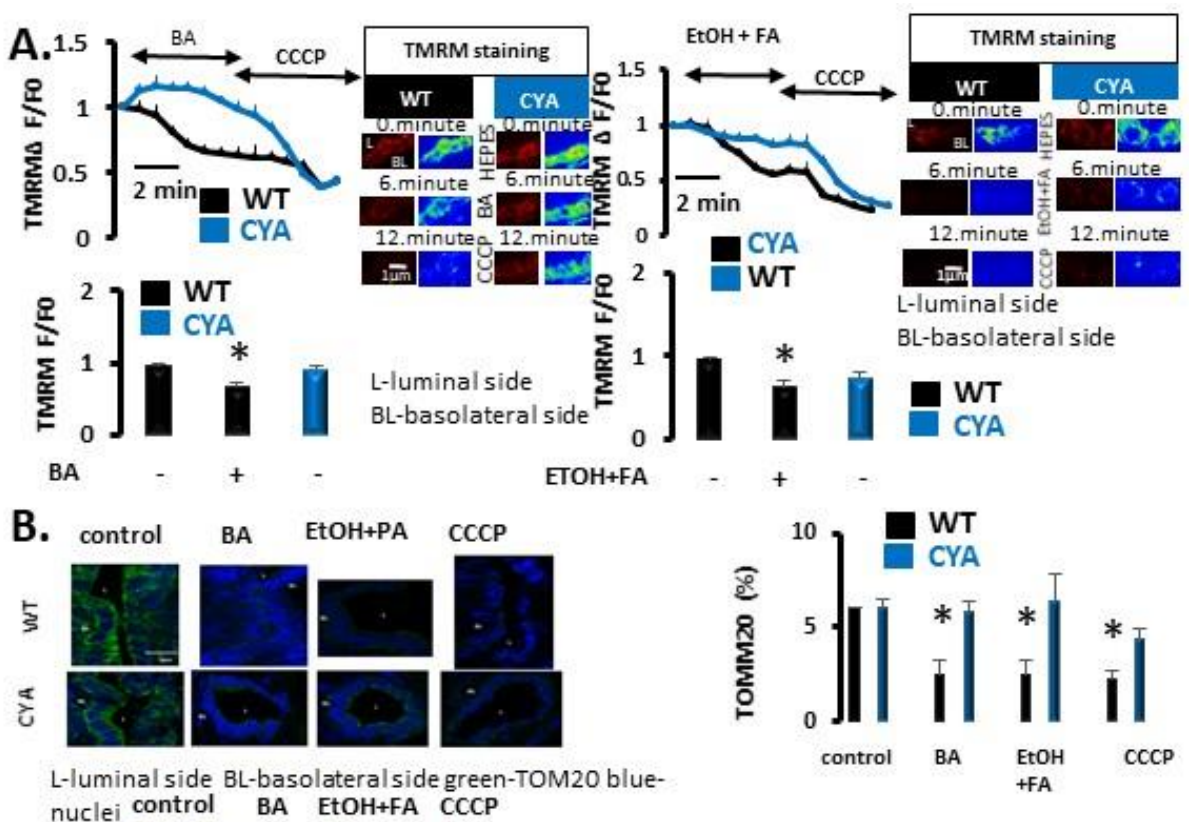

C.
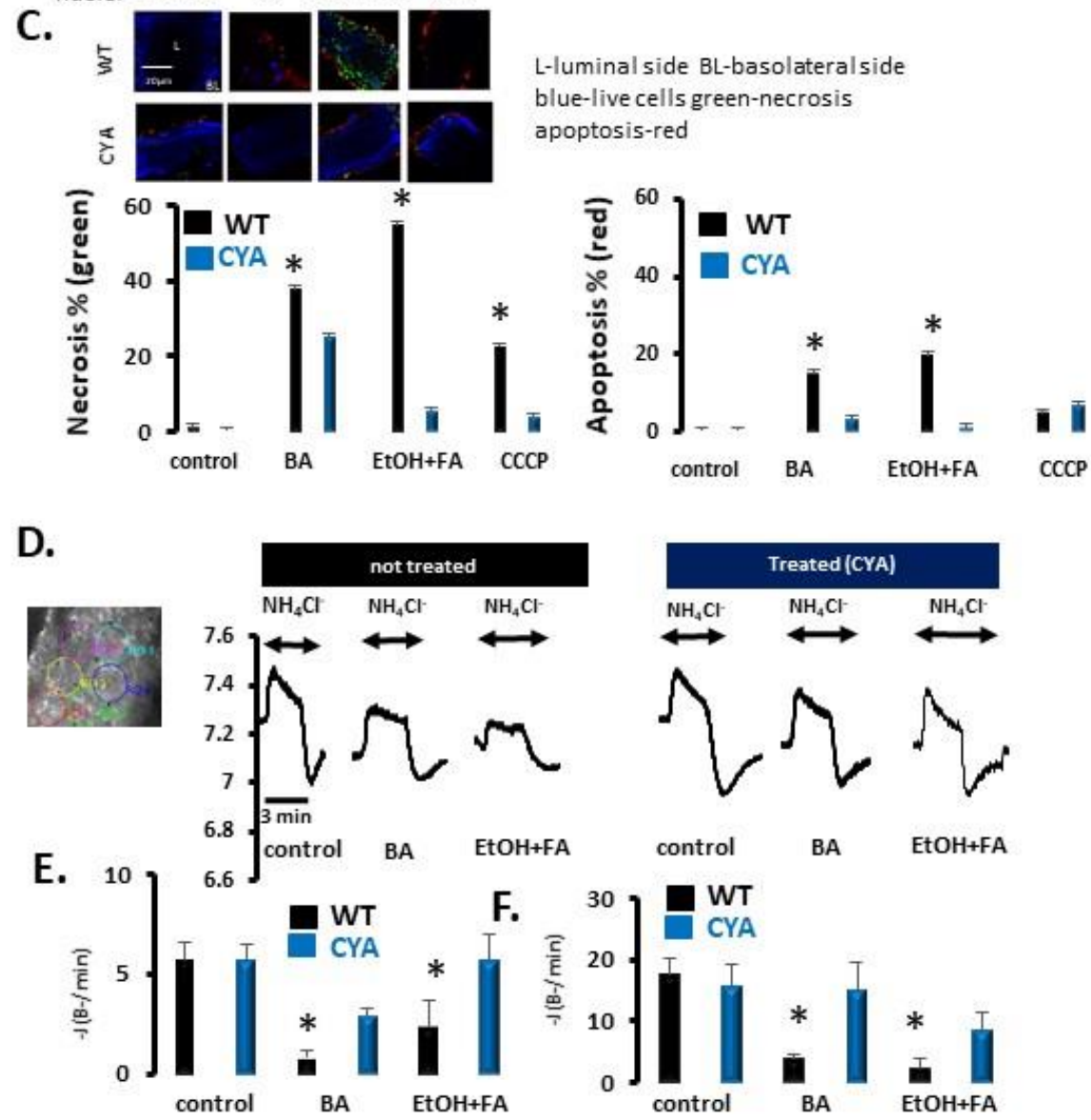

Figure2. CYA reduces the severity of bile acid or ethanol and fatty acid induced pancreatic ductal damage Treatment of the ducts with CYA reduced the drop of mitochondrial membrane potencial loss which accured due to the BA or ETOH+FA treatment. (WT vs. CYA) (Fig.2.A). In WT ducts $\mathrm{BA}$ or $\mathrm{ETOH}+\mathrm{FA}$ treatment resulted in significantly reduced mitochondrial membrane potencial (WT control vs WT BA *p<0.05, WT control vs WT EtOH + FA p $<0.05$ ), 
while between WT control groups compared to CYA treated BA or EtOH+FA there were no significant decrease. TOM20 levels were significantly reduced in BA; ETOH+FA or CCCP control (not CYA treated) ducts, while in the CYA treated groups the percentage of TOM20 stained area were significantly higher (Fig2.B) $* p<0.05$. Between the control groups (WT control or only CYA treated samples) we found no significant alterations in the stainings. Necrosis levels were intensively elevated in BA or EtOH treated groups in WT ducts but not in CYA treated groups (Fig.2.C). Apoptosis levels were significantly higher as well in the not CYA treated groups compared to the CYA treated groups (Fig2. C). Measurements of $\mathrm{HCO}_{3}{ }^{-}$ secretion levels revealed a significant difference in WT and CYA treated ducts during the administration of BA $\left(p<0.05\right.$ WT BA vs CYA BA) or EtOH+FA $\left({ }^{*} p<0.05\right)$. In WT ducts the levels of base flux (-J(B-/min) grades were significantly decreased (Fig2.E,F) due to BA (WT vs WT BA p<0.05) or ETOH+FA (WT vs WT EtOH+PA p<0.05) treatment (Fig2 E,F). Recovery from alkalosis (Figure 2. E) and recovery from acidosis values are presented in base flux ((-J(B-/min) grades respectively, with \pm SEM. Comparison within CYA treated groups revealed no significant difference (CYA control vs CYA BA p $=0.644)$.

\section{VI.1.3. NIM811 treatment protects mitochondrial function and bicarbonate transport mechanisms in PDEC}

We investigated the effects of the novel CYA derivative NIM811 on mitochondrial function and of bicarbonate secretion on isolated pancreatic ducts. According to our data, NIM811 reduces the $\mathrm{BA}$ - or $\mathrm{EtOH}+\mathrm{FA}$-induced damage to mitochondrial function and morphology in isolated pancreatic ducts (Fig. 3A-B). NIM811 alone has no toxic effects on PDEC. Furthermore, it can strongly decrease BA- or EtOH-FA-evoked necrosis and apoptosis (Fig. $3 \mathrm{C}$ ). $\mathrm{NH}_{4} \mathrm{Cl}^{-}$experiments revealed that the inhibitory effects of $\mathrm{BA}$ and $\mathrm{EtOH}+\mathrm{FA}$ on $\mathrm{Cl} / \mathrm{HCO}_{3}{ }^{-}$ exchangers $\left(\mathrm{HCO}_{3}{ }^{-}\right.$efflux $)$and on $\mathrm{Na}^{+} / \mathrm{HCO}_{3}{ }^{-}$co-transporters $\left(\mathrm{HCO}_{3}{ }^{-}\right.$influx $)$were significantly reduced in the NIM811-treated groups compared to the controls, showing a protective effect of NIM811 on PDEC (Fig. 3D). 


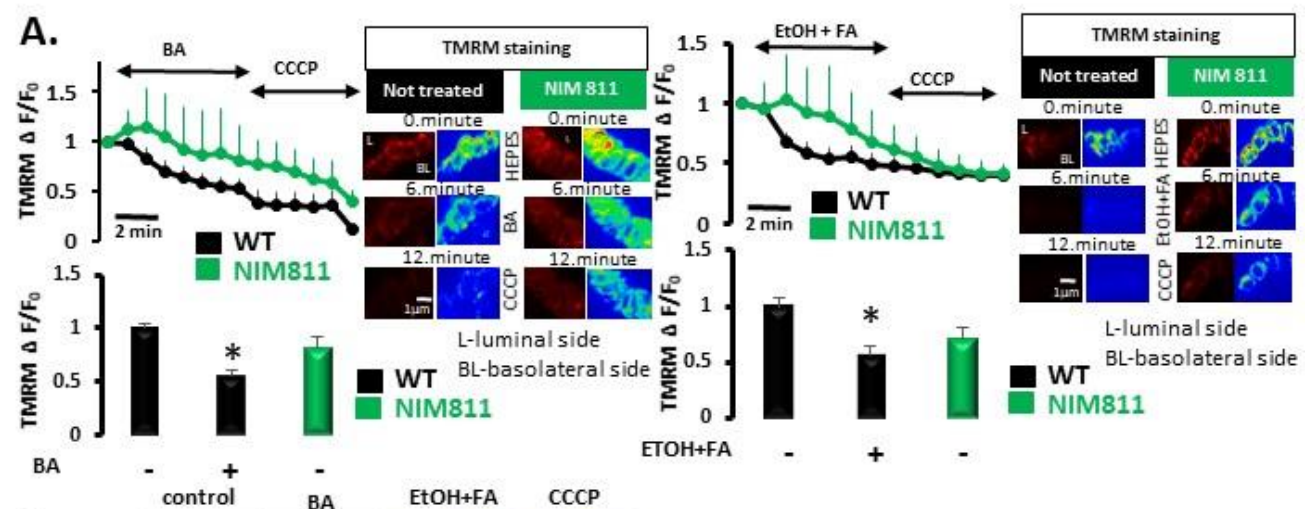

B.
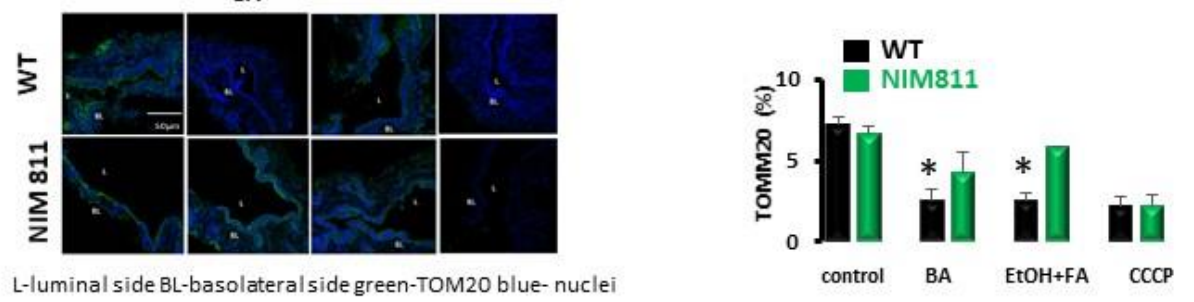

C. control BA EtOH+FA CCCP

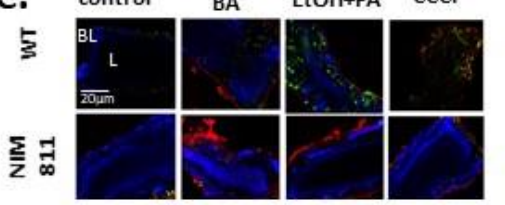

L-luminal side, BL-basolateral side, blue-

live cells, green-necrosis, apoptosis-red
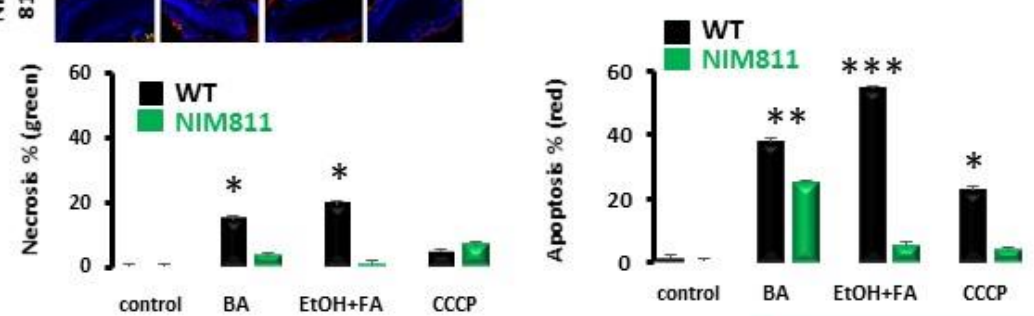

D.
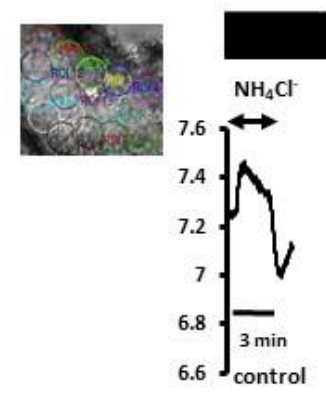

Not treated

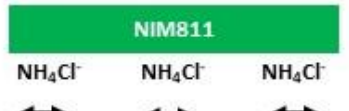

$\stackrel{\mathrm{NH}_{4} \mathrm{Cr}}{\leftrightarrow} \stackrel{\mathrm{NH}_{4} \mathrm{Cl}}{\leftrightarrow}$
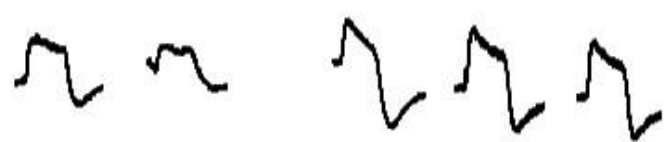

BA EtOH+FA control BA EtOH+FA

E.

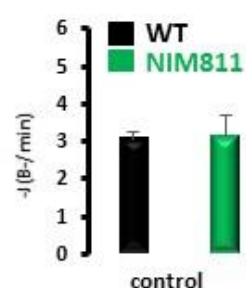

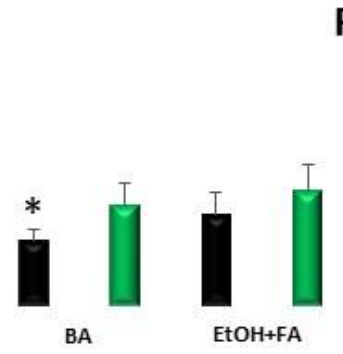

F.

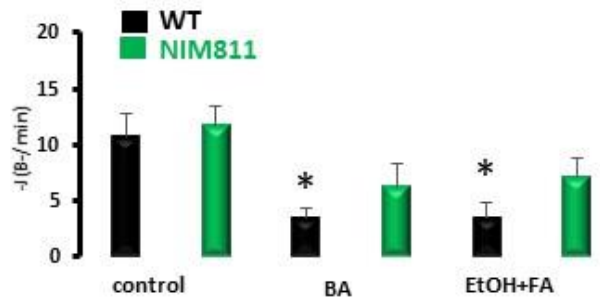

Figure3. NIM811 protects mitochondrial and cell function in PDEC. NIM811 treated ducts revealed a significantly consolidated loss of mitochondrial membrane potencial during the BA 
(WT BA vs NIM811 BA *p<0.05) or ETOH+FA (WT ETOH+FA vs NIM811 ETOH+FA $\left.{ }^{*} \mathrm{p}<0.05\right)$ treatment (Fig.3A) . In NIM811 treated ducts the percentage of fluorescence intensity were significantly higher compared to not NIM811 treated ducts during BA or ETOH+FA administration. In CCCP treated ducts we found no significant difference in the amount of TOM20 stainings in the aspect of NIM811 treated or not treated groups. NIM811 itself did not alter the value of TOM20 stainings compared to the WT control samples (Fig.3B). NIM811 decreased the numbers of apoptotic and necrotic cells during bile acid or ethanol and fatty acid treatment (Fig.3C) (WT BA vs NIM811 BA *p<0.05, WT EtOH+FA vs NIM811*p<0.05). While during the administration of CCCP the apoptosis and necrosis grades were not significantly different in the comparative groups (Fig.3.C). NIM811 treatment did not decreased the $\mathrm{HCO}_{3}{ }^{-}$secretion grades (control, Fig.3 D,E,F), while during the adminsitration of BA or $\mathrm{ETOH}+\mathrm{FA}$ treatment it had a protective effect against the reduction of $\mathrm{HCO}_{3}{ }^{-}$secretory levels (Fig.3E/F) (WT BA vs NIM811 BA *p<0.05, WT EtOH+FA vs NIM811 EtOH+FA *p<0.05). In the aspect of recovery levels from alkali load during EtOH and FA treatment, the difference were not sigfnificant in WT EtOH+FA compared to the NIM811 and EtOH+FA treated groups (Fig.3E).

\section{VI.1.4. Pharmachological inhibition of $\mathrm{mPTP}$ have no effects on pancreatic ductal fluid secretion}

Both in vivo and in vitro measurements revealed that NIM811 or CyA treatment can not prevent BA or EtOH+FA induced fluid secretiory damage in isolated ducts (Fig.4 A-D, E-F). 


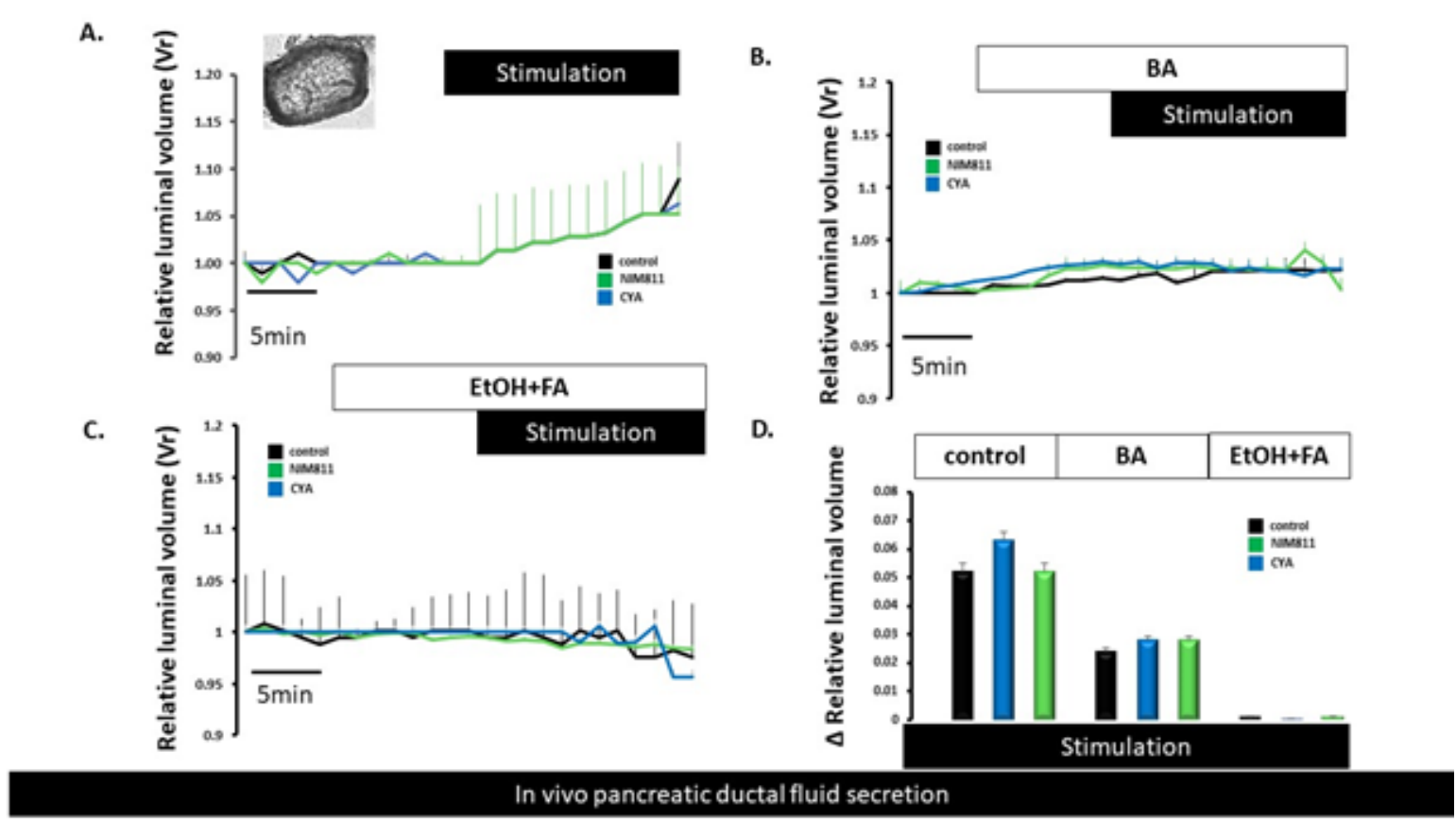

E.

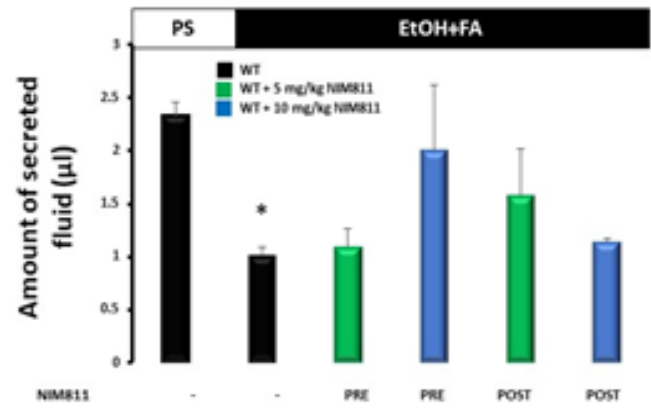

F.

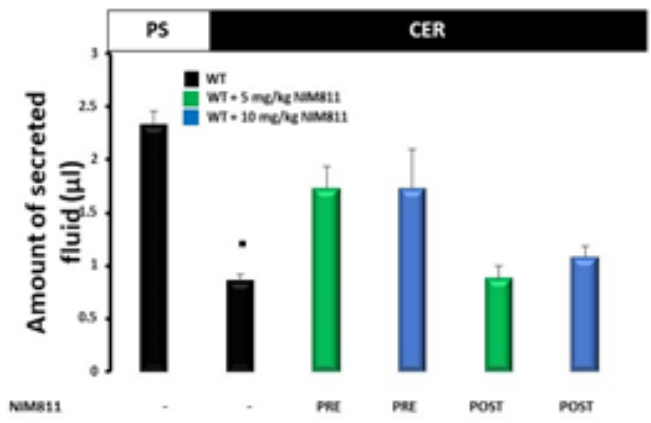

Figure 4. Pancreatic ductal fluid secretion is not altered by NIM811 or CYA treatment . In vitro fluid secretion was stimulated by $5 \mu \mathrm{M}$ forskolin and $100 \mu \mathrm{M}$ IBMX (stimulation). BA or $\mathrm{EtOH}+\mathrm{PA}$ treatment inhibited the luminal swelling (Fig.4.B-C). Figure 4D represents the relative luminal volume changes during forskolin and IBMX stimulation (Figure4.D). Means \pm SEM. $\mathrm{n}=5$-10 ducts/group. In vivo fluid secretion measurements were performed after the induction of CER or EtOH+FA induced AP (Fig.4.E-F.). These experiments confirmed that pancreatic ductal fluid secretion is not affected by NIM811 or CyA. (Fig.4.E-F). ${ }^{*} \mathrm{p}<0.05$ WT PS vs. WT EtOH+FA, $-p<0.05$ WT PS vs. WT CER n=4-7 animal/group

\section{VI.1.5. NIM811 treatment protects mitochondrial function in acinar cells}

In vitro measurements of freshly isolated pancreatic acinar cells showed that NIM811 treatment decreased the BA- and EtOH-FA-induced loss of $\psi$ as effectively as we have seen in PDEC (Fig. 4A). However, results obtained from TOM20 staining suggest that NIM811 has no effect 
on mitochondrial mass in acinar cells (Fig. 5B). Microfluorometric measurements demonstrated that NIM811 alone has no toxic effects on acinar cells and has no effect on BA- or EtOH-FAinduced apoptosis, but is protective against BA- or EtOH-FA-induced necrosis (Fig. 5C).
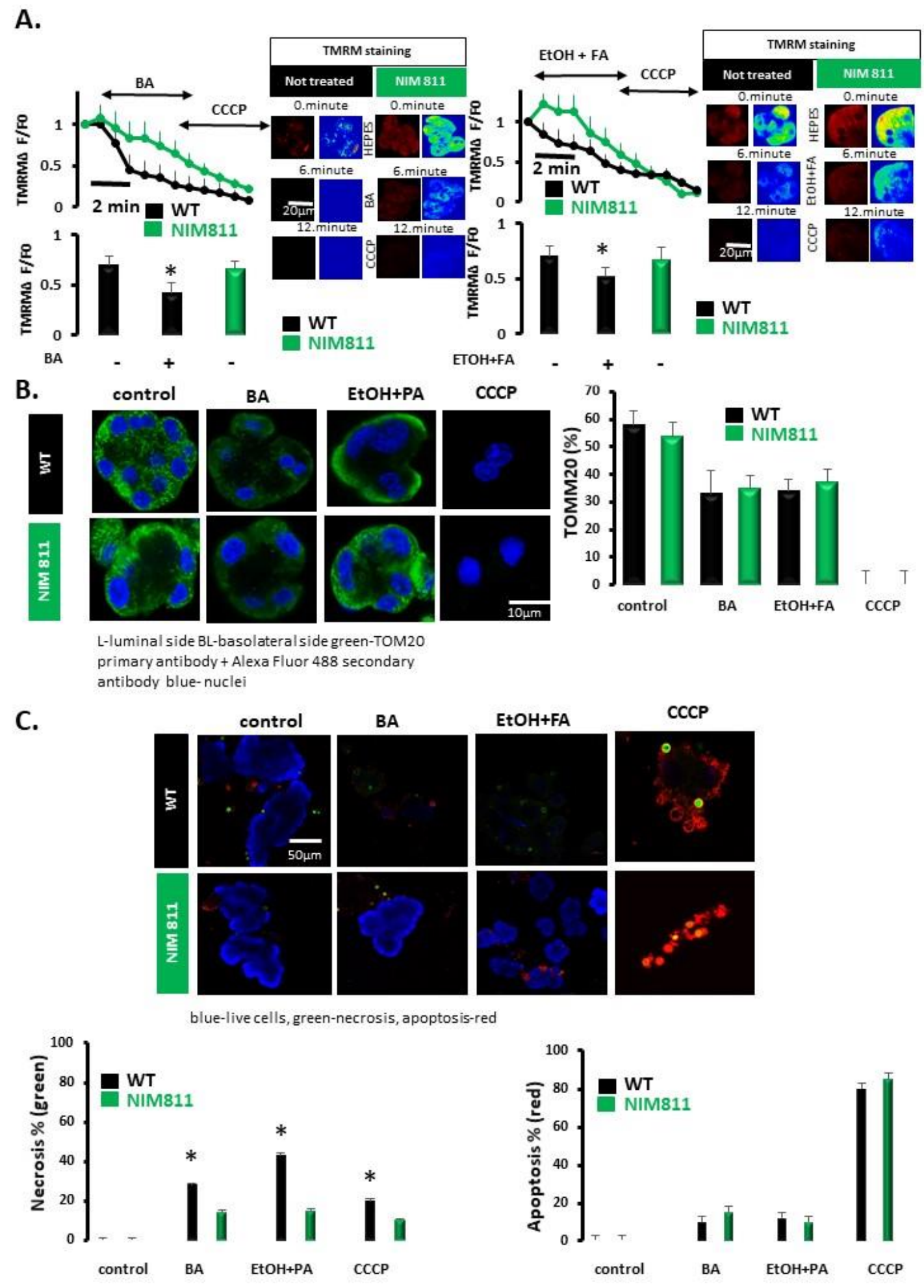

Figure5. NIM811 treatment protects mitochondrial function in pancreatic acinar cells 
Mitochondrial membrane potencial measurements revealed a significant difference between WT not NIM811 treated and the NIM811 treated acinar cell response due to bile acid or ethanol and fatty acid treatment (Fig.5A) (WT BA vs NIM811 BA *p<0.05; WT EtOH+FA vs NIm811 $\mathrm{ETOH}+\mathrm{FA} * \mathrm{p}<0.05)$. Significant difference was detected between the NIM811 treated acinar cells and the groups which were not treated with NIM811 (Fig.5A) during BA or ETOH+FA treatment. Mitochondrial protein TOM20 levels did not show difference in the NIM811 treated or not treated groups after BA, ETOH+FA or CCCP treatment (Fig.5B) (p>0.05). In necrosis levels we found significant difference between NIM811 treated and not treated groups in BA or ETOH+FA (Fig.5C) $(* p<0.05)$. However, in CCCP treated groups we found no difference (Fig.5C). Apoptosis levels were not altered significantly by NIM811 during BA or ETOH+FA treatment.

\section{VI.1.6. NIM811 reduces the severity of AP in mice}

Firstly, we confirmed that per os administration of either 5 or $10 \mathrm{mg} / \mathrm{kg}$ NIM811 alone has no toxic offect the pancreas (Fig 6.)
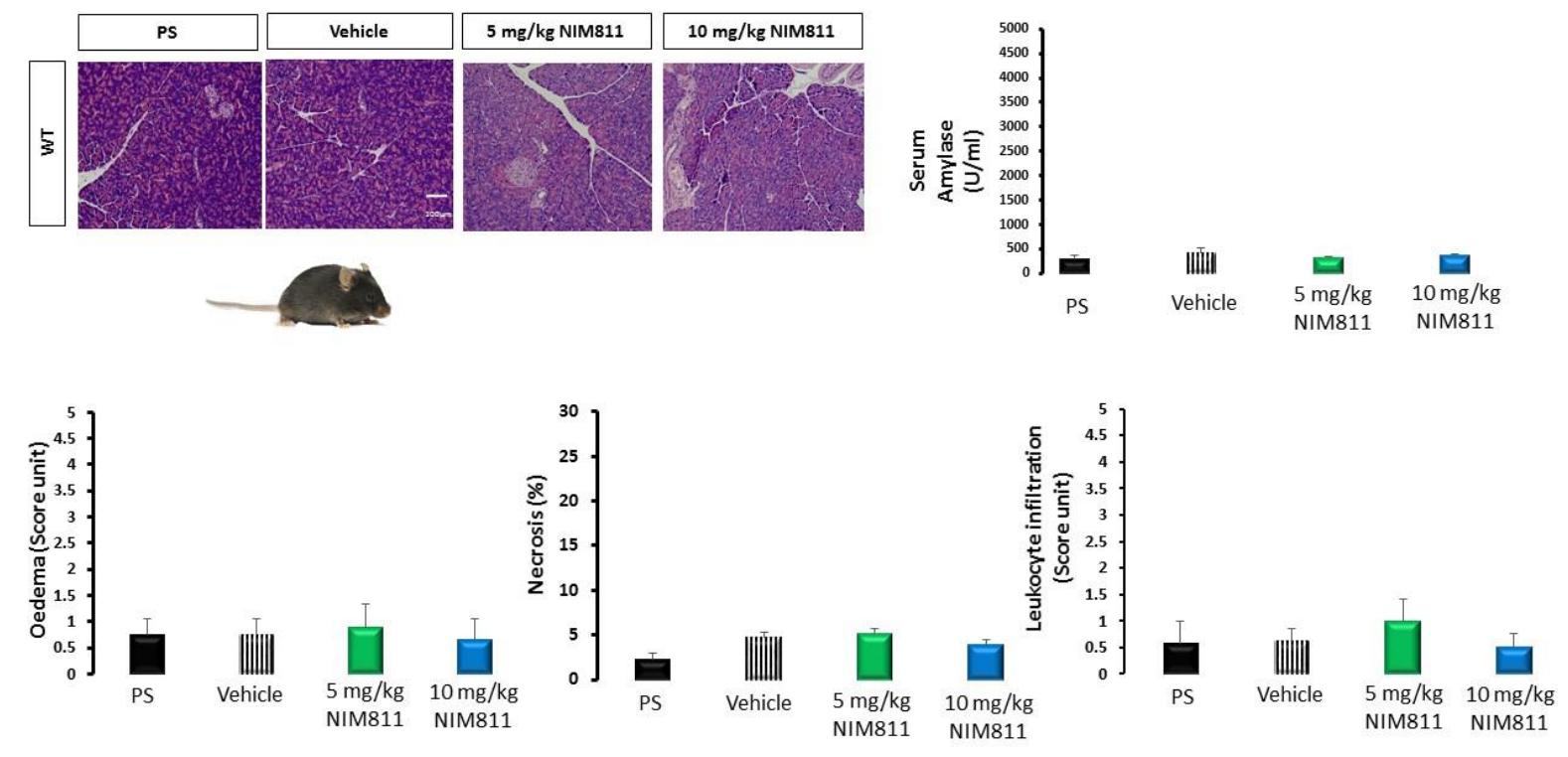

Figure6. NIM811 itself does not induce pancreatic damage . No significant difference was found between the NIM811-treated - (8.3\% Polyoxyl 40 hydrogenated castor oil, $8.3 \% \mathrm{EtOH})$ vs. the control groups. $n=4-5$ animal/group

Secondly, we tested the compound in two different experimental AP models, the caerulein (CER) and the taurocholic (TAU)-induced ones (Niederau et al,1985; Huang et al, 2014) . Importantly, both pretreatment 5 or $10 \mathrm{mg} / \mathrm{kg}$ NIM811 significantly reduced the elevation of 
serum amlylase activity, as well as pancreatic oedema, necrosis and leukoctye infiltration in experimental AP models (Figs. 7-8). In our study we also confirmed, that post treatment of $5 \mathrm{mg} / \mathrm{kg}$ or $10 \mathrm{mg} / \mathrm{kg}$ NIM811 has protective effects against pancreatic damage (Figs. 7-8.).
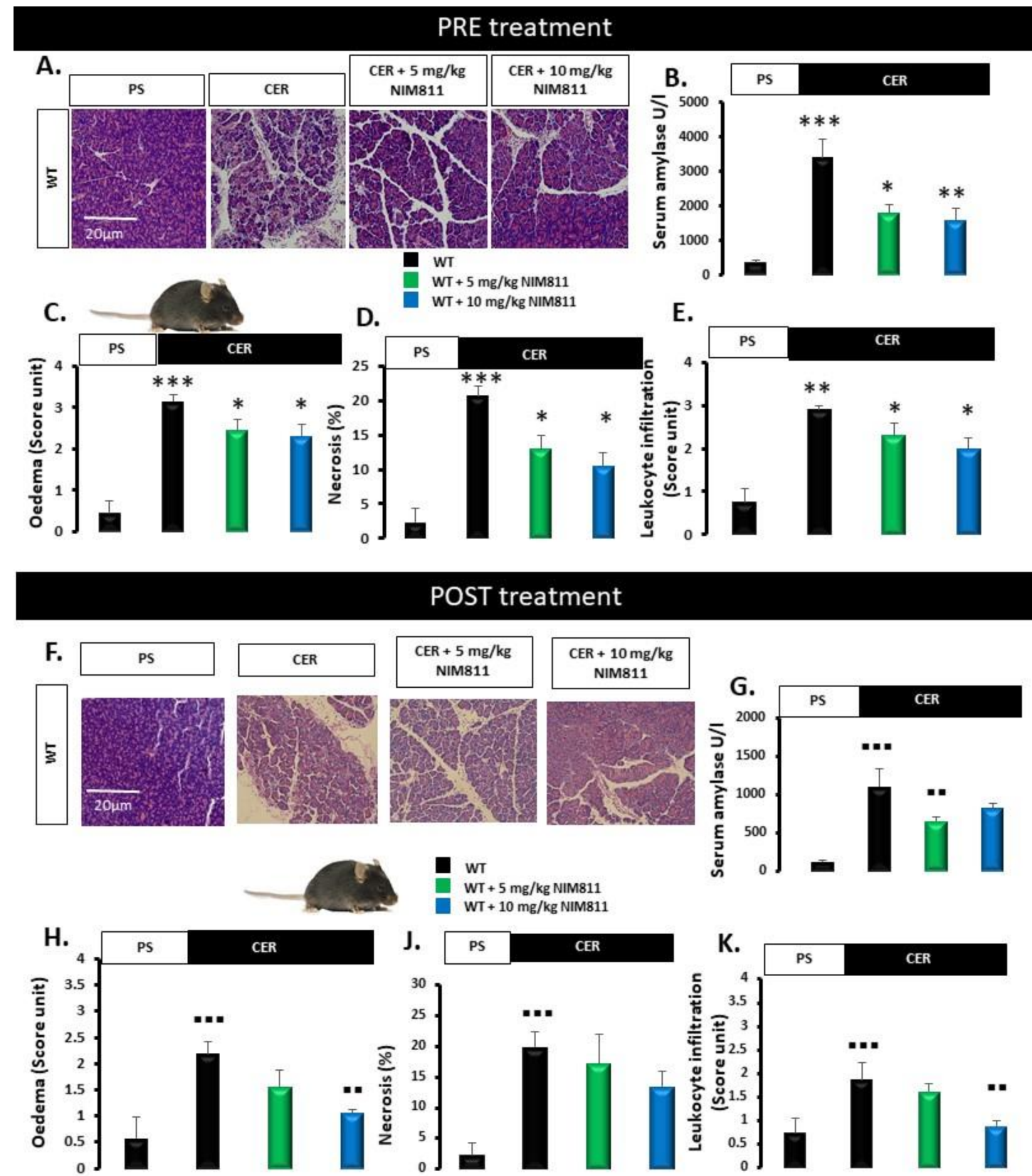

\section{Figure 7. NIM811 reduces the severity of CER induced AP}

Serum amylase levels were increased in the CER treated groups and NIM811 treatment resulted in a reduced serum amylase levels during CER induced AP compared to WT CER group (Fig. 7B ***p $<0.01$ WT PS vs WT CER, ${ }^{* *} \mathrm{p}<0.02$ WT CER vs pre10mg/kg NIM811 CER, ${ }^{*} \mathrm{p}<0.05$ 
WT CER vs pre $5 \mathrm{mg} / \mathrm{kg}$ NIM811 CER, p=0.717 CER + pre $5 \mathrm{mg} / \mathrm{kg}$ NIM811 vs CER + pre 10mg/kg NIM811 ). In CER induced pancreatitis both $5 \mathrm{mg} / \mathrm{bwkg}$ NIM811 (Fig.7 A-F, p $<0.05$ WT CER vs. pre 5mg/bwkg NIM811 CER) and pre 10 mg/bwkg NIM811 (Fig.7 A-F, p $<0.05$ WT CER vs. Pre 10mg/bwkg NIM811 CER)) treatment reduced the CER-induced damage. Post $5 \mathrm{mg} / \mathrm{kg}$ NIM811 treatment significantly reduced serum amylase levels compared to WT CER $-" p<0.05, \quad \cdots p<0.001$ WT PS vs WT CER (Fig.7G-E). Post insult administration of $10 \mathrm{mg} / \mathrm{kg}$ NIM811 significantly reduced oedema and leukocyte infiltration levels compared to WT CER treated groups $\cdot-\mathrm{p}<0.05($ Fig. $7 \mathrm{H}), \mathrm{n}=8-10$ animals per group, data means \pm SEM).

\section{PRE treatment}

A.
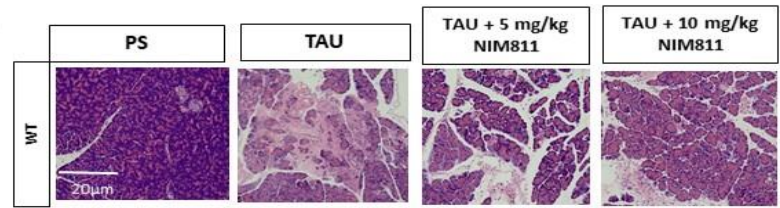

P.
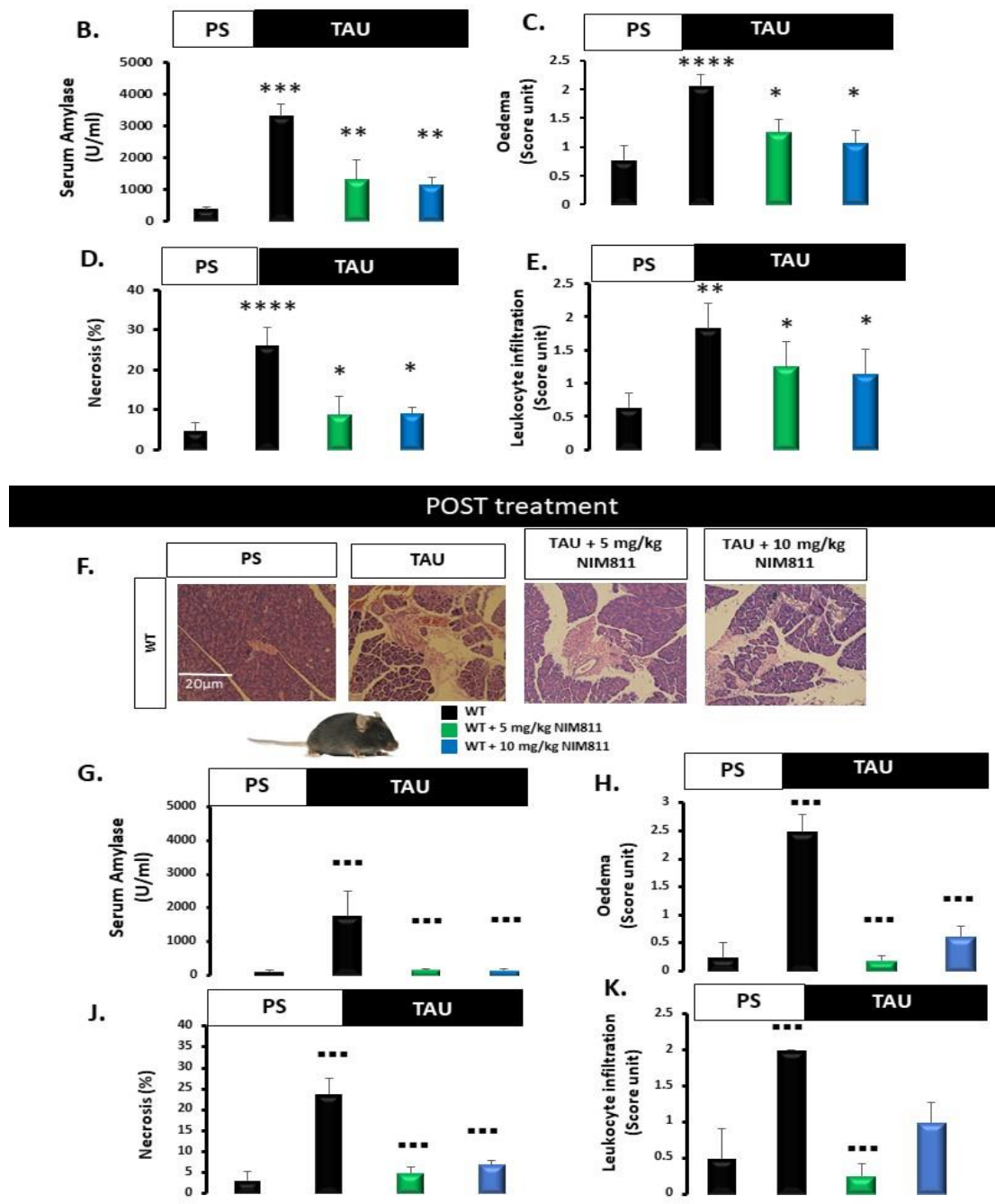

Figure8. NIM811 reduces the severity of TAU induced AP in mice. 
We performed TAU induced pancreatitis(Fig.8A-K), serum amylase measurements revealed that due to retrogrode infusion of TAU elevated serum amylase levels occured $(* * * p<0.01 \mathrm{WT}$ PS vs WT TAU Fig.6.B, - - p $<0.001$ WT PS vs WT TAU Fig.8G) howewer 5 mg/bwkg or 10 $\mathrm{mg} /$ bwkg NIM811 treatment significantly reduced the enzyme levels both in the pre and post treatment (Fig. $8 \mathrm{~B} * * \mathrm{p}<0.02$ WT TAU vs pre $5 \mathrm{mg} / \mathrm{kg}$ NIM811+TAU , ${ }^{* *} \mathrm{p}<0.02$ WT TAU vs pre $10 \mathrm{mg} / \mathrm{kg}$ NIM811+TAU, , $\cdot-\mathrm{p}<0.001$ WT TAU vs. post 5mg/kg NIM811 TAU, $\cdots p<0.001$ WT TAU vs post 10mg/kg NIM811 + TAU ) the serum amylase levels were reduced compared to WT TAU treated groups (Fig.8B. and 7G *p<0.01 WT TAU vs. WT 5mg/bwkg NIM811 TAU and ${ }^{*} p<0.01$ WT TAU vs WT 10 mg/bwkg NIM811 TAU) . During pre NIM811 treatment oedema, necrosis and leukocyte infiltration scores were significantly decreased compared to the only TAU treated groups (Fig.8A,C,D,E $\mathrm{p}<0.05$ WT TAU vs pre 5mg/bwkg NIM811 TAU/10mg/bwkg NIM811 TAU). Post insult administration of NIM811 decreased oedema, leukocyte infiltration and necrosis levels in the TAU group $(\cdots p<0.001$ Fig.8G-K) $n=4-6$ animals per group, data means \pm SEM).

\section{VI.2. Results from publication No.2.}

\section{VI.2.1. Aquaporin1 and CFTR expression in mouse pancreas}

We performed immunostaining on the pancreas samples from AQP1 and CFTR KO mice in order to characterize the possible relation between the two channels. In WT mice, AQP1 expression was detected throughout the whole plasma membrane, whereas expression of CFTR exclusively localized to the apical membrane of the ducts (Fig.9A). The absence of AQP1 caused a slight but not significant decrease in the expression of CFTR, indicating that the impaired $\mathrm{HCO}_{3}{ }^{-}$secretion in the AQP1 KO mice is not due to the decreased expression of CFTR (Fig.9A). Interestingly, we have found that expression of AQP1 significantly decreased in the ducts of CFTR KO mice, especially at the apical membrane (Fig. 9A and Fig. 9B). During this study, we have also found that the absence of CFTR did not affect the expression of AQP1 in the blood vessels indicating that some kind of interaction may exist between these two channels in the pancreatic ductal cells (data is not part of the dissertation). Although further investigations are needed to clarify whether the two channels are able to regulate each others function, expression or trafficking. 

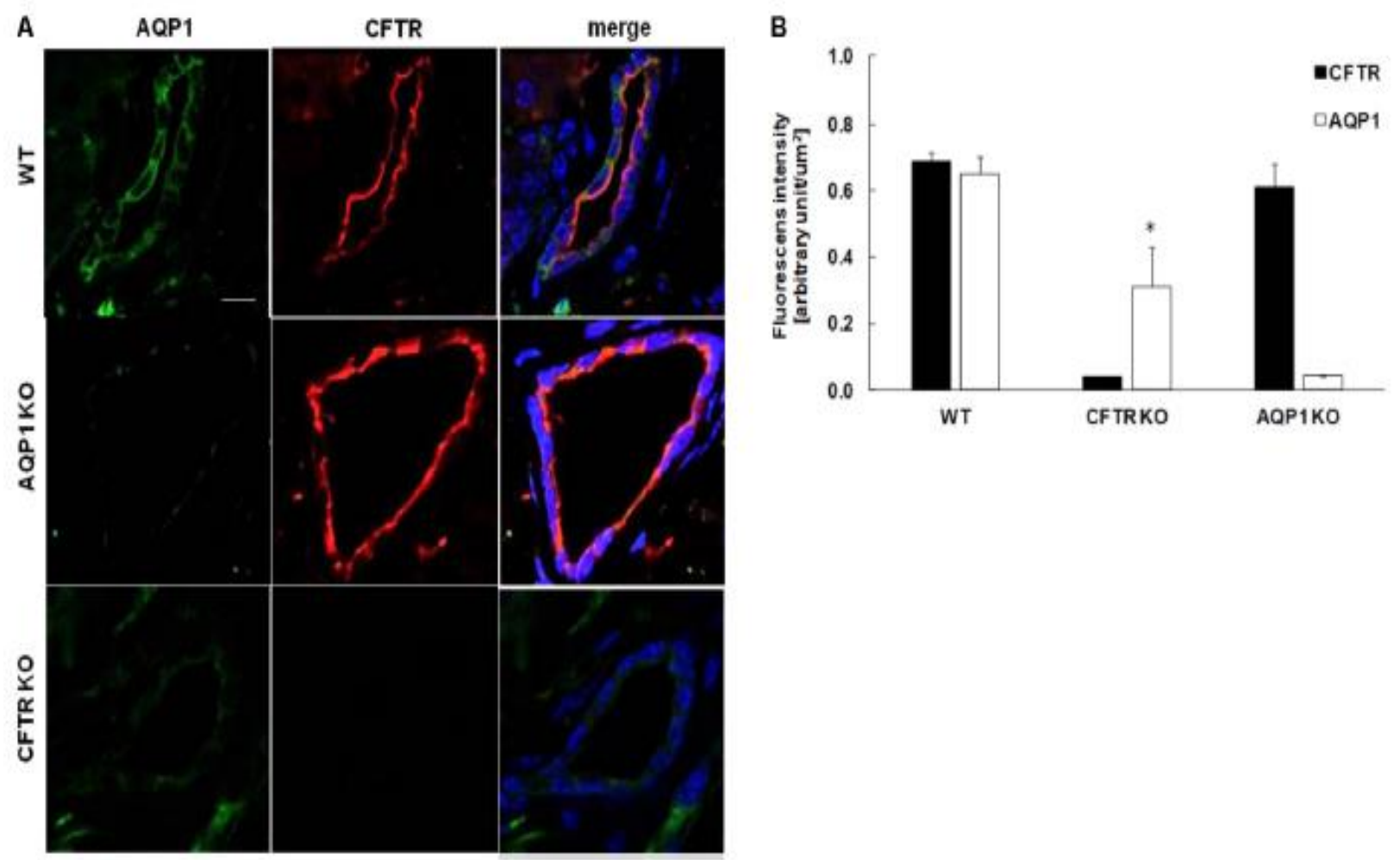

Figure 9. Expression of Aquaporin1 and CFTR in mouse pancreas. Expression of AQP1 and CFTR in the pancreas of KO. (A) Representative immunofluorescence staining of AQP1 and CFTR in wild type, AQP1 knock out and CFTR KO mice. (B) Summary bar chart shows the mean fluorescence intensity in the ductal cells normalized to the ductal area and expressed in arbitrary. Scale bar represents $10 \mathrm{~mm}$. Data are presented as means \pm SEM. *p $<0.05$ vs. WT (AQP1), $\mathrm{n}=5$. 


\section{VI.3. Results from publication No.3.}

\section{VI.3.1. Experimental modeling of MA in mice}

As an induction of metabolic acidosis, we performed different types (oral, i.p. or both) of $\mathrm{NH}_{4} \mathrm{Cl}^{-}$administration in mice.

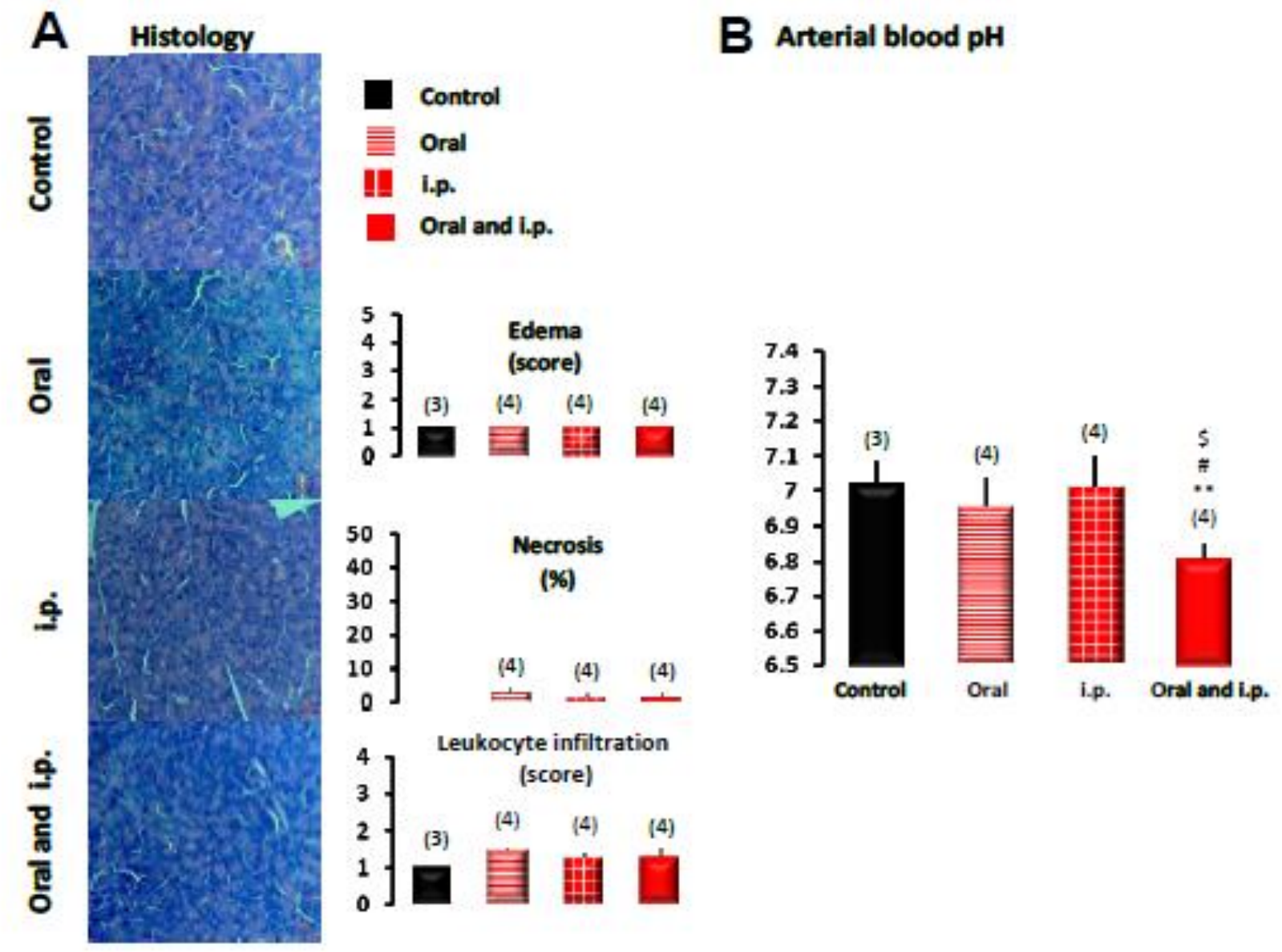

Figure 10: Development of a new method for metabolic acidosis

Induction of metabolic acidosis were performed by different administration of (oral, i.p. or both) of $\mathrm{NH} 4 \mathrm{Cl}$ in animals. None of the treatments caused pancreatic edema or necrosis, and only minimal (not significant) increase of leukocyte infiltration was observed in all three $\mathrm{NH} 4 \mathrm{Cl}$ treatment groups compared to the controls. (B) Arterial blood $\mathrm{pH}$ decreased minimally in the oral or i.p. treatment groups, while the combined (oral and i.p.) treatment significantly decreased arterial $\mathrm{pH} .{ }^{* *}, \mathrm{p}<0.01$ for the control group versus the oral and i.p. group; \#, $\mathrm{p}<$ 0.05 for the oral group versus the oral and i.p. group; and $\$, p<0.05$ for the i.p. group versus the oral and i.p. group. Scale bar represents $20 \mu \mathrm{m}$. In figures 10-12. numbers in parentheses mean the number of animals in the corresponding groups. 

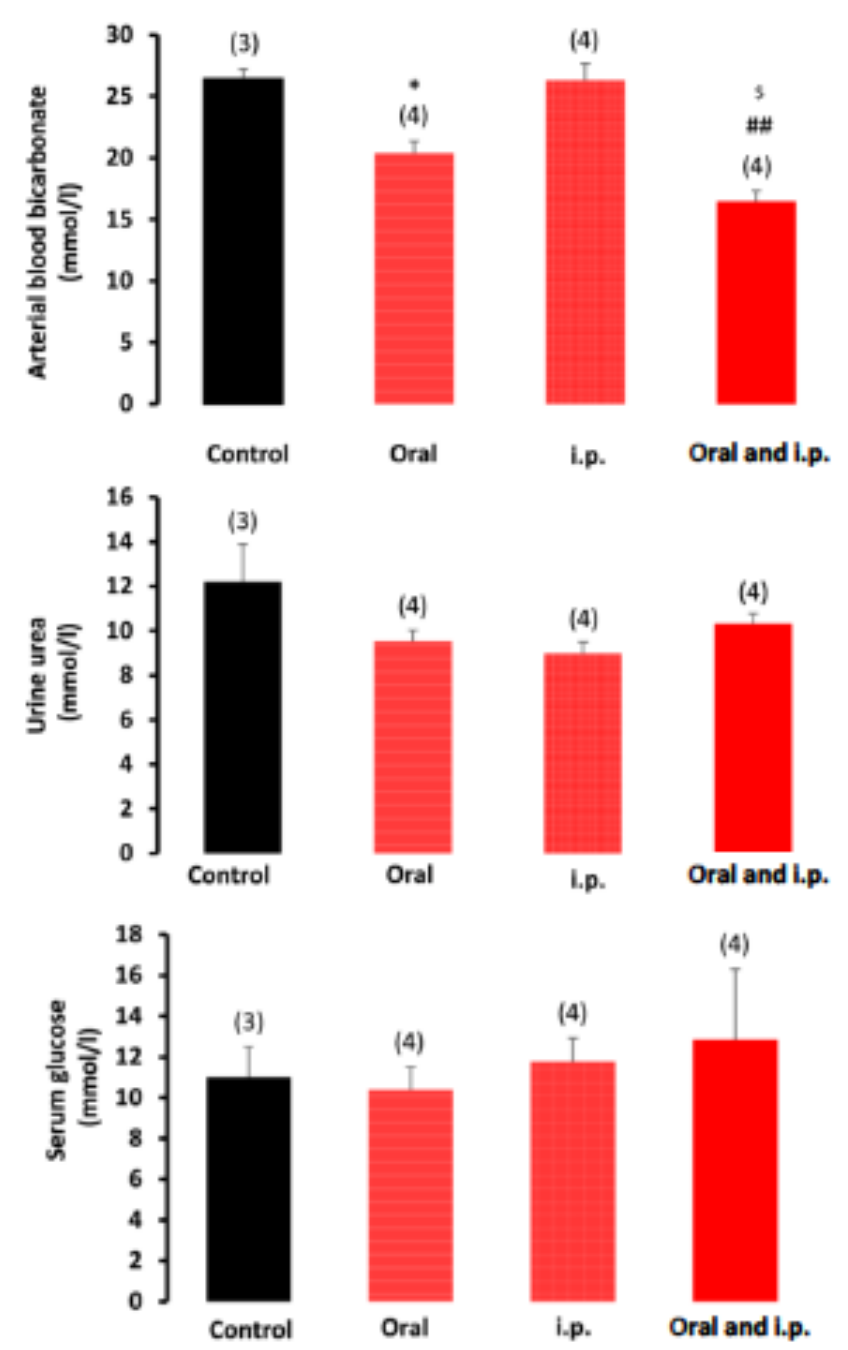

Figure 11. Laboratory parameters during the development MA groups

Scale bars represents arterial blood bicarbonate, urine urea, and serum glucose levels after different types (oral, i.p. or both) of $\mathrm{NH}_{4} \mathrm{Cl}$ administration in mice. Minimal decrease of arterial blood bicarbonate level were detected in the i.p. treatment group, while the oral and the combined (oral and i.p.) treatment significantly decreased arterial bicarbonate level were observed. Urine urea and serum glucose and levels were not changed significantly in the treatment groups. $(* \mathrm{p}<0.05$ control vs. the oral group; \#\#, $P<0.001$ e control vs. the oral and i.p. group; and $\$, P<0.05$ oral group vs. oral and i.p. group.

\section{VI.3.2. Pre-existing Acidosis Deteriorates Both Mild and Severe Forms of AP in Mice}

To determine whether the presence of pre-existing MA has any effects on the outcome of AP, pancreatic oedema, necrosis, and leukocyte infiltration scores were quantified in pancreatic sections of mice without AP, or with MAP or SAP in the presence and the absence of preexisting MA. MA caused no pancreatic damage in the control group (no AP) (Fig. 12.), which 
is line with our previous results (Fig.10-11.). On the other hand, in mice with preexisting MA, MAP resulted in significantly larger edema $(\mathrm{p}<0.05)$, increased necrosis $(\mathrm{p}<0.05)$, and elevated leukocyte infiltration $\mathrm{p}<0.05)$ compared to MAP in mice with normal blood $\mathrm{pH}$ (Fig.12). Pancreatic damage was also more severe in SAP with pre-existing MA compared to SAP in mice with normal blood $\mathrm{pH}$ as indicated by increased edema $\mathrm{p}<0.05$ ), necrosis $\mathrm{p}<$ 0.01), leukocyte infiltration $\mathrm{p}<0.05)$, and serum amylase activity $\mathrm{P}<0.05)$ (Fig.12.).

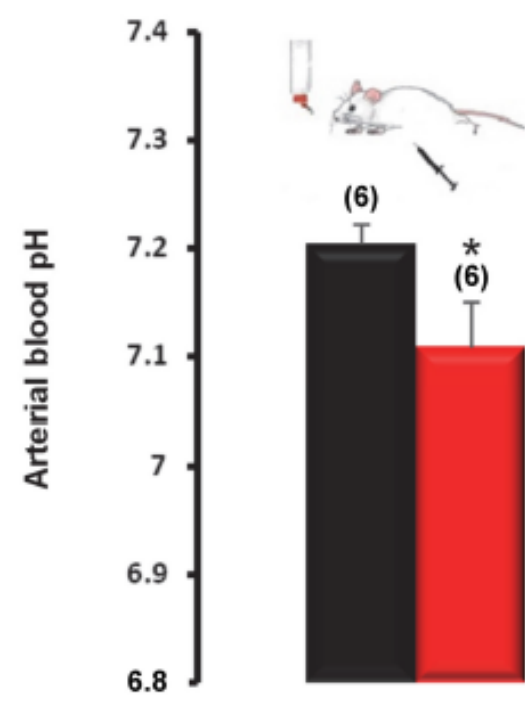

No AP

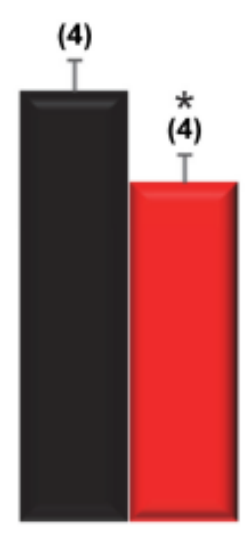

MAP

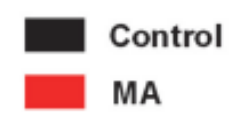

(4)

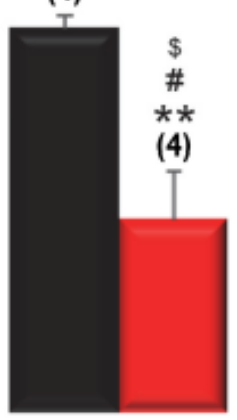

SAP

Figure 12. Arterial pH with MA induced by combination of oral and i.p. $\mathrm{NH}_{4} \mathrm{Cl}^{-}$treatment and without acidifying treatment (control groups). Mild acute pancreatitis (MAP) or severe acute pancreatitis (SAP) was induced by alcohol and fatty acid or cerulein, respectively. Mice in the control group (no AP) were injected i.p. with saline. Statistically significant differences are marked with *between MA and control (non-acidotic)groups, with \#between no AP and MAP groups in MA, and with \$between no AP and SAP groups in MA, as follows: *p $<0.05$ and ${ }^{* *} \mathrm{p}<0.01$ for MA vs. control in no AP, MAP, and SAP; $\mathrm{p}<0.05$ for no AP in MA vs. SAP in MA; $\$ p<0.05$ for MAP in MA vs. SAP in MA. 

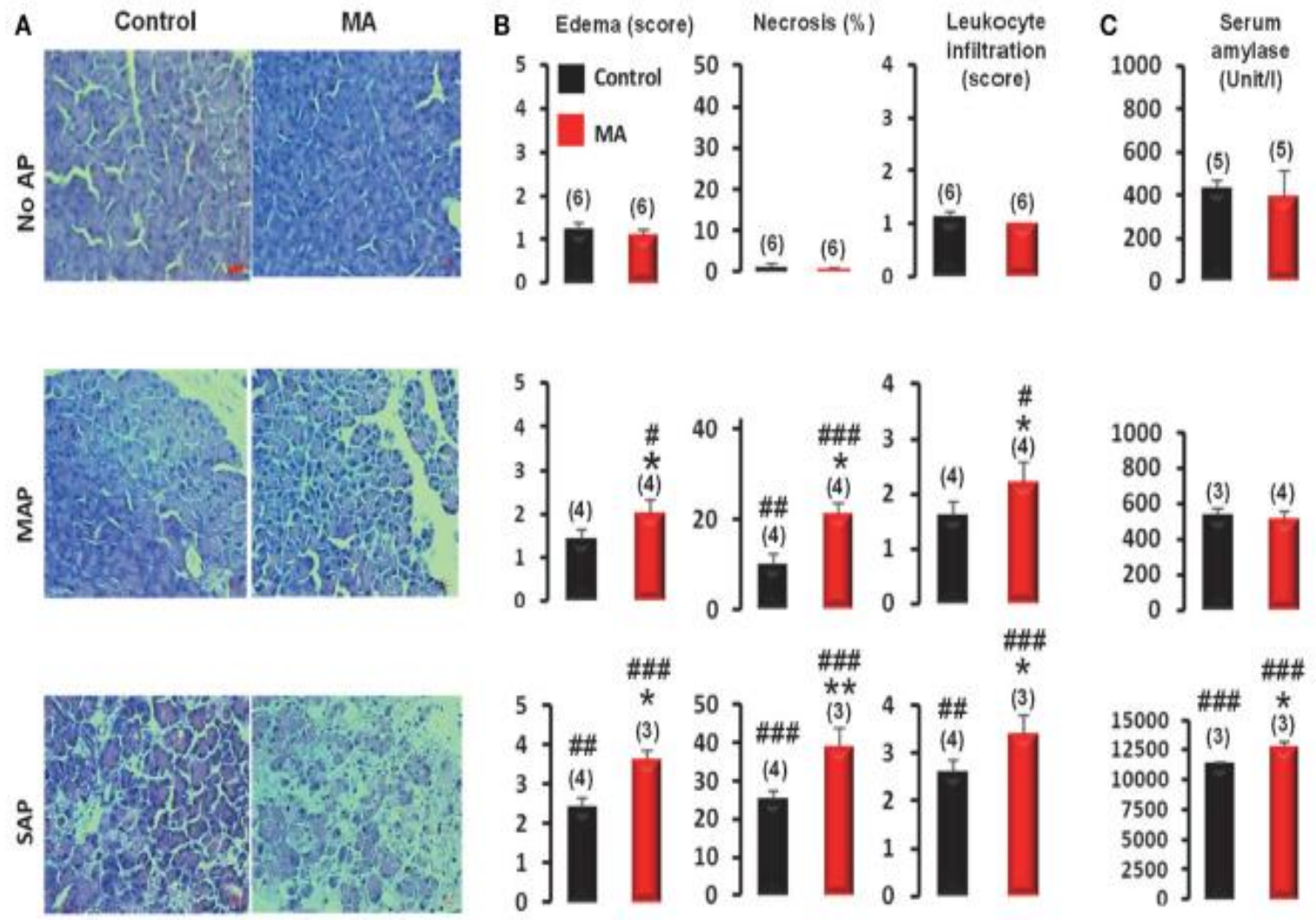

Figure 13. MA deteriorates AP in mice Representative images of pancreatic slices from mice (A), histological evaluation of oedema scores, necrosis, and leukocyte infiltration scores (B), and levels of serum amylase in MA and control mice with mild acute pancreatitis (MAP), severe acute pancreatitis (SAP) or without acute pancreatitis (no AP) (C). Scale bar represents $20 \mu \mathrm{m} . * \mathrm{p}<0.05 ; * * \mathrm{p}<0.01$ for MA vs. control in MAP and SAP; \#p $<0.05$, \#\#p $<0.01$, and $\# \#$ \# $<0.001$ for no AP vs. MAP and SAP. 


\section{DISCUSSION}

\section{VII.1. Protecting the mitochondrial homeostasis as a novel therapeutic option in AP-} Publication No.1.

Dysfunction of mitochondria is one of the main pathophysiological events in the early phase of AP in pancreatic ducts and acinar cells as well ${ }^{[10,28,85]}$. It decreases ATP production, causing elevation of intracellular calcium concentration; moreover, it negatively influences ATPdependent $\mathrm{Cl}^{-} \mathrm{HCO}_{3}{ }^{-}$exchangers, CFTR $\mathrm{Cl}^{-}$channels in ductal cells and enzyme secretory processes in acinar cells ${ }^{[28,30,32,50,85-87]}$. Henceforth, mitochondrial damage is the main factor in determining cell death pathways necrosis and apoptosis. Release of mitochondrial cytochrome $\mathrm{c}$ into the cytosol causes apoptosis, whereas mitochondrial depolarization leads to necrosis $^{[88]}$. Inhibition of mPTP could prevent both cell death mechanisms in DEC, which is different from that seen in acinar cells, where only necrosis could have been prevented. Taking it together, inhibition of mPTP seems to be beneficial in both cell types. In the last decade, it has been proved that genetic or pharmacological inhibition of mPTP reduces BA- or EtOH+FAinduced AC damage as well as augmenting the severity of AP ${ }^{[27,30,32,89]}$. In the last few years our research group revealed that both $\mathrm{BA}$ and $\mathrm{EtOH}+\mathrm{FA}$ induce inhibition of $\mathrm{HCO}_{3}{ }^{-}$secretion via severe mitochondrial damage in PDEC [50, 86][50, 85,36, 70]. During our studies we have continued the experiments investigating the role of $\mathrm{mPTP}$ and its inhibition in pancreatic ductal epithelial cells. In the first step, we characterized the role of mPTP (both genetic and pharmacological CyA) inhibition in PDEC and found that its inhibition has a strong protective effect against the toxic effects of $\mathrm{BA}$ or $\mathrm{EtOH}+\mathrm{FA}$ in ductal cells, suggesting that targeting mPTP may have general benefits. Although many mPTP inhibitors have been tested, none of them have been successful. CyA itself inhibits calcineurin, which leads to immunosuppressant activity and thus could negatively affect the treatment of patients due to hazardous infections. Clinical testing of non-immunosuppressive CyA derivatives was also stopped before reaching the "proof of concept" phase 2 clinical trials in AP because of its inconsistent behavior in other trials due to the facts noted in the introduction. Recently, other new mPTP inhibitors have been introduced in experimental studies, until now with not much success. For example, isoxazoles had inconsistent effects in myocardial infarction ${ }^{[39]}$ benzamides resulted in impaired ATP generation $^{[33,39]}$ cinnamic anilides were shown to be effective in myocardial infarction ${ }^{[90]}$.However, lately it has turned out that it has an age-related toxicity ${ }^{[91]}$. As mention in the introduction section, Nim811 has been shown to be protective in several experimental studies in different diseases, and until now no toxic effects have been demonstrated. We continued our 
study by testing the effects of NIM811 on ductal and acinar cells in vitro (results from acinar cells are not part of this dissertation). We revealed that NIM811 reduces the mitochondrial damage caused by BA or EtOH+FA. Importantly, NIM811 decreased apoptosis levels during $\mathrm{BA}$ or EtOH+FA treatment in ductal cells. Surprisingly, inhibition of mPTP protected pancreatic ductal bicarbonate but fluid secretion during $\mathrm{BA}$ or EtOH+FA treatment. Considering these results, it is assumed that rescuing intracellular ATP level and the activity of $\mathrm{Na}+\mathrm{K}+-\mathrm{ATPase}$ do not result in overall protection alone and other fluid transport mechanisms such as aquaporins may remain diminished ${ }^{[77]}$. Per os administration of 5 or $10 \mathrm{mg} / \mathrm{kg}$ NIM811 treatment alone had no toxic effect, but significantly reduced the severity of AP.

\section{VII.2. The role of AQP1 in pancreatic ductal fluid secretion- Publication No.2.}

Concerning, the AQPs role in the pancreatic ductal fluid secretion, by using double immunostaining of AQP1 and CFTR we have shown for the first time that AQP1 and CFTR are co-localized at the apical membrane of pancreatic ductal cells. Lack of CFTR significantly reduced the expression of AQP1, these data indicate that CFTR may control the water permeability of ductal cells. Our results also indicate that AQP1 interacts with the CFTR $\mathrm{Cl}^{-}$channel and takes part in the formation of pancreatic fluid. Moreover, we have found that AQP1 plays role in the pathology of pancreatitis. Earlier, similar results have been found in respiratory epithelial cells, where the CFTR channel was mutant or inhibited the water permeability of the epithelial cells significantly decreased ${ }^{[51,92]}$. This could highlight the significance of this water channel in disease of pancreatitis moreover in cystic fibrosis as well.

\section{VII.3. The vicious cycle between reduced blood pH and AP-Publication No.3.}

Since, in the literature there were no mouse model of MA, first we performed several methods of experiments to find the most beneficial MA model to use. Dual administration (oral and i.p.) of acidic fluid induced a marked $\mathrm{pH}$ drop in the blood without damaging the pancreas. By the oral treatment with i.p. acidification, our model is similar to such conditions, when primarily the $\mathrm{pH}$ of the peritoneal fluid is reduced such as bacterial peritonitis, carbon dioxide insufflation during laparoscopy ${ }^{[93]}$, or peritoneal dialysis ${ }^{[94]}$ In our model of MA , the MA manifested slowly and occured for several days in the mice which is very similar to what is happening in patients with MA. Furthermore, in human patients AP can manifest in pre-existing MA, for

instance during hyperlipidemia or diabetic ketoacidosis ${ }^{[95,96]}$ However, in clinical settings MA typically occurs as a consequence of AP and in most cases it does not pre-exist.

In the future, it should be a great goal of clinical trials to find the beneficial effects of controlled $\mathrm{pH}$ management and to search for the optimal fluid resuscitation forms in patients with AP and 
pre-existing MA. During our experiments we have shown experimental evidence to a bilateral link between $\mathrm{pH}$ and AP. We showed that pre-existing MA worsens the outcome of AP, whereas AP reduces $\mathrm{pH}$ in the blood which vicious cycle could be one of the main reasons for the high mortality rate in severe cases of AP. Future evaluations are needed to reveal the exact mechanism of how MA can deteriorate AP, but assumably a complex regulatory mechanisms is involved.

\section{SUMMARY}

\section{VIII.1. Conclusions, new therapeutic options in the treatment of AP}

1. NIM811 is a suitable compound to be tested in clinical trials of AP. We provided strong evidence that one of the mPTP inhibitors, namely NIM811 is highly effective in different experimental pancreatitis models. Since NIM811 had no side-effects and passed the important phase 1 stage in the clinical trial process, companies should organize phase 2 clinical trials with the use of this novel and promising drug candidate. (Publication No.1.)

2. Protecting fluid secretion could be a new therapeutic option in AP. AQP1 and CFTR channels are co-localized in the pancreatic ducts, we hypothesize that absence of the channel makes the pancreas more sensitive to pancreatitis, probably due to the decreased pancreatic fluid and $\mathrm{HCO}_{3}{ }^{-}$secretion. (Publication No.2.)

3. Restoring the normal $\mathbf{p H}$ in patients with AP could be a beneficial therapeutic application in the treatment of the disease. (Publication No.3.)

\section{MY OWN WORK}

Publication No.1.: I was involved in: i) the study design, ii) article search iii) data extraction and statistical analysis, iv) experimental design v) performing of all types of the used experiments - in vivo and in vitro vi) developing the data interpretation. Also, I wrote the first version of the article and took part in developing the final version. I also prepared the version 1 of the "answers to the reviewers" and the revision of the article.

Publication No.2.: I was involved in: i) performing immunofluorescent stainings of mouse pancreas samples ii) quantification of the fluorescent data (from immunofluorescent stainings) iii, statistical analysis vi, data interpretation 
Publication No.3.: I was involved in: i) the experimental study design, ii) experimental article search, iii) experimental data extraction and statistical analysis, iv) performing of all types of the used experiments - in vivo and in vitro vi) developing the experimental data interpretation. I also wrote the experimental part of the article. 


\section{TABLES}

Table1. Solutions used during our studies

\begin{tabular}{|c|c|c|c|c|c|}
\hline & $\begin{array}{c}\text { HEPES } \\
\text { (Standard) } \mathrm{mM}\end{array}$ & $\begin{array}{c}\mathrm{HCO}_{3-} \\
\text { (Standard) } \mathrm{mM}\end{array}$ & $\begin{array}{c}\mathrm{NH}_{4} \mathrm{Cl}^{-} \mathrm{HCO}_{3}- \\
\mathrm{mM}\end{array}$ & 1xTBS mM & $\begin{array}{c}\text { HBSS } \\
\text { (Standard) } \mathrm{mM}\end{array}$ \\
\hline $\mathrm{NaCl}$ & 140 & 115 & 95 & 150 & 0.137 \\
\hline KCI & 5 & 5 & 5 & - & 5.4 \\
\hline $\mathrm{CaCl}_{2}$ & 1 & 1 & 1 & - & 0.3 \\
\hline $\mathrm{MgCl}_{2}$ & 1 & 1 & 1 & - & - \\
\hline Glucose & 10 & 10 & 10 & - & 6 \\
\hline HEPES & 10 & - & - & - & - \\
\hline $\mathrm{NaHCO}_{3}-$ & - & 25 & 25 & - & 4.2 \\
\hline $\mathrm{NH}_{4} \mathrm{Cl}^{-}$ & - & - & 20 & - & - \\
\hline Trisma Base & - & - & - & 50 & - \\
\hline $\mathrm{Na}_{2} \mathrm{HPO}_{4}$ & - & - & - & - & 0.25 \\
\hline $\mathrm{KH}_{2} \mathrm{PO}_{4}$ & - & - & - & - & 0.44 \\
\hline $\mathrm{MgSO}_{4}$ & - & - & - & - & 1.03 \\
\hline
\end{tabular}




\section{REFERENCES}

1. Hegyi, P., et al., Pancreatic ductal bicarbonate secretion: challenge of the acinar Acid load. Front Physiol, 2011. 2: p. 36.

2. Pallagi, P., P. Hegyi, and Z. Rakonczay, Jr., The Physiology and Pathophysiology of Pancreatic Ductal Secretion: The Background for Clinicians. Pancreas, 2015. 44(8): p. 1211-33.

3. Steward, M.C., H. Ishiguro, and R.M. Case, Mechanisms of bicarbonate secretion in the pancreatic duct. Annu Rev Physiol, 2005. 67: p. 377-409.

4. Venglovecz, V., et al., Effects of bile acids on pancreatic ductal bicarbonate secretion in guinea pig. Gut, 2008. 57(8): p. 1102-12.

5. Pallagi, P., et al., Trypsin reduces pancreatic ductal bicarbonate secretion by inhibiting CFTR CI() channels and luminal anion exchangers. Gastroenterology, 2011. 141(6): p. 2228-2239 e6.

6. Fagenholz, P.J., et al., Increasing United States hospital admissions for acute pancreatitis, 19882003. Ann Epidemiol, 2007. 17(7): p. 491-7.

7. Fagenholz, P.J., et al., National study of United States emergency department visits for acute pancreatitis, 1993-2003. BMC Emerg Med, 2007. 7: p. 1.

8. Peery, A.F., et al., Burden of gastrointestinal disease in the United States: 2012 update. Gastroenterology, 2012. 143(5): p. 1179-1187 e3.

9. Parniczky, A., et al., Prospective, Multicentre, Nationwide Clinical Data from 600 Cases of Acute Pancreatitis. PLoS One, 2016. 11(10): p. e0165309.

10. Hegyi, P. and O.H. Petersen, The exocrine pancreas: the acinar-ductal tango in physiology and pathophysiology. Rev Physiol Biochem Pharmacol, 2013. 165: p. 1-30.

11. Sahin-Toth, M. and P. Hegyi, Smoking and Drinking Synergize in Pancreatitis: Multiple Hits on Multiple Targets. Gastroenterology, 2017. 153(6): p. 1479-1481.

12. Peery, A.F., et al., Burden of Gastrointestinal, Liver, and Pancreatic Diseases in the United States. Gastroenterology, 2015. 149(7): p. 1731-1741 e3.

13. Szentesi, A., et al., Multiple Hits in Acute Pancreatitis: Components of Metabolic Syndrome Synergize Each Other's Deteriorating Effects. Front Physiol, 2019. 10: p. 1202.

14. Halasz, A., et al., Outcomes and timing of endoscopic retrograde cholangiopancreatography for acute biliary pancreatitis. Dig Liver Dis, 2019. 51(9): p. 1281-1286.

15. Marta, K., et al., Aging and Comorbidities in Acute Pancreatitis I: A Meta-Analysis and Systematic Review Based on 194,702 Patients. Front Physiol, 2019. 10: p. 328.

16. Szakacs, Z., et al., Aging and Comorbidities in Acute Pancreatitis II.: A Cohort-Analysis of 1203 Prospectively Collected Cases. Front Physiol, 2018. 9: p. 1776.

17. Dobszai, D., et al., Body-mass index correlates with severity and mortality in acute pancreatitis: A meta-analysis. World J Gastroenterol, 2019. 25(6): p. 729-743.

18. Kiss, L., et al., The effect of serum triglyceride concentration on the outcome of acute pancreatitis: systematic review and meta-analysis. Sci Rep, 2018. 8(1): p. 14096.

19. Miko, A., et al., Preexisting Diabetes Elevates Risk of Local and Systemic Complications in Acute Pancreatitis: Systematic Review and Meta-analysis. Pancreas, 2018. 47(8): p. 917-923.

20. Nemeth, B.C., et al., Misfolding cationic trypsinogen variant p.L104P causes hereditary pancreatitis. Gut, 2017. 66(9): p. 1727-1728.

21. Godi, S., et al., Centralized care for acute pancreatitis significantly improves outcomes. J Gastrointestin Liver Dis, 2018. 27(2): p. 151-157.

22. Farkas, N., et al., A Multicenter, International Cohort Analysis of 1435 Cases to Support Clinical Trial Design in Acute Pancreatitis. Front Physiol, 2019. 10: p. 1092.

23. Parniczky, A., et al., Antibiotic therapy in acute pancreatitis: From global overuse to evidence based recommendations. Pancreatology, 2019. 19(4): p. 488-499.

24. Miko, A., et al., Observational longitudinal multicentre investigation of acute pancreatitis (GOULASH PLUS): follow-up of the GOULASH study, protocol. BMJ Open, 2019. 9(8): p. e025500. 
25. Hegyi, P., et al., The acinar-ductal tango in the pathogenesis of acute pancreatitis. Gut, 2011. 60(4): p. 544-52.

26. Hegyi, P., et al., Guts and Gall: Bile Acids in Regulation of Intestinal Epithelial Function in Health and Disease. Physiol Rev, 2018. 98(4): p. 1983-2023.

27. Sah, R.P. and A. Saluja, Molecular mechanisms of pancreatic injury. Curr Opin Gastroenterol, 2011. 27(5): p. 444-51.

28. Maleth, J., et al., Central role of mitochondrial injury in the pathogenesis of acute pancreatitis. Acta Physiol (Oxf), 2013. 207(2): p. 226-35.

29. Abu-El-Haija, M., et al., Accelerating the Drug Delivery Pipeline for Acute and Chronic Pancreatitis: Summary of the Working Group on Drug Development and Trials in Acute Pancreatitis at the National Institute of Diabetes and Digestive and Kidney Diseases Workshop. Pancreas, 2018. 47(10): p. 1185-1192.

30. Biczo, G., et al., Mitochondrial Dysfunction, Through Impaired Autophagy, Leads to Endoplasmic Reticulum Stress, Deregulated Lipid Metabolism, and Pancreatitis in Animal Models. Gastroenterology, 2018. 154(3): p. 689-703.

31. Shalbueva, N., et al., Effects of oxidative alcohol metabolism on the mitochondrial permeability transition pore and necrosis in a mouse model of alcoholic pancreatitis. Gastroenterology, 2013. 144(2): p. 437-446 e6.

32. Mukherjee, R., et al., Mechanism of mitochondrial permeability transition pore induction and damage in the pancreas: inhibition prevents acute pancreatitis by protecting production of ATP. Gut, 2016. 65(8): p. 1333-46.

33. Javed, M.A., et al., TRO40303 Ameliorates Alcohol-Induced Pancreatitis Through Reduction of Fatty Acid Ethyl Ester-Induced Mitochondrial Injury and Necrotic Cell Death. Pancreas, 2018. 47(1): p. 18-24.

34. Piot, C., et al., Effect of cyclosporine on reperfusion injury in acute myocardial infarction. N Engl J Med, 2008. 359(5): p. 473-81.

35. Cung, T.T., et al., Cyclosporine before PCl in Patients with Acute Myocardial Infarction. N Engl J Med, 2015. 373(11): p. 1021-31.

36. Zeuzem, S., et al., Randomised clinical trial: alisporivir combined with peginterferon and ribavirin in treatment-naive patients with chronic HCV genotype 1 infection (ESSENTIAL II). Aliment Pharmacol Ther, 2015. 42(7): p. 829-44.

37. Stanciu, C., et al., Efficacy and safety of alisporivir for the treatment of hepatitis $C$ infection. Expert Opin Pharmacother, 2019. 20(4): p. 379-384.

38. Atar, D., et al., Effect of intravenous TRO40303 as an adjunct to primary percutaneous coronary intervention for acute ST-elevation myocardial infarction: MITOCARE study results. Eur Heart J, 2015. 36(2): p. 112-9.

39. Sileikyte, J. and M. Forte, Shutting down the pore: The search for small molecule inhibitors of the mitochondrial permeability transition. Biochim Biophys Acta, 2016. 1857(8): p. 1197-1202.

40. Arai, M., et al., Resistance to cyclosporin A derives from mutations in hepatitis $C$ virus nonstructural proteins. Biochem Biophys Res Commun, 2014. 448(1): p. 56-62.

41. Readnower, R.D., et al., Post-injury administration of the mitochondrial permeability transition pore inhibitor, NIM811, is neuroprotective and improves cognition after traumatic brain injury in rats. J Neurotrauma, 2011. 28(9): p. 1845-53.

42. Garbaisz, D., et al., Attenuation of skeletal muscle and renal injury to the lower limb following ischemia-reperfusion using mPTP inhibitor NIM-811. PLoS One, 2014. 9(6): p. e101067.

43. Rehman, H., et al., NIM811 prevents mitochondrial dysfunction, attenuates liver injury, and stimulates liver regeneration after massive hepatectomy. Transplantation, 2011. 91(4): p. 40612.

44. Huang, Z.L., et al., Cyclophilin inhibitor NIM811 ameliorates experimental allergic encephalomyelitis. J Neuroimmunol, 2017. 311: p. 40-48.

45. Liu, Q., et al., Small-for-Size Liver Transplantation Increases Pulmonary Injury in Rats: Prevention by NIM811. HPB Surg, 2012. 2012: p. 270372. 
46. Lawitz, E., et al., Safety, pharmacokinetics, and antiviral activity of the cyclophilin inhibitor NIM811 alone or in combination with pegylated interferon in HCV-infected patients receiving 14 days of therapy. Antiviral Res, 2011. 89(3): p. 238-45.

47. Hegyi, P. and Z. Rakonczay, Insufficiency of electrolyte and fluid secretion by pancreatic ductal cells leads to increased patient risk for pancreatitis. Am J Gastroenterol, 2010. 105(9): p. 211920.

48. Takacs, T., et al., Intraductal acidosis in acute biliary pancreatitis. Pancreatology, 2013. 13(4): p. 333-5.

49. Pallagi, $P$., et al., The role of pancreatic ductal secretion in protection against acute pancreatitis in mice*. Crit Care Med, 2014. 42(3): p. e177-88.

50. Maleth, J., et al., Alcohol disrupts levels and function of the cystic fibrosis transmembrane conductance regulator to promote development of pancreatitis. Gastroenterology, 2015. 148(2): p. 427-39 e16.

51. Schreiber, R., et al., The cystic fibrosis transmembrane conductance regulator activates aquaporin 3 in airway epithelial cells. J Biol Chem, 1999. 274(17): p. 11811-6.

52. Cheung, K.H., et al., Synergistic effects of cystic fibrosis transmembrane conductance regulator and aquaporin-9 in the rat epididymis. Biol Reprod, 2003. 68(5): p. 1505-10.

53. Jesus, T.T., et al., Aquaporin-4 as a molecular partner of cystic fibrosis transmembrane conductance regulator in rat Sertoli cells. Biochem Biophys Res Commun, 2014. 446(4): p. 1017-21.

54. Burghardt, B., et al., Distribution of aquaporin water channels AQP1 and AQP5 in the ductal system of the human pancreas. Gut, 2003. 52(7): p. 1008-16.

55. Hua, Y., et al., Physiological and pathological impact of AQP1 knockout in mice. Biosci Rep, 2019. 39(5).

56. $\mathrm{Ma}, \mathrm{T}$. , et al., Defective dietary fat processing in transgenic mice lacking aquaporin-1 water channels. Am J Physiol Cell Physiol, 2001. 280(1): p. C126-34.

57. Yang, B., et al., Reduced osmotic water permeability of the peritoneal barrier in aquaporin-1 knockout mice. Am J Physiol, 1999. 276(1): p. C76-81.

58. $\mathrm{Ni}$, J., et al., Aquaporin-1 plays an essential role in water permeability and ultrafiltration during peritoneal dialysis. Kidney Int, 2006. 69(9): p. 1518-25.

59. Zhang, W., et al., Novel Endothelial Cell-Specific AQP1 Knockout Mice Confirm the Crucial Role of Endothelial AQP1 in Ultrafiltration during Peritoneal Dialysis. PLoS One, 2016. 11(1): p. e0145513.

60. Vincent, J.L. and R. Moreno, Clinical review: scoring systems in the critically ill. Crit Care, 2010. 14(2): p. 207.

61. Reed, A.M., et al., Low extracellular $\mathrm{pH}$ induces damage in the pancreatic acinar cell by enhancing calcium signaling. J Biol Chem, 2011. 286(3): p. 1919-26.

62. Noble, M.D., et al., A pH-sensitive, neurogenic pathway mediates disease severity in a model of post-ERCP pancreatitis. Gut, 2008. 57(11): p. 1566-71.

63. Bhoomagoud, M., et al., Reducing extracellular $\mathrm{pH}$ sensitizes the acinar cell to secretagogueinduced pancreatitis responses in rats. Gastroenterology, 2009. 137(3): p. 1083-92.

64. Rice, M., B. Ismail, and M.T. Pillow, Approach to metabolic acidosis in the emergency department. Emerg Med Clin North Am, 2014. 32(2): p. 403-20.

65. Zhan, X.B., et al., Prevalence and risk factors for clinically significant upper gastrointestinal bleeding in patients with severe acute pancreatitis. J Dig Dis, 2015. 16(1): p. 37-42.

66. Rumbus, Z., et al., Bidirectional Relationship Between Reduced Blood pH and Acute Pancreatitis: A Translational Study of Their Noxious Combination. Front Physiol, 2018. 9: p. 1360.

67. Baines, C.P., et al., Loss of cyclophilin D reveals a critical role for mitochondrial permeability transition in cell death. Nature, 2005. 434(7033): p. 658-62.

68. Argent, B.E., et al., Morphological, biochemical and secretory studies on rat pancreatic ducts maintained in tissue culture. Q J Exp Physiol, 1986. 71(4): p. 633-48. 
69. Gout, J., et al., Isolation and culture of mouse primary pancreatic acinar cells. J Vis Exp, 2013(78).

70. Hegyi, P., et al., Measurement of intracellular pH in pancreatic duct cells: a new method for calibrating the fluorescence data. Pancreas, 2004. 28(4): p. 427-34.

71. Hegyi, P., M.A. Gray, and B.E. Argent, Substance P inhibits bicarbonate secretion from guinea pig pancreatic ducts by modulating an anion exchanger. Am J Physiol Cell Physiol, 2003. 285(2): p. C268-76.

72. Fernandez-Salazar, M.P., et al., Basolateral anion transport mechanisms underlying fluid secretion by mouse, rat and guinea-pig pancreatic ducts. J Physiol, 2004. 556(Pt 2): p. 415-28.

73. Balazs, A., et al., Ductal Mucus Obstruction and Reduced Fluid Secretion Are Early Defects in Chronic Pancreatitis. Front Physiol, 2018. 9: p. 632.

74. Schatz, G., The protein import system of mitochondria. J Biol Chem, 1996. 271(50): p. 31763-6.

75. Pfanner, N., Mitochondrial import: crossing the aqueous intermembrane space. Curr Biol, 1998. 8(8): p. R262-5.

76. Rapaport, D., Biogenesis of the mitochondrial TOM complex. Trends Biochem Sci, 2002. 27(4): p. 191-7.

77. Venglovecz, V., et al., The Importance of Aquaporin 1 in Pancreatitis and Its Relation to the CFTR Cl(-) Channel. Front Physiol, 2018. 9: p. 854.

78. Niederau, C., L.D. Ferrell, and J.H. Grendell, Caerulein-induced acute necrotizing pancreatitis in mice: protective effects of proglumide, benzotript, and secretin. Gastroenterology, 1985. 88(5 Pt 1): p. 1192-204.

79. Ding, S.P., J.C. Li, and C. Jin, A mouse model of severe acute pancreatitis induced with caerulein and lipopolysaccharide. World J Gastroenterol, 2003. 9(3): p. 584-9.

80. Perides, G., et al., Experimental acute biliary pancreatitis induced by retrograde infusion of bile acids into the mouse pancreatic duct. Nat Protoc, 2010. 5(2): p. 335-41.

81. Huang, W., et al., Fatty acid ethyl ester synthase inhibition ameliorates ethanol-induced Ca2+dependent mitochondrial dysfunction and acute pancreatitis. Gut, 2014. 63(8): p. 1313-24.

82. Kui, B., et al., New insights into the methodology of L-arginine-induced acute pancreatitis. PLoS One, 2015. 10(2): p. e0117588.

83. Galicek, J., F. Seow, and J.M. Lingard, The effect of chronic acid/base disturbances on renal amino acid clearances in the rat. Aust J Exp Biol Med Sci, 1981. 59(4): p. 383-91.

84. Nowik, M., et al., Induction of metabolic acidosis with ammonium chloride ( $\mathrm{NH} 4 \mathrm{Cl}$ ) in mice and rats--species differences and technical considerations. Cell Physiol Biochem, 2010. 26(6): p. 1059-72.

85. Maleth, J. and P. Hegyi, Ca2+ toxicity and mitochondrial damage in acute pancreatitis: translational overview. Philos Trans R Soc Lond B Biol Sci, 2016. 371(1700).

86. Maleth, J., et al., Non-conjugated chenodeoxycholate induces severe mitochondrial damage and inhibits bicarbonate transport in pancreatic duct cells. Gut, 2011. 60(1): p. 136-8.

87. Judak, L., et al., Ethanol and its non-oxidative metabolites profoundly inhibit CFTR function in pancreatic epithelial cells which is prevented by ATP supplementation. Pflugers Arch, 2014. 466(3): p. 549-62.

88. Odinokova, I.V., et al., Mitochondrial mechanisms of death responses in pancreatitis. J Gastroenterol Hepatol, 2008. 23 Suppl 1: p. S25-30.

89. Gukovskaya, A.S., S.J. Pandol, and I. Gukovsky, New insights into the pathways initiating and driving pancreatitis. Curr Opin Gastroenterol, 2016.

90. Fancelli, D., et al., Cinnamic anilides as new mitochondrial permeability transition pore inhibitors endowed with ischemia-reperfusion injury protective effect in vivo. J Med Chem, 2014. 57(12): p. 5333-47.

91. Fang, J., et al., An Inhibitor of the Mitochondrial Permeability Transition Pore Lacks Therapeutic Efficacy Following Neonatal Hypoxia Ischemia in Mice. Neuroscience, 2019. 
92. Jourdain, $\mathrm{P}$. , et al., The human CFTR protein expressed in $\mathrm{CHO}$ cells activates aquaporin-3 in a cAMP-dependent pathway: study by digital holographic microscopy. J Cell Sci, 2014. 127(Pt 3): p. 546-56.

93. Duerr, F.M., D.C. Twedt, and E. Monnet, Changes in $\mathrm{pH}$ of peritoneal fluid associated with carbon dioxide insufflation during laparoscopic surgery in dogs. Am J Vet Res, 2008. 69(2): p. 298-301.

94. Farhat, K., et al., Conventional versus biocompatible peritoneal dialysis fluids: more questions than answers? NDT Plus, 2008. 1(Suppl 4): p. iv46-iv50.

95. Nair, S. and C.S. Pitchumoni, Diabetic ketoacidosis, hyperlipidemia, and acute pancreatitis: the enigmatic triangle. Am J Gastroenterol, 1997. 92(9): p. 1560-1.

96. Nair, S., D. Yadav, and C.S. Pitchumoni, Association of diabetic ketoacidosis and acute pancreatitis: observations in 100 consecutive episodes of DKA. Am J Gastroenterol, 2000. 95(10): p. 2795-800.

\section{FUNDING}

My $\mathrm{PhD}$ work was funded by a Momentum Grant from the Hungarian Academy of Sciences (LP2014-10/2014 to PH) as well as Economic Development and Innovation Operational Programme Grants and Project Grants from the National Research, Development and Innovation Office (GINOP-2.3.2-15-2016-00015, EFOP-3.6.2-16-2017-00006, K116634 to PH, K109756 to VV and PD115974 to JM and K119938 to ZR). 


\section{ACKNOWLEDGEMENTS}

Firstly, I would like to express my gratitude and thanks to my mentor and supervisor Prof. Dr. Péter Hegyi (Momentum Translational Gastroenterology Research Group, Hungarian Academy of SciencesUniversity of Szeged, Institute for Translational Medicine, University of Pécs) who provided the scientific and financial support throughout my PhD. studies. Without his outstanding scientific work and supervision this dissertation would not have been possible. I am very grateful for my other supervisor Dr. József Maléth (First Department of Medicine University of Szeged, Momentum Epithelial Cell Signalling and Secretion Research Group, Hungarian Academy of Sciences-University of Szeged), for the scientific counselling, valuable comments and methodological advices.

I would like to thank Prof. Dr. Csaba Lengyel and Prof. Dr. György Ábrahám, the current and former head of the First Department of Medicine, University of Szeged who gave me the opportunity to work at the Department. I also thank Prof. Dr. András Varró (former head of Department of Pharmachology and Pharmachotherapy, University of Szeged) for his support. I owe special thanks to Dr. Viktória Venglovecz (Department of Pharmachology and Pharmachotherapy, University of Szeged) for her scientific advices and kind support throughout the years. I also would like to thank Dr. Andrea Szentesi (First Department of Medicine University of Szeged and Institute for Translational Medicine, University of Pécs), Dr. Petra Pallagi (First Department of Medicine University of Szeged) and Prof. Dr. Zoltán Rakonczay Jr. (Department of Pathophysiology, University of Szeged) for the scientific advices during these years.

Thank you to all the members of the Cell and Physiology Laboratory at the First Department of Medicine, University of Szeged and the Hungarian Pancreatic Study Group. This dissertation would not have been possible to achieve without a great team and the assistance of Edit Magyarné Pálfi, Rea Fritz, Zsuzsanna Miklósné Árva, Tünde Pritz Béláné Horesnyi, Brigitta Kazi and †Erzsébet Zoltánné Fuksz. Special thanks to my great lab colleagues and friends (in alphabetical order) for their advices, assistance and the fun times we had together: Emese Réka Bálint, Zsolt Balla, Attila Ébert, Júlia Fanczal, Gabriella Für, Anett Lőrincz, Tamara Madácsy, Réka Molnár and Noémi Závogyán.

I am thankful to my husband János Tóth for his exceptional patience and for being my greatest cheerleader all along these years. I am beyond grateful to my brother Máté Gergely Tóth for always brighten my days; to all my family and friends for their emotional support. You are awesome! I dedicate

my dissertation work to my parents and grandparents. To my parents; Éva and Tibor Tóth, who taught me about the importance of diligence, education, hard work and integrity. To my grandfather; $\dagger$ Imre Tóth, who believed in my scientific research career even when I did not know what even science meant.

"Success is walking from failure to failure with no loss of enthusiasm." —Winston Churchill 
DOI: $10.1113 / J P 278517$

Novel mitochondrial transition pore inhibitor $\mathrm{N}$-methyl-4-isoleucine cyclosporin is a new therapeutic option in acute pancreatitis

Emese Tóth, ${ }^{1,2}$ József Maléth, ${ }^{1,3}$ Noémi Závogyán, ${ }^{1}$ Júlia Fanczal, ${ }^{1,3}$ Anna Grassalkovich, ${ }^{1,2}$ Réka Erdős, ${ }^{1}$ Petra Pallagi, ${ }^{1,3}$ Gergő Horváth, ${ }^{4}$ László Tretter, ${ }^{4}$ Emese Réka Bálint, ${ }^{5}$ Zoltán Rakonczay Jr., ${ }^{5}$ Viktória Venglovecz, ${ }^{6}$ Péter Hegyi ${ }^{2,7,8}$

${ }^{1}$ First Department of Medicine, University of Szeged, Szeged, Hungary

${ }^{2}$ Momentum Translational Gastroenterology Research Group, Hungarian Academy of Sciences-University of Szeged, Szeged, Hungary

${ }^{3}$ Momentum Epithelial Cell Signalling and Secretion Research Group, Hungarian Academy of Sciences-University of Szeged, Szeged, Hungary

${ }^{4}$ Department of Medical Biochemistry, Semmelweis University, Budapest, Hungary

${ }^{5}$ Department of Pathophysiology, University of Szeged, Szeged, Hungary

${ }^{6}$ Department of Pharmacology and Pharmacotherapy, University of Szeged, Szeged, Hungary ${ }^{7}$ Institute for Translational Medicine and First Department of Medicine, University of Pécs, Pécs, Hungary,

${ }^{8}$ Szentágothai Research Centre, University of Pécs, Pécs, Hungary

Corresponding author: Prof. Peter Hegyi, Institute for Translational Medicine, University of Pécs, 7624 Pecs, Szigeti út 12., hegyi2009@gmail.com, p.hegyi@tm-centre.org

This is an Accepted Article that has been peer-reviewed and approved for publication in the The Journal of Physiology, but has yet to undergo copy-editing and proof correction. Please cite this article as an 'Accepted Article'; doi: 10.1113/JP278517.

This article is protected by copyright. All rights reserved. 


\title{
KEYPOINTS
}

Bile acids, ethanol and fatty acids deteriorate pancreatic ductal fluid and bicarbonate secretion via mitochondrial damage, ATP depletion and calcium overload.

It is known that pancreatitis inducing factors open the membrane transition pore (mPTP) channel via cyclophilin D activation in acinar cells causing calcium overload and cell death and genetic or pharmacological inhibition of mPTP improves the outcome of acute pancreatitis in animal models.

In our study we show that genetic and pharmacological inhibition of mPTP protects mitochondrial homeostasis and cell function evoked by pancreatitis-inducing factors in pancreatic ductal cells.

Our results also reveal that the novel Cyclosporin A derivative NIM811 protects mitochondrial function in acinar and ductal cells, moreover it preserves bicarbonate transport mechanisms in pancreatic ductal cells.

We found that NIM811 is highly effective in different experimental pancreatitis models and that NIM811 has no side-effects. NIM811 is a highly suitable compound to be tested in clinical trials .

\begin{abstract}
Background and aims

Mitochondrial dysfunction plays a crucial role in the development of acute pancreatitis (AP); however, no compound is currently available with clinically acceptable effectiveness and safety. In this study, we investigated the effects of a novel mitochondrial transition pore inhibitor, N-methyl-4-isoleucine cyclosporin (NIM811), in AP.
\end{abstract}

\section{Methods}

Pancreatic ductal and acinar cells were isolated by enzymatic digestion from $\mathrm{Bl} / 6$ mice. In vitro measurements were performed by confocal microscopy and microfluorometry. Preventive effects of pharmacological (cylosporin A $(2 \mu \mathrm{M})$, NIM811 $(2 \mu \mathrm{M})$ ) or genetic (Ppif $^{-/} /$Cyp D KO) inhibition of the mitochondrial transition pore (mPTP) during the administration of either bile acids (BA) or ethanol + fatty acids (EtOH+FA) were examined. Toxicity of mPTP inhibition was investigated by detecting apoptosis and necrosis. In vivo effects of the most promising compound, NIM811 (5 or $10 \mathrm{mg} / \mathrm{kg}$ per os), were checked in 
three different AP models induced by either caerulein $(10 \times 50 \mu \mathrm{g} / \mathrm{kg})$, ethanol+ fatty acid $(1.75$ $\mathrm{g} / \mathrm{kg}$ ethanol and $750 \mathrm{mg} / \mathrm{kg}$ palmitic acid) or $4 \%$ taurocholic acid $(2 \mathrm{ml} / \mathrm{kg})$.

\section{Results}

Both genetic and pharmacological inhibition of Cyp D significantly prevented the toxic effects of $\mathrm{BA}$ and $\mathrm{EtOH}+\mathrm{FA}$ by restoring mitochondrial membrane potential $(\Delta \psi)$ and preventing the loss of mitochondrial mass. In vivo experiments revealed that per os administration of NIM811 has a protective effect in AP by reducing oedema, necrosis, leukocyte infiltration and serum amylase level in AP models. Administration of NIM811 had no toxic effects.

\section{Conclusion}

The novel mitochondrial transition pore inhibitor NIM811 seems to be an exceptionally good candidate compound for clinical trials in AP.

\section{KEYWORDS}

Acute pancreatitis, mitochondrial transition pore, cyclophilin D, NIM811

\section{INTRODUCTION}

Acute pancreatitis (AP) is among the most common gastrointestinal disorders requiring hospitalization in the United States (Fangenholz et al,2007; Fagenholz et al, 2007 ; Peery et al ,2012 ) . Although the disease is generally mild, the mortality rate in its severe form is still unacceptably high (Parniczky et al, 2016). In recent years, our understanding of the mechanisms that play a crucial role in the development of the disease has improved (Abu-ElHaija et al , 2018) . Impaired autophagy, trypsinogen activation, excessive $\mathrm{Ca}^{2+}$ influx, calcineurin activation, mitochondrial dysfunction and cystic fibrosis transmembrane conductance regulator (CFTR) inhibition were shown to have a great impact in the early phase of AP. Therefore, targeting one of these mechanisms may lead to the first specific therapy in AP. 
Among the mechanisms noted above, one of the earliest events in AP is mitochondrial dysfunction (Sah and Saluja,2011; Maleth et al, 2013; Abu-El-Haija et al, 2018 ; Biczo and Vegh et al ,2018;) . It has been shown in acinar cells that bile acids (BA) and ethanol and fatty acids $(\mathrm{EtOH}+\mathrm{FA})$ open the membrane transition pore (mPTP) channel via cyclophilin D (Cyp D) activation, keeping the channel continuously opened and thus resulting in mitochondrial depolarization, lower ATP synthesis and cell necrosis (Shalbueva et al, 2013; Mukherjee et al, 2016; Abu-El-Haija et al, 2018 ). Although it is still unknown how the pancreatitis-inducing factors noted above modify mPTP channel activity in pancreatic ductal epithelial cells (PDEC), it still seems to be one of the most promising drug targets and calls for further investigation.

Until now, cyclosporin A (CyA) is the only licenced compound used experimentally to inhibit mPTP (via Cyp D) (Javed et al, 2018); however, its clinical usefulness is highly questionable for several reasons. A pilot study found that CyA could reduce the size and damage of myocardial infarction, but larger studies showed no beneficial effects (Piot et al,2008; Cung et al,2015; Javed et al, 2018). Even efforts to decrease its immunosuppressive activity have not been successful. Moreover, CyA derivative Debio025 (Alispovirir, Debiopharm) has been found effective against the hepatitis C virus (HCV), but it had serious side-effects. Surprisingly, some of the patients developed pancreatitis, resulting in a clinical hold on the global Debio025 trial programme (Zeuzem et al,2015; Stanciu et al, 2019). Another derivative, TRO40303 (3,5-seco-4-nor-cholestan-5-one oxime-3-o, TROPHOS, Roche), was not beneficial in a phase 2 trial of cardiac preservation following acute myocardial infarction, suggesting that this compound has low or no effectivity (Atar et al, 2015) . Lately, it has turned out that TRO40303 does not even bind to Cyp D directly (Sileikyte,2016 ; Javed et al, 2018;) . With regard to AP, both Debio025 and TRO40303 have been shown to be beneficial in animal models, but neither of them have reached "proof of concept" clinical trials in AP, most probably due to the clinical failures noted above. All in all, new compounds are crucially needed.

A novel cyclosporin A derivative, $N$-methyl-4-isoleucine cyclosporin (NIM811), was found to be highly beneficial in different experimental and clinical studies. NIM811 was effective in animal models of central nervous system injury (Readnower et al, 2011), allergic encephalomyelitis (Huang et al, 2017), ischaemic-reperfusion injury after surgical intervention (Garbaisz et al,2014) ,hepatitis C (Arai et al,2014), liver transplantation 
(Rehman et al, 2011) and pulmonary injury during liver transplantation (Liu et al, 2012) . Importantly, none of the studies reported side-effects. NIM811 had no severe or serious adverse effects in a phase 2 clinical trial on HCV-infected patients, suggesting that NIM811 has no toxic immunosuppressant activity either (Lawitz et al, 2011).

In this study, we show in several in vitro and in vivo experiments that either pharmacological or genetic inhibition of Cyp D restores mitochondrial function not only in acinar cells, but also in ductal cells, highlighting the general importance of mPTP in AP. Moreover, we provide evidence that NIM811 is highly effective in different experimental pancreatitis models and that NIM811 has no side-effects.

\section{MATERIALS AND METHODS}

\section{Ethical approval}

The animal experiments were performed in compliance with European Union Directive 2010/63/EU and Hungarian Government Decree 40/2013 (II.14.). Experiments were approved by local ethics committees for investigations involving animals at the University of Szeged (XII/4988/2015). In our study all animals were euthanized by $200 \mathrm{mg} / \mathrm{kg}$ pentobarbital i.p. (Bimeda MTC, Cambridge, Canada).

\section{Animals}

A total of 70 wild type (WT) and cyclophilin D knockout (Cyp D KO, (B6;129Ppiftm1Maf/J) mice were sacrificed. Cyp D KO mice were generated by targeted disruption of the Ppif gene (which encodes the Cyp D that is a component of the mPTP) (Baines et al, 2005). Cyp D KO animals were provided for us by the Department of Medical Biochemistry, Semmelweis University, Budapest, Hungary. Wild type and Cyp D-deficient littermate mice (of C57B1/6 J background, either sex, aged between 20 and 45 days) were housed in a room maintained at $20-22^{\circ} \mathrm{C}$ on a $12 \mathrm{~h}$ light-dark cycle with food and water available ad libitum. To ensure a homologous genetic background, mice were backcrossed with C57B16/J mice for at least eight generations.

\section{Solutions and chemicals}

Chemicals were obtained from Sigma-Aldrich (Budapest, Hungary), unless otherwise stated. 2.7-bis-(2-carboxyethyl)-5-(and-6-) carboxyfluorescein-acetoxymethylester (BCECF-AM) 
and Tetramethylrhodamine-methylester (TMRM) were purchased from Termofischer Scientific . NIM811 were purchased from MedChem Express Europe (Sweden). Cyclosporin A (CYA), caerulein (CER), NIM811, CCCP and fluorescence dies were diluted in dimethyl sulfoxide (DMSO) . Table 1 describes the constitution of solutions that we used during the study. In this study $500 \mu \mathrm{M}$ Chenodeoxycholic acid (bile acid,BA) or $100 \mathrm{mM}$ ethanol (EtOH) $+200 \mu \mathrm{M}$ palmitoleic acid (fatty acid, FA) was used during the fluorescence, confocal microscopy and immunostaining measurements, to evaluate the effect of bile acids or the alcohol and fatty acid induced damage on the mitochondrial and cell function during the genetic or pharmachological inhibition of the MPTP in pancreatic ducts or acinar cells. 100 $\mu \mathrm{M}$ of Carbonyl cyanide 3-chlorophenylhydrazone (CCCP) were used in the mitochondrional measurements as a positive control for mitochondrial damage.

$2 \mu \mathrm{M}$ CYA and $2 \mu \mathrm{M}$ NIM811 were used to pharmacologically inhibit mPTP. Prior to the fluorescence and confocal microscopy, immunostainings, the cells (ducts and acinar cells as well) from the CYA- or NIM811- treated groups were pretreated for 25-30 minutes with the compounds (CYA or NIM811).

\section{Abbrevations used in this study:}

AP- acute pancreatitis, NIM811- N-metil-izoleucine cyclosporine, mPTP- mitochondrial transition pore, mitochondrial membrane potencial- $\psi$, CFTR- cystic fibrosis transmembrane conductance regulator, PDEC-pancreatic ductal epithelial cells, Cyclophylin D- Cyp D ,

CYA- cylosporin A, Hepatitis C virus-HCV, Debio025- Alispovirir, Tro40303-3,5-seco-4-norcholestan-5-one-oxime-3-0, PCR-polymerase chain reaction, TMRMTetramethylrhodamine Methyl Ester Perchlorate, TOM20- Mitochondrial import receptor subunit, FA- fatty acid (palmitoliec acid), FAEE- Fatty acid ethyl ester, ETOHethanol, BA- CDC- chenodeoxycholic acid, BCECF-AM (2',7'-Bis-(2-Carboxyethyl)-5(and-6)-Carboxyfluorescein, Acetoxymethyl Ester), CER-caerulein, TAU- sodium taurocholate, TBS- Tris Buffered Solution, BSA- Bovine Serum Albumin, HBSS- Hank1s Stock Solution, CBD- Common Pancreatic Biliary Duct, CCCP- Carbonyl cyanide 3chlorophenylhydrazone

\section{Methods}

\section{Mouse genotyping}

This article is protected by copyright. All rights reserved. 
Genotypes of cyclophilin D deficient mice were identified by PCR (typical polymerase chain reaction, analyses from tail genomic DNA) . PCR-mix contained: Taq DNA pol $5 \mathrm{U}$ and 10xTaq Buffer (Abgene, Portmouth, USA), $\mathrm{MgCl}_{2}$ 1,5 mM, dNTP 2.5mM, F-null2/LoxP1f/CyPuP2 primers $(20-20 \mu \mathrm{M}), \mathrm{dH}_{2} \mathrm{O}$ and template DNA sample. Total reactions $\operatorname{mix}$ volume was $\quad 25 \quad \mu$. The wild type allele was detected using LoxP1f, 5'-AAA CTT CTC AGT CAG CTG TTG CCT CTG-3' as a forward primer and F-null2, 5'- GCT TTG TTA TCC CAG CTG GCG C-3' as a reverse primer. For genotyping of the mutant cyclophilin D deficient allele, F-null2, 5'-TTC TCA CCA GTG CAT AGG GCT CTG -3' was used as a forward primer with the reverse primer for WT (Table 2. ). DNA was denatured at $95^{\circ} \mathrm{C}$ for 2 mins, followed by 30 cycles of amplification: $94^{\circ} \mathrm{C}$ for $30 \mathrm{secs}, 60^{\circ} \mathrm{C}$ for $30 \mathrm{secs}, 72^{\circ} \mathrm{C}$ for $45 \mathrm{secs}$ and a final primer extension step at $72^{\circ} \mathrm{C}$ for 7 mins. Bands of 270 and 470 base pairs were amplified for WT and CypDKO mice, respectively.

Pancreatic ducts and acinar cells were isolated by microdissection and enzymatic digestion as described earlier (Argent et al,1986 ; Gout et al, 2013) (Argent, Arkle et al. 1986, Gout, Pommier et al. 2013).

Mitochondrial membrane potential $(\Psi)$ were determined by Zeiss LSM 880 confocal laser scanning microscope (Carl Zeiss Technika Kft., Budaörs, Hungary). BA or EtOH + FA were used to induce mitochondrial damage. Isolated pancreatic ducts or acinar cells were incubated in standard HEPES solution and loaded with TMRM (Tetramethylrhodamine Methyl Ester Perchlorate, $100 \mathrm{nmol} / \mathrm{L})$.

In order to monitor apoptotic and necrotic cells in isolated pancreatic ducts or acinar cells an apoptosis/necrosis kit was used (ab176750, Abcam). To determinate live, necrotic or apoptotic cells, CytoCalcein Violet 450 fluorescent, Apopxin Deep Red Indicator and Nuclear Green DCS1 fluorecence dies (ab176750, Abcam) were used. Samples were incubated in the mixture of the above stated fluorescence dyes at room temperature for 30-35 mins (after 25 min treatment of with BA/ETOH+FA/CYA/NIM811) in dark prior to the confocal microscopy measuremets. In case of CYA or NIM811 treated ducts or acinar cells, the incubation with these compounds were performed before staining with the fluorescence dyes . Stainings were analyzed using a Zeiss LSM 880 confocal laser scanning microscope (Carl Zeiss Technika Kft., Budaörs, Hungary). Live, necrotic or apoptotic cells were counted and summarized in percentage of each sample, then data were summarized to average and statistical analysis was performed.

This article is protected by copyright. All rights reserved. 
Microfluorometry was used to measure pancreatic ductal $\mathrm{HCO}_{3}{ }^{-}$secretion as described earlier (Hegyi et al, 2003, Hegyi et al, 2004) by using BCECF-AM (2',7'-Bis-(2Carboxyethyl)-5-(and-6)-Carboxyfluorescein, Acetoxymethyl Ester, $1.5 \mathrm{mmol} / \mathrm{L}$ ).

Functionally active mitochondria were detected with immunofluorescent staining (TOM20 mitochondrial marker, (EPR15581-39, Abcam)). In order to determine mitochondrial localisation in isolated pancreatic ductal or acinar cells we labeled the mitochondria by the using of TOM20 primary antibody ( Abcam, EPR15581-39). TOM20 is the central unit of the receptor TOM complex in the mitochondrial outer membrane and the role of it is to recognise and translocate cytosolically synthetized mitochondrial preproteins (Shatz et al,1996; Pfanner,1998; Rapaport,2002). Isolated pancreatic ducts were frozen in cryomold at $20^{\circ} \mathrm{C}$. The cryosections (thickness $7 \mu \mathrm{m}$ ) of the isolated pancreatic ducts from WT and Cyp D KO mice were cut by Leica Cryostat. Sections were fixed in 4\% paraformaldehyde. Washing periods were administered with 1xTBS solution. Antigen retrieval was performed with $10 \mathrm{mM}$ Sodium -Citrate solution at the $\mathrm{pH}$ of 6 at $95{ }^{\circ} \mathrm{C}$ for 15 minutes. Blocking was obtained for $1 \mathrm{~h}$ with $1 \%$ goat serum in $5 \%$ BSA-TBS solution. After these sections were incubated with TOM20 rabbit monoclonal antibody (dilution 1:400,Abcam) overnight incubation at $4^{\circ} \mathrm{C}$. The following day the samples were incubated with goat anti rabbit secondary antibody (Alexa fluor 488, Thermo Fisher, Rockford, IL, United States) for 2 hours at dark in room temperature. The nuclei were counterstained with Hoechst 33342 (Termofischer, Rockford,IL,United States) . Immunofluorescence staining of the isolated pancreatic acinar cells were performed freshly after the isolation procedure with the same conditions as stated above, (except two parameters ; cells were fixed in $2 \%$ paraformaldehyde and dilution fo the primary antibody was 1:200) as stated above. Both ductal and acinar cell samples were mounted with Fluoromount and then analyzed using a Zeiss LSM 880 confocal laser scanning microscope (Carl Zeiss Technika Kft., Budaörs, Hungary). To quantify TOM20 positively stained area, 5-6 representative images from each group were taken by Zeiss LSM 880 Confocal Scannig Microscope (Carl Zeiss Technika Kft., Budaörs, Hungary). Image J software was used to convert images to gray scale (16 bit), threshold function was used to select the positively stained area. The fluorescence signal were calculated by the software (arbitary scale from 0-negative (white) to 255-maximal staining (black)) (Venglovecz et al,2018). Fluorescence intensity of the images were then normalized to the own total ductal or acinar area of the samples, which were measured in arbitary units. Fluorescence intensity was given in $\%$, normalized to the total ductal or acinar total area. 
AP was induced by caerulein $(\mathrm{CER}, 10 \times 50 \mu \mathrm{g} / \mathrm{kg}) ; 4 \%$ sodium taurocholate (TAU, 2ml/kg,4\%) (Niederau et al, 1985 ; Ding et al,2003 ; Perides et al,2010; Pallagi and Balla et al;2014;) or alcohol and fatty acid (intraperitonal injection of $1.75 \mathrm{~g} / \mathrm{kg}$ ethanol and 750 $\mathrm{mg} / \mathrm{kg}$ palmitic acid, EtOH+FA) as described earlier (Huang et al.,2014;Maleth et al, 2015). All control groups received physiological saline in the same amount as the CER, EtOH+FA or the TAU solutions respectively. Pre-treatment of the animals by NIM811 was performed and mice were gavaged orally once $1 \mathrm{~h}$ prior to the induction AP, concentrations of NIM811 were $10 \mathrm{mg} / \mathrm{kg}$ or $5 \mathrm{mg} / \mathrm{kg}$. Dosage of NIM811 was chosen according to a previous study in which NIM811 was effective against mitochondrial damage in liver transplantation (Rehman et al, 2011). Oral gavage treatment were performed by the use of plastic feeding tubes (20ga x $38 \mathrm{~mm}$, Instech Laboratories, USA). NIM811 were solubilized in a vehicle which contained $8.3 \%$ polyoxyl 40 hydrogenated castor oil and $8.3 \%$ ethanol (Rehman et al, 2011) .

NIM811 was used as a post-AP treatment as well. NIM811 was administered 12 hour after the induction of AP in the TAU or EtOH+FA induced experimental pancreatitis models. Concerning the CER induced AP, NIM811 was administered after the $3^{\text {rd }}$ injection of CER. The method for retrograde intraductal infusion of TAU has been described by Perides et al (Perides et al, 2010) . The surgery was performed on anesthetized mice (with ketaminexylazine, dosage: $87.5 \mathrm{mg} / \mathrm{kg}$ ketamine- $12.5 \mathrm{mg} / \mathrm{kg}$ xylazine). At the end of the procedure the mice were placed on a heating pad for 40 minutes and received buprenorphine i.p. injection $(0.075 \mathrm{mg} / \mathrm{kg})$ at once to reduce their occurrent pain. Following these mice were replaced into their cages for 24 hours. They had free access to food and water. 24 hours after the TAU or EtOH +FA induced AP the mice were euthanized by $200 \mathrm{mg} / \mathrm{kg}$ pentobarbital i.p. (Bimeda MTC, Cambridge, Canada), . During the CER induced AP mice were euthanized with 200 $\mathrm{mg} / \mathrm{kg}$ pentobarbital i.p. (Bimeda MTC, Cambridge, and Canada) 2 hours after the last injections of CER. Mice were exsanguinated through cardiac puncture and the pancreas were removed. Blood from the cardiac puncture was placed on ice, then centrifuged with $2500 \mathrm{RCF}$ for $15 \mathrm{mins}$ at $4^{\circ} \mathrm{C}$. Blood serum was collected from the pellet and stored at $-20^{\circ} \mathrm{C}$ until use. Pancreas samples were placed into $8 \%$ neutral formaldehyde solution and stored at $-4^{\circ} \mathrm{C}$ until the hematoxylin -eosin staining was performed. A colorimetric kit was used to measure serum amylase activity (Diagnosticum, Budapest, Hungary). Absorbance of the samples were detected at $405 \mathrm{~nm}$ with the use of FLUOstar OPTIMA (BMG Labtech, Budapest, Hungary) microplate reader. Formaldehyde-fixed pancreas samples were embedded in paraffin and were cut into $3 \mu \mathrm{m}$ thick sections and stained for hematoxylin- 
eosin by using a standard laboratory method. To quantify oedema, necrosis and leukocyte infiltration grades a semiquantitative scoring system was used as Kui et al described previously (Kui and Balla et al, 2015) .

In vitro pancreatic ductal fluid secretion (luminal swelling) assays were developed by Fernández-Salazar et al, (Fernández-Salazar et al,2004) performed by videomicroscopy as described earlier (Balázs et al,2018). Briefly, stimulaton of pancreatic ductal fluid secretion was induced by $5 \mu \mathrm{M}$ forskolin and $100 \mu \mathrm{M}$ 3-isobutyl-1-methylxanthine (IBMX), quantification were performed by Image $\mathrm{J}$ Software (Balázs et al,2018). In vivo fluid secretion measurements were performed on anesthetized (by i.p. $87.5 \mathrm{mg} / \mathrm{kg}$ ketamine- 12.5 $\mathrm{mg} / \mathrm{kg}$ xylazine ) mice after CER or EtOH+FA induced AP prior to euthanasia. Animals were placed on warm pads $(37 \circ \mathrm{C})$ to maintain the body temperature. Briefly, the abdomen of the mice were opened and cannucaltion of the lumen of the common biliopancreatic duct was performed by a 30-gauge needle (Maléth et al, 2015). Then the proximal end of the common duct was closed by a microvessel clip (Braun-Aesculap, Tuttlingen, Germany) to prevent contamination with bile, and the pancreatic juice was collected in PE-10 tube for $15 \mathrm{~min}$. In vivo secretion was induced by i.p. administration of $0.75 \mathrm{CU} / \mathrm{kg}$ secretin (Maléth et al, 2015).

\section{Statistical Analysis}

All data are expressed as means \pm SEM. Data were compared by either one- or two-way analysis of variance (ANOVA) or Kruskal-Wallis tests followed by the Holm-Sidak Method as appropriate (Sigma Plot). The effects were considered significant when $p<0.05$.

\section{RESULTS}

\section{Genetic inhibition of mPTP protects mitochondrial homeostasis and cell function evoked by pancreatitis-inducing factors in PDEC}

First, we measured the effects of the most relevant pancreatitis-inducing factors on mitochondria in primary intact ducts isolated from $\mathrm{Ppif}^{-/}$and WT mice. Experiments performed with TMRM and TOM20 revealed that genetic inhibition of mPTP decreased both the loss of $\Delta \psi$ (Fig. 1A) and mitochondrial mass (Fig. 1B) caused by $500 \mu \mathrm{M} \mathrm{CDC} \mathrm{(BA)} \mathrm{or}$ co-administration of $100 \mathrm{mM}$ ethanol and $200 \mu \mathrm{M}$ palmitoleic acid (EtOH+FA). Co-staining 
the pancreatic ducts with CytoCalcein Violet, Apopxin Deep Red and Nuclear Green showed that genetic inhibition of MPTP also decreased the extent of necrosis and apoptosis during the administration of BA or EtOH+FA (Fig. 1C), suggesting that genetic inhibition of Cyp D has a protective effect on PDEC. Next, we investigated how the genetically preserved mitochondrial function affects the cellular function of PDEC (Fig. 1D). We used the $\mathrm{NH}_{4} \mathrm{Cl}$ pulse technique, which is uniquely suited to characterizing both $\mathrm{HCO}_{3}{ }^{-}$influx and efflux mechanisms. Our experiments demonstrated that the inhibitory effects of BA and EtOH+FA on $\mathrm{Cl} / \mathrm{HCO}_{3}{ }^{-}$exchangers $\left(\mathrm{HCO}_{3}{ }^{-}\right.$efflux $)$and on $\mathrm{Na} / \mathrm{HCO}_{3}{ }^{-}$co-transporters $\left(\mathrm{HCO}_{3}{ }^{-}\right.$influx $)$are totally blocked in $\mathrm{Ppif}^{--}$vs WT mice, suggesting that inhibition of mPTP can preserve ductal function and thus has therapeutic benefits (Fig. 1D-F).

\section{Pharmacological inhibition of mPTP by CyA effectively prevents mitochondrial damage evoked by pancreatitis-inducing factors in PDEC}

Both BA and EtOH+FA significantly decreased the $\psi$ of PDEC (Fig. 2A). Importantly, $2 \mu \mathrm{M}$ CYA effectively blocked the toxic effects of the BA- and EtOH+FA-preserving function of mitochondria during the presence of pancreatitis-inducing factors. As regards the quantity of mitochondria, CYA effectively inhibited loss, as we could see during the genetic inhibition of mPTP (Fig. 2B). $2 \mu \mathrm{M}$ CYA decreased the extent of necrosis and apoptosis during the administration of BA or EtOH+FA in PDEC (Fig. 2C). Finally, we provided strong evidence of the beneficial effects of CYA on mPTP noted above, mitochondrial mass and cell death, resulting in preserved $\mathrm{HCO}_{3}{ }^{-}$efflux and influx mechanisms during BA or EtOH-FA administration (Fig. 2D-F).

\section{NIM811 treatment protects mitochondrial function and preserves bicarbonate transport mechanisms in PDEC}

Next, we investigated the effects of the novel CYA derivative NIM811 on mitochondrial function and of bicarbonate secretion on isolated pancreatic ducts. According to our data, NIM811 reduces the BA- or EtOH+FA-induced damage to mitochondrial function and morphology in isolated pancreatic ducts (Fig. 3A-B). Experiments using CytoCalcein Violet, Apopxin Deep Red and Nuclear Green showed that NIM811 alone has no toxic effects on 
PDEC. Furthermore, it can strongly decrease BA- or EtOH-FA-evoked necrosis and apoptosis (Fig. 3C). $\mathrm{NH}_{4} \mathrm{Cl}^{-}$experiments revealed that the inhibitory effects of $\mathrm{BA}$ and $\mathrm{EtOH}+\mathrm{FA}$ on $\mathrm{Cl} / \mathrm{HCO}_{3}{ }^{-}$exchangers $\left(\mathrm{HCO}_{3}{ }^{-}\right.$efflux $)$and on $\mathrm{Na} / \mathrm{HCO}_{3}{ }^{-}$co-transporters $\left(\mathrm{HCO}_{3}{ }^{-}\right.$ influx) were significantly reduced in the NIM811-treated groups compared to the controls, showing a protective effect of NIM811 on PDEC (Fig. 3D).

\section{NIM811 and CYA have no effects on pancreatic ductal fluid secretion}

Both in vivo and in vitro measurements revealed that NIM811 or CyA treatment can not prevent BA or EtOH+FA induced fluid secretiory damage in isolated ducts (Fig.4 A-D, E-F).

\section{NIM811 treatment protects mitochondrial function in acinar cells}

In vitro measurements of freshly isolated pancreatic acinar cells showed that NIM811 treatment decreased the BA- and EtOH-FA-induced loss of $\psi$ as effectively as we have seen in PDEC (Fig. 4A). However, results obtained from TOM20 staining suggest that NIM811 has no effect on mitochondrial mass in acinar cells (Fig. 5B). Microfluorometric measurements demonstrated that NIM811 alone has no toxic effects on acinar cells and has no effect on BA- or EtOH-FA-induced apoptosis, but is protective against BA- or EtOH-FAinduced necrosis (Fig. 5C).

NIM811 has therapeutic benefits in caerulein, taurolithocholic acid sulfate and ethanol and fatty acid induced $A P$

Firstly, we confirmed that per os administration of either 5 or $10 \mathrm{mg} / \mathrm{kg}$ NIM811 alone has no toxic effect on the pancreas (Fig 9.). Secondly, we tested the compound in three different experimental AP models, the caerulein (CER), alcohol and fatty acid (EtOH+FA) and the taurocholic (TAU)-induced ones (Niederau et al,1985; Huang et al, 2014; Perides et al,2010) . Importantly, both pretreatment 5 or $10 \mathrm{mg} / \mathrm{kg}$ NIM811 significantly reduced the elevation of serum amlylase activity, as well as pancreatic oedema, necrosis and leukoctye infiltration in experimental AP models (Figs. 6-8). In our study we also confirmed, that post treatment of $5 \mathrm{mg} / \mathrm{kg}$ or $10 \mathrm{mg} / \mathrm{kg}$ NIM811 has protective effects against pancreatic damage (Figs. 6-8.). 


\section{DISCUSSION}

Acute pancreatitis is a multifactorial disease (Hegyi and Petersen ,2013; Sahin-Toth and Hegyi, 2017 ) involving several types of cell, including acinar and ductal cells. None of the therapeutic efforts targeting only one of them have been successful. Intravenous administration of secretin, which targeted ductal cells only, was found either to be slightly beneficial or natural in AP (Renner et al,1983; Lankisch et al, 1983; Keim et al, 1985). On the other hand, neither gabexate mesilate nor trasylol, which effectively inhibit trypsin activity, had beneficial effects in AP (Imrie et al,1978; Buchler et al, 1993) (Imrie, Benjamin et al. 1978, Buchler, Malfertheiner et al. 1993). Therefore, we need to find common targets which can restore both acinar and ductal cell functions in AP.

Mitochondrial damage is one of the key pathophysiological events in the early phase of AP in both types of cell (Maleth et al, 2013; Hegyi and Petersen, 2013 ; Maleth and Hegyi,2016) It decreases ATP production, causing elevation of intracellular calcium concentration; moreover, it negatively influences ATP-dependent $\mathrm{Cl}^{-} \mathrm{HCO}_{3}^{-}$exchangers, CFTR $\mathrm{Cl}^{-}$channels in ductal cells and enzyme secretory processes in acinar cells (Maleth et al,2011; Maleth et al, 2013 ; Judak et al, 2014; Maleth et al,2015 ; Mukherjee et al,2016; Maleth and Hegyi,2016, Biczo and Vegh et al, 2018 ). In addition, mitochondrial damage is the main factor in determining cell death pathways necrosis and apoptosis. Release of mitochondrial cytochrome $\mathrm{c}$ into the cytosol causes apoptosis, whereas mitochondrial depolarization leads to necrosis (Odinokova et al, 2008) . Generally, the standard apoptotic pathway involves mitochondrial outer membrane permeabilization, which causes apoptotic factors like cytochrome c to be released from the inner membrane to the cytosol (Tait et al, 2010; Maleth et al, 2016 ). On the other hand, the opening of the mPTP leads to loss of $\psi$, ATP depletion, increased inner membrane permeability, mitochondrial swelling and necrotic cell death (Golstein et al, 2007; Halestrap et al, 2009; Maleth et al, 2016). Very uniquely, inhibition of mPTP could prevent both cell death mechanisms in PDEC, which is different from that seen in acinar cells, where only necrosis could have been prevented. All in all, inhibition of mPTP seems to be highly beneficial in both cell types. In the last decade, it has been proved that genetic or pharmacological inhibition of $\mathrm{mPTP}$ reduces BA- or EtOH+FAinduced AC damage as well as augmenting the severity of AP (Sah et al,2011; Mukherjee et al, 2016 ; Gukovskaya et al, 2016; Biczo and Vegh et al,2018). As regards ductal cells, we 
have shown earlier that both $\mathrm{BA}$ and $\mathrm{EtOH}+\mathrm{FA}$ induce inhibition of $\mathrm{HCO}_{3}{ }^{-}$secretion via severe mitochondrial damage in PDEC) (Maleth et al,. 2011, Maleth et al. 2015). Now, we have continued our experiments investigating the role of $\mathrm{mPTP}$ and its inhibition in this type of epithelial cell. First, we characterized the role of mPTP (both genetic and pharmacological CyA) inhibition in PDEC and found that its inhibition has a strong protective effect against the toxic effects of BA or EtOH+FA in ductal cells, suggesting that targeting mPTP may have general benefits. Although many mPTP inhibitors have been tested, none of them have been successful. CyA itself inhibits calcineurin, which leads to immunosuppressant activity and thus could negatively affect the treatment of patients due to hazardous infections. Clinical testing of non-immunosuppressive CyA derivatives Debio025 and TRO40303 was also stopped before reaching the "proof of concept" phase 2 clinical trials in AP because of its inconsistent behavior in other trials due to the facts noted in the introduction. Recently, other new mPTP inhibitors have been introduced in experimental studies. Isoxazoles had inconsistent effects in myocardial infarction (Sileikyte et al, 2016 ). Benzamides resulted in impaired ATP generation (Sileikyte et al, 2016; Javed et al, 2018) . Cinnamic anilides were shown to be effective in myocardial infarction (Fancelli et al, 2010); however, lately it has turned out that it has an age-related toxicity (Fang et al, 2019). Besides unsuccessful attempts, NIM811 seemed to be a perfect choice. It has been shown to be protective in several diseases, and until now no toxic effects have been demonstrated. Therefore, we continued our study by testing the effects of NIM811 on both ductal and acinar cells in vitro. We found that NIM811 reduces the mitochondrial damage caused by BA or EtOH+FA . Importantly, NIM811 decreased apoptosis levels during BA or EtOH+FA treatment in ductal cells, but not in acinar cells, a result which could be due to the observation that ductal cells have more mitochondria than acinar cells (Maleth et al, 2013). Surprisingly, inhibition of mPTP protected pancreatic ductal bicarbonate but fluid secretion during BA or EtOH+FA treatment. These data suggest that rescuing intracellular ATP level and the activity of $\mathrm{Na}+\mathrm{K}+-$ ATPase do not result in overall protection alone and other fluid transport mechanisms such as aquaporins may remain diminished (Venglovecz et al, 2018). Per os administration of 5 or $10 \mathrm{mg} / \mathrm{kg}$ NIM811 treatment alone had no toxic effect, but significantly reduced the severity of AP. We found that NIM811 treatment was more beneficial in the TAU than the EtOH+FA induced AP model. One of the explanations could be that besides the direct toxic effect of EtOH and FA, the non-oxidative metabolites of FA 
namely FAEE has even higher toxicity on the mitochondria both in acinar and ductal cells (Criddle et al, 2006; Petersen et al, 2009).

Taken together, mitochondrial function and bioenergetics play a crucial role in the development of AP; however, translation of the results to patient benefit is still missing (Maleth et al,2013 ;Mukherjee et al,2016 ;Maleth and Hegyi,2016 ; Gukovskaya et al, 2016; Biczo and Vegh et al,2018). In this study, we were the first to confirm that the mPTP inhibitor NIM811 is a highly suitable compound to be tested in clinical trials. As a next step, the companies should organize phase 2 clinical trials with the use of this novel and promising drug candidate. 
Mitochondrial membrane potencial measurements revealed that genetic inhibition of mPTP significantly reduces the mitochondrial membrane potencial loss compared to WT controls during the administration of bile acid $(500 \mu \mathrm{m}$ CDC) or ethanol $(100 \mathrm{mM})$ and fatty acid $(200 \mu \mathrm{M}$ FA) treatment (Fig1. A) ( WT control vs WT BA ***p $<0.001$,WT BA vs Cyp D KO BA ${ }^{* *} p<0.002$, WT control vs Cyp D KO BA p=07.12,WT control vs. WT EtOH+FA $p<0.01$, WT EtOH+FA vs KO ETOH+FA * p<0.05, WT control vs Cyp D KO EtOH+FA p=0.145) $\mathrm{n}=4-6$ experiments/group, data means \pm SEM. Results from the immunostainings revealed a significant decrease of the TOM20 stainings in BA; EtOH+PA or CCCP treated WT ducts , results were compared to Cyp D KO stainings . (Fig1.B) $\left({ }^{*} \mathrm{p}<0.05\right)$. Genetic inhibition of mPTP also decreased the necrosis and apoptosis levels during bile acid; ethanol or fatty acid $\begin{array}{llll}\text { or } & \mathrm{CCCP} & \text { treatment } & (* \mathrm{p}<0.05)\end{array}$ Representative traces from the pancreatic ductal $\mathrm{HCO}_{3}$ - secretion measurements (Fig.1.D) Our data revealed that recovery from the alkalosis grades were significantly lower due to BA or ETOH+FA administration $(* \mathrm{p}<0.05)$ compared to the results from Cyp D KO ducts (Fig1.E). Recovery from the acidosis grades were significantly lower in the WT ducts due to the treatment with BA or EtOH and FA $\left({ }^{*} p<0.05\right)$, while in Cyp D KO ducts these grades were significantly higher $\left({ }^{*} \mathrm{p}<0.05\right) . \mathrm{n}=5-7$ experiments/group, data means \pm SEM. 
Figure2. CYA reduces the severity of bile acid or ethanol and fatty acid induced pancreatic ductal damage
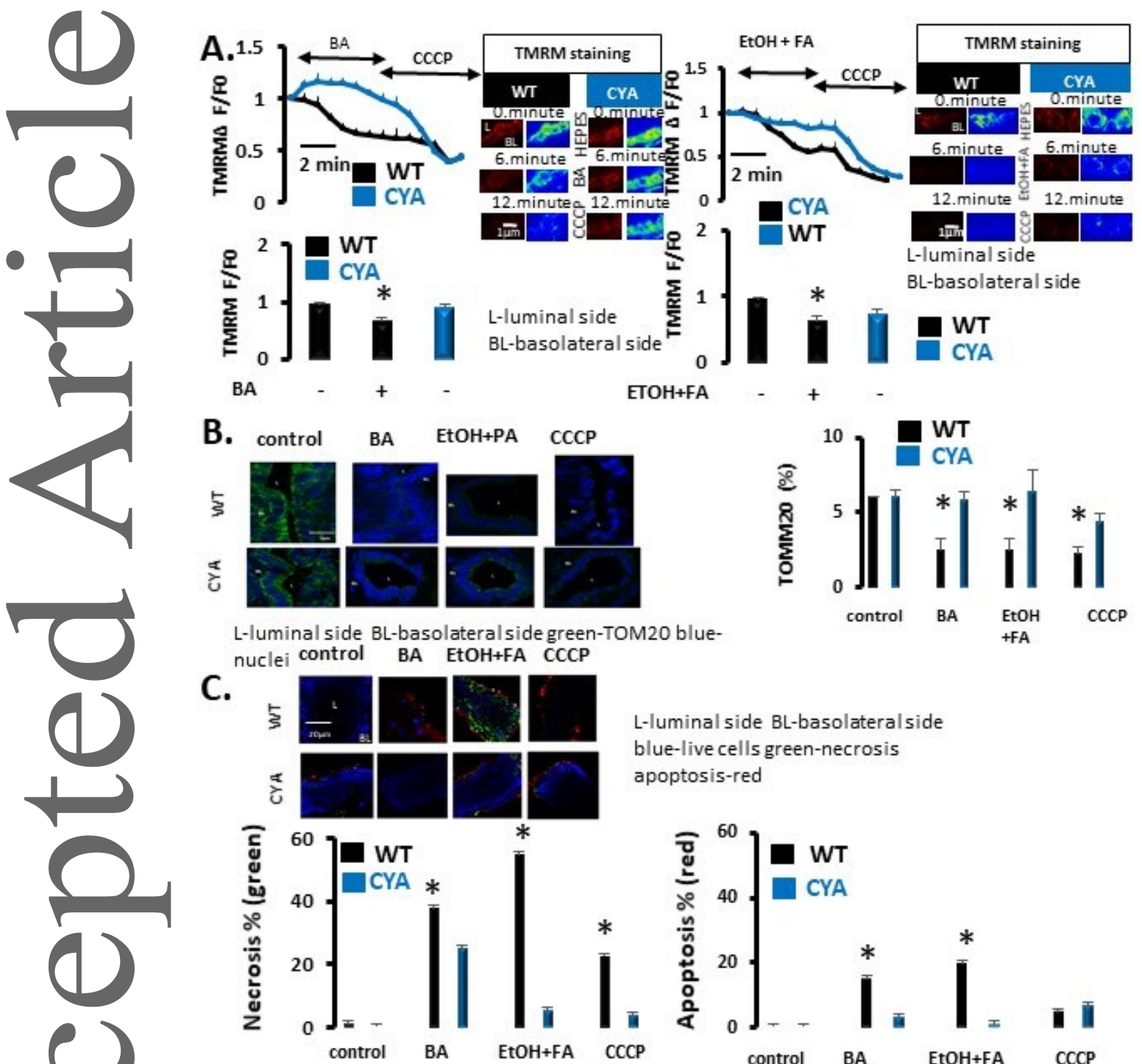

L-luminal side BL-basolateralside blue-live cells green-necrosis apoptosis-red
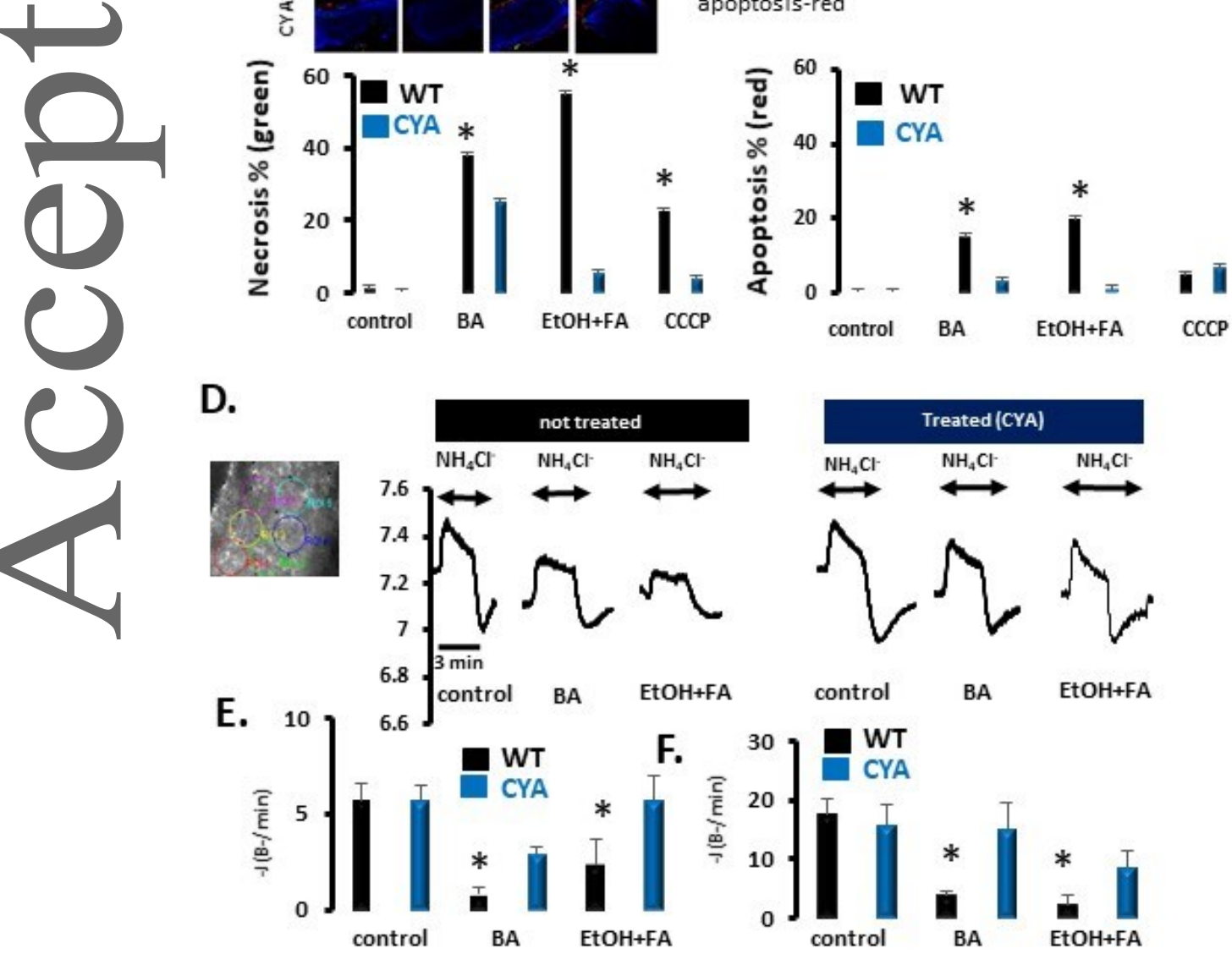

This article is protected by copyright. All rights reserved. 
$2 \mu \mathrm{M}$ CYA treatment reduced the drop of mitochondrial membrane potencial loss which accured due to the BA or ETOH+FA treatment. (WT vs. CYA) (Fig.2.A). In WT ducts BA or $\mathrm{ETOH}+\mathrm{FA}$ treatment resulted in significantly reduced mitochondrial membrane potencial (WT control vs WT BA *p<0.05, WT control vs WT EtOH +FA p<0.05), while between WT control groups compared to CYA treated BA or EtOH+FA there were no significant decrease. TOM20 levels were significantly reduced in BA; ETOH+FA or CCCP control (not CYA treated ) ducts, while in the CYA treated groups the percentage of TOM20 stained area were significantly higher $($ Fig2.B) $* p<0.05$. Between the control groups (WT control or only CYA treated samples) we found no significant alterations in the stainings. Necrosis levels were intensively elevated in BA or EtOH treated groups in WT ducts but not in CYA treated groups (Fig.2.C). Apoptosis levels were significantly higher as well in the not CYA treated groups compared to the CYA treated groups (Fig2. C).

Measurements of $\mathrm{HCO}_{3}{ }^{-}$secretion levels revealed a significant difference in WT and CYA treated ducts during the administration of BA ( $<<0.05$ WT BA vs CYA BA) or EtOH+FA $\left({ }^{*} \mathrm{p}<0.05\right)$. In WT ducts the levels of base flux $(-\mathrm{J}(\mathrm{B}-/ \mathrm{min})$ grades were significantly decreased (Fig2.E,F) due to BA (WT vs WT BA p<0.05) or ETOH+FA (WT vs WT $\mathrm{EtOH}+\mathrm{PA} \mathrm{p}<0.05)$ treatment $(\mathrm{Fig} 2 \mathrm{E}, \mathrm{F})$. Recovery from alkalosis (Figure 2. E) and recovery from acidosis values are presented in base flux $((-\mathrm{J}(\mathrm{B}-/ \mathrm{min})$ grades respectively, with $\pm \mathrm{SEM}$. Comparison within CYA treated groups revealed no significant difference (CYA control vs CYA BA p=0.644). 
Figure3. NIM811 protects mitochondrial and cell function in PDEC

(1)

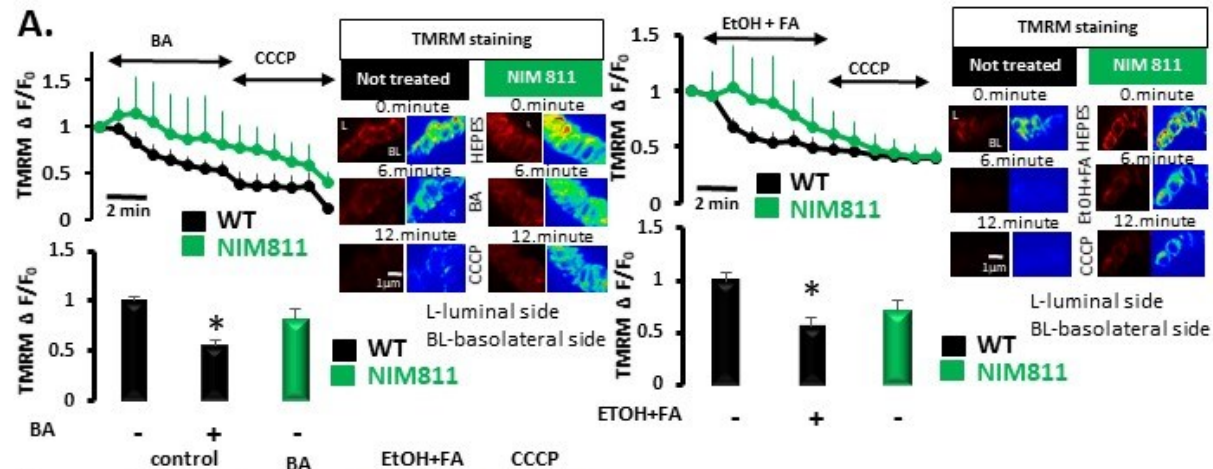

B.

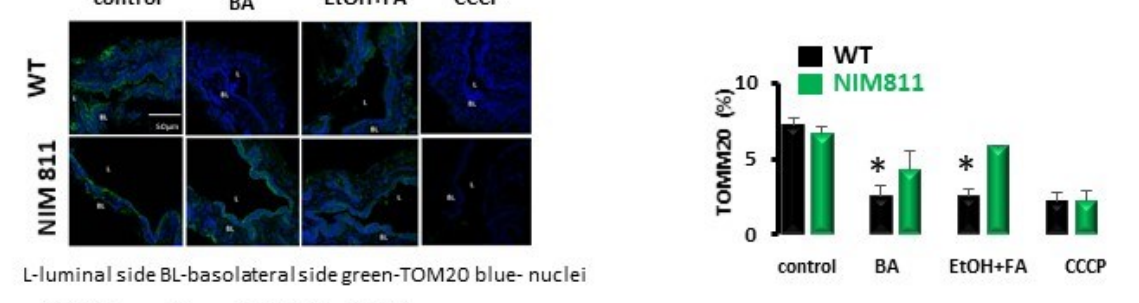

C. control BA EtOH+FA CCCP
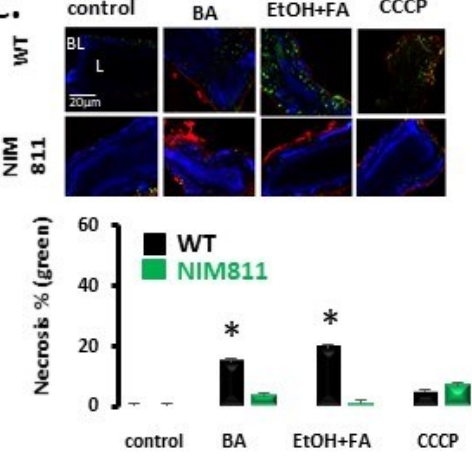

L-luminal side, BL-basolateral side, bluelive cells, green-necrosis, apoptosis-red

10 )
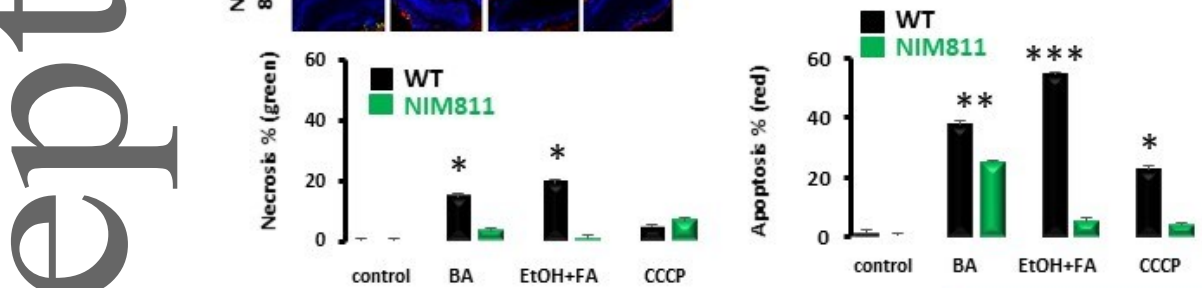

D.
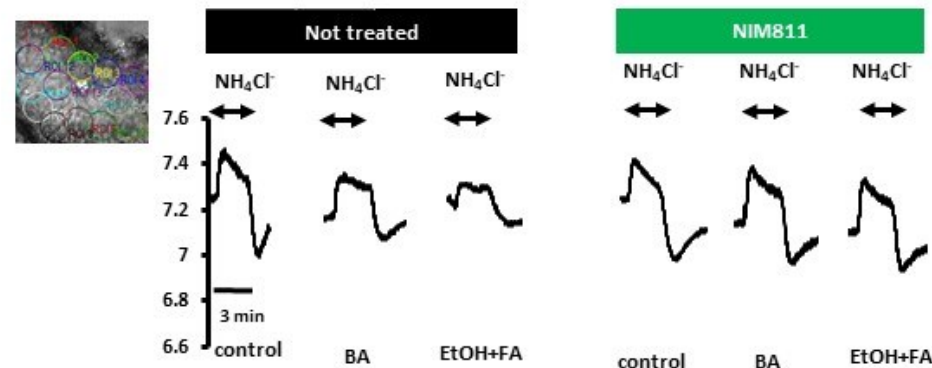

C
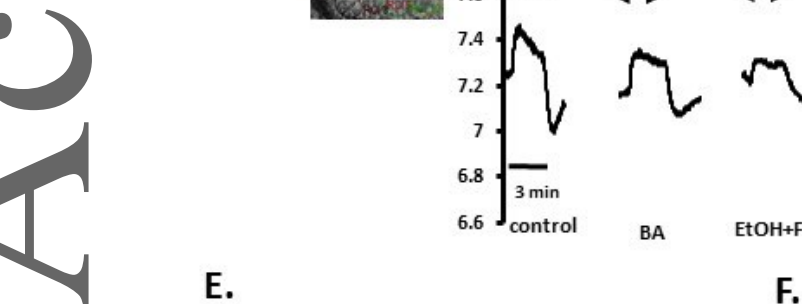

E.

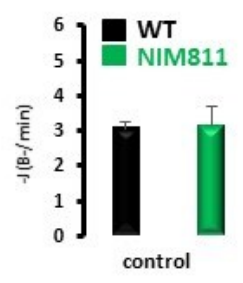

F.

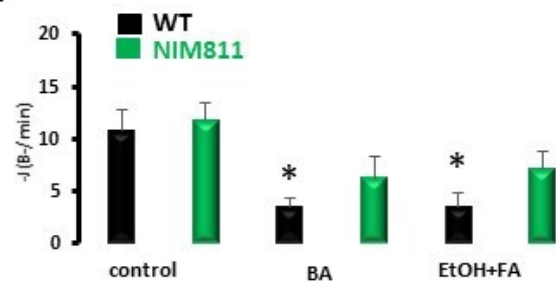

This article is protected by copyright. All rights reserved. 
NIM811 treated ducts revealed a significantly consolidated loss of mitochondrial membrane potencial during the BA (WT BA vs NIM811 BA *p<0.05) or ETOH+FA (WT ETOH+FA vs NIM811 ETOH+FA *p<0.05) treatment (Fig.3A) . In NIM811 treated ducts the percentage of fluorescence intensity were significantly higher compared to not NIM811 treated ducts during $\mathrm{BA}$ or $\mathrm{ETOH}+\mathrm{FA}$ administration. In $\mathrm{CCCP}$ treated ducts we found no significant difference in the amount of TOM20 stainings in the aspect of NIM811 treated or not treated groups. NIM811 itself did not alter the value of TOM20 stainings compared to the WT control samples (Fig.3B).

NIM811 decreased the numbers of apoptotic and necrotic cells during bile acid or ethanol and fatty acid treatment (Fig.3C) (WT BA vs NIM811 BA *p<0.05, WT EtOH+FA vs NIM811 $\left.{ }^{*} \mathrm{p}<0.05\right)$. While during the administration of CCCP the apoptosis and necrosis grades were not significantly different in the comparative groups (Fig.3.C).

NIM811 treatment did not decreased the $\mathrm{HCO}_{3}{ }^{-}$secretion grades (control, Fig.3 D,E,F), while during the adminsitration of BA or ETOH+FA treatment it had a protective effect against the reduction of $\mathrm{HCO}_{3}{ }^{-}$secretory levels (Fig.3E/F) (WT BA vs NIM811 BA *p $<0.05$, WT EtOH+FA vs NIM811 EtOH+FA *p<0.05). In the aspect of recovery levels from alkali load during EtOH and FA treatment, the difference were not sigfnificant in WT EtOH+FA compared to the NIM811 and EtOH+FA treated groups (Fig.3E). 
Figure 4. Pancreatic ductal fluid secretion is not altered by NIM811 or CYA treatment

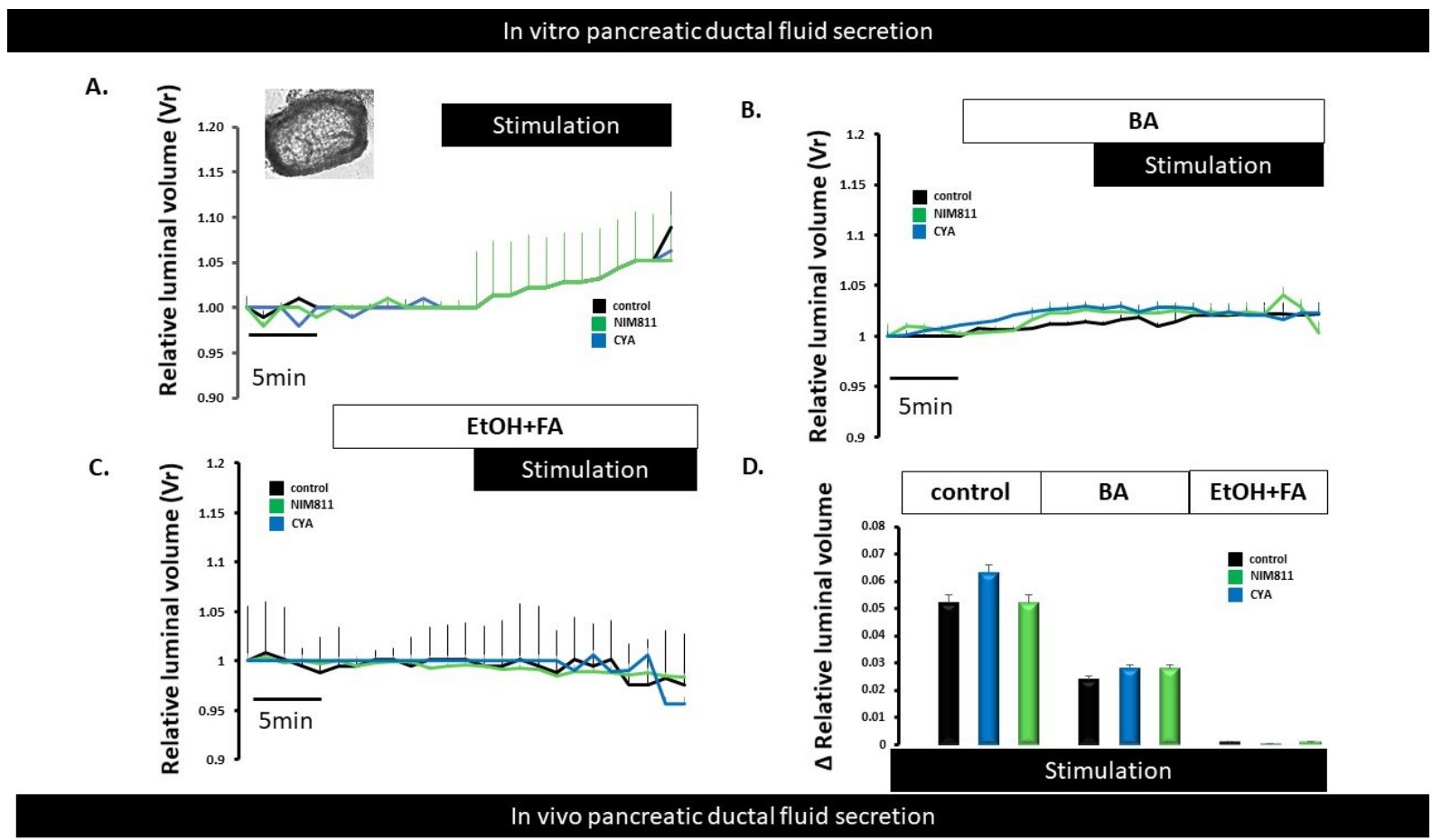

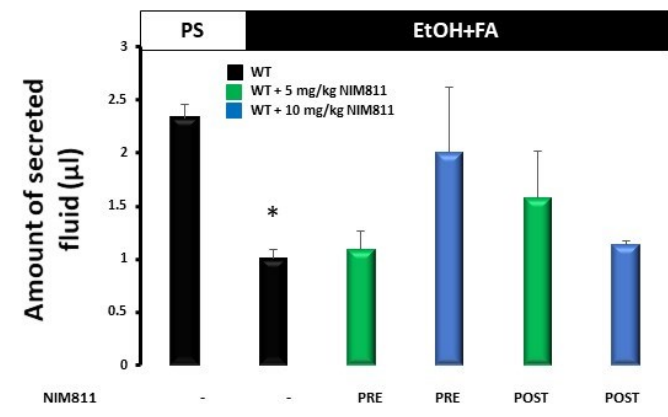

F.

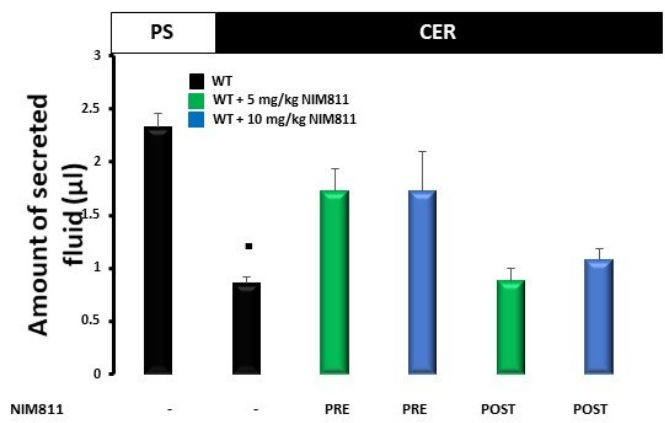

In vitro fluid secretion was stimulated by $5 \mu \mathrm{M}$ forskolin and $100 \mu \mathrm{M}$ IBMX (stimulation). BA or EtOH+PA treatment inhibited the luminal swelling (Fig.4.B-C). Figure 4D represents the relative luminal volume changes during forskolin and IBMX stimulation (Figure4.D). Means \pm SEM. $\mathrm{n}=5$-10 ducts/group. In vivo fluid secretion measurements were performed after the induction of CER or EtOH+FA induced AP (Fig.4.E-F.). These experiments confirmed that pancreatic ductal fluid secretion is not affected by NIM811 or CyA. (Fig.4.E-F). ${ }^{*} p<0.05$ WT PS vs. WT EtOH+FA, $" \mathrm{p}<0.05$ WT PS vs. WT CER $n=4-7$ animal/group 
Figure5. NIM811 treatment protects mitochondrial function in pancreatic acinar cells

A.
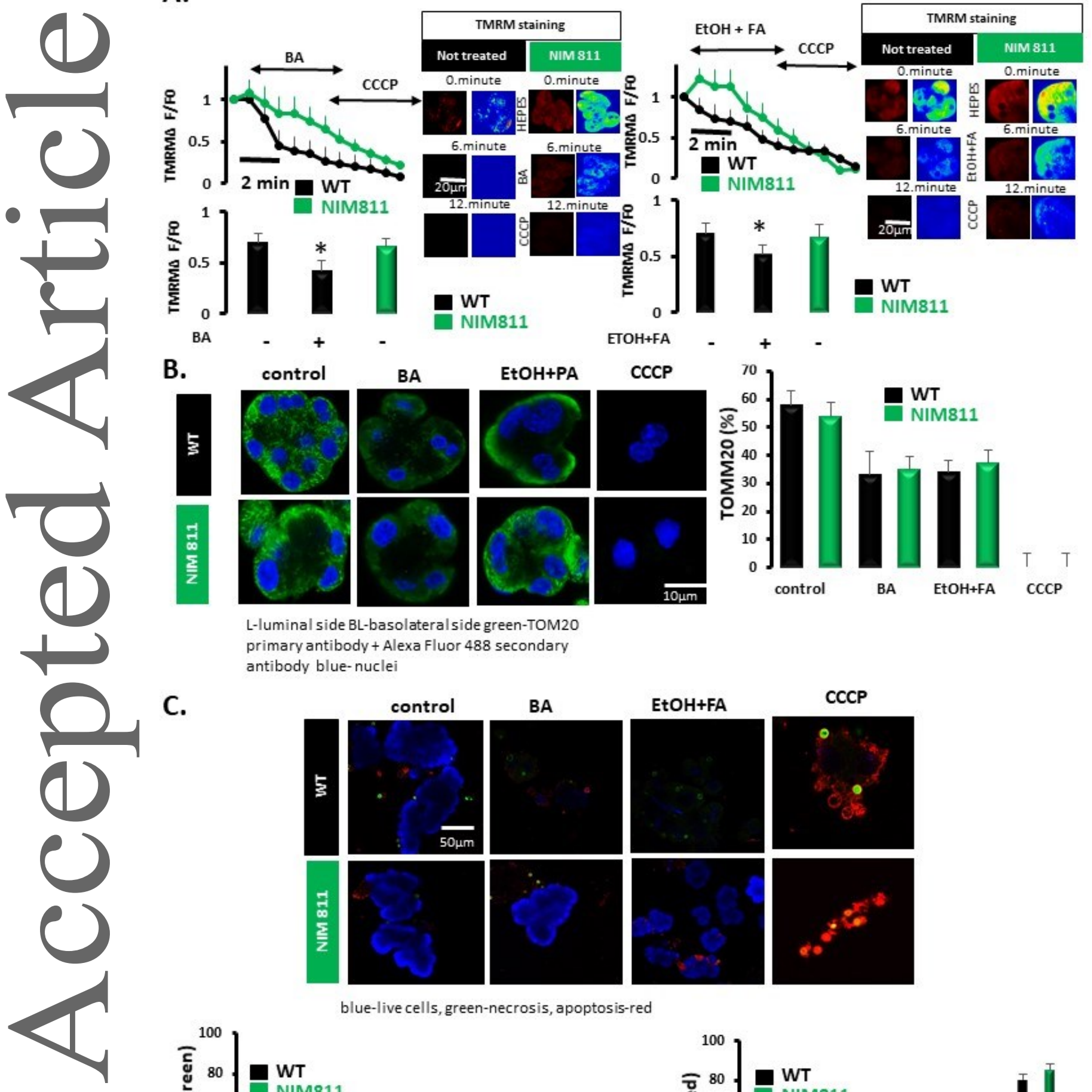

B.
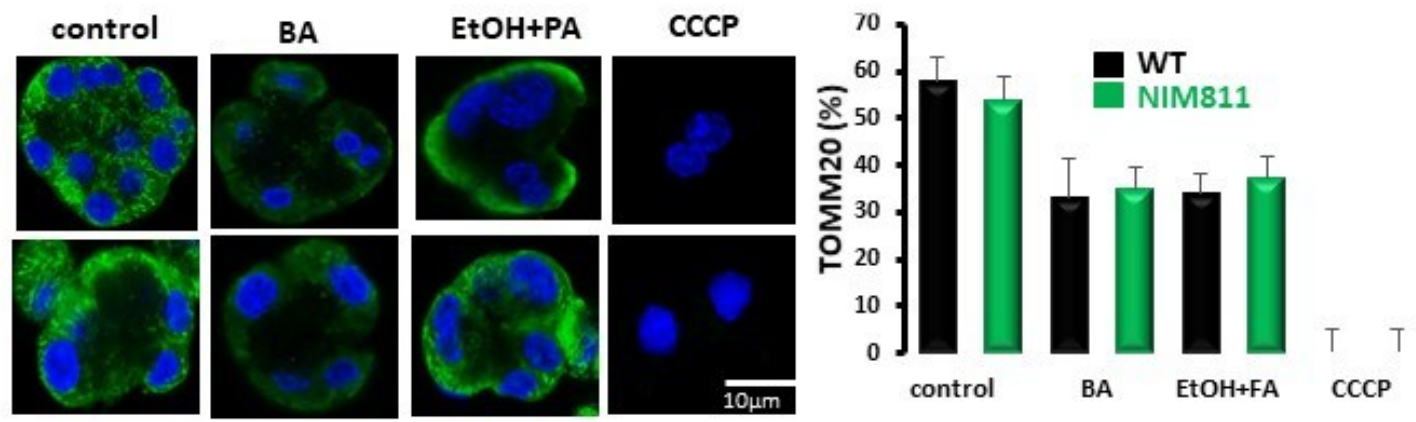

L-luminal side BL-basolateral side green-TOM20

primary antibody + Alexa Fluor 488 secondary antibody blue-nuclei

C.

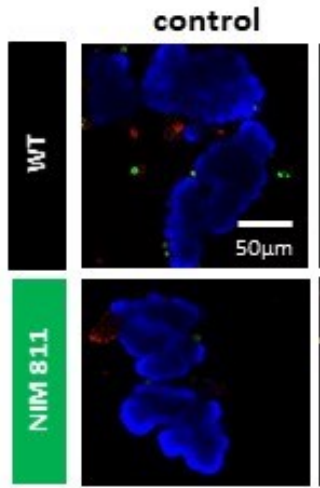

BA

EtOH+FA

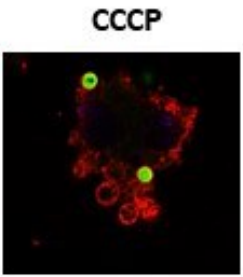

blue-live cells, green-necrosis, apoptos is-red
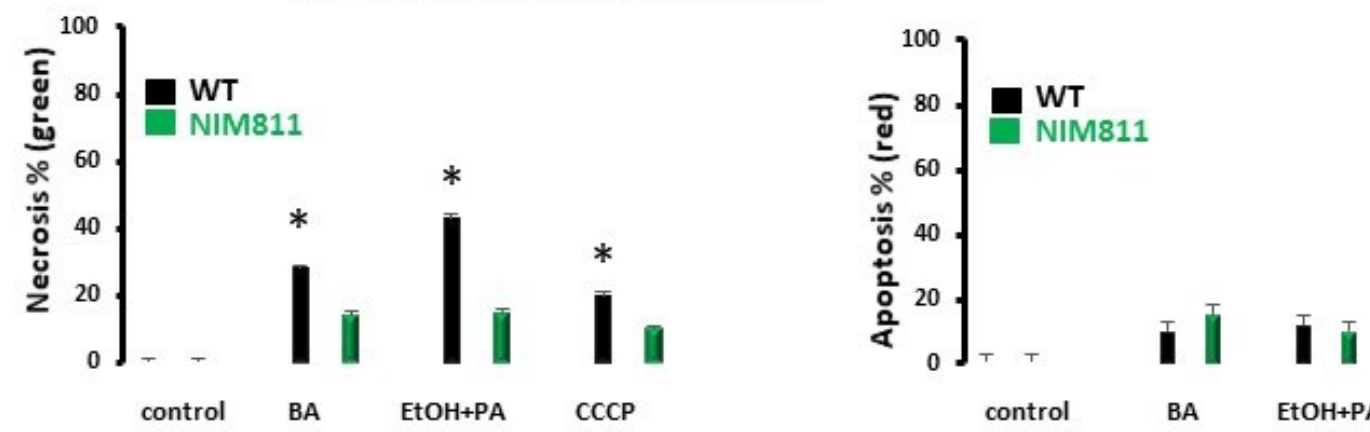

control

BA EtOH+PA

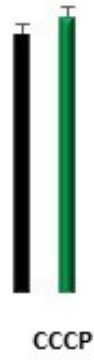

This article is protected by copyright. All rights reserved. 
Mitochondrial membrane potencial measurements revealed a significant difference between WT not NIM811 treated and the NIM811 treated acinar cell response due to bile acid or ethanol and fatty acid treatment (Fig.5A) (WT BA vs NIM811 BA*p<0.05; WT EtOH+FA vs NIm811 ETOH+FA * $\mathrm{p}<0.05)$. Significant difference was detected between the NIM811 treated acinar cells and the groups which were not treated with NIM811 (Fig.5A) during BA or $\mathrm{ETOH}+\mathrm{FA}$ treatment. Mitochondrial protein TOM20 levels did not show difference in the NIM811 treated or not treated groups after BA, ETOH+FA or CCCP treatment (Fig.5B) $(\mathrm{p}>0.05)$. In necrosis levels we found significant difference between NIM811 treated and not treated groups in BA or ETOH+FA (Fig.5C) $\left({ }^{*} \mathrm{p}<0.05\right)$. However, in CCCP treated groups we found no difference (Fig.5C). Apoptosis levels were not altered significantly by NIM811 during $\mathrm{BA}$ or $\mathrm{ETOH}+\mathrm{FA}$ treatment. 
vs CER + pre 10mg/kg NIM811 ). In the aspect of CER induced pancreatitis both $5 \mathrm{mg} / \mathrm{bwkg}$ NIM811 (Fig.6 A-F, p<0.05 WT CER vs. pre 5mg/bwkg NIM811 CER) and pre $10 \mathrm{mg} / \mathrm{bwkg}$ NIM811 (Fig.6 A-F, p<0.05 WT CER vs. Pre 10mg/bwkg NIM811 CER)) treatment reduced the CER-induced damage. Post $5 \mathrm{mg} / \mathrm{kg}$ NIM811 treatment significantly reduced serum amylase levels compared to WT CER $\cdot \mathrm{p}<0.05, \cdot \cdots \mathrm{p}<0.001$ WT PS vs WT CER (Fig.6G-E). Post insult administration of $10 \mathrm{mg} / \mathrm{kg}$ NIM811 significantly reduced oedema and leukocyte infiltration levels compared to WT CER treated groups $\cdot-\mathrm{p}<0.05$ (Fig.6H), n=8-10 animals per group, data means \pm SEM). 
We performed TAU induced pancreatitis(Fig.7A-K), serum amylase measurements revealed that due to retrogrode infusion of TAU elevated serum amylase levels occured $(* * * p<0.01$ WT PS vs WT TAU Fig.6.B, -"-p<0.001 WT PS vs WT TAU Fig.7G) howewer 5 mg/bwkg or $10 \mathrm{mg} / \mathrm{bwkg}$ NIM811 treatment significantly reduced the enzyme levels both in the pre and post treatment (Fig. 7B **p $<0.02$ WT TAU vs pre $5 \mathrm{mg} / \mathrm{kg}$ NIM811+TAU, ${ }^{* *} \mathrm{p}<0.02$ WT TAU vs pre $10 \mathrm{mg} / \mathrm{kg}$ NIM811+TAU, , $\cdot-\mathrm{p}<0.001$ WT TAU vs. post 5mg/kg NIM811 TAU, $\cdots p<0.001$ WT TAU vs post 10mg/kg NIM811 +TAU ) the serum amylase levels were reduced compared to WT TAU treated groups (Fig.7B. and $7 \mathrm{G} * \mathrm{p}<0.01$ WT TAU vs. WT 5mg/bwkg NIM811 TAU and *p<0.01 WT TAU vs WT 10 mg/bwkg NIM811 TAU) . During pre NIM811 treatment oedema, necrosis and leukocyte infiltration scores were significantly decreased compared to the only TAU treated groups (Fig.7A,C,D,E p $<0.05$ WT TAU vs pre 5mg/bwkg NIM811 TAU/10mg/bwkg NIM811 TAU). Post insult administration of NIM811 decreased oedema, leukocyte infiltration and necrosis levels in the TAU group $(\cdots p<0.001$ Fig.7G-K) n=4-6 animals per group, data means \pm SEM). 
Figure8. NIM811 has protective effect against EtOH+FA induced pancreatic damage

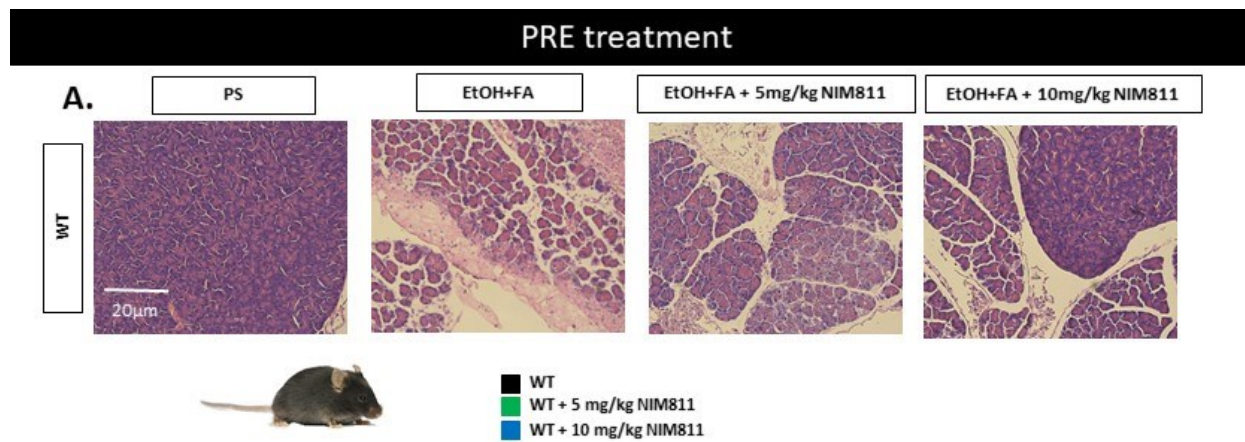

B.

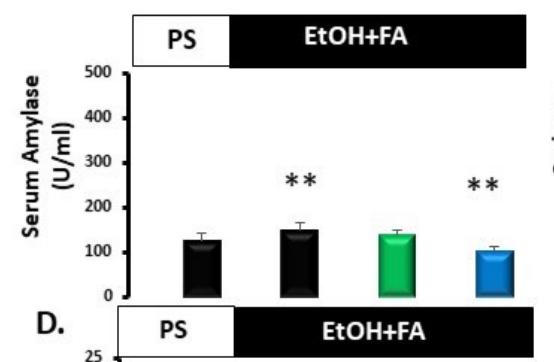

D.

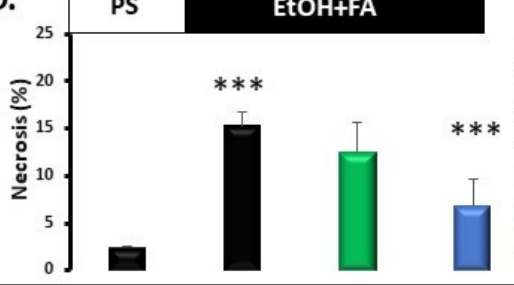

C.
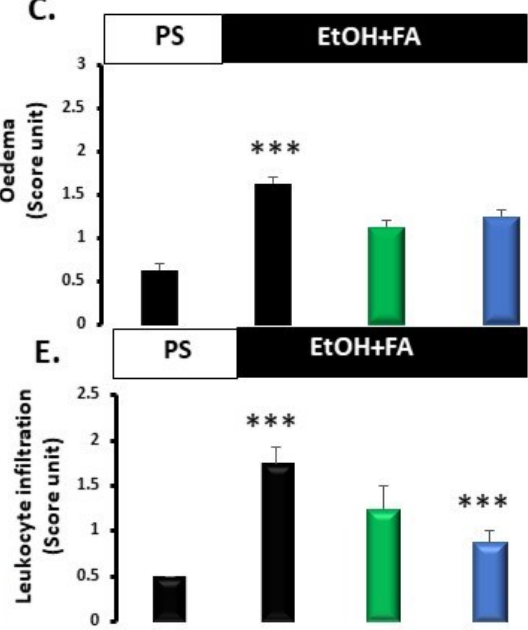

POST treatment
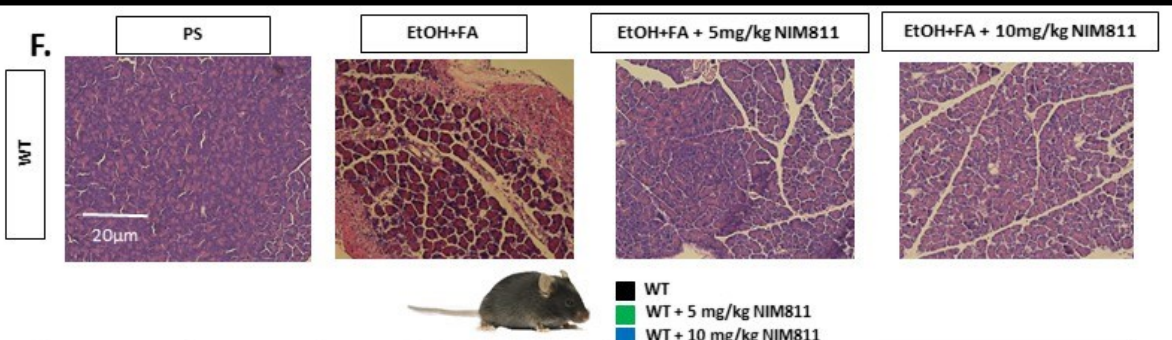

G.

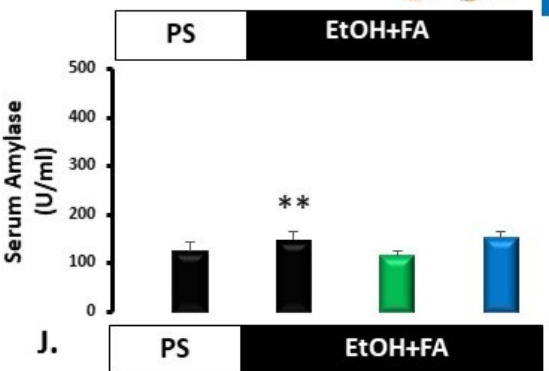

WT
WT $+5 \mathrm{mg} / \mathrm{kg}$ NIM811

$W T+5$ mg/kg NIM811
$W T+10 \mathrm{mg} / \mathrm{kg}$ NIM811

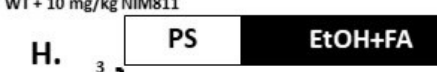

C
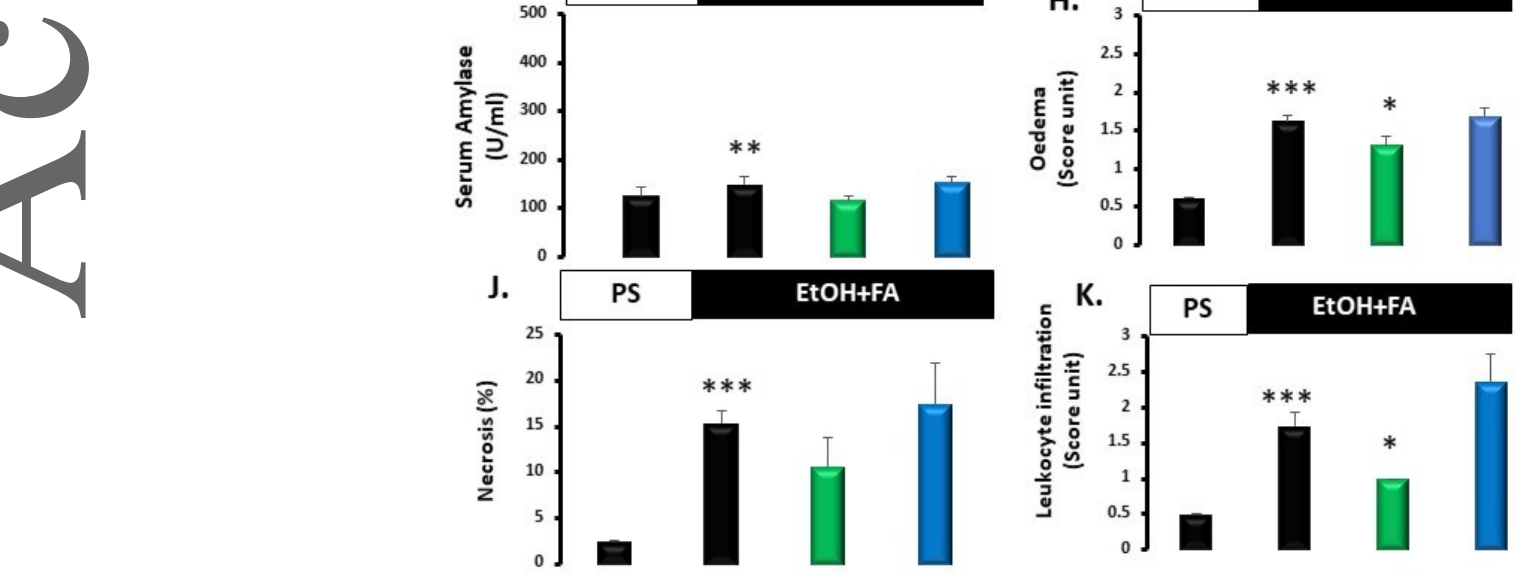

We performed EtOH+FA induced pancreatitis (Fig.8A-K). Serum amylase measurements revealed that in pre treatment of $10 \mathrm{mg} / \mathrm{kg}$ NIM811 significantly reduced serum amylase

This article is protected by copyright. All rights reserved. 
levels $* * \mathrm{p}<0.002 \mathrm{WT}$ EtOH+FA vs pre $10 \mathrm{mg} / \mathrm{kg}$ NIM811 +EtOH+FA (Fig.8B), ${ }^{*}$ p $<0.002$ WT PS vs Wt EtOH+FA (Fig.8B and G), in post NIM811 treatment serum amylase levels did not differ significantly compared to its ETOH+FA control (Fig.8G). In pre 10mg/kg NIM811 treatment leukocyte infiltration $(* * * \mathrm{p}<0.001 \mathrm{WT}$ EtOH+FA vs $10 \mathrm{mg} / \mathrm{kg}$ NIM811) and necrosis levels $(* * * \mathrm{p}<0.001 \mathrm{Wt} \mathrm{EtOH}+\mathrm{FA}$ vs $10 \mathrm{mg} / \mathrm{kg}$ NIM811) were significantly reduced compared to EtOH+FA AP group (Fig.8D-E). ${ }^{* *} \mathrm{p}<0.001$ WT PS vs Wt EtOH+FA in Fig.8C-E. Oedema and leukocyte infiltration levels were significantly reduced in post $5 \mathrm{mg} / \mathrm{kg}$ NIM811 treated groups compared to WT EtOH+FA groups $(* p<0.05$ WT EtOH+FA vs post 5 mg/kg NIM811 ) (Fig.8H and K) n=4-7 animals per group, data means \pm SEM).

Figure9. NIM811 itself does not induce pancreatic damage
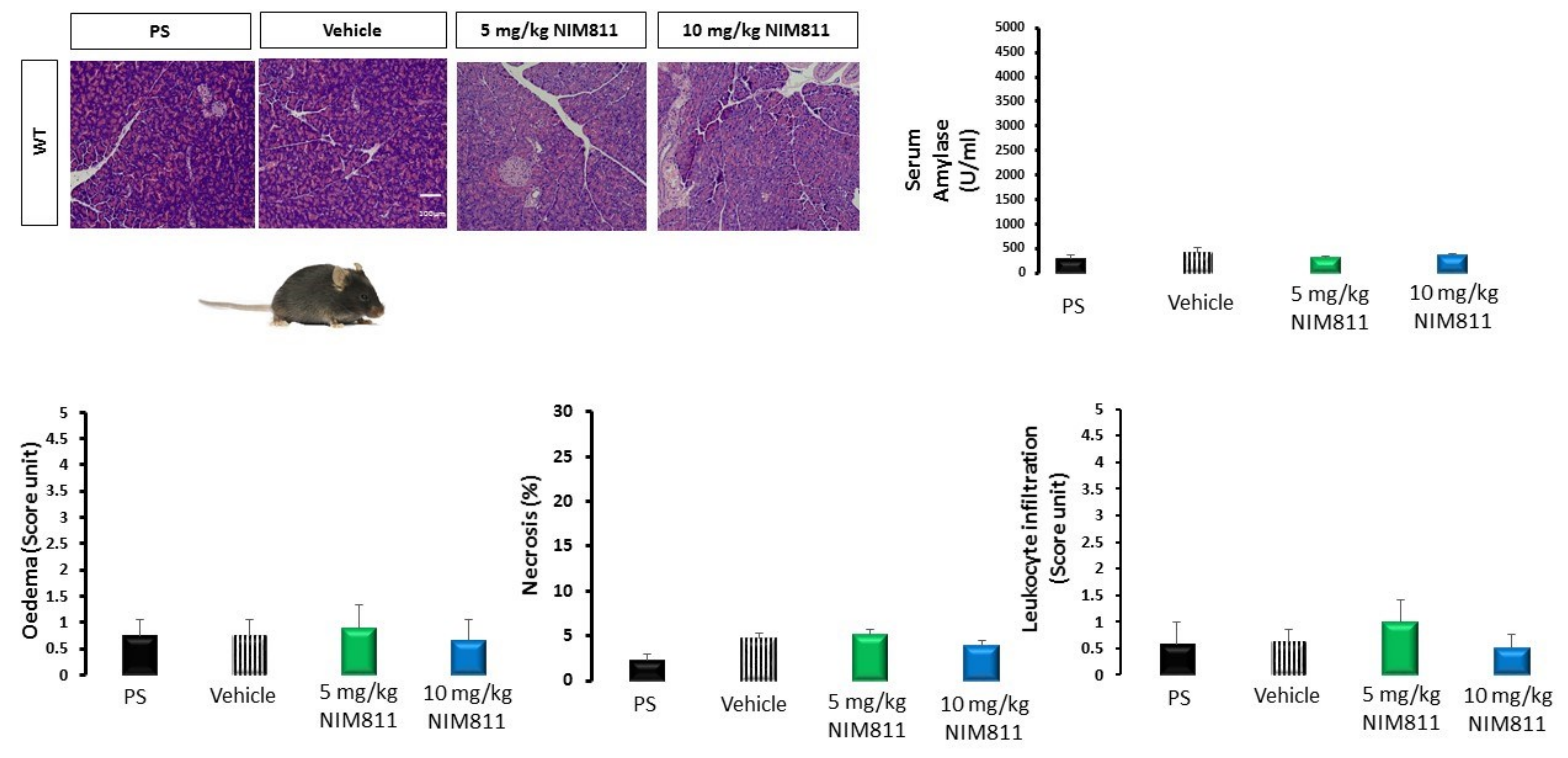

No significant difference was found between the NIM811-treated - (8.3\% Polyoxyl 40 hydrogenated castor oil, $8.3 \% \mathrm{EtOH}$ ) vs. the control groups. $\mathrm{n}=4-5$ animal/group 
Table1. Solutions used in our study

\begin{tabular}{|c|c|c|c|c|c|}
\hline & $\begin{array}{c}\text { HEPES } \\
\text { (Standard) } \mathrm{mM}\end{array}$ & $\begin{array}{c}\mathbf{H C O}_{3^{-}} \\
\text {(Standard) } \mathrm{mM}\end{array}$ & $\begin{array}{c}\mathrm{NH}_{4} \mathrm{Cl}^{-} \mathrm{HCO}_{3}- \\
\mathrm{mM}\end{array}$ & 1xTBS mM & $\begin{array}{c}\text { HBSS } \\
\text { (Standard) } \mathrm{mM}\end{array}$ \\
\hline $\mathrm{NaCl}$ & 140 & 115 & 95 & 150 & 0.137 \\
\hline $\mathrm{KCl}$ & 5 & 5 & 5 & - & 5.4 \\
\hline $\mathrm{CaCl}_{2}$ & 1 & 1 & 1 & - & 0.3 \\
\hline $\mathbf{M g C l}_{2}$ & 1 & 1 & 1 & - & - \\
\hline Glucose & 10 & 10 & 10 & - & 6 \\
\hline HEPES & - & - & - & - & - \\
\hline $\mathrm{NaHCO}_{3^{-}}$ & - & 25 & 25 & - & 4.2 \\
\hline $\mathrm{NH}_{4} \mathrm{Cl}^{-}$ & - & - & 20 & - & - \\
\hline Trisma Base & - & - & - & 50 & - \\
\hline $\mathrm{Na}_{2} \mathrm{HPO}_{4}$ & - & - & - & - & 0.25 \\
\hline $\mathrm{KH}_{2} \mathrm{PO}_{4}$ & - & - & - & - & 0.44 \\
\hline $\mathrm{MgSO}_{4}$ & - & - & - & - & 1.03 \\
\hline
\end{tabular}


Table 2. Oligonucleotide primers used in genotyping

\begin{tabular}{|l|l|}
\hline \multicolumn{2}{|l|}{ Primers } \\
\cline { 2 - 3 } \\
\hline F-null2 & TTCTCACCAGTGCATAGGGCTCTG \\
\hline LoxP1f & AAACTTCTCAGTCAGCTGTTGCCTCTG \\
\hline
\end{tabular}




\section{ADDITIONAL INFORMATION SECTION COMPETING INTEREST}

The authors have no conflicts of interest to disclose.

\section{AUTHOR CONTRIBUTION}

PH had the original idea, initiated the study, obtained funding and supervised the experimental procedures. Most of the protocols were designed by ET, JM, JF, VV, PP, ZR and PH. ET, NZ, AG and RE performed the experiments. Experiments were performed at the Laboratory of Cell Physiology, First Department of Medicine, University of Szeged or Institute for Translational Medicine and First Department of Medicine, University of Pécs, Pécs, Hungary. ERB contributed to the quantification of the histological samples. LT and GH provided the $\mathrm{Ppif}^{-/-}$mice to us and were involved in the data interpretation. ET, NZ and PH evaluated the statistical analysis. JF, JM, PP, ERB and VV provided conceptual advice on the experimental protocols (JF: isolation procedure for pancreatic acinar cells; JM: confocal microscopy and study design; ERB: histological quantification; PP and VV: fluorescence microscopy). ET and PH wrote the paper. JM, NZ, JF, AG, RE, PP, LT, GH, ERB, ZR and VV reviewed and contributed to the manuscript. All the authors approved the final manuscript.

\section{FUNDING}

This study was funded by a Momentum Grant from the Hungarian Academy of Sciences (LP2014-10/2014 to PH) as well as Economic Development and Innovation Operational Programme Grants and Project Grants from the National Research, Development and Innovation Office (GINOP-2.3.2-15-2016-00015, EFOP-3.6.2-16-2017-00006, K116634 to PH, UNKP-19-3-SZTE-303 to ET, K109756 to VV and PD115974 to JM and K119938 to ZR).

\section{AUTHORS' TRANSLATIONAL PERSPECTIVE}

Acute pancreatitis (AP) is a severe disorder with high morbidity, mortality and no specific treatment. It is generally accepted, that one of the earliest events in the disease initiation is the mitochondrial dysfunction and ATP depletion. It has been shown that the pancreatitis- 
inducing factors namely ethanol, fatty acids and bile acids open the membrane transition pore (mPTP) channel, keeping the channel continuously opened resulting in mitochondrial depolarization, lower ATP synthesis and cell necrosis both in pancreatic acinar and ductal cells. In this study, we provided strong evidence that one of the MPTP inhibitors, namely NIM811 is highly effective in different experimental pancreatitis models. Since NIM811 had no side-effects and passed the important phase 1 stage in the clinical trial process, companies should organize phase 2 clinical trials with the use of this novel and promising drug candidate. 


\section{REFERENCES}

Abu-El-Haija, M., et al., Accelerating the Drug Delivery Pipeline for Acute and Chronic Pancreatitis: Summary of the Working Group on Drug Development and Trials in Acute Pancreatitis at the National Institute of Diabetes and Digestive and Kidney Diseases Workshop. Pancreas, 2018. 47(10): p. 1185-1192.

Arai, M., et al., Resistance to cyclosporin A derives from mutations in hepatitis $\mathrm{C}$ virus nonstructural proteins. Biochem Biophys Res Commun, 2014. 448(1): p. 56-62.

Argent, B.E., et al., Morphological, biochemical and secretory studies on rat pancreatic ducts maintained in tissue culture. Q J Exp Physiol, 1986. 71(4): p. 633-48.

Atar, D., et al., Effect of intravenous TRO40303 as an adjunct to primary percutaneous coronary intervention for acute ST-elevation myocardial infarction: MITOCARE study results. Eur Heart J, 2015. 36(2): p. 112-9.

Baines, C.P., et al., Loss of cyclophilin D reveals a critical role for mitochondrial permeability transition in cell death. Nature, 2005. 434(7033): p. 658-62.

Balazs,A.,et al., Ductal Mucus Obstruction and Reduced Fluid Secretion Are Early Defects in Chronic Pancreatitis.Front Physiol.2018 May 29;9:632.

Biczo, G., Vegh, ET. et al., Mitochondrial Dysfunction, Through Impaired Autophagy, Leads to Endoplasmic Reticulum Stress, Deregulated Lipid Metabolism, and Pancreatitis in Animal Models. Gastroenterology, 2018. 154(3): p. 689-703.

Buchler, M., et al., Gabexate mesilate in human acute pancreatitis. German Pancreatitis Study Group. Gastroenterology, 1993. 104(4): p. 1165-70.

Criddle, DN., et al., Fatty acid ethyl esters cause pancreatic calcium toxicity via inositol trisphosphate receptors and loss of ATP synthesis. Gastroenterology, 2006. 130(3): p: 781-93. Cung, T.T., et al., Cyclosporine before PCI in Patients with Acute Myocardial Infarction. N Engl J Med, 2015. 373(11): p. 1021-31.

Ding, S.P., J.C. Li, and C. Jin, A mouse model of severe acute pancreatitis induced with caerulein and lipopolysaccharide. World J Gastroenterol, 2003. 9(3): p. 584-9. 
Fagenholz, P.J., et al., Increasing United States hospital admissions for acute pancreatitis, 1988-2003. Ann Epidemiol, 2007. 17(7): p. 491-7.

Fagenholz, P.J., et al., National study of United States emergency department visits for acute pancreatitis, 1993-2003. BMC Emerg Med, 2007. 7: p. 1.

Fancelli, D., et al., Cinnamic anilides as new mitochondrial permeability transition pore inhibitors endowed with ischemia-reperfusion injury protective effect in vivo. J Med Chem, 2014. 57(12): p. 5333-47.

Fang, J., et al., An Inhibitor of the Mitochondrial Permeability Transition Pore Lacks Therapeutic Efficacy Following Neonatal Hypoxia Ischemia in Mice. Neuroscience, 2019.

Fernandez-Salazar., et al., Basolateral anion transport mechanisms underlying fluid secretion by mouse, rat and guinea-pig pancreatic ducts. $J$ Physiol, 2004. 556(Pt 2), 415-428

Garbaisz, D., et al., Attenuation of skeletal muscle and renal injury to the lower limb following ischemia-reperfusion using mPTP inhibitor NIM-811. PLoS One, 2014. 9(6): p. e101067.

Golstein, P. and G. Kroemer, Cell death by necrosis: towards a molecular definition. Trends Biochem Sci, 2007. 32(1): p. 37-43.

Gout, J., et al., Isolation and culture of mouse primary pancreatic acinar cells. J Vis Exp, 2013(78).

Gukovskaya, A.S., S.J. Pandol, and I. Gukovsky, New insights into the pathways initiating and driving pancreatitis. Curr Opin Gastroenterol, 2016.

Halestrap, A.P., What is the mitochondrial permeability transition pore? J Mol Cell Cardiol, 2009. 46(6): p. 821-31.

Hegyi, P. and O.H. Petersen, The exocrine pancreas: the acinar-ductal tango in physiology and pathophysiology. Rev Physiol Biochem Pharmacol, 2013. 165: p. 1-30.

Hegyi, P., M.A. Gray, and B.E. Argent, Substance P inhibits bicarbonate secretion from guinea pig pancreatic ducts by modulating an anion exchanger. Am J Physiol Cell Physiol, 2003. 285(2): p. C268-76. 
Hegyi, P., et al., Measurement of intracellular pH in pancreatic duct cells: a new method for calibrating the fluorescence data. Pancreas,2004. 28(4):427-34.

Huang, W., Fatty acid ethyl ester synthase inhibition ameliorates ethanol-induced $\mathrm{Ca}^{2+}$ dependent mitochondrial dysfunction and acute pancreatitis. Gut 2014. 63, 1313-1324.

Huang, Z.L., et al., Cyclophilin inhibitor NIM811 ameliorates experimental allergic encephalomyelitis. J Neuroimmunol, 2017. 311: p. 40-48.

Imrie, C.W., et al., A single-centre double-blind trial of Trasylol therapy in primary acute pancreatitis. Br J Surg, 1978. 65(5): p. 337-41.

Javed, M.A., et al., TRO40303 Ameliorates Alcohol-Induced Pancreatitis Through Reduction of Fatty Acid Ethyl Ester-Induced Mitochondrial Injury and Necrotic Cell Death. Pancreas, 2018. 47(1): p. 18-24.

Judak, L., et al., Ethanol and its non-oxidative metabolites profoundly inhibit CFTR function in pancreatic epithelial cells which is prevented by ATP supplementation. Pflugers Arch, 2014. 466(3): p. 549-62.

Keim, V., et al., Failure of secretin to prevent or ameliorate cerulein-induced pancreatitis in the rat. Hepatogastroenterology, 1985. 32(2): p. 91-6.

Kui, B.,Balla Z. et al., New insights into the methodology of L-arginine-induced acute pancreatitis. PLoS One, 2015. 10(2): p. e0117588.

Lankisch, P.G., et al., Influence of secretin on the course of acute experimental pancreatitis in rats. Digestion, 1983. 26(4): p. 187-91.

Lawitz, E., et al., Safety, pharmacokinetics, and antiviral activity of the cyclophilin inhibitor NIM811 alone or in combination with pegylated interferon in $\mathrm{HCV}$-infected patients receiving 14 days of therapy. Antiviral Res, 2011. 89(3): p. 238-45.

Liu, Q., et al., Small-for-Size Liver Transplantation Increases Pulmonary Injury in Rats: Prevention by NIM811. HPB Surg, 2012. 2012: p. 270372.

Maleth, J. and P. Hegyi, Ca2+ toxicity and mitochondrial damage in acute pancreatitis: translational overview. Philos Trans R Soc Lond B Biol Sci, 2016. 371(1700). 
Maleth, J., et al., Alcohol disrupts levels and function of the cystic fibrosis transmembrane conductance regulator to promote development of pancreatitis. Gastroenterology, 2015. 148(2): p. 427-39 e16.

Maleth, J., et al., Central role of mitochondrial injury in the pathogenesis of acute pancreatitis. Acta Physiol (Oxf), 2013. 207(2): p. 226-35.

Maleth, J., et al., Non-conjugated chenodeoxycholate induces severe mitochondrial damage and inhibits bicarbonate transport in pancreatic duct cells. Gut, 2011. 60(1): p. 136-8.

Mukherjee, R., et al., Mechanism of mitochondrial permeability transition pore induction and damage in the pancreas: inhibition prevents acute pancreatitis by protecting production of ATP. Gut, 2016. 65(8): p. 1333-46.

Niederau, C., L.D. Ferrell, and J.H. Grendell, Caerulein-induced acute necrotizing pancreatitis in mice: protective effects of proglumide, benzotript, and secretin. Gastroenterology, 1985. 88(5 Pt 1): p. 1192-204.

Odinokova, I.V., et al., Mitochondrial mechanisms of death responses in pancreatitis. J Gastroenterol Hepatol, 2008. 23 Suppl 1: p. S25-30.

Pallagi, P., Balla Z, Singh AK et al., The role of pancreatic ductal secretion in protection against acute pancreatitis in mice*. Crit Care Med, 2014. 42(3): p. e177-88.

Parniczky, A., et al., Prospective, Multicentre, Nationwide Clinical Data from 600 Cases of Acute Pancreatitis. PLoS One, 2016. 11(10): p. e0165309.

Peery, A.F., et al., Burden of gastrointestinal disease in the United States: 2012 update. Gastroenterology, 2012. 143(5): p. 1179-1187 e3.

Perides, G., et al., Experimental acute biliary pancreatitis induced by retrograde infusion of bile acids into the mouse pancreatic duct. Nat Protoc, 2010. 5(2): p. 335-41.

Petersen, $\mathrm{OH} .$, et al., Fatty acids, alcohol and fatty acid ethyl esters: toxic Ca2+ signal generation and pancreatitis. Cell Calcium, 2009. 45(6): p. 634-42.

Piot, C., et al., Effect of cyclosporine on reperfusion injury in acute myocardial infarction. N Engl J Med, 2008. 359(5): p. 473-81. 
Readnower, R.D., et al., Post-injury administration of the mitochondrial permeability transition pore inhibitor, NIM811, is neuroprotective and improves cognition after traumatic brain injury in rats. J Neurotrauma, 2011. 28(9): p. 1845-53.

Rehman, H., et al., NIM811 prevents mitochondrial dysfunction, attenuates liver injury, and stimulates liver regeneration after massive hepatectomy. Transplantation, 2011. 91(4): p. 40612.

Renner, I.G., J.R. Wisner, Jr., and H. Rinderknecht, Protective effects of exogenous secretin on ceruletide-induced acute pancreatitis in the rat. J Clin Invest, 1983. 72(3): p. 1081-92.

Sah, R.P. and A. Saluja, Molecular mechanisms of pancreatic injury. Curr Opin Gastroenterol, 2011.27(5): p. 444-51.

Sahin-Toth, M. and P. Hegyi, Smoking and Drinking Synergize in Pancreatitis: Multiple Hits on Multiple Targets. Gastroenterology, 2017. 153(6): p. 1479-1481.

Shalbueva, N., et al., Effects of oxidative alcohol metabolism on the mitochondrial permeability transition pore and necrosis in a mouse model of alcoholic pancreatitis. Gastroenterology, 2013. 144(2): p. 437-446 e6.

Sileikyte, J. and M. Forte, Shutting down the pore: The search for small molecule inhibitors of the mitochondrial permeability transition. Biochim Biophys Acta, 2016. 1857(8): p. 11971202.

Stanciu, C., et al., Efficacy and safety of alisporivir for the treatment of hepatitis $\mathrm{C}$ infection. Expert Opin Pharmacother, 2019. 20(4): p. 379-384.

Tait, S.W. and D.R. Green, Mitochondria and cell death: outer membrane permeabilization and beyond. Nat Rev Mol Cell Biol, 2010. 11(9): p. 621-32.

Venglovecz, V., et al., The Importance of Aquaporin 1 in Pancreatitis and Its Relation to the CFTR Cl(-) Channel. Front Physiol, 2018. 9: p. 854.

Zeuzem, S., et al., Randomised clinical trial: alisporivir combined with peginterferon and ribavirin in treatment-naive patients with chronic HCV genotype 1 infection (ESSENTIAL II). Aliment Pharmacol Ther, 2015. 42(7): p. 829-44. 


\section{OPEN ACCESS}

Edited by: Alexei Tepikin,

University of Liverpool,

United Kingdom

Reviewed by:

Julia Gerasimenko,

Cardiff University, United Kingdom

Jason I. E. Bruce,

University of Manchester,

United Kingdom

*Correspondence:

Péter Hegyi

hegyi.peter@pte.hu

Specialty section: This article was submitted to

Gastrointestinal Sciences,

a section of the journal

Frontiers in Physiology

Received: 21 April 2018

Accepted: 15 June 2018

Published: 12 July 2018

Citation:

Venglovecz V, Pallagi P, Kemény $L V$,

Balázs A, Balla Z, Becskeházi E,

Gál E, Tóth E, Zvara Á, Puskás LG,

Borka K, Sendler M, Lerch MM,

Mayerle J, Kühn J-P, Rakonczay Z Jr. and Hegyi $P$ (2018) The Importance of Aquaporin 1 in Pancreatitis and Its

Relation to the CFTR $\mathrm{Cl}^{-}$Channel.

Front. Physiol. 9:854

doi: 10.3389/fphys.2018.00854

\section{The Importance of Aquaporin 1 in Pancreatitis and Its Relation to the CFTR $\mathrm{Cl}^{-}$Channel}

\author{
Viktória Venglovecz ${ }^{1}$, Petra Pallagi ${ }^{2}$, Lajos V. Kemény ${ }^{1}$, Anita Balázs ${ }^{2}$, Zsolt Balla ${ }^{3}$, \\ Eszter Becskeházi', Eleonóra Gál', Emese Tóth ${ }^{2}$, Ágnes Zvara ${ }^{4}$, László G. Puskás ${ }^{4}$, \\ Katalin Borka ${ }^{5}$, Matthias Sendler ${ }^{6}$, Markus M. Lerch ${ }^{6}$, Julia Mayerle ${ }^{6,7}$, \\ Jens-Peter Kühn ${ }^{8,9}$, Zoltán Rakonczay Jr. ${ }^{3}$ and Péter Hegyi',10,11*
}

${ }^{1}$ Department of Pharmacology and Pharmacotherapy, University of Szeged, Szeged, Hungary, ${ }^{2}$ First Department of Medicine, University of Szeged, Szeged, Hungary, ${ }^{3}$ Department of Pathophysiology, University of Szeged, Szeged, Hungary, ${ }^{4}$ Laboratory of Functional Genomics, Biological Research Centre, Hungarian Academy of Sciences, Szeged, Hungary, ${ }^{5}$ Second Department of Pathology, Semmelweis University, Budapest, Hungary, ${ }^{6}$ Department of Medicine A, University Medicine Greifswald, University of Greifswald, Greifswald, Germany, ${ }^{7}$ Department of Medicine II, Klinikum Grosshadern, Universitätsklinikum der Ludwig-Maximilians-Universität München, Munich, Germany, ${ }^{8}$ Institute of Radiology, University Medicine Greifswald, University of Greifswald, Greifswald, Germany, ${ }^{9}$ Institute and Policlinic of Radiology, University Hospital Carl Gustav Carus, TU Dresden, Dresden, Germany, ${ }^{10}$ MTA-SZTE Translational Gastroenterology Research Group, University of Szeged, Szeged, Hungary, ${ }^{11}$ Institute for Translational Medicine and First Department of Medicine, Medical School, University of Pécs, Pécs, Hungary

Aquaporins (AQPS) facilitate the transepithelial water flow involved in epithelial fluid secretion in numerous tissues; however, their function in the pancreas is less characterized. Acute pancreatitis (AP) is a serious disorder in which specific treatment is still not possible. Accumulating evidence indicate that decreased pancreatic ductal fluid secretion plays an essential role in AP; therefore, the aim of this study was to investigate the physiological and pathophysiological role of AQPs in the pancreas. Expression and localization of AQPs were investigated by real-time PCR and immunocytochemistry, whereas osmotic transmembrane water permeability was estimated by the dye dilution technique, in Capan-1 cells. The presence of AQP1 and CFTR in the mice and human pancreas were investigated by immunohistochemistry. Pancreatic ductal $\mathrm{HCO}_{3}{ }^{-}$and fluid secretion were studied on pancreatic ducts isolated from wild-type (WT) and AQP1 knock out (KO) mice using microfluorometry and videomicroscopy, respectively. In vivo pancreatic fluid secretion was estimated by magnetic resonance imaging. AP was induced by intraperitoneal injection of cerulein and disease severity was assessed by measuring biochemical and histological parameters. In the mice, the presence of AQP1 was detected throughout the whole plasma membrane of the ductal cells and its expression highly depends on the presence of $\mathrm{CFTR} \mathrm{Cl}^{-}$channel. In contrast, the expression of AQP1 is mainly localized to the apical membrane of ductal cells in the human pancreas. Bile acid treatment dose- and time-dependently decreased mRNA and protein expression of AQP1 and reduced expression of this channel was also demonstrated in patients suffering from acute and chronic pancreatitis. $\mathrm{HCO}_{3}{ }^{-}$and fluid secretion significantly decreased in AQP1 KO versus WT mice and the absence of 


\begin{abstract}
AQP1 also worsened the severity of pancreatitis. Our results suggest that AQP1 plays an essential role in pancreatic ductal fluid and $\mathrm{HCO}_{3}{ }^{-}$secretion and decreased expression of the channel alters fluid secretion which probably contribute to increased susceptibility of the pancreas to inflammation.
\end{abstract}

Keywords: pancreas, aquaporins, bile acids, $\mathrm{HCO}_{3}{ }^{-}$secretion, $\mathrm{CFTR} \mathrm{Cl}^{-}$channel

\section{INTRODUCTION}

The pancreas secretes 1.5-2 1 of fluid per day (Hegyi et al., 2011a; Pallagi et al., 2015). This fluid contains the inactive form of digestive enzymes and is prominently rich in $\mathrm{HCO}_{3}{ }^{-}$ produced by the ductal cells (Pallagi et al., 2015). The main functions of this $\mathrm{HCO}_{3}{ }^{-}$-rich fluid are (i) to prevent the premature activation of zymogens (Pallagi et al., 2011), (ii) to wash out the toxic factors (such as bile acids) from the ductal tree (Venglovecz et al., 2008), and (iii) to provide an alkaline environment in the duodenum for the optimal function of digestive enzymes (Steward et al., 2005). In the last few years, the importance of ion transport proteins has been highlighted in the course of pancreatitis; therefore intensive research has been conducted in order to characterize their pathological roles (Hegyi and Rakonczay, 2015; Pallagi et al., 2015). These clinical and experimental studies indicate that impaired ductal $\mathrm{HCO}_{3}{ }^{-}$ secretion makes the pancreas more susceptible to inflammatory diseases such as acute or chronic pancreatitis (CP) (Hegyi and Rakonczay, 2010; Hegyi et al., 2011b; Takacs et al., 2013; Pallagi et al., 2014; Maleth et al., 2015). In contrast, much less is known about the water transport processes, despite the fact that movement of electrolytes is osmotically coupled to water flow.

Aquaporins (AQPs) are small membrane proteins that primarily mediate the transport of water molecules and recent research also emphasize their importance in certain regulatory processes (Rodriguez et al., 2011; Ribatti et al., 2014; Madeira et al., 2015). In mammals, 13 AQP isoforms have been identified so far and a few of them show a species-specific expression pattern in the pancreas. In humans, AQP1, $-5,-8$, and -12 are present in the pancreas (Hurley et al., 2001; Tani et al., 2001; Furuya et al., 2002; Burghardt et al., 2003; Itoh et al., 2005). AQP1 is the first AQP which has been described and is exclusively permeable to water (Preston et al., 1992). The presence of this channel has been shown in the apical and lateral plasma membrane of centroacinar cells, and in the apical and basolateral membranes of intercalated and intralobular ducts (Furuya et al., 2002; Burghardt et al., 2003). AQP5 is an aquaglyceroporin which mediates the transport of glycerol, urea and other small solutes beside water. Expression of AQP5 has only been detected in the apical plasma membrane of intercalated ducts but not in the centroacinar cells (Burghardt et al., 2003). AQP1 and -5 are colocalized with the cystic fibrosis transmembrane conductance regulator (CFTR) $\mathrm{Cl}^{-}$channel at the apical membrane of the ductal cells which indicates that these channels influence each other's function (Burghardt et al., 2003). AQP8 and 12 are exclusively localized to centroacinar cells. AQP8 is expressed on the apical plasma membrane (Hurley et al., 2001;
Tani et al., 2001), whereas AQP12, a relatively new member of the AQP family, is an intracellular water channel (Itoh et al., 2005). Several studies suggest that altered expression or functions of AQPs are often associated with different diseases affecting the kidney, colon, lacrimal, or salivary glands (Steinfeld et al., 2001; Tsubota et al., 2001; Bedford et al., 2003; Zhu et al., 2016). However, only scarce information is available regarding the role of AQPs in pancreatitis. Ohta et al. (2009) have shown that in the absence of AQP12 the course of cerulein-induced pancreatitis is much worse, probably due to the defect in the secretion of zymogens. In addition, decreased expression of AQP1 and -8 has been found in this pancreatitis model that may also affects the outcome of pancreatitis (Kitami et al., 2007).

The aim of this study is to provide the first detailed characterization regarding the role of AQPs in the pancreas, both under physiological and pathophysiological conditions and to study its relationship to the CFTR $\mathrm{Cl}^{-}$channel. We have chosen to focus on AQP1, since this isoform is abundantly expressed in both acinar and ductal cells of mouse and human pancreas and the role of this AQP has not been evaluated in pancreatitis yet. Using in vitro models, human tissues and transgenic mice, we have shown that AQP1 plays essential role in ductal fluid and $\mathrm{HCO}_{3}{ }^{-}$secretion, lack of CFTR decreases its expression and deletion of AQP1 is strongly associated with increased susceptibility of the gland to pancreatitis.

\section{MATERIALS AND METHODS}

\section{Ethical Approval}

Animal experiments were conducted in accordance with the Guide for the Care and Use of Laboratory Animals (United States, Department of Health and Human Services). In addition, the experimental protocol was approved by the local Ethical Board of the University of Szeged, Hungary and by the National Scientific Ethical Committee on Animal Experimentation (Budapest, Hungary). The use of human tissue was approved by the local ethical committee (University of Szeged, Hungary) and written informed consent was obtained from the patients.

\section{Transgenic Mice}

AQP1 knock out (KO) mice were a kind gift from Dr. Alan Verkman (University of California, CA, United States) and Dr. Alastair Poole (University of Bristol, United Kingdom). CFTR KO mice were kindly supplied by Dr. Ursula Seidler (Hannover Medical School, Hannover, Germany). Animals were kept in standard plastic cages on 12:12 h light-dark cycle at room temperature $\left(23 \pm 1^{\circ} \mathrm{C}\right)$ and had free access to standard or CFTR 
specific laboratory chow and drinking solutions. Functional experiments were performed on litter-matched (age 12-16 weeks, both sexes) wild-type (WT) and AQP1 KO mice. All mice were genotyped prior to the experiments. For genotyping, genomic DNA from the tail was isolated and amplified by traditional PCR.

\section{Human Pancreatic Tissue Samples}

Human pancreatic tissue samples were obtained from autopsy and from surgical resections. Control tissue $(n=5)$ were collected from the tumor-free region of the pancreas of patients with neuroendocrine tumors. Tissue samples from patients with acute necrotizing pancreatitis (ANP; $n=5)$ or CP $(n=5)$ were from autopsy and from surgical resections. The average age of ANP patients was $56 \pm 2.8$ years, and the male/female ratio was 1.5:1. The average age of CP patients was $56.8 \pm 2.8$ years, and the male/female ratio was $4: 1$.

\section{Cell Cultures and Treatments}

Capan-1, Panc-1, and Miapaca-2 cells were obtained from the American Type Culture Collection (Manassas, VA, United States). Capan-1 cells were maintained in Roswell Park Memorial Institute (RPMI)-1640 Medium supplemented with $15 \%(\mathrm{v} / \mathrm{v})$ fetal bovine serum (FBS), 1\% (v/v) L-glutamine and $1 \%(\mathrm{v} / \mathrm{v})$ Penicillin-Streptomycin (PS). Panc-1 and Miapaca2 were maintained in Dulbecco's Modified Eagle's Medium (DMEM) high glucose Medium supplemented with 10\% (v/v) FBS, $1 \%(\mathrm{v} / \mathrm{v})$ L-glutamine, 2.5\% (v/v) horse serum and $1 \%$ (v/v) PS. All three cell lines were kept in a humidified incubator at $37^{\circ} \mathrm{C}$. Cells from passage numbers $20-60$ were used in this study. In case of PCR experiments $10^{6}$, whereas in the case of immunostaining $10^{4}$ cells were seeded into $75 \mathrm{~cm}^{2}$ tissue culture flasks or glass bottom petri dishes, respectively, and incubated for $24 \mathrm{~h}$ at $37^{\circ} \mathrm{C}$. After the incubation, cells were treated with chenodeoxycholic acid (CDCA; 100, 300, and 500 $\mu \mathrm{M}$ ), glycochenodeoxycholic acid (GCDCA; 100, 300, and 500 $\mu \mathrm{M}$ ), ethanol (EtOH; 1, 10, and $100 \mathrm{mM}$ ), palmitoleic acid (POA; 10,100 , and $200 \mu \mathrm{M}$ ) and palmitoleic acid ethyl ester (POAEE; 10,100 and $200 \mu \mathrm{M}$ ) for $6,12,24$, and $48 \mathrm{~h}$ and the mRNA and protein expression were investigated by real-time PCR and immunohistochemistry.

\section{Chemicals and Solutions}

2,7-bis-(2-carboxyethyl)-5(6)-carboxyfluorescein acetoxymethyl ester (BCECF-AM) was from Invitrogen (Eugene, OR, United States). BCECF-AM (2 $\mathrm{mmol} / \mathrm{l})$ were prepared in dimethyl sulfoxide (DMSO) and stored at $-20^{\circ} \mathrm{C}$. POAEE was purchased from Cayman Chemical (Tallinn, Estonia). POA and POAEE were made up as a $10 \mathrm{mM}$ stock solution in DMSO and stored at $-20^{\circ} \mathrm{C}$. Chromatographically pure collagenase was purchased from Worthington (Lakewood, NJ, United States). All other chemicals were obtained from Sigma-Aldrich (Budapest, Hungary).

For microfluorimetry studies, the standard HEPES-buffered solution contained (in $\mathrm{mM}$ ): $130 \mathrm{NaCl}, 5 \mathrm{KCl}, 1 \mathrm{CaCl}_{2}, 1 \mathrm{MgCl}_{2}$, 10 D-glucose and $10 \mathrm{Na}$-HEPES. HEPES-buffered solutions were gassed with $100 \% \mathrm{O}_{2}$ and their $\mathrm{pH}$ was set to 7.4 with $\mathrm{HCl}$. The standard $\mathrm{HCO}_{3}{ }^{-} / \mathrm{CO}_{2}$-buffered solution contained (in $\mathrm{mM}$ ): $115 \mathrm{NaCl}, 25 \mathrm{NaHCO}_{3}, 5 \mathrm{KCl}, 1 \mathrm{CaCl}_{2}, 1 \mathrm{MgCl}_{2}$, and $10 \mathrm{D}$ glucose. The $\mathrm{Cl}^{-}$-free $\mathrm{HCO}_{3}{ }^{-} / \mathrm{CO}_{2}$-buffered solution contained (in $\mathrm{mM}$ ): $115 \mathrm{Na}$-gluconate, $25 \mathrm{NaHCO}_{3}, 6 \mathrm{Ca}$-gluconate, 1 $\mathrm{Mg}$-gluconate, $2.5 \mathrm{~K}_{2} \mathrm{H}$-sulfate, and $10 \mathrm{D}$-glucose. $\mathrm{HCO}_{3}{ }^{-} / \mathrm{CO}_{2}$ buffered solutions were gassed with $95 \% \mathrm{O}_{2} / 5 \% \mathrm{CO}_{2}$ to set the $\mathrm{pH}$ to 7.4 .

\section{Isolation of Pancreatic Ducts and Measurement of Intracellular pH}

Intra/interlobular ducts were isolated from the pancreas of WT and AQP KO mice using the microdissection technique as described previously (Argent et al., 1986). Changes in intracellular $\mathrm{pH}\left(\mathrm{pH}_{\mathrm{i}}\right)$ were detected using the $\mathrm{pH}$-sensitive fluorescence dye, BCECF. Pancreatic ducts were incubated with BCECF-AM $(2 \mu \mathrm{M})$ for 30-60 min, at room temperature. After the incubation, ducts were attached to a cover glass, which formed the base of a perfusion chamber, mounted on the stage of an IX71 live cell imaging fluorescence microscope (Olympus, Budapest, Hungary) and excited at 440 and $490 \mathrm{~nm}$. Emissions were monitored at $530 \mathrm{~nm}$. Five to seven region of interests (ROIs) were examined in each experiment, and one measurement per second was obtained. The 490/440 fluorescence ratio was calibrated to $\mathrm{pH}_{\mathrm{i}}$ using the high $\mathrm{K}^{+}$-nigericin technique, as previously described (Thomas et al., 1979; Hegyi et al., 2004).

\section{Measurement of $\mathrm{HCO}_{3}-$ Secretion}

In order to estimate $\mathrm{HCO}_{3}{ }^{-}$efflux, the activity of the $\mathrm{Cl}^{-} / \mathrm{HCO}_{3}{ }^{-}$exchanger was measured by the $\mathrm{Cl}^{-}$withdrawal technique. Removal of luminal $\mathrm{Cl}^{-}$from the standard $\mathrm{HCO}_{3}-/ \mathrm{CO}_{2}$-buffered solution induced an alkalization in the cells due to the reverse mode of the exchanger. Re-addition of $\mathrm{Cl}^{-}$induces $\mathrm{HCO}_{3}{ }^{-}$secretion via the $\mathrm{Cl}^{-} / \mathrm{HCO}_{3}{ }^{-}$exchanger. Under these conditions the initial rate of acidification reflects the activity of the $\mathrm{Cl}^{-} / \mathrm{HCO}_{3}{ }^{-}$exchanger. In order to evaluate base efflux $\left[-J\left(\mathrm{~B}^{-} / \mathrm{min}\right)\right]$ the following equation was used: $-J\left(\mathrm{~B}^{-} / \mathrm{min}\right)=\Delta \mathrm{pH} / \Delta \mathrm{t} \times \beta_{\text {total }}$, where $\Delta \mathrm{pH} / \Delta \mathrm{t}$ is the rate of acidification measured over the first $60 \mathrm{~s}$ and $\beta_{\text {total }}$ is the total buffering capacity of the cell.

\section{Measurement of in Vitro Ductal Fluid Secretion}

Fluid secretion of intra/interlobular pancreatic ducts was measured using a swelling method, as described previously (Fernandez-Salazar et al., 2004). Briefly, isolated pancreatic ducts were attached to a cover glass which formed the base of a perfusion chamber and mounted on the stage of an IX71 live cell imaging fluorescence microscope. Low magnification, brightfield images were acquired at 1-min intervals using a CCD camera (Hamamatsu ORCA-ER, Olympus, Budapest, Hungary). At the end of each experiment, ducts were perfused with hypotonic solution in order to check the integrity of the duct wall. Ducts that not respond to the hypotonic challenge were excluded from the analysis. Changes in relative luminal volume was analyzed by Scion Image software (Scion Corporation, Frederick, MD, United States) (Fernandez-Salazar et al., 2004; Pascua et al., 2009). 


\section{Measurement of in Vivo Pancreatic Fluid Secretion}

In order to measure pancreatic fluid secretion in vivo, magnetic resonance imaging (MRI) was performed on WT and AQP1 KO mice (Maleth et al., 2015). Animals were allowed free access to pineapple juice $12 \mathrm{~h}$ before the MRI examination. MRI was performed in a 7.1 Tesla animal scanner (Bruker, Ettlingen, Germany). Strong T2-weighted series of the complete abdomen were acquired before and after retroorbital injection of 10 IU units/kg body weight (b.w.) secretin (ChiroStim, ChiRhoClin, Burtonville, MD, United States). The time between injection and MRI was $6 \mathrm{~min}$. The sequences were acquired using the image parameters: TR/TE $4400 / 83 \mathrm{~ms}$; flip angle: $180^{\circ}$; matrix $256 \times 256$; field of view $40 \times 40 \mathrm{~mm}$; bandwidth $315 \mathrm{~Hz} /$ pixel; slice thickness $1 \mathrm{~mm} ; 20$ slices. All image analyses were performed using Osirix (version 5; Pixameo, Bernex, Switzerland). In order to exclude effects of the basal secretion, MRI datasets after and before secretion were subtracted. The created images show the total excretion after secretin stimulation. Excreted fluid is defined as high signal intensity in created images. The fluid excretion into the small intestine was segmented in each slice. The software calculated the volume of the segmented areas. This volume represents the total excreted volume (TEV). In order to minimize artifacts, image noise was reduced.

\section{Measurement of Osmotic Transepithelial Water Permeability}

The osmotic transcellular water movement $\left(\mathrm{P}_{\mathrm{f}}\right)$ was estimated using the cell-impermeant dye, Texas $\operatorname{Red}^{\mathrm{TM}}$ Dextran as previously described (Levin et al., 2006). Briefly, cells $\left(5 \times 10^{5}\right)$ were grown on a polyester permeable support (Transwell, $12 \mathrm{~mm}$ diameter and $0.4 \mu \mathrm{m}$ pore size). Monolayer confluence was checked by measuring the transepithelial electrical resistance $\left(\mathrm{R}_{\mathrm{T}}\right)$ using an EVOM-G Volt/Ohm Meter (World Precision Instruments, Sarasota, FL, United States). Cells were washed with isoosmolar phosphate-buffered saline (PBS) from the basolateral surface and hyperosmolar PBS (complemented with $300 \mathrm{mM}$ D-mannitol and $0.25 \mu \mathrm{g} / \mathrm{mL}$ Texas Red Dextran) from the luminal surface. In some experiments, different concentrations of bile acids were added to the apical solution. Transwells were than placed into a $\mathrm{CO}_{2}$ incubator and $5 \mu \mathrm{l}$ samples were collected from the apical solution at specified time points. Water moves along the osmotic gradient that causes the dilution of the fluorescent dye, in the apical solution. Fluorescence was measured at $595 \mathrm{~nm}$ excitation and $615 \mathrm{~nm}$ emission, using a Fluoro Max-4 spectrofluorometer (Horiba Scientific, Tokyo, Japan). For the calculation of $\mathrm{P}_{\mathrm{f}}$, the following formula was used: $\mathrm{dV}(0) / \mathrm{dt}=\mathrm{P}_{\mathrm{f}}{ }^{*} \mathrm{~S}^{*} \mathrm{v}_{\mathrm{w}}{ }^{*}\left(\Phi_{1}-\Phi_{2}\right)$, where $S$ is the tissue surface area, $V_{W}$ is the partial molar volume of water and $\Phi_{1}-\Phi_{2}$ is the transepithelial osmotic gradient.

\section{Real-Time PCR}

Total RNA was purified from individual cell culture samples using the RNA isolation kit of Macherey-Nagel (Macherey-Nagel,
Düren, Germany). All the preparation steps were carried out according the manufacturer's instructions. RNA samples were stored at $-80^{\circ} \mathrm{C}$ in the presence of $30 \mathrm{U}$ of Prime RNAse inhibitor (Fermentas, Lithuania) for further analysis. The quantity of isolated RNA samples was checked by spectrophotometry (NanoDrop 3.1.0, Rockland, DE, United States). In order to monitor gene expression, QRT-PCR was performed on a RotorGene 3000 instrument (Corbett Research, Sydney, NSW, Australia) using the TaqMan probe sets of specific Aquaporin genes (Applied Biosystems, Foster City, CA, United States). Information about the genes and the TaqMan assays is collected in Table 1. $3 \mu \mathrm{g}$ of total RNA was reverse transcribed using the High-Capacity cDNA Archive Kit (Applied Biosystems, Foster City, CA, United States) according to the manufacturer's instructions in final volume of $30 \mu \mathrm{L}$. The temperature profile of the reverse transcription was the following: $10 \mathrm{~min}$ at room temperature, $2 \mathrm{~h}$ at $37^{\circ} \mathrm{C}, 5 \mathrm{~min}$ on ice and finally $10 \mathrm{~min}$ at $75^{\circ} \mathrm{C}$ for enzyme inactivation. These steps were carried out in a Thermal Cycler machine (MJ Research, Waltham, MA, United States). After dilution with $30 \mu \mathrm{L}$ of water, $1 \mu \mathrm{L}$ of the diluted reaction mix was used as template in the QRT-PCR. For all the reactions TaqMan Universal Master Mix (Applied Biosystems, Foster City, CA, United States) were used according to the manufacturer's instructions. Each reaction mixture (final volume $20 \mu \mathrm{L}$ ) contained $1 \mu \mathrm{L}$ of primer-TaqMan probe mix. The QRT-PCR reactions were carried out under the following conditions: $15 \mathrm{~min}$ at $95^{\circ} \mathrm{C}$ and 45 cycles of $95^{\circ} \mathrm{C}$ for $15 \mathrm{~s}$, $60^{\circ} \mathrm{C}$ for $1 \mathrm{~min}$. Fluorescein dye (FAM) intensity was detected after each cycle. Non-template control sample was used for each PCR run to check the primer-dimer formation. Relative gene expression ratios were calculated as $\Delta \mathrm{Ct}$ values $(\mathrm{Ct}$ values of gene of interest versus $\mathrm{Ct}$ values of human hypoxanthine phosphoribosyltransferase gene). In the case of treatments, relative changes in gene expression were determined using the $\Delta \Delta \mathrm{C}_{\mathrm{T}}$ method as described in Applied Biosystems User Bulletin No. 2 (P/N 4303859). $\Delta \Delta \mathrm{C}_{\mathrm{T}}$ was calculated using the following formula: $\Delta \Delta \mathrm{C}_{\mathrm{T}}=\Delta \mathrm{C}_{\mathrm{T}}$ of treated cells $-\Delta \mathrm{C}_{\mathrm{T}}$ of control, nontreated cells. The $\mathrm{N}$-fold differential expression in the target gene was expressed as $2^{-\Delta \Delta C_{\mathrm{T}}}$. Genes with expression values less than or equal to 0.5 were considered to be down-regulated, whereas values higher than or equal to 2 were considered to be upregulated. Values ranging from 0.51 to 1.99 were not considered to be significant.

\section{Immunocytochemistry}

CAPAN-1 cells were washed with PBS twice and fixed in paraformaldehyde ( $4 \%$ in $\mathrm{PBS}$ ) for $30 \mathrm{~min}$ at room temperature (RT). In order to avoid non-specific antibody binding cells were incubated with $10 \%$ donkey serum and $1 \%$ BSA for further $30 \mathrm{~min}$. After blocking, cells were incubated with primary AQPs human, polyclonal antibodies (1:100 dilutions; Abcam, Cambridge, United Kingdom) at $4^{\circ} \mathrm{C}$ overnight. Petri dishes were then washed with PBS and incubated with FITC-conjugated AffiniPure Donkey AntiRabbit IgG secondary antibody (1:400 dilutions; DAKO, Milan, Italy) for $60 \mathrm{~min}$ at RT. Nuclei were counterstained with Dapi. Dishes were then mounted and observed by a 
TABLE 1 | TaqMan assays used for the investigation of AQP expression.

\begin{tabular}{|c|c|c|c|}
\hline Definition & Gene symbol & Ref Seq Acc. number & ABI TaqMan assay ID \\
\hline Homo sapiens aquaporin 1 (Colton blood group) & AQP1 & NM_198098 & Hs00166067_m1 \\
\hline Homo sapiens aquaporin 2 (collecting duct) & AQP2 & NM_000486 & Hs00166640_m1 \\
\hline Homo sapiens aquaporin 3 (Gill blood group) & AQP3 & NM_004925 & Hs00185020_m1 \\
\hline Homo sapiens aquaporin 4 & AQP4 & NM_001650 & Hs00242341_m1 \\
\hline Homo sapiens aquaporin 5 & AQP5 & NM_001651 & Hs00387048_m1 \\
\hline Homo sapiens aquaporin 6, kidney specific & AQP6 & NM_001652 & Hs01546883_m1 \\
\hline Homo sapiens aquaporin 7 & AQP7 & NM_001170 & Hs00357359_m1 \\
\hline Homo sapiens aquaporin 8 & AQP8 & NM_001169 & Hs00154124_m1 \\
\hline Homo sapiens aquaporin 9 & AQP9 & NM_020980 & Hs00175573_m1 \\
\hline Homo sapiens aquaporin 10 & AQP10 & NM_080429 & Hs00369738_m1 \\
\hline Homo sapiens aquaporin 11 & AQP11 & NM_173039 & Hs00542681_m1 \\
\hline Homo sapiens aquaporin $12 \mathrm{~A}$ & AQP12A & NM_198998 & Hs01651303_m1 \\
\hline Homo sapiens aquaporin 12B & AQP12B & NM_001102467.1 & \\
\hline
\end{tabular}

Fluowiew 10i-W confocal microscopy (Olympus, Budapest, Hungary).

\section{Immunohistochemistry}

Paraffin-embedded, 3- to 4- $\mu$ m-thick sections of surgically removed resection specimens and autopsy tissue samples were used for immunohistochemistry. After deparaffinization of tissue samples with EZ Prep Concentrate 10X (Ventana Medical Systems, Tucson, AZ, United States), endogenous peroxidase blocking and antigen retrieval (CC1; Ventana Medical Systems), pancreas sections were incubated with polyclonal AQP1 antibody (1:100 dilution; Alomone Labs, Jerusalem, Israel) overnight at $4^{\circ} \mathrm{C}$. Immunohistochemical staining was performed with horseradish peroxidase multimer-based, biotin-free detection technique according to the protocol of the automated Ventana system (Ventana Benchmark XT; Ventana Medical Systems). For visualization, the UltraView Universal diaminobenzidine (DAB) Detection Kit (Ventana Medical Systems) was applied. Sections from human pancreas were used as positive controls. For negative control, primary antibodies were substituted with antibody diluent (Ventana Medical Systems). The stained slides were digitized with Mirax Pannoramic MIDI and Mirax Pannoramic SCAN digital slide scanners (3DHistech Ltd., Budapest, Hungary).

Cryosections from WT, AQP1, and CFTR KO mice pancreas were fixed in 2\% paraformaldehyde, permeabilized in $10 \%$ Tween 20 -sodium citrate, blocked with $5 \%$ goat serum followed by immunofluorescent double staining for AQP1 mouse monoclonal antibody (1:500 dilutions; Thermo Fisher, Rockford, IL, United States) and CFTR rabbit polyclonal antibody (1:100 dilutions; Alomone Labs, Jerusalem, Israel) at $4^{\circ} \mathrm{C}$, overnight. Following washing, sections were incubated with secondary antibodies goat-anti-mouse (Alexa fluor 488, Thermo Fisher, Rockford, IL, United States) and goat-anti-rabbit (Alexa fluor 568, Thermo Fisher, Rockford, IL, United States) for $2 \mathrm{~h}$ at room temperature in the dark. Nuclei were counterstained with Dapi. Sections were then mounted and analyzed using a Zeiss LSM 880 confocal laser scanning microscope (Carl Zeiss Technika Kft., Budaörs, Hungary).

\section{Quantification of the Immunostainings}

In order to quantify AQP1 or CFTR positively stained area, 10-12 representative, digital images were taken from the human (normal pancreas, AP and CP) and mice (WT, AQP1 KO and CFTR KO) pancreas sections and from Capan-1 cells. Pictures were than converted to gray scale (16-bit) and thresholded in order to select the positively stained area, using the ImageJ software. The intensity of DAB (human pancreas) or fluorescence signal (mice pancreas and Capan-1 cells) varied on an arbitrary scale from 0 to 255 , where 0 is the negative staining (white pixels) and 255 is the maximal staining (black pixels). In the human samples, the mean integrated density of the positively stained area was normalized to the mean integrated density of the total image and converted into percentage. In the cell line, the mean integrated density of the positively stained area was normalized to the total cell number and expressed in arbitrary units. In the mice samples, the ductal area was selected and the total fluorescence intensity was calculated and summarized which was than normalized to the ductal area $\left(\mu \mathrm{m}^{2}\right)$ and expressed in arbitrary units $/ \mu \mathrm{m}^{2}$. Comparison of each groups were calculated by one-way ANOVA, followed by the Holm-Sidak method. Statistical significance was defined as $p \leq 0.05$. Data are expressed as means \pm SEM.

\section{Induction of Acute Pancreatitis in Mice}

Acute pancreatitis (AP) was induced in mice by hourly (10 times) intraperitoneal (i.p.) injections of cerulein $(50 \mu \mathrm{g} / \mathrm{kg})$ (Niederau et al., 1985; Ding et al., 2003; Pallagi et al., 2014) following anesthesia with i.p. $85 \mathrm{mg} / \mathrm{bwkg}$ pentobarbital (Bimeda MTC, Cambridge, ON, Canada). The control animals received the same amount of saline. Two hours after the final injection, mice were euthanized by pentobarbital overdose $(200 \mathrm{mg} / \mathrm{bwkg}$ i.p.). Animals were exsanguinated through the cardiac puncture and the pancreata were immediately removed. The collected blood was centrifuged at $4^{\circ} \mathrm{C}$ with $2500 \mathrm{RCF}$ for $15 \mathrm{~min}$ and the sera were stored at $-20^{\circ} \mathrm{C}$ until use. The pancreas was trimmed from fat and lymphatic tissue, put into $6 \%$ neutral formaldehyde solution and stored at $-80^{\circ} \mathrm{C}$ until use. Serum amylase activity was measured with a commercial colorimetric kit 
(Diagnosticum, Budapest, Hungary) with a FLUOstar OPTIMA (BMG Labtech, Budapest, Hungary) microplate reader at $405 \mathrm{~nm}$. The formaldehyde-fixed pancreatic tissue was embedded in paraffin blocks, cut into $3 \mu \mathrm{m}$ thick sections and stained for hematoxylin-eosin using standard techniques and viewed by light microscopy. A semiquantitative scoring system was used to evaluate the presence of edema, the rate of necrosis and infiltration of inflammatory cells according to the following scoring system (Kui et al., 2015): Edema (0: none, 1: patchy interlobular; 2: diffuse interlobular; 3: diffuse interlobular, and intraacinar), necrosis (0: none, 1: patchy interlobular; 2 : diffuse interlobular; 3: diffuse interlobular and patchy intraacinar; 4: diffuse interlobular and intraacinar) and leukocytic infiltration (0: none; 1: patchy interlobular; 2 : diffuse interlobular; 3 : diffuse interlobular and intraacinar). The rate of necrosis was expressed as percentage of the total analyzed pancreatic area.

\section{Statistical Analysis}

Data are expressed as means \pm SEM. In the case of pancreatic fluid and $\mathrm{HCO}_{3}{ }^{-}$secretion measurements, significant difference between groups was determined by ANOVA. Statistical analysis of the immunohistochemical data was performed using the Student's $t$-test. Probability values of $p \leq 0.05$ were accepted as being significant.

\section{RESULTS}

\section{Capan-1 Cells Express Functionally Active AQPs}

The relative gene expressions of AQP isoforms were studied in different pancreatic ductal cell lines (Capan-1, Panc-1, and Miapaca-2) using RT-PCR and TaqMan primer-probe sets, specific for AQP1-AQP12 isoforms (Table 1). Expressions of AQPs were investigated at different time points $(6,12,24$, and $48 \mathrm{~h}) 24 \mathrm{~h}$ after plating the cells, and as an internal gene, human hypoxanthine phosphoribosyltransferase (HPRT) was used. RTPCR analysis revealed that among the three cell lines, AQPs were expressed at the highest level in Capan-1 cells, whereas in Panc-1 and Miapaca-2 the expression of AQPs was hardly detectable (Figures 1A-C). In Capan-1 cells high levels of AQP1, -3 , and -5 was detected, which is in agreement with previous observations (Burghardt et al., 2003). The presence of these isoforms was also confirmed at protein level in the Capan-1 cells (Figure 1D). In order to test that the AQPs expressed on Capan1 cells are functionally active, we investigated the transepithelial water flow in the presence and absence of the non-specific AQP inhibitor $\mathrm{HgCl}_{2}$. As shown in Figures 1E,F, administration of $0.3 \mathrm{mM} \mathrm{HgCl}_{2}$ to the apical solution inhibited the osmotic water movement by $52.6 \pm 2.4 \%$, indicating that significant proportion of water is transported through AQPs.

\section{AQP1 Is Essential for Pancreatic Ductal Fluid and $\mathrm{HCO}_{3}-$ Secretion}

Aquaporins are involved in transepithelial water transport in numerous tissues (Tradtrantip et al., 2009). In the next step, we investigated the role of this protein in pancreatic ductal fluid secretion using gene modified mice. Using traditional PCR we have shown the presence of AQP1 both in the isolated, intra/interlobular pancreatic ducts and the total pancreas (Figure 2A). Slight expression of AQP5 was also detected in the pancreatic ducts, whereas the mRNA presence of AQP8 and -12 was only observed in the total pancreas (data not shown). We decided to characterize the role of AQP1, since this isoform is constitutively expressed in both the ductal and acinar cells and also can be found in the mouse and human pancreas (Table 2). In contrast to AQP1, AQP3 is not expressed in the mouse pancreas and this isoform is either involved in tumor progression than pancreatitis (Direito et al., 2017; Huang et al., 2017). Although AQP5 is present in the intra/interlobular pancreatic ducts of mice and human, and probably plays role in ductal fluid secretion (Burghardt et al., 2003), KO animal for this isoform was not available to us. Table 2 summarizes the presence of AQP isoforms in mice and human.

The rate of fluid secretion was measured over 30-40 min using sealed ducts and the swelling technique. Initially, ducts were perfused with the standard HEPES-buffered solution and then perfusion was switched to $\mathrm{HCO}_{3}-/ \mathrm{CO}_{2}$-buffered solution to initiate $\mathrm{HCO}_{3}{ }^{-}$-dependent secretion. As shown in Figure 2B, the presence of $\mathrm{HCO}_{3}{ }^{-}$induced a dynamic swelling of the WT ducts as a result of fluid secretion into the closed luminal space. In contrast, ducts from AQP1 KO mice showed no or only a slight response to $\mathrm{HCO}_{3}{ }^{-}$. Under stimulated conditions (forskolin; $10 \mu \mathrm{M}$ ), the rate of fluid secretion further increased in the WT ducts, whereas in the case of KO ducts, no response was detected. Figure 2C summarizes the relative luminal volume changes in WT and $\mathrm{KO}$ ducts after forskolin stimulation. As shown in Figure 2C, the relative luminal volume increase reduced by $89.9 \pm 6.2 \%$ in the absence of AQP1, indicating that this AQP isoform plays an essential role in fluid secretion.

We also investigated the rate of pancreatic fluid secretion in vivo by MRI cholangiopancreatography in anesthetized mice. Fluid secretion was stimulated by retroorbital administration of $10 \mathrm{U} / \mathrm{kg}$ b.w. secretin, and then TEV was measured in WT and $\mathrm{KO}$ animals. Similarly, to the in vitro measurements, the pancreatic fluid secretion significantly reduced in AQP1 KO $\left(0.0041 \mathrm{TEV} / \mathrm{cm}^{3}\right)$ vs. WT $\left(0.023 \mathrm{TEV} / \mathrm{cm}^{3}\right)$ mice (Figures 2D,E).

Next, we were curious whether the decreased ductal fluid secretion is also associated with impaired $\mathrm{HCO}_{3}{ }^{-}$efflux in AQP1 $\mathrm{KO}$ animals. For the measurement of ductal $\mathrm{HCO}_{3}{ }^{-}$secretion, intra/interlobular pancreatic ducts were isolated from the pancreas of WT and AQP1 KO mice. Ducts were perfused both from the luminal and basolateral membrane with $\mathrm{HCO}_{3}{ }^{-} / \mathrm{CO}_{2}$ buffered solution. Removal of $\mathrm{Cl}^{-}$from the apical solution induced an alkalisation inside the cells, due to the reverse mode of the $\mathrm{Cl}^{-} / \mathrm{HCO}_{3}{ }^{-}$exchanger. Addition of $\mathrm{Cl}^{-}$back to the external solution decreased the $\mathrm{pH}_{\mathrm{i}}$ indicating $\mathrm{HCO}_{3}{ }^{-}$efflux through the exchanger. Under these conditions, the initial rate of recovery from alkalosis reflects the activity of the $\mathrm{Cl}^{-} / \mathrm{HCO}_{3}{ }^{-}$exchanger $\left[\left(-J\left(\mathrm{~B}^{-} / \mathrm{min}\right)\right]\right.$. Using this technique, we showed that the rate of acidification significantly reduced in AQP1 KO (42 $\pm 3.2 \%)$ vs. WT ducts indicating that pancreatic ductal $\mathrm{HCO}_{3}{ }^{-}$secretion is impaired in the absence of AQP1 (Figures 2F,G). 

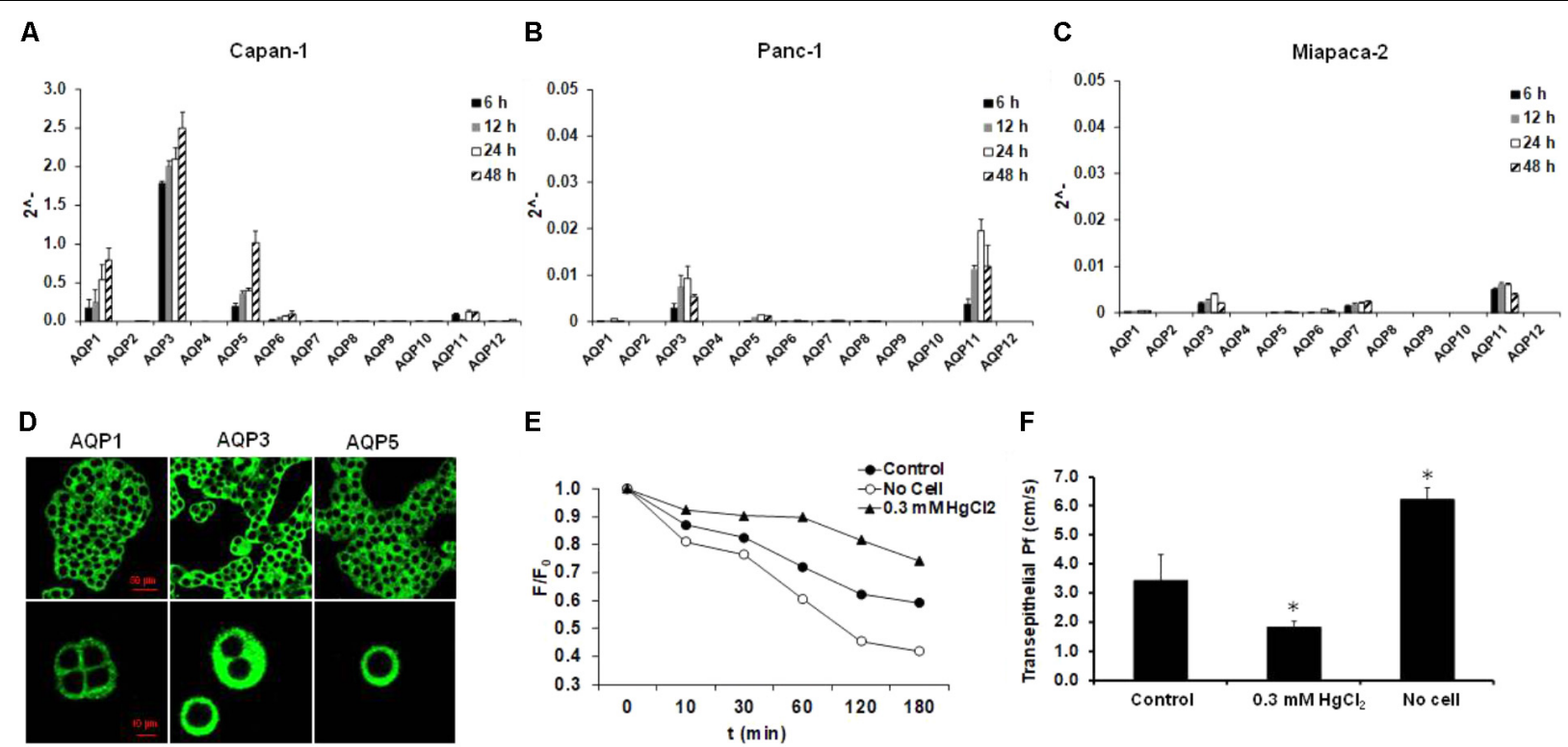

E

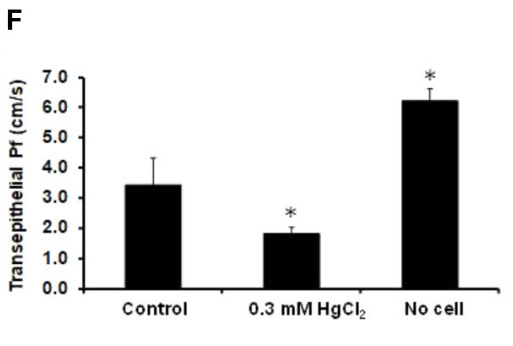

FIGURE 1 | Expression and activity of AQPs in pancreatic ductal cells. Expression of different AQP isoforms was investigated by real-time PCR in (A) Capan-1, (B) Panc-1, and (C) Miapaca-2 pancreatic ductal cell lines, 6, 12, 24, and $48 \mathrm{~h}$ after the plating the cells. Data represent mean \pm SEM of three, independent experiments. (D) Immunofluorescence staining of Capan-1 cells using FITC-conjugated anti-AQP1, -3, and -5 antibodies. (E) Osmotic water movement was investigated in Capan-1 cells. Representative graph shows changes in the fluorescence intensity of the apical solution, at different time points, in the present (triangle) and absence (black circle) of luminal $\mathrm{HgCl}_{2}(0.3 \mathrm{mM})$. To estimate the changes, the fluorescence intensity $\mathbf{( F )}$ was normalized to the initial value $\left(\mathrm{F}_{0}\right)$. Transwell without cells was used for absolute positive control (open circle). (F) Summary of the $\mathrm{P}_{\mathrm{f}}$ values obtained from the experiments in (E). Data represent mean \pm SEM of three, independent experiments. ${ }^{*} p \leq 0.05$ vs. Control.

\section{AQP1 Expression Decreased in CFTR KO Mice}

The decreased $\mathrm{HCO}_{3}{ }^{-}$secretion in AQP1 $\mathrm{KO}$ mice indicates that beside the transport of water, AQP1 interacts with one or more ion transporters which are involved in $\mathrm{HCO}_{3}{ }^{-}$secretion. The CFTR $\mathrm{Cl}^{-}$channel plays essential role in ductal $\mathrm{HCO}_{3}{ }^{-}$ secretion by maintaining a luminal $\left[\mathrm{Cl}^{-}\right]$which is necessary for $\mathrm{HCO}_{3}{ }^{-}$efflux through the $\mathrm{Cl}^{-} / \mathrm{HCO}_{3}{ }^{-}$exchanger. Several studies presume a physical interaction between the CFTR $\mathrm{Cl}^{-}$ channel and certain AQP isoforms (Schreiber et al., 1999; Cheung et al., 2003; Jesus et al., 2014a,b). Colocalization of this two channel has also been found in the human pancreas (Burghardt et al., 2003). In the following step, we performed immunostaining on the pancreas of AQP1 and CFTR KO mice in order to characterize the possible relation between the two channels. In WT mice, AQP1 expression was detected throughout the whole plasma membrane, whereas expression of CFTR exclusively localized to the apical membrane of the ducts (Figure 3A). The absence of AQP1 caused a slight but not significant decrease in the expression of CFTR, indicating that the impaired $\mathrm{HCO}_{3}{ }^{-}$ secretion in the AQP1 KO mice is not due to the decreased expression of CFTR (Figure 3A, middle line). Interestingly we have found that expression of AQP1 dramatically decreased in the intra/interlobular ducts of CFTR KO mice, especially at the apical membrane (Figure 3A, bottom line and Figure $3 \mathbf{B}$ ). We have also found that the absence of CFTR did not affect the expression of AQP1 in the blood vessels indicating that some kind of interaction may exist between these two channels in the pancreatic ductal cells. Although further investigations are needed to clarify whether the two channels are able to regulate each others function, expression or trafficking.

\section{Expression and Function of AQPs Significantly Decreased After Bile Acid Treatment}

In the next step, we studied the effect of pancreatitis-inducing factors on the expression of AQP1. Gallstone obstruction and heavy alcohol consumption are the two major causes of pancreatitis. Capan-1 cells were treated with CDCA and GCDCA (100, 300, and $500 \mu \mathrm{M})$ (Venglovecz et al., 2008, 2011; Muili et al., 2013), EtOH (1, 10, and $100 \mathrm{mM})$, POA and POAEE $(10,100$, and $200 \mu \mathrm{M})$ (Criddle et al., 2006; Judak et al., 2014; Maleth et al., 2015) for 6, 12, 24, and $48 \mathrm{~h}$ and the mRNA expressions of AQP1 were analyzed by RT-PCR. Among the investigated agents, CDCA had the most marked effect, it dose- and time-dependently decreased the expression of AQP1 (Figure 4A). GCDCA, POA, and POAEE caused a significant decrease at 12 and $24 \mathrm{~h}$, primarily at higher doses, which partially regenerated after $48 \mathrm{~h}$ in the continuous presence of the agents (Supplementary Figures 1A-C). In contrast to bile acids, EtOH initially $(24 \mathrm{~h})$ increased the expression of AQP1 that was followed by a decrease (Supplementary Figure 1D). In order to decide whether the downregulating effect of CDCA can also be observed at protein level, we performed immunostaining on the CDCA-treated cells, using specific antibodies against AQP1. CDCA dose- and time-dependently 
A

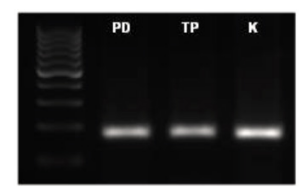

B

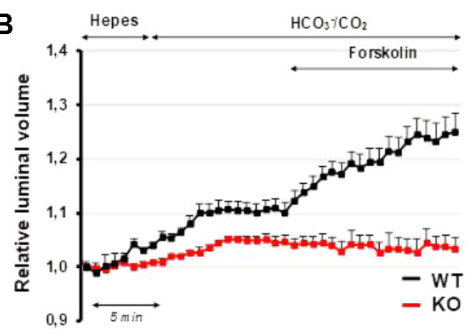

C

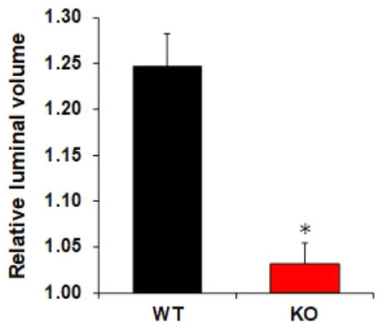

D

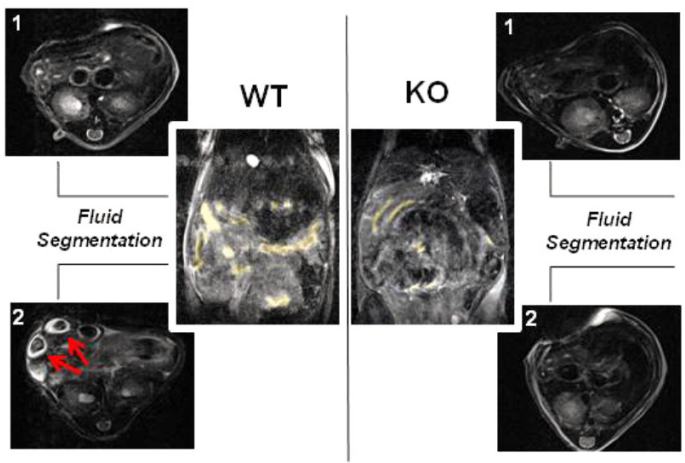

$\mathbf{F}$

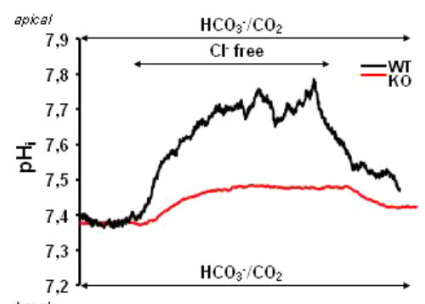

E

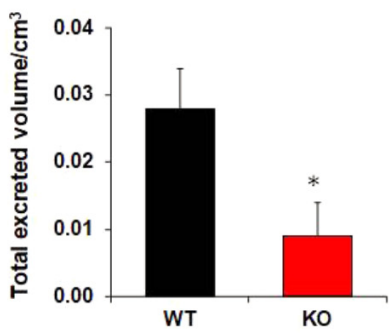

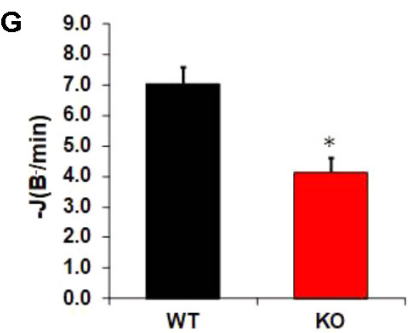

FIGURE 2 | Pancreatic ductal $\mathrm{HCO}_{3}{ }^{-}$and fluid secretion in AQP1 knock out mice. (A) Traditional PCR shows the presence of AQP1 mRNA both in the isolated pancreatic ducts (PD) and in the total pancreas (TP). As positive control, kidney was used (K). (B) Pancreatic ductal fluid secretion was measured on isolated pancreatic ducts from wild type (WT, black line) and AQP1 knock out (KO, red line) mice. Changes in the relative luminal volume of the ducts was measured by videomicroscopy and analyzed by Scion Image software. (C) Changes in the relative luminal volume of the pancreatic ducts of AQP1 WT and KO mice measured at the final time point (40 min). Data are shown as means \pm SEM. $n=4-8$ ducts/groups. ${ }^{*} p \leq 0.05$ vs. WT. (D) Reconstructed images of the duodenal filling before (1) and after (2) secretin stimulation. Yellow color shows the presence of fluid. Red arrows indicate excreted volume in the small bowel after stimulation with secretin. In the case of KO mice, the secretin-stimulated fluid secretion significantly decreased. (E) In vivo pancreatic fluid secretion was measured as total excreted volume (TEV) using small animal magnetic resonance imaging. Raw data were acquired in axial and coronar view. Data are shown as means \pm SEM. $n=4$ animals/groups. ${ }^{*} p \leq 0.05$ vs. WT. (F) Representative experimental traces show pancreatic ductal $\mathrm{HCO}_{3}{ }^{-}$secretion in AQP1 WT (black line) and AQP1 KO (red line) mice. (G) Base flux $\left[-J\left(\mathrm{~B}^{-} / \mathrm{min}\right)\right]$ was calculated from the $\Delta \mathrm{pH} / \Delta \mathrm{t}$ obtained by linear regression analysis of $\mathrm{pH}_{\mathrm{i}}$ measurements made over the first $60 \mathrm{~s}$ after readdition of extracellular $\mathrm{Cl}^{-}$. Means \pm SEM are from 20 ROls of four ducts. ${ }^{*} p \leq 0.05 \mathrm{vs}$. WT.

TABLE 2 | mRNA expression of AQP isoforms in mice and human.

\begin{tabular}{|c|c|c|c|c|c|}
\hline Known isoforms & $\begin{array}{l}\text { Isoforms in mice } \\
\text { pancreas }\end{array}$ & $\begin{array}{l}\text { Isoforms in mice } \\
\text { pancreatic ducts }\end{array}$ & $\begin{array}{l}\text { Isoforms in human } \\
\text { pancreas }\end{array}$ & $\begin{array}{l}\text { Isoforms in human } \\
\text { pancreatic ducts }\end{array}$ & Available KO mice \\
\hline AQPO & - & - & - & - & - \\
\hline AQP1 & $\sqrt{ }$ & $\sqrt{ }$ & $\sqrt{ }$ & $\sqrt{ }$ & $\sqrt{ }$ \\
\hline AQP2 & - & - & - & - & - \\
\hline AQP3 & - & - & - & $\sqrt{ }$ & - \\
\hline AQP4 & - & - & - & - & $\sqrt{ }$ \\
\hline AQP5 & $\sqrt{ }$ & $\sqrt{ }$ & $\sqrt{ }$ & $\sqrt{ }$ & - \\
\hline AQP6 & - & - & - & - & - \\
\hline AQP7 & - & - & - & - & - \\
\hline AQP8 & $\sqrt{ }$ & - & $\sqrt{ }$ & - & - \\
\hline AQP9 & - & - & - & - & - \\
\hline AQP10 & - & - & - & - & - \\
\hline AQP11 & - & - & - & - & - \\
\hline AQP12 & $\sqrt{ }$ & - & $\sqrt{ }$ & - & - \\
\hline
\end{tabular}

decreased the protein expression of AQP1 was consistent with the PCR data (Figure 4B). Representative ICC pictures show that incubation with $500 \mu \mathrm{M}$ CDCA time-dependently decreased the AQP expression in the cells (Figure 4C). The effect of
CDCA on the activity of AQPs was also investigated. As shown in Figure 4D, 100 and $300 \mu \mathrm{M}$ CDCA had no effect on the transepithelial water movement, however, at $500 \mu \mathrm{M} \mathrm{P}_{\mathrm{f}}$ was significantly impaired. 

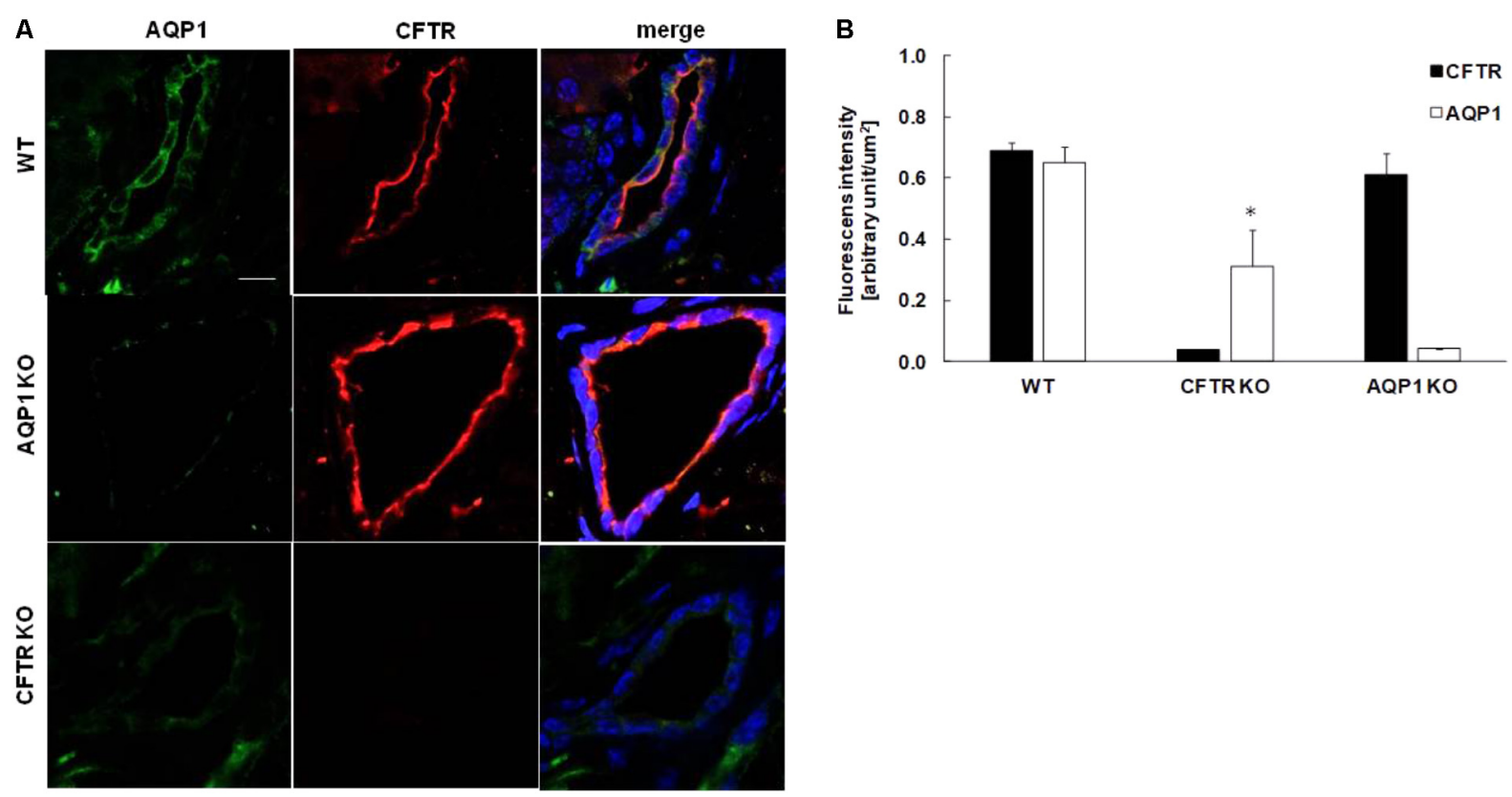

FIGURE 3 | Expression of AQP1 and CFTR in the pancreas of knock out mice. (A) Representative immunofluorescence staining of AQP1 and CFTR in wild type (WT, upper line), AQP1 knock out (AQP1 KO, middle line) and CFTR KO (bottom line) mice. Pictures were taken at $40 \times$ magnification. Pancreas slices were excited at 405 (Dapi), 488 (Alexa fluor 488) and 568 (Alexa fluor 568) nm and emissions were collected at 453, 516, and $603 \mathrm{~nm}$, respectively. Scale bar represents $10 \mu \mathrm{m}$. (B) Summary bar chart shows the mean fluorescence intensity in the ductal cells normalized to the ductal area and expressed in arbitrary units/ $\mu \mathrm{m}^{2}$. Data are presented as means \pm SEM. * $p \leq 0.05$ vs. WT (AQP1), $n=5$.

\section{Acute Pancreatitis Is Aggravated in AQP1 KO Mice}

In order to test the hypothesis that AQP1 may be involved in the pathomechanism of pancreatitis, we investigated the severity of cerulein-induced AP in WT and AQP1 KO mice. WT and AQP1 KO mice were given 10 hourly injections of either physiological saline (control) or supramaximal doses of cerulein (50 $\mu \mathrm{g} / \mathrm{kg}$ per injection) i.p. to induce AP. After saline injection, the pancreas had normal histology in both WT and $\mathrm{KO}$ animals. In contrast, i.p. injections of cerulein caused extensive cell damage both in the WT and KO animals. The extension of pancreatic necrosis were markedly higher in the AQP1 KO $(25 \pm 2.8 \%)$ vs. WT mice (12.1 $\pm 3.2 \%)$, whereas no differences were observed in the extent of edema and in the infiltration of inflammatory cells (Figures 5A-C). Serum amylase activities were significantly higher in $\mathrm{KO}(1605 \pm 6$ $\mathrm{U} / \mathrm{l})$ vs. WT mice $(1285 \pm 51 \mathrm{U} / \mathrm{l})$ after the induction of AP (Figure 5D). As shown on the representative histological images (Figure 5E), the rate of pancreatic necrosis was more extensive in AQP1 KO mice. Overall, these results indicate that in the absence of AQP1 the course of pancreatitis is more severe.

\section{Expression of AQP1 Is Decreased in Acute and Chronic Pancreatitis}

Since we found that the lack of AQP1 exacerbates the course of cerulein-induced pancreatitis in mice, we tested whether this water channel is also involved in the pathomechanism of pancreatitis in humans. Therefore, in the next step we investigated the expression of AQP1 in pancreatic tissues samples obtained from five patients with ANP and five patients with CP. Control pancreatic tissue were obtained from tumorfree tissue surrounding neuroendocrine pancreatic tumors. In order to localize AQP1, IHC was performed. In the normal pancreas, strong AQP1 immunoreactivity was detected in the intra/interlobular and intercalated ducts, the acinar and centroacinar cells (Figures 6A-C). The staining in acinar cells and smaller ducts is mainly localized to the lateral and apical surface of the cells (Figures 6B,C). In the interlobular ducts, the apical plasma membrane was positive to AQP1 with some cytoplasmic staining, whereas Langerhans islets were completely negative for AQP1. These results are consistent with previous observations (Burghardt et al., 2003). In the ANP and CP pancreatic tissue sections the expression of AQP1 strongly reduced in the interlobular ducts, whereas intralobular and intercalated ducts still exhibited weak to moderate AQP1 immunoreactivity (Figures 6D-G). In case of acinar cells, the expression of AQP1 slightly decreased in the inflamed pancreas, especially in ANP. Quantification of DAB intensity showed that AQP1 staining was significantly higher in normal pancreas vs. the ANP or CP groups (Figure $6 \mathbf{H}$ ).

\section{DISCUSSION}

Acute and chronic pancreatitis are serious disorders characterized by inflammation and injury of the gland. 

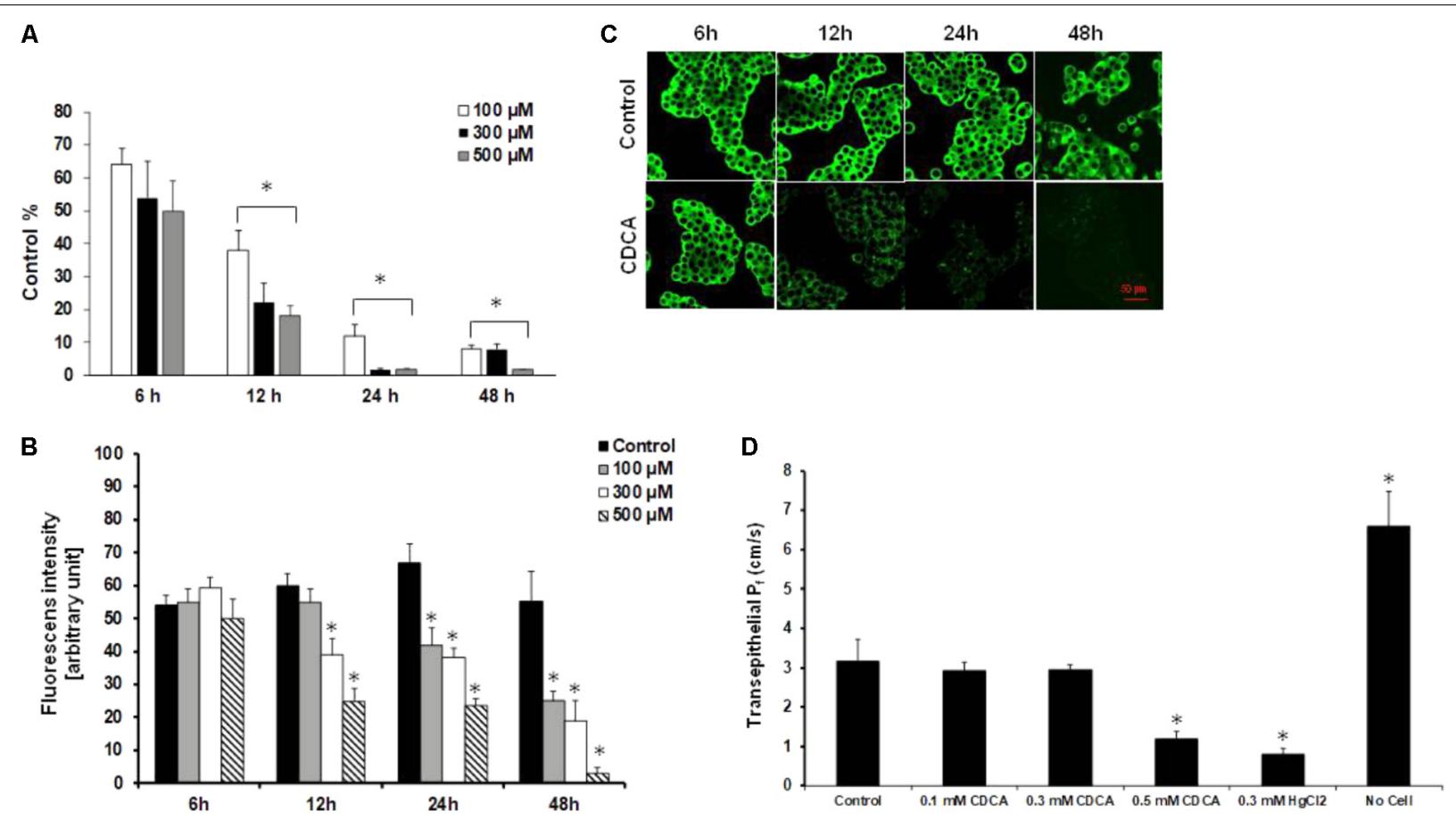

FIGURE 4 | Effect of chenodeoxycholic acid on the expression and activity of AQP1. (A) Capan-1 cells were treated with chenodeoxycholic acid (CDCA) for 6, 12, 24, and $48 \mathrm{~h}$ and the relative gene expression of AQP1 was investigated by real-time PCR. Data represent mean \pm SEM of three, independent experiments. (B) Protein expression of AQP1 was investigated by immunocytochemistry in Capan-1 cells. Protein expression was measured from the mean fluorescence intensity, normalized to the total cell number and expressed in arbitrary units. Data represent mean \pm SEM of three, independent experiments. (C) Representative immunofluorescence staining of Capan-1 cells show the expression of AQP1 after the treatment with CDCA (500 $\mu$ M) for 6, 12, 24, and 48 h. (D) Summary bar chart shows the changes in osmotic transcellular water movement $\left(\mathrm{P}_{\mathrm{f}}\right)$ of Capan-1 cells in the presence of various concentrations of CDCA. Transwell without cells was used for absolute positive control. Data represent mean \pm SEM of three, independent experiments. ${ }^{*} p \leq 0.05$ vs. Control.

Gallstones and heavy alcohol consumption are responsible for approximately $80 \%$ of all AP patients (Parniczky et al., 2016), whereas alcohol abuse is the primary cause of CP (Szucs et al., 2017). Although the therapy improved a lot in the last few years the morbidity and mortality of pancreatitis is still excessively high (Parniczky et al., 2016). Defects in ductal fluid secretion have been proposed as an important factor in the pathomechanism of pancreatitis (Hegyi et al., 2011b). This fluid is high in $\mathrm{HCO}_{3}{ }^{-}$ which prevents the premature activation of zymogens and provides an optimal environment in the duodenum for the action of digestive enzymes. The secreted fluid is also beneficial from the point of view that washing out the potential toxic factors such as activated digestive enzymes or bile acids from the ductal tree and therefore prevents the pancreas from their damaging effects (Venglovecz et al., 2008). AQP1 is a water channel that extensively expressed in the acinar and ductal cells where mediates pancreatic fluid production. Several studies indicate that altered expression or localization of AQPs associates with different gastrointestinal disorders, such as gastritis or diarrhea; therefore, many studies have been conducted to identify the specific role of particular AQP isoforms. Although, AQP1 plays an essential role in pancreatic physiology its function under pathological conditions is not known.

In the present study, we showed that (i) bile acids strongly decrease the expression of AQPs in pancreatic ductal cells, (ii) chronic or acute inflammation of the pancreas is associated with decreased expression of AQP1, (iii) the absence of AQP1 reduces ductal fluid and $\mathrm{HCO}_{3}{ }^{-}$secretion, (iv) and induces a more severe pancreatitis in a cerulein-induced pancreatitis model.

Basically there are two ways for water transport. One is the paracellular way along the osmotic gradient generated by the ion transporters and the other one is the transcellular pathway through the AQPs. In case of the pancreatic ducts, it is not clear which pathway is the dominant. The pancreatic ductal epithelium is a moderately leaky epithelium which favors the paracellular movement of water (Novak and Greger, 1988). Nevertheless, administration of luminal $\mathrm{Hg}^{2+}$, a non-specific inhibitor of AQPs, decreased ductal fluid secretion by more than $90 \%$ in rat pancreatic ducts, which strengthen the importance of transcellular pathway (Ko et al., 2002). In the present study, we demonstrated that pancreatic ductal cells express functionally active AQPs. We have identified the presence of AQP1, -3, and -5 on the ductal cells that are consistent with previous observations (Burghardt et al., 2003). Treatment of the cells with pancreatitis-inducing agents, mostly decreased the expression of AQPs both at mRNA and protein levels. Among the investigated agents, CDCA had the biggest effect, which dose- and timedependently decreased the expression of AQP1. These data are partly consistent with previous findings in the colon, where bile acid treatment reduced the protein expression of AQP3, 

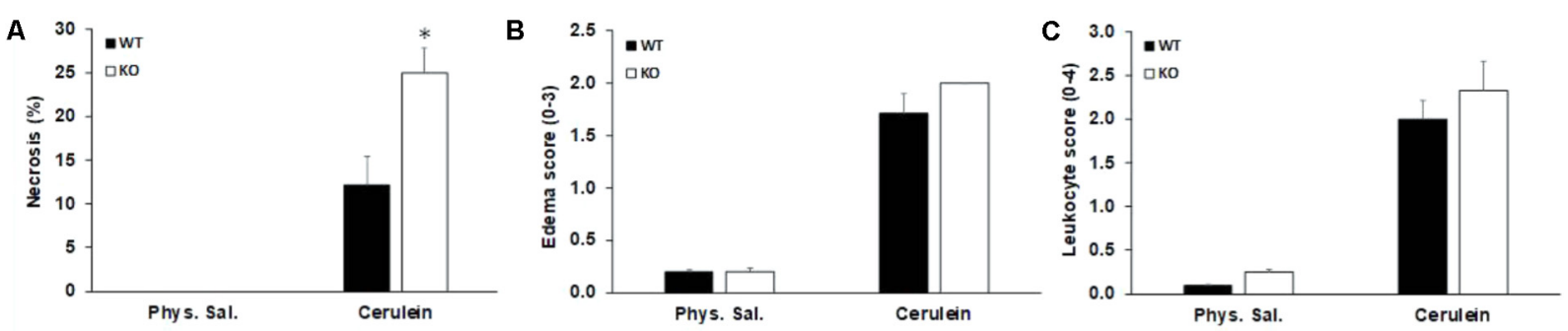

D

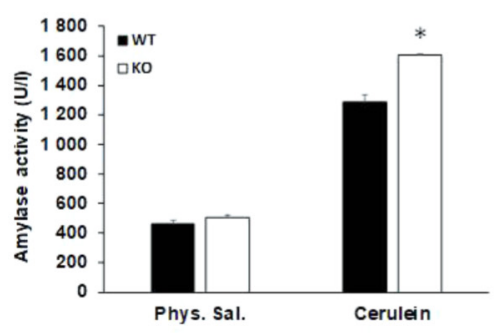

E

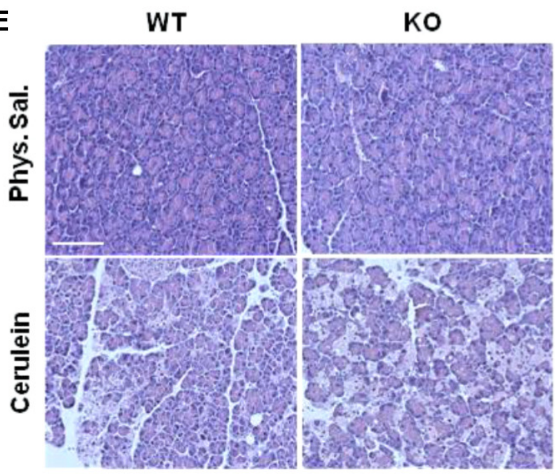

FIGURE 5 | Characterization of acute pancreatitis in AQP1 knock out mice. (A) Pancreatic cell necrosis, (B) edema, (C) leukocyte infiltration, and (D) serum amylase activity (U/l) were measured in wild-type (WT) and AQP1 knock out (KO) mice after induction of pancreatitis. Control animals received the same amount of saline. Data are presented as means \pm SEM. ${ }^{*} p \leq 0.05$ vs. WT. (E) Representative histological images show pancreatic sections from control (physiological saline) and cerulein-treated animals. Phys sal., physiological saline. Scale bar represents $50 \mu \mathrm{m}$.

whereas increased the levels of AQP7 and -8 (Yde et al., 2016). In case of $\mathrm{EtOH}$, the expression of AQPs initially increased (presumably due to a compensatory mechanism), then a decrease was observed, similarly to the rat stomach, where intragastrical administration of $1 \mathrm{mM}$ EtOH caused analogous changes in the expression of AQPs (Bodis et al., 2001). High concentration of CDCA also decreased the activity of the water channels, which is somewhat related to our previous findings on guinea pig pancreatic ducts, where $1 \mathrm{mM}$ CDCA strongly inhibited ductal $\mathrm{HCO}_{3}{ }^{-}$and thus fluid secretion (Venglovecz et al., 2008). The involvement of AQP1 in epithelial water movements has been described in various tissues, although there is still no consensus among researchers regarding the functional importance of this water channel (Marples, 2000). In cholangiocytes, the absence of AQP1 does not affect the fluid secretion (Mennone et al., 2002), although inhibition of the secretin-induced translocation of AQP1 reduces bile flow more than half (Marinelli et al., 1997, 1999). It has been also demonstrated that inhibition of AQPs by $\mathrm{HgCl}_{2}$ dose-dependently reduced the osmotically induced volume increase in these cells (Roberts et al., 1994). In the kidney, the absence of AQP1 dramatically decreased the urine concentrating ability of mice due to the impaired water permeability of the proximal tubule, limb of Henle and vasa recta (Ma et al., 1998; Schnermann et al., 1998; Pallone et al., 2000). The importance of AQP1 has been also highlighted in the brain where cerebrospinal fluid production decreased by fivefold in mice lacking AQP1 (Oshio et al., 2004).

In order to determine the role of AQP1 in pancreatic fluid secretion, we used AQP1 KO mice. No differences were observed in body weight and physical appearance between WT and KO mice, although the lifespan of AQP1 deficient mice was slightly lower. Using both in vitro and in vivo approaches, we found that fluid and $\mathrm{HCO}_{3}{ }^{-}$secretion significantly reduced in the absence of AQP1. These data are partly in contrast with previous observations demonstrating that defect in AQP1 expression cause only a small, but not significant decrease in the rate of stimulated pancreatic fluid secretion (Ma et al., 2001). Although this discrepancy can be explained by the different methods used for the measurement of pancreatic fluid. Nevertheless, other studies have found that decreased expression or function of AQP1 dramatically reduce ductal fluid secretion (Ko et al., 2002; Gabbi et al., 2008). Ko et al. (2002) have shown that luminal or basolateral administration of $\mathrm{HgCl}_{2}$ dose-dependently decrease the fluid secretory rate and osmotic water permeability of isolated pancreatic ducts. It has been also described that the defect in pancreatic fluid secretion in liver $X$ receptor $\beta$-deficient mice is related to the decreased expression of AQP1 in the pancreatic ducts of these mice (Gabbi et al., 2008).

In order to identify the mechanism by which AQP1 influences ductal $\mathrm{HCO}_{3}{ }^{-}$secretion, we investigated the relation of this channel with the CFTR $\mathrm{Cl}^{-}$channel. CFTR is a cAMP-activated $\mathrm{Cl}^{-}$channel, which primarily located at the apical membrane of epithelial cells and plays a crucial role in the maintenance of fluid homeostasis. Growing number of studies indicate that a molecular interaction exists between CFTR and certain AQP isoforms. It has been demonstrated that activation of CFTR by cAMP, increases the water permeability of the cells through the activation of AQP3 (Schreiber et al., 1999; Jourdain et al., 2014). 


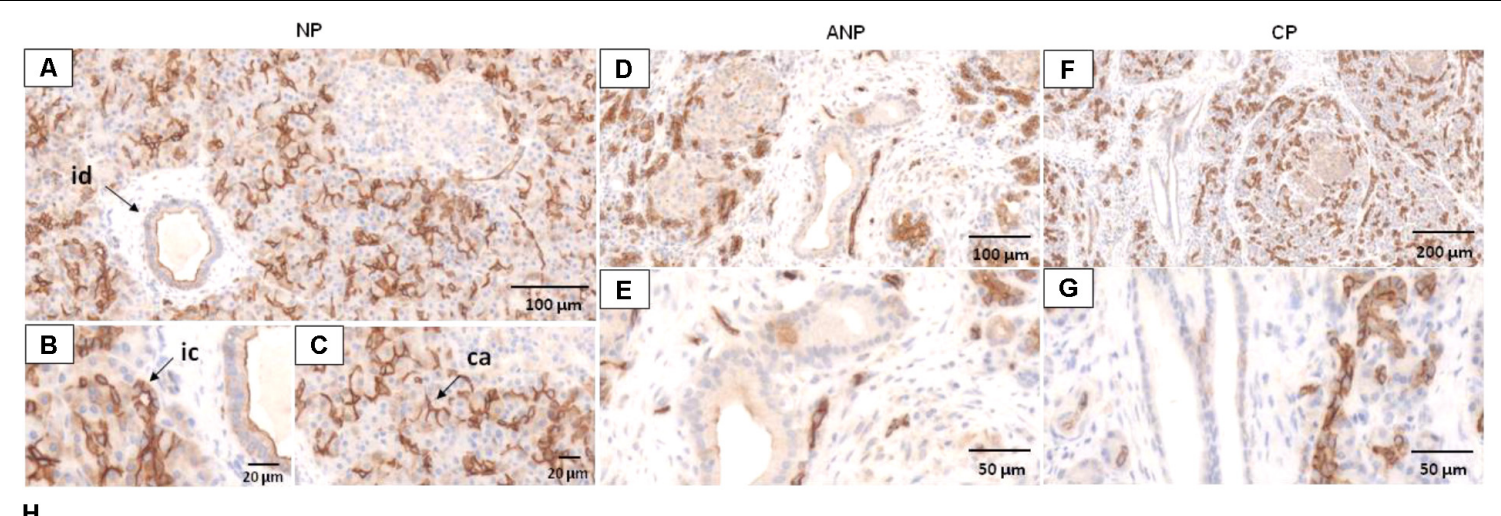

H

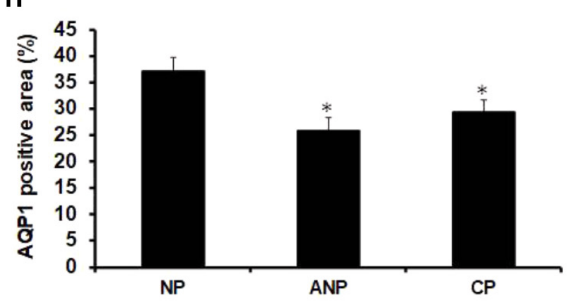

FIGURE 6 | Expression of AQP1 in patients with acute and chronic pancreatitis. Representative histological images show expression and localization of AQP1 in normal human pancreas (NP, A-C) acute necrotizing pancreatitis (ANP, D,E) and chronic pancreatitis (CP, F,G) samples. AQP1 immunoreactivity was detected in inter/intralobular and intercalated ducts, in the acinar and centroacinar cells of the normal pancreas. In contrast, expression of AQP1 strongly decreased in ANP and CP. id, interlobular duct; ic, intercalated duct; ca, centroacinar cells. (H) Intensity of diaminobenzidine staining was measured in pancreas samples by ImageJ software and expressed as percentage of total pancreatic area. ${ }^{*} p \leq 0.05$ vs. normal pancreas, $n=10-12$

The relation between AQPs and CFTR has been also confirmed in rat epididymis, where CFTR potentiates AQP9-mediated water permeability (Cheung et al., 2003). Moreover, a direct interaction between AQPs and CFTR has been also observed in Sertoli cells (Jesus et al., 2014a,b). Using double immunostaining we showed for the first time that AQP1 and CFTR are co-localized at the apical membrane of pancreatic ductal cells. Lack of CFTR significantly decreased the expression of AQP1, indicating that CFTR somehow controls the water permeability of ductal cells. Similar results have been found in respiratory epithelial cells, where in the presence of CFTR inhibitor or mutant CFTR the water permeability significantly decreased (Schreiber et al., 1999, 2000; Jourdain et al., 2014) which highlights the significance of this water channel in cystic fibrosis. In contrast, expression of CFTR does not depend on the presence of AQP1, since in the absence of this water channel, strong CFTR staining was detected. This result indicates that the decreased $\mathrm{HCO}_{3}{ }^{-}$secretion in the AQP1 KO mice is not due to the impaired expression of CFTR. Nevertheless, we cannot exclude that although the expression of CFTR did not change but the channel does not work correctly, however, further functional experiments are needed to confirm this hypothesis.

There is more and more evidence that impaired pancreatic fluid secretion plays role in the pathomechanism of pancreatitis (Hegyi and Rakonczay, 2010; Pallagi et al., 2014; Maleth et al., 2015). So in the next step, we investigated whether the lack of AQP1 has any effects on the progression of pancreatitis. The loss of AQP1 itself does not damage the pancreas and does not cause pancreatitis in mice, which assuming the compensating effect of
AQP5; however, induces a more severe disease progression. The involvement of AQP1 in the pathophysiology of pancreatitis has been already raised previously (Kitami et al., 2007). Kitami et al. (2007) have found that AQP1 expression decreased both in the ductal and acinar cells in a cerulein-induced pancreatitis model, which indicate that reduced levels of AQP1 may contribute to exocrine insufficiency. These findings are in accordance with our observation that expression of AQP1 decreased in the ductal and acinar cells of patients with AP or CP. The importance of AQP1 in the exocrine pancreas has been also confirmed by the fact that this water channel is abundantly expressed in the zymogen granules of acinar cells and plays an essential role in zymogen swelling and probably secretion (Cho et al., 2002). AQP12 is also expressed in the zymogen granule of acinar cells and huge amount of this isoform is present in the rough endoplasmic reticulum (Ohta et al., 2009). In the absence of AQP12, high concentration of cholecystokinin octapeptide makes the pancreas more susceptible to pancreatitis, presumably by the aberrant exocytosis of zymogen granules in these mice (Ohta et al., 2009). All of these previous observations indicate that AQP1 plays essential role both in ductal and acinar functions and we speculate that the absence of AQP1 makes the pancreas more sensitive in two ways: (1) ductal fluid secretion is not sufficient and (2) exocytosis of zymogen granules is impaired.

In this study, we provided an overview regarding the expression and role of AQP1 in the physiology and pathophysiology of the pancreas. Our data indicate that AQP1 interacts with the CFTR $\mathrm{Cl}^{-}$channel and takes part in the formation of pancreatic fluid. Moreover, we have found that 
AQP1 plays role in the pathology of pancreatitis. We hypothesize that absence of the channel makes the pancreas more sensitive to pancreatitis, probably due to the decreased pancreatic fluid and $\mathrm{HCO}_{3}{ }^{-}$secretion. Our novel findings not only help to understand the pathomechanism of pancreatitis better, but open up new therapeutic opportunities in the treatment of the disease.

\section{AUTHOR CONTRIBUTIONS}

PP performed microfluorimetric and videomicroscopy experiments. LK, ÁZ, and LP were involved in molecular biology experiments. AB, MS, and J-PK performed MRI and JM and ML interpreted the MRI pictures. Genotyping and breeding of AQP mice were done by EB and EG. Pancreatitis was induced by ZB. $\mathrm{KB}$ and $\mathrm{ET}$ did the immunostainings on the human and mice pancreatic samples, respectively, and quantified fluorescence intensity. ZR was involved in data interpretation and edited the manuscript. VV was involved in all of the above mentioned experiments, analyzed the data, and drafted the manuscript. $\mathrm{PH}$ supervised the project and edited the manuscript. All authors approved the final version of the manuscript.

\section{FUNDING}

This study was supported by the National Research, Development and Innovation Office (K109756 to VV, K116634 to $\mathrm{PH}$,

\section{REFERENCES}

Argent, B. E., Arkle, S., Cullen, M. J., and Green, R. (1986). Morphological, biochemical and secretory studies on rat pancreatic ducts maintained in tissueculture. Q. J. Exp. Physiol. 71, 633-648. doi: 10.1113/expphysiol.1986.sp003023

Bedford, J. J., Leader, J. P., and Walker, R. J. (2003). Aquaporin expression in normal human kidney and in renal disease. J. Am. Soc. Nephrol. 14, 2581-2587. doi: 10.1097/01.asn.0000089566.28106.f6

Bodis, B., Nagy, G., Nemeth, N., and Mozsik, G. (2001). Active water selective channels in the stomach: investigation of aquaporins after ethanol and capsaicin treatment in rats. J. Physiol. Paris 95, 271-275. doi: 10.1016/s0928-4257(01) 00037-37

Burghardt, B., Elkjaer, M. L., Kwon, T. H., Racz, G. Z., Varga, G., Steward, M. C., et al. (2003). Distribution of aquaporin water channels AQP1 and AQP5 in the ductal system of the human pancreas. Gut 52, 1008-1016. doi: 10.1136/gut.52. 7.1008

Cheung, K. H., Leung, C. T., Leung, G. P. H., and Wong, P. Y. D. (2003). Synergistic effects of cystic fibrosis transmembrane conductance regulator and aquaporin-9 in the rat epididymis. Biol. Reprod. 68, 1505-1510. doi: 10.1095/biolreprod.102. 010017

Cho, S. J., Sattar, A., Jeong, E. H., Satchi, M., Cho, J. A., Dash, S., et al. (2002). Aquaporin 1 regulates GTP-induced rapid gating of water in secretory vesicles. Proc. Natl. Acad. Sci. U.S.A. 99, 4720-4724. doi: 10.1073/pnas.07208 3499

Criddle, D. N., Murphy, J., Fistetto, G., Barrow, S., Tepikin, A. V., Neoptolemos, J. P., et al. (2006). Fatty acid ethyl esters cause pancreatic calcium toxicity via inositol trisphosphate receptors and loss of ATP synthesis. Gastroenterology 130, 781-793. doi: 10.1053/j.gastro.2005.12.031

Ding, S. P., Li, J. C., and Jin, C. (2003). A mouse model of severe acute pancreatitis induced with caerulein and lipopolysaccharide. World J. Gastroenterol. 9, 584589. doi: 10.3748/wjg.v9.i3.584 and K119938 to ZR), the Economic Development and Innovation Operative Programme Grants (GINOP-2.3.2-152016-00015 and GINOP-2.3.2-15-2016-00020 to PH), the National Research, Development and Innovation Office, by the Ministry of Human Capacities (EFOP 3.6.2-16-2017-00006 to ZR), by Bolyai Postdoctoral Fellowship of the Hungarian Academy of Sciences (HAS) to VV (00531/11/5), the HASUSZ Momentum Grant to PH (LP2014-10/2017), the TÁMOP 4.2.4.A/2-11-1-2012-0001 of the National Excellence Program, the Deutsche Forschungsgemeinschaft (DFG MA 4115/1-2/3, DFG SE 2702/2-1, and GRK 1947;A3), the Federal Ministry of Education and Research (BMBF GANI-MED 03IS2061A and BMBF 0314107, 01ZZ9603, 01ZZ0103, 01ZZ0403, 03ZIK012) and the European Union (EU-FP-7: EPC-TM), V-630-S-1502012/132/133 and PePPP center of excellence MV; ESF/14-BMA55-0045/16.

\section{SUPPLEMENTARY MATERIAL}

The Supplementary Material for this article can be found online at: https://www.frontiersin.org/articles/10.3389/fphys. 2018.00854/full\#supplementary-material

FIGURE S1 | Expression of AQP after various treatments. Capan-1 cells were treated with (A) glycochenodeoxycholic acid (GCDCA), (B) palmitoleic acid (POA), (C) palmitoleic acid ethyl ester and (D) ethanol (EtOH) for 6, 12, 24, and 48 h and the relative gene expression of AQP1 was investigated by real-time PCR. Data represent mean \pm SEM of three, independent experiments. ${ }^{*} p \leq 0.05$ vs. Control.

Direito, I., Paulino, J., Vigia, E., Brito, M. A., and Soveral, G. (2017). Differential expression of aquaporin-3 and aquaporin-5 in pancreatic ductal adenocarcinoma. J. Surg. Oncol. 115, 980-996. doi: 10.1002/jso.24605

Fernandez-Salazar, M. P., Pascua, P., Calvo, J. J., Lopez, M. A., Case, R. M., Steward, M. C., et al. (2004). Basolateral anion transport mechanisms underlying fluid secretion by mouse, rat and guinea-pig pancreatic ducts. J. Physiol. 556, 415-428. doi: 10.1113/jphysiol.2004.061762

Furuya, S., Naruse, S., Ko, S. B. H., Ishiguro, H., Yoshikawa, T., and Hayakawa, T. (2002). Distribution of aquaporin 1 in the rat pancreatic duct system examined with light- and election-microscopic immunohistochemistry. Cell Tissue Res. 308, 75-86. doi: 10.1007/s00441-002-0527-x

Gabbi, C., Kim, H. J., Hultenby, K., Bouton, D., Toresson, G., Warner, M., et al. (2008). Pancreatic exocrine insufficiency in LXR beta(-/-) mice is associated with a reduction in aquaporin-1 expression. Proc. Natl. Acad. Sci. U.S.A. 105, 15052-15057. doi: 10.1073/pnas.0808097105

Hegyi, P., Maleth, J., Venglovecz, V., and Rakonczay, Z. (2011a). Pancreatic ductal bicarbonate secretion: challenge of the acinar acid load. Front. Physiol. 2:36. doi: 10.3389/fphys.2011.00036

Hegyi, P., Pandol, S., Venglovecz, V., and Rakonczay, Z. Jr. (2011b). The acinarductal tango in the pathogenesis of acute pancreatitis. Gut 60, 544-552. doi: 10.1136/gut.2010.218461

Hegyi, P., and Rakonczay, Z. (2010). Insufficiency of electrolyte and fluid secretion by pancreatic ductal cells leads to increased patient risk for pancreatitis. Am. J. Gastroenterol. 105, 2119-2120. doi: 10.1038/ajg.2010.191

Hegyi, P., and Rakonczay, Z. (2015). The role of pancreatic ducts in the pathogenesis of acute pancreatitis. Pancreatology 15, S13-S17. doi: 10.1016/j. pan.2015.03.010

Hegyi, P., Rakonczay, Z., Gray, M. A., and Argent, B. E. (2004). Measurement of intracellular $\mathrm{pH}$ in pancreatic duct cells: a new method for calibrating the fluorescence data. Pancreas 28, 427-434. doi: 10.1097/00006676-200405000200405012 
Huang, X. W., Huang, L., and Shao, M. H. (2017). Aquaporin 3 facilitates tumor growth in pancreatic cancer by modulating mTOR signaling. Biochem. Biophys. Res. Commun. 486, 1097-1102. doi: 10.1016/j.bbrc.2017.03.168

Hurley, P. T., Ferguson, C. J., Kwon, T. H., Andersen, M. L. E., Norman, A. G., Steward, M. C., et al. (2001). Expression and immunolocalization of aquaporin water channels in rat exocrine pancreas. Am. J. Physiol. Gastrointestinal Liver Physiol. 280, G701-G709. doi: 10.1152/ajpgi.2001.280.4.G701

Itoh, T., Rai, T., Kuwahara, M., Ko, S. B. H., Uchida, S., Sasaki, S., et al. (2005). Identification of a novel aquaporin, AQP12, expressed in pancreatic acinar cells. Biochem. Biophys. Res. Commun. 330, 832-838. doi: 10.1016/j.bbrc.2005.03.046

Jesus, T. T., Bernardino, R. L., Martins, A. D., Sa, R., Sousa, M., Alves, M. G., et al. (2014a). Aquaporin-4 as a molecular partner of cystic fibrosis transmembrane conductance regulator in rat Sertoli cells. Biochem. Biophys. Res. Commun. 446, 1017-1021. doi: 10.1016/j.bbrc.2014.03.046

Jesus, T. T., Bernardino, R. L., Martins, A. D., Sa, R., Sousa, M., Alves, M. G., et al. (2014b). Aquaporin-9 is expressed in rat sertoli cells and interacts with the cystic fibrosis transmembrane conductance regulator. IUBMB Life 66, 639-644. doi: 10.1002/iub.1312

Jourdain, P., Becq, F., Lengacher, S., Boinot, C., Magistretti, P. J., and Marquet, P. (2014). The human CFTR protein expressed in CHO cells activates aquaporin-3 in a cAMP-dependent pathway: study by digital holographic microscopy. J. Cell Sci. 127, 546-556. doi: 10.1242/jcs.133629

Judak, L., Hegyi, P., Rakonczay, Z. Jr., Maleth, J., Gray, M. A. and Venglovecz, V. (2014). Ethanol and its non-oxidative metabolites profoundly inhibit CFTR function in pancreatic epithelial cells which is prevented by ATP supplementation. Pflugers Arch. 466, 549-562. doi: 10.1007/s00424-013-1333-x

Kitami, C.-E., Koyama, Y., Nishino, H., Kurosaki, I., Iiai, T., and Hatakeyama, K. (2007). Dynamics of aquaporin 1 and aquaporin 8 in the pancreas: an experimental study with a caerulein-induced pancreatitis model. Acta Med. Biol. 55, 91-101.

Ko, S. B. H., Naruse, S., Kitagawa, M., Ishiguro, H., Furuya, S., Mizuno, N., et al. (2002). Aquaporins in rat pancreatic interlobular ducts. Am. J. Physiol. Gastrointestinal Liver Physiol. 282, G324-G331. doi: 10.1152/ajpgi.00198.2001

Kui, B., Balla, Z., Vasas, B., Vegh, E. T., Pallagi, P., Kormanyos, E. S., et al. (2015). New insights into the methodology of L-arginine-induced acute pancreatitis. PLoS One 10:e0117588. doi: 10.1371/journal.pone.0117588

Levin, M. H., Sullivan, S., Nielson, D., Yang, B. X., Finkbeiner, W. E., and Verkman, A. S. (2006). Hypertonic saline therapy in cystic fibrosis: evidence against the proposed mechanism involving aquaporins. J. Biol. Chem. 281, 25803-25812. doi: 10.1074/jbc.M604332200

Ma, T. H., Jayaraman, S., Wang, K. S., Song, Y. L., Yang, B. X., Li, J., et al. (2001). Defective dietary fat processing in transgenic mice lacking aquaporin-1 water channels. Am. J. Physiol. Cell Physiol. 280, C126-C134. doi:/10.1152/ajpcell. 2001.280.1.C126

Ma, T. H., Yang, B. X., Gillespie, A., Carlson, E. J., Epstein, C. J., and Verkman, A. S. (1998). Severely impaired urinary concentrating ability in transgenic mice lacking aquaporin-1 water channels. J. Biol. Chem. 273, 4296-4299. doi: $10.1074 /$ jbc. 273.8 .4296

Madeira, A., Moura, T. F., and Soveral, G. (2015). Aquaglyceroporins: implications in adipose biology and obesity. Cell. Mol. Life Sci. 72, 759-771. doi: 10.1007/s00018-014-1773-2

Maleth, J., Balazs, A., Pallagi, P., Balla, Z., Kui, B., Katona, M., et al. (2015). Alcohol disrupts levels and function of the cystic fibrosis transmembrane conductance regulator to promote development of pancreatitis. Gastroenterology 148, 427-439. doi: 10.1053/j.gastro.2014.11.002

Marinelli, R. A., Pham, L., Agre, P., and LaRusso, N. F. (1997). Secretin promotes osmotic water transport in rat cholangiocytes by increasing aquaporin-1 water channels in plasma membrane: evidence for a secretin-induced vesicular translocation of aquaporin-1. J. Biol. Chem. 272, 12984-12988. doi: 10.1074/ jbc.272.20.12984

Marinelli, R. A., Tietz, P. S., Pham, L. D., Rueckert, L., Agre, P., and LaRusso, N. F. (1999). Secretin induces the apical insertion of aquaporin-1 water channels in rat cholangiocytes. Am. J. Physiol. 276, G280-G286. doi: 10.1152/ajpgi.1999. 276.1.G280

Marples, D. (2000). Water channels: who needs them anyway? Lancet 355, 1571-1572. doi: 10.1016/s0140-6736(00)02209-1

Mennone, A., Verkman, A. S., and Boyer, J. L. (2002). Unimpaired osmotic water permeability and fluid secretion in bile duct epithelia of AQP1 null mice. Am.
J. Physiol. Gastrointestinal Liver Physiol. 283, G739-G746. doi: 10.1152/ajpgi. 00540.2001

Muili, K. A., Wang, D., Orabi, A. I., Sarwar, S., Luo, Y., Javed, T. A., et al. (2013). Bile acids induce pancreatic acinar cell injury and pancreatitis by activating calcineurin. J. Biol. Chem. 288, 570-580. doi: 10.1074/jbc.M112.428896

Niederau, C., Ferrell, L. D., and Grendell, J. H. (1985). Caerulein-induced acute necrotizing pancreatitis in mice: protective effects of proglumide, benzotript, and secretin. Gastroenterology 88(5 Pt 1), 1192-1204. doi: 10.1016/S00165085(85)80079-2

Novak, I., and Greger, R. (1988). Electrophysiological study of transport-systems in isolated perfused pancreatic ducts - properties of the basolateral membrane. Pflugers Arch. 411, 58-68. doi: 10.1007/bf00581647

Ohta, E., Itoh, T., Nemoto, T., Kumagai, J., Ko, S. B. H., Ishibashi, K., et al. (2009). Pancreas-specific aquaporin 12 null mice showed increased susceptibility to caerulein-induced acute pancreatitis. Am. J. Physiol. Cell Physiol. 297, C1368C1378. doi: 10.1152/ajpcell.00117.2009

Oshio, K., Watanabe, H., Song, Y., Verkman, A. S., and Manley, G. T. (2004). Reduced cerebrospinal fluid production and intracranial pressure in mice lacking choroid plexus water channel Aquaporin-1. FASEB J. 18, 76-78. doi: 10.1096/fj.04-1711fje

Pallagi, P., Balla, Z., Singh, A. K., Dosa, S., Ivanyi, B., Kukor, Z., et al. (2014). The role of pancreatic ductal secretion in protection against acute pancreatitis in mice*. Crit. Care Med. 42, e177-e188. doi: 10.1097/CCM.0000000000000101

Pallagi, P., Hegyi, P., and Rakonczay, Z. (2015). The physiology and pathophysiology of pancreatic ductal secretion the background for clinicians. Pancreas 44, 1211-1233. doi: 10.1097/mpa.0000000000000421

Pallagi, P., Venglovecz, V., Rakonczay, Z., Borka, K., Korompay, A., Ozsvari, B., et al. (2011). Trypsin reduces pancreatic ductal bicarbonate secretion by inhibiting cftr cl-channels and luminal anion exchangers. Gastroenterology 141, 2228-2239. doi: 10.1053/j.gastro.2011.08.039

Pallone, T. L., Edwards, A., Ma, T. H., Silldorff, E. P., and Verkman, A. S. (2000). Requirement of aquaporin-1 for NaCl-driven water transport across descending vasa recta. J. Clin. Invest. 105, 215-222. doi: 10.1172/jci8214

Parniczky, A., Kui, B., Szentesi, A., Balazs, A., Szucs, A., Mosztbacher, D., et al. (2016). Prospective, multicentre, nationwide clinical data from 600 cases of acute pancreatitis. PLoS One 11:e0165309. doi: 10.1371/journal.pone. 0165309

Pascua, P., Garcia, M., Fernandez-Salazar, M. P., Hernandez-Lorenzo, M. P., Calvo, J. J., Colledge, W. H., et al. (2009). Ducts isolated from the pancreas of CFTRnull mice secrete fluid. Pflugers Arch. 459, 203-214. doi: 10.1007/s00424-0090704-9

Preston, G. M., Carroll, T. P., Guggino, W. B., and Agre, P. (1992). Appearance of water channels in xenopus oocytes expressing red-cell chip28 protein. Science 256, 385-387. doi: 10.1126/science.256.5055.385

Ribatti, D., Ranieri, G., Annese, T., and Nico, B. (2014). Aquaporins in cancer. Biochim. Biophys. Acta 1840, 1550-1553. doi: 10.1016/j.bbagen.2013.09.025

Roberts, S. K., Yano, M., Ueno, Y., Pham, L., Alpini, G., Agre, P., et al. (1994). Cholangiocytes express the aquaporin chip and transport water via a channel-mediated mechanism. Proc. Natl. Acad. Sci. U.S.A. 91, 13009-13013. doi: 10.1073/pnas.91.26.13009

Rodriguez, A., Catalan, V., Gomez-Ambrosi, J., and Fruhbeck, G. (2011). Aquaglyceroporins serve as metabolic gateways in adiposity and insulin resistance control. Cell Cycle 10, 1548-1556. doi: 10.4161/cc.10.10. 15672

Schnermann, J., Chou, C. L., Ma, T. H., Traynor, T., Knepper, M. A., and Verkman, A. S. (1998). Defective proximal tubular fluid reabsorption in transgenic aquaporin-1 null mice. Proc. Natl. Acad. Sci. U.S.A. 95, 9660-9664. doi: 10.1073/ pnas. 95.16 .9660

Schreiber, R., Nitschke, R., Greger, R., and Kunzelmann, K. (1999). The cystic fibrosis transmembrane conductance regulator activates aquaporin 3 in airway epithelial cells. J. Biol. Chem. 274, 11811-11816. doi: 10.1074/jbc.274.17. 11811

Schreiber, R., Pavenstadt, H., Greger, R., and Kunzelmann, K. (2000). Aquaporin 3 cloned from Xenopus laevis is regulated by the cystic fibrosis transmembrane conductance regulator. FEBS Lett. 475, 291-295. doi: 10.1016/s0014-5793(00) 01689-6

Steinfeld, S., Cogan, E., King, L. S., Agre, P., Kiss, R., and Delporte, C. (2001). Abnormal distribution of aquaporin-5 water channel protein in salivary glands 
from Sjogren's syndrome patients. Lab. Invest. 81, 143-148. doi: 10.1038/ labinvest.3780221

Steward, M. C., Ishiguro, H., and Case, R. M. (2005). Mechanisms of bicarbonate secretion in the pancreatic duct. Annu. Rev. Physiol. 67, 377-409. doi: 10.1146/ annurev.physiol.67.031103.153247

Szucs, A., Marjai, T., Szentesi, A., Farkas, N., Parniczky, A., Nagy, G., et al. (2017). Chronic pancreatitis: multicentre prospective data collection and analysis by the Hungarian Pancreatic Study Group. PLoS One 12:e0171420. doi: 10.1371/ journal.pone.0171420

Takacs, T., Rosztoczy, A., Maleth, J., Rakonczay, Z., and Hegyi, P. (2013). Intraductal acidosis in acute biliary pancreatitis. Pancreatology 13, 333-335. doi: 10.1016/j.pan.2013.05.011

Tani, T., Koyama, Y., Nihei, K., Hatakeyama, S., Ohshiro, K., Yoshida, Y., et al. (2001). Immunolocalization of aquaporin-8 in rat digestive organs and testis. Arch. Histol. Cytol. 64, 159-168. doi: 10.1679/aohc. 64.159

Thomas, J. A., Buchsbaum, R. N., Zimniak, A., and Racker, E. (1979). Intracellular ph measurements in ehrlich ascites tumor-cells utilizing spectroscopic probes generated insitu. Biochemistry 18, 2210-2218. doi: 10.1021/bi0057 $8 \mathrm{a} 012$

Tradtrantip, L., Tajima, M., Li, L., and Verkman, A. S. (2009). Aquaporin water channels in transepithelial fluid transport. J. Med. Invest. 56(Suppl.), 179-184. doi: $10.2152 /$ jmi.56.179

Tsubota, K., Hirai, S., King, L. S., Agre, P., and Ishida, N. (2001). Defective cellular trafficking of lacrimal gland aquaporin-5 in Sjogren's syndrome. Lancet 357, 688-689. doi: 10.1016/s0140-6736(00)04140-4
Venglovecz, V., Hegyi, P., Rakonczay, Z. Jr., Tiszlavicz, L., Nardi, A., Grunnet, M., et al. (2011). Pathophysiological relevance of apical large-conductance $\mathrm{Ca}(2)+-$ activated potassium channels in pancreatic duct epithelial cells. Gut 60, 361369. doi: 10.1136/gut.2010.214213

Venglovecz, V., Rakonczay, Z., Ozsvari, B., Takacs, T., Lonovics, J., Varro, A., et al. (2008). Effects of bile acids on pancreatic ductal bicarbonate secretion in guinea pig. Gut 57, 1102-1112. doi: 10.1136/gut.2007.134361

Yde, J., Keely, S., Wu, Q., Borg, J. F., Lajczak, N., O’Dwyer, A., et al. (2016). Characterization of AQPs in mouse, rat, and human colon and their selective regulation by bile acids. Front. Nutr. 3:46. doi: 10.3389/fnut.2016.00046

Zhu, C., Chen, Z., and Jiang, Z. Y. (2016). Expression, distribution and role of aquaporin water channels in human and animal stomach and intestines. Int. J. Mol. Sci. 17:1399. doi: 10.3390/ijms17091399

Conflict of Interest Statement: The authors declare that the research was conducted in the absence of any commercial or financial relationships that could be construed as a potential conflict of interest.

Copyright (c) 2018 Venglovecz, Pallagi, Kemény, Balázs, Balla, Becskeházi, Gál, Tóth, Zvara, Puskás, Borka, Sendler, Lerch, Mayerle, Kühn, Rakonczay and Hegyi. This is an open-access article distributed under the terms of the Creative Commons Attribution License (CC BY). The use, distribution or reproduction in other forums is permitted, provided the original author(s) and the copyright owner(s) are credited and that the original publication in this journal is cited, in accordance with accepted academic practice. No use, distribution or reproduction is permitted which does not comply with these terms. 


\section{OPEN ACCESS}

Edited by:

Atsushi Masamune,

Tohoku University, Japan

Reviewed by:

Matthias J. Bahr,

Sana Kliniken Lübeck, Germany

Tomohiro Watanabe,

Kindai University, Japan

*Correspondence:

Peter Hegyi

hegyi.peter@pte.hu

Andras Garami

andras.garami@aok.pte.hu

tThese authors have contributed equally to this work

Specialty section: This article was submitted to

Gastrointestinal Sciences,

a section of the journal

Frontiers in Physiology

Received: 07 April 2018 Accepted: 07 September 2018

Published: 01 October 2018

Citation:

Rumbus Z, Toth E, Poto L, Vincze A, Veres G, Czako L, Olah E, Marta K,

Miko A, Rakonczay Z Jr, Balla Z,

Kaszaki J, Foldesi I, Maleth J, Hegyi P

and Garami A (2018) Bidirectional

Relationship Between Reduced Blood

$\mathrm{pH}$ and Acute Pancreatitis: A

Translational Study of Their Noxious

Combination. Front. Physiol. 9:1360.

doi: 10.3389/fphys.2018.01360

\section{Bidirectional Relationship Between Reduced Blood pH and Acute Pancreatitis: A Translational Study of Their Noxious Combination}

\begin{abstract}
Zoltan Rumbus ${ }^{1 \dagger}$, Emese Toth ${ }^{2,3+}$, Laszlo Poto ${ }^{4}$, Aron Vincze ${ }^{5}$, Gabor Veres ${ }^{6}$, Laszlo Czako ${ }^{3}$, Emoke Olah ${ }^{1}$, Katalin Marta ${ }^{1,7}$, Alexandra Miko ${ }^{1,7}$, Zoltan Rakonczay Jr. ${ }^{8}$, Zsolt Balla ${ }^{8}$, Jozsef Kaszaki ${ }^{9}$, Imre Foldesi ${ }^{10}$, Jozsef Maleth ${ }^{3,11}$, Peter Hegyi ${ }^{1,2,7 *}$ and Andras Garami ${ }^{1 *}$
\end{abstract}

\footnotetext{
${ }^{1}$ Institute for Translational Medicine, Medical School, University of Pecs, Pecs, Hungary, ${ }^{2}$ Momentum Gastroenterology Multidisciplinary Research Group, Hungarian Academy of Sciences-University of Szeged, Szeged, Hungary, ${ }^{3}$ First Department of Medicine, University of Szeged, Szeged, Hungary, ${ }^{4}$ Institute of Bioanalysis, Medical School, University of Pecs, Pecs, Hungary, ${ }^{5}$ Department of Gastroenterology, First Department of Medicine, University of Pecs, Pecs, Hungary, ${ }^{6}$ First Department of Pediatrics, Semmelweis University, Budapest, Hungary, ${ }^{7}$ Department of Translational Medicine, First Department of Medicine, University of Pecs, Pecs, Hungary, ${ }^{8}$ Department of Pathophysiology, University of Szeged, Szeged, Hungary, ${ }^{9}$ Institute of Surgical Research, University of Szeged, Szeged, Hungary, ${ }^{10}$ Department of Laboratory Medicine, University of Szeged, Szeged, Hungary, ${ }^{11}$ Momentum Epithel Cell Signaling and Secretion Research Group, Hungarian Academy of Sciences-University of Szeged, Szeged, Hungary
}

Acute pancreatitis (AP) is often accompanied by alterations in the acid-base balance, but how blood $\mathrm{pH}$ influences the outcome of AP is largely unknown. We studied the association between blood $\mathrm{pH}$ and the outcome of AP with meta-analysis of clinical trials, and aimed to discover the causative relationship between blood $\mathrm{pH}$ and $\mathrm{AP}$ in animal models. PubMed, EMBASE, and Cochrane Controlled Trials Registry databases were searched from inception to January 2017. Human studies reporting systemic pH status and outcomes (mortality rate, severity scores, and length of hospital stay) of patient groups with AP were included in the analyses. We developed a new mouse model of chronic metabolic acidosis (MA) and induced mild or severe AP in the mice. Besides laboratory blood testing, the extent of pancreatic edema, necrosis, and leukocyte infiltration were assessed in tissue sections of the mice. Thirteen studies reported sufficient data in patient groups with AP $(n=2,311)$. Meta-analysis revealed markedly higher mortality, elevated severity scores, and longer hospital stay in AP patients with lower blood $\mathrm{pH}$ or base excess ( $P<0.001$ for all studied outcomes). Meta-regression analysis showed significant negative correlation between blood $\mathrm{pH}$ and mortality in severe AP. In our mouse model, pre-existing MA deteriorated the pancreatic damage in mild and severe AP and, vice versa, severe AP further decreased the blood $\mathrm{pH}$ of mice with MA. In conclusion, MA worsens the outcome of AP, while severe AP augments the decrease of blood $\mathrm{pH}$. The discovery of this vicious metabolic cycle opens up new therapeutic possibilities in AP.

Keywords: experimental pancreatitis, acidosis, acid-base balance, meta-analysis, mortality 


\section{INTRODUCTION}

Acute pancreatitis (AP) is one of the most frequent gastrointestinal causes of hospitalization with significant morbidity and mortality in the US (Yadav and Lowenfels, 2013; Parniczky et al., 2016). Although the mortality rate in mild and moderate AP is low, this value is still unacceptably high (30\%) in its severe form (Parniczky et al., 2016). Since no specific therapy is available, only prompt and accurate interventions, such as aggressive fluid therapy can be beneficial (Vinish et al., 2017).

An important function of the pancreas is bicarbonate production, which is required to maintain its constant "milieu intérieur," thereby to prevent premature activation of pancreatic proteases (Pallagi et al., 2011, 2015; Hegyi and Petersen, 2013). When pancreatic bicarbonate production is challenged by local or systemic acid load (i.e., metabolic acidosis, MA), the resulting lower $\mathrm{pH}$ can facilitate pancreatic enzyme activation and deteriorate cell damage (Reed et al., 2011). Furthermore, injection of acidic contrast solution either into the pancreatic duct or into the vein significantly increased the severity of AP in rats (Noble et al., 2008; Bhoomagoud et al., 2009). Beside an external acid load, the pancreatic $\mathrm{pH}$ balance can also be compromised by tissue injury such as AP, which can lead to acidification of local tissues, thus deteriorate cell damage (Behrendorff et al., 2010). The luminal $\mathrm{pH}$ of the main pancreatic duct was also lower in human patients with AP compared to controls (Takacs et al., 2013), suggesting that the development of AP is accompanied by a reduction of local $\mathrm{pH}$. Multiple mechanisms have been implicated in AP which can lead to MA, including direct mechanisms such as the loss of bicarbonate-rich pancreatic juice via pancreatic fistula or drainage (Rice et al., 2014), as well as indirect ones through lactic acidosis which can sequentially occur in AP due to shock, sepsis, cardiovascular failure, or upper gastrointestinal bleeding (Zhan et al., 2015). However, the interaction between AP and systemic $\mathrm{pH}$ is still not fully clarified.

Acidosis is often considered as a marker of disease severity, viz., a by-product of systemic dysregulation, and as such it is a proven prognostic factor in the assessment of critically ill patients (Vincent and Moreno, 2010). Despite the fact that scoring systems, which are used to help the diagnosis and the assessment of the progression of AP, include the changes in systemic $\mathrm{pH}$ balance of the patients (e.g., Acute Physiology and Chronic Health Evaluation, APACHE II and Ranson scores), clinical trials aiming to reveal a correlation between the acidbase status and the outcome of AP are scarce. To our knowledge, the sole published human study, which aimed to directly answer this question showed that changes in the parameters of systemic acid-base status can predict mortality in AP (Sharma et al., 2014). On the contrary, the necessity of arterial blood gas sampling was questioned in patients with AP in another human study (Ward

Abbreviations: AP, acute pancreatitis; APACHE, acute physiology and chronic health evaluation; CI, confidence interval; CRP, C-reactive protein; ES, estimated logit mortality rate; IL, interleukin; i.p., intraperitoneal(ly); LOS, length of hospital stay; MA, metabolic acidosis; MAP and SAP, mild and severe acute pancreatitis, respectively; SEM, standard error of mean; SMD, standardized mean difference; TNF, tumor necrosis factor. et al., 2008). With regards to the results obtained in experimental animals, a detailed analysis of the correlation between systemic $\mathrm{pH}$ and the outcome of AP would be of utmost importance, because it could establish blood $\mathrm{pH}$ as a predictor of the severity and the outcome of the disease and, arguably, identify acidosis as a therapeutic target in AP.

In the present study, by using a dual, translational approach, we have discovered a vicious, bidirectional interaction between blood $\mathrm{pH}$ and the outcome of AP. Based on our discovery, the possibility of new therapeutic approaches in AP can be suggested.

\section{MATERIALS AND METHODS}

\section{Study Design 1: Meta-Analysis of Clinical Trials}

\section{Search Strategy}

Our meta-analysis was conducted in accordance with the guidelines of the Preferred Reporting Items for Systematic Reviews and Meta-Analysis Protocols (Moher et al., 2009) (Supplementary Table 1), similarly as in our recent study (Olah et al., 2018). The analysis was based on the Patients, Intervention (or indicator), Comparison, Outcome (PICO) model: in patients with AP, we aimed to assess the predictive role of the change in $\mathrm{pH}$ status (as assessed by blood $\mathrm{pH}$, bicarbonate concentration, base excess, or base deficit) on disease severity (indicated by clinical scores), length of hospital stay (LOS), and mortality ratio. This meta-analysis has been registered with PROSPERO (CRD42017055396).

A search in the PubMed, EMBASE, and Cochrane Controlled Trials Registry databases was performed from inception to January 2017 using the following terms: "pancreatitis AND (mortality OR survival OR severity) AND ("arterial $\mathrm{pH}$ " OR "blood $\mathrm{pH}$ " OR "systemic $\mathrm{pH}$ " OR "base deficit" OR "base excess" OR bicarbonate OR HCO3- OR "anion gap" OR acidosis OR alkalosis OR acid-base)." We restricted our search to original human studies published in English without time period limitations. A manual search of the reference lists of relevant fulltext articles was conducted to identify further potentially eligible articles. The search was conducted separately by two authors ( $\mathrm{ZRu}, \mathrm{AG}$ ), who also assessed study eligibility and extracted data from the selected studies independently. Disagreements were resolved by consensus with the help of a third party $(\mathrm{PH})$.

\section{Study Selection and Data Extraction}

The titles and abstracts of the publications from the literature search were screened and the full text of potentially eligible articles was obtained. We included studies in which blood $\mathrm{pH}$ or a related parameter (e.g., base excess, base deficit, or bicarbonate) and severity scores or LOS or mortality ratios were reported for the same group(s) of patients with AP. From all included articles we extracted the sample size, the reported mean $\mathrm{pH}$ or its related parameter for the studied patient groups with the corresponding standard error (SEM) or deviation, as well as the severity score, LOS, and mortality ratio within the group. To analyze the influence of the change in acid-base status on the severity and the outcome of AP, in each study we assigned the patient groups as a lower $\mathrm{pH}$ group and as a higher $\mathrm{pH}$ group, 
irrespective from the original basis for grouping used by the authors of the study.

\section{Outcomes of Interest}

We used mortality ratio of the AP patients groups as the primary outcome. Regarding secondary outcomes, we used two commonly applied severity indices (i.e., APACHE II and Ranson scores) and the LOS.

\section{Quality Assessment}

We assessed the quality of each study included in the metaanalysis by using the Newcastle-Ottawa Scale (Wells et al., 2000; Supplementary Table 2).

\section{Statistical Analysis}

We used logit transformation of event rates for mortality ratios and standardized mean difference (SMD) for LOS and severity scores as the effect size data. The secondary outcomes were compared between the lower and higher $\mathrm{pH}$ groups (see above) within each study, and then the estimated pooled mean values were calculated. The relevant studies were compared with standard meta-analysis tools (e.g., forest plot) in case of each outcome.

Between-study heterogeneity was assessed by $\mathrm{I}^{2}$ statistical test, where $\mathrm{I}^{2}$ is the proportion of total variation attributable to between-study variability (an $\mathrm{I}^{2}$ value of more than 50 was considered as indication of considerable statistical heterogeneity). The selection of patients, study design, and the used methods showed variability among the studies included in our analyses, which also resulted in statistical heterogeneity. Since the lack of statistical significant results on these heterogeneity tests could be also due to the lack of power because of the small number of studies eligible for the analyses, we used the random effect model in case of each forest plot, similarly to our earlier meta-analysis (Rumbus et al., 2017). Publication bias was assessed by funnel-plot analysis, Egger's test and Duval and Tweedie trim and fill method (Supplementary Figures 1-5). Publication bias plots were used to assess whether studies with small sizes could have been missed in our analyses, however, due to the design of these tests they do not allow to firmly rule out the possibility that some papers missed the inclusion criteria of our search.

As a different statistical approach to reveal a correlation between systemic $\mathrm{pH}$ and mortality in moderate and severe forms of AP, we performed meta-regression analysis of those studies in which both blood $\mathrm{pH}$ and mortality rate were reported within the same patient group. The meta-analyses were performed with Comprehensive Meta-Analysis (version 3.3; Biostat, Inc., Engelwood, MJ, USA) and Stata (version 11.1; StataCorp, College Station, TX, USA) software.

\section{Study Design 2: Experimental Procedures Animals}

The experiments were performed in 40 female $\mathrm{FVB} / \mathrm{N}$ mice (Charles Rivers Laboratories, Wilmington, MA, USA). This commercially available, multipurpose mouse strain is characterized by excellent reproductive performance and it was repeatedly used by our group to study the mechanisms of AP (Kui et al., 2015; Maleth et al., 2015). The mice were housed in standard plastic cages kept in a room with an ambient temperature of $24^{\circ} \mathrm{C}$ on a 12 -h light-dark cycle in the animal facility of the First Department of Medicine at the University of Szeged. The mice were allowed free access to water and standard laboratory chow for rodents (Biofarm, Zagyvaszanto, Hungary).

\section{Ethics}

All experiments were approved by the Institutional Animal Care and Use Committee of the University of Szeged and also by an independent committee assembled by national authorities (XII/3773/2012.). All experiments were conducted in compliance with the European Union Directive (2010/63/EU) and the Hungarian government decree (40/2013, II.14.).

\section{Experimental Modeling of Chronic MA in Mice}

To develop a mouse model of chronic MA, the mice were randomly divided into the following 4 groups for a 12-day treatment: (i) ammonium chloride $\left(\mathrm{NH}_{4} \mathrm{Cl}\right)$ administration with drinking water $(8.2 \pm 0.5 \mathrm{ml} /$ day/mouse $)$ as reported in earlier studies (Galicek et al., 1981; Nowik et al., 2010); (ii) intraperitoneal (i.p.) injections of $\mathrm{NH}_{4} \mathrm{Cl}(0.5 \mathrm{ml}, 0.28 \mathrm{M})$ on days 1 and 6; (iii) administration of $\mathrm{NH}_{4} \mathrm{Cl}$ with drinking water (as in group 1) and i.p. injections (as in group 2); and (iv) controls, receiving $\mathrm{NH}_{4} \mathrm{Cl}$-free tap water and 2 i.p. injections of saline on days 1 and 6 .

\section{Experimental Modeling of AP}

Two different types of AP were used in this study. Mild acute pancreatitis (MAP) was induced by alcohol and fatty acid as described earlier (Huang et al., 2014; Maleth et al., 2015). Severe acute pancreatitis (SAP) was induced by the injections of cerulein $(50 \mu \mathrm{g} / \mathrm{kg}$, i.p.) at start time, and then at every hour for $9 \mathrm{~h}$ (Mareninova et al., 2006). In the chronic MA model, MAP and SAP were induced on day 12 of the acidifying treatment.

\section{Laboratory Measurements}

Animals were euthanized by i.p. injection of sodium pentobarbital $(50 \mathrm{mg} / \mathrm{kg})$. Blood samples were collected by cardiac puncture. Serum amylase activity was measured by using a colorimetric kinetic method (Diagnosticum, Budapest, Hungary). Serum concentrations of creatinine and glucose as well as urea concentration in urine were measured with commercially available laboratory kits (Institute of Laboratory Medicine, University of Szeged). Arterial blood samples were collected in sealed plastic capillaries $(170 \mu \mathrm{l})$, which were previously treated with lithium and heparin. Analysis of the arterial blood samples was performed by a blood gas analyzer (Cobas b221 system; Roche Ltd., Basel, Switzerland) within $1 \mathrm{~min}$ after blood collection at room temperature $\left(22^{\circ} \mathrm{C}\right)$.

\section{Histology}

Histological evaluations were performed as described earlier (Kui et al., 2015). In brief, the extent of pancreatic edema (0: none; 1 : patchy interlobular; 2: diffuse interlobular; 3: diffuse interlobular and intraacinar), necrosis (\%), and leukocyte infiltration (0: none; 1: rare patchy interlobular; 2: patchy interlobular; 3: diffuse 
interlobular; 4: diffuse interlobular and intraacinar) were assessed in pancreatic tissue sections stained with haematoxylin and eosin under a light microscope (Zeiss Axio scope A1 microscope) at 40x magnification by an investigator who was expert in pancreas histology, however blinded to the animal's treatment group. The percentage of acinar cell necrosis was evaluated by ImageJ software (NIH, Bethesda, MD, USA).

\section{Statistical Analysis}

Data were compared by one-way ANOVA followed by HolmSidak test, two-way ANOVA followed by Fischer Least Significant Difference test, or two-tailed Student's $t$ test, as appropriate. SPSS 23.0 (IBM, Armonk, NY, USA) and Microsoft Excel (Microsoft Corporation, Redmond, WA, USA) software was used for statistical analysis. The effects were considered significant when $P<0.05$. In the experimental part of the study, data are reported in the Mean \pm SEM format.

\section{RESULTS}

\section{Meta-Analysis}

\section{Study Selection}

The flow chart of the study selection is presented in Figure 1. Until January 2017 the electronic literature search identified altogether 1,076 studies from the PubMed, EMBASE, and Cochrane databases. After enabling filters for human studies and English language and removal of duplicates, 793 articles remained, which were screened on title and abstract for inclusion criteria. Full texts of the remaining 122 articles were reviewed in detail. In 109 studies $\mathrm{pH}$ parameters or outcomes were not suitably reported in the patients with AP, therefore these were also excluded. As a result, 13 full-text publications were found eligible for statistical analysis which included data from a total of 2,311 patients (Ranson et al., 1976; Nair et al., 2000; Eachempati et al., 2002; Zhu et al., 2003; Kaya et al., 2007; Keskinen et al., 2007; Pupelis et al., 2007; De Campos et al., 2008; Shinzeki et al., 2008; Lei et al., 2013; Sharma et al., 2014; Zhan et al., 2015; Shen et al., 2016). The characteristics of these studies are summarized in Supplementary Table 3.

\section{Reduction of Blood pH Is Associated With Higher Mortality Rate in AP}

First, we investigated the association between systemic (blood) $\mathrm{pH}$ status and our strongest endpoint, viz., the mortality. Our meta-analysis revealed a logit event rate of -0.09 (95\% CI, -0.79 , $0.61)$, corresponding to an average mortality rate of $51.0 \%$ (95\% $\mathrm{CI}, 31.5,70.1)$ in the more acidotic patient groups, while in the patient groups with higher $\mathrm{pH}$ or bicarbonate level the logit event rate was -3.68 (95\% CI, $-4.81,-2.55)$, which corresponds to an average mortality rate of $3.0 \%$ (95\% CI, 1.2, 7.1) (Figure 2). The mortality ratios were significantly different between the two groups $(P<0.001)$.

\section{Lower pH or Bicarbonate Concentration Worsens the Severity of AP}

We wanted to know whether the change in acid-base status can also predict the severity of AP as assessed by clinical scores. Thus, we studied the association between blood $\mathrm{pH}$ and clinical severity scores. We found two scores, the Ranson and the APACHE II scores, which were reported in sufficient number of studies for statistical analysis (Ranson et al., 1976; Nair et al., 2000; Eachempati et al., 2002; Zhu et al., 2003; Kaya et al., 2007; Keskinen et al., 2007; Pupelis et al., 2007; De Campos et al., 2008; Shinzeki et al., 2008; Lei et al., 2013; Sharma et al., 2014; Zhan et al., 2015; Shen et al., 2016). Meta-analysis revealed that the pooled SMDs of the Ranson score $(0.92,95 \%$ CI, 0.58, $1.26)$ and the APACHE II score $(1.38,95 \%$ CI, $0.95,1.81)$ were significantly positive between the patient groups with lower $\mathrm{pH}$ or bicarbonate levels and the less acidotic groups of patients $(P$ $<0.001$ ) (Figures 3A,B). These standardized values correspond to 1.60 (95\% CI, 0.77, 2.42) higher Ranson score and 7.40 (95\% CI, 5.05, 9.75) higher APACHE II score in the more acidotic patients with AP. The correlation found between lower blood $\mathrm{pH}$ and higher clinical scores could be expected as these scores also include blood $\mathrm{pH}$ in their calculation (Vincent and Moreno, 2010), nevertheless, these results confirm the feasibility of our meta-analysis approach to reveal an interaction between systemic $\mathrm{pH}$ and the outcome of AP.

\section{Acidosis Is Associated With Longer Hospitalization in AP}

Next, we analyzed the LOS in patients with AP by using the same grouping of acid-base status as used for mortality and severity scores. For the meta-analysis, LOS was expressed as SMD between the patient groups. We found that the pooled difference was significantly positive between the more acidotic patient groups and the groups with higher $\mathrm{pH}$ or bicarbonate concentrations $(0.89,95 \% \mathrm{CI}, 0.73,1.04 ; P<0.001)$ (Figure 4), which difference corresponds to 15.05 days (95\% CI, 10.84, 19.19) longer LOS in the more acidotic AP patient group.

\section{Meta-Regression Analysis}

As a further statistical approach to determine a correlation between blood $\mathrm{pH}$ and mortality in the more progressed forms of AP, we also performed a meta-regression analysis on the collected data. For that, we used those study groups, in which $\mathrm{pH}$ and mortality rate was reported in moderately severe or severe manifestations of AP for the same patient groups (Zhu et al., 2003; Lei et al., 2013; Shen et al., 2016). We found a significant correlation between $\mathrm{pH}$ and mortality rate with a regression slope of -55.4 (95\% CI, $-97.9,-12.9 ; P=0.011)$ (Figure 5). The potential reason for statistical heterogeneity, as revealed in the forest plots, could not be evaluated in the meta-regression analysis because of the small number of eligible studies.

\section{Experimental Animal Model}

\section{Acidosis Is Augmented in Severe Form of AP}

Analyses of data from 2,311 patients showed strong association between acidosis and the outcome of AP. Therefore, we moved from the "bedside to the bench" to clarify their causative relationship. First, we developed a new experimental model to mimic chronic MA by comparing different types (oral or i.p. or both) of acidifying treatments in mice. We 


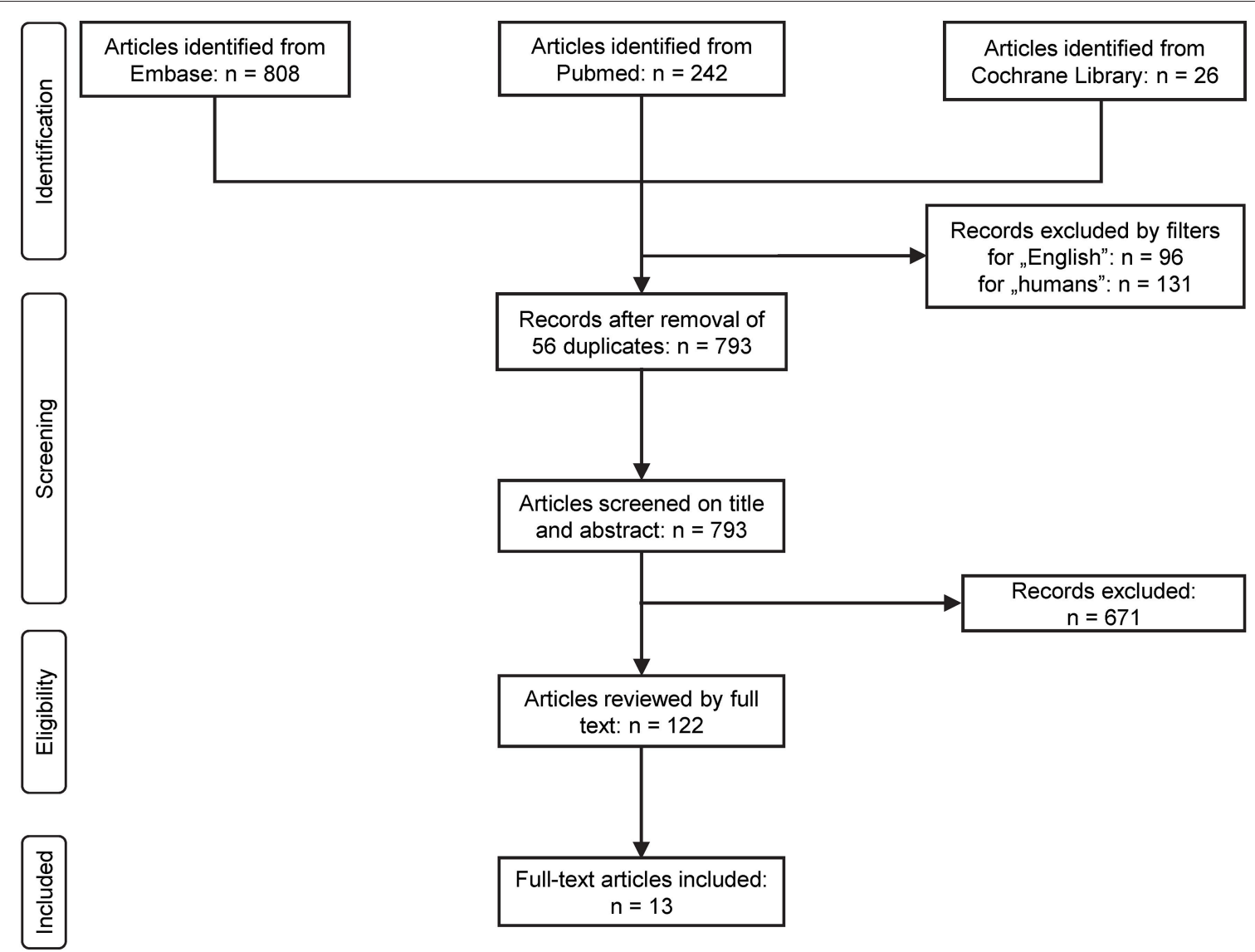

FIGURE 1 | Flow chart of study selection and inclusion.

found that MA can be induced in mice by the combined administration of oral and i.p. $\mathrm{NH}_{4} \mathrm{Cl}$, which decreased blood $\mathrm{pH}$ to $6.80 \pm 0.04$, but it did not cause any pancreatic damage (Supplementary Figure 6), nor did it change serum glucose and urine urea levels (Supplementary Figure 7). Similarly to $\mathrm{pH}$, arterial blood bicarbonate level decreased most significantly $(P<0.001)$ in the combination (oral and i.p.) treatment group as compared to controls $(16.5 \pm 0.9$ vs. $26.4 \pm 0.8$ $\mathrm{mmol} / \mathrm{l}$ ) (Supplementary Figure 7). We detected no significant differences in the serum concentrations of creatinine, sodium, and potassium among the different treatment groups (data not shown). We used this MA model to study the interaction between acidosis and AP. For that, mice with or without preexisting MA were assigned to MAP, SAP, and control (no $\mathrm{AP}$ ) groups and their arterial blood $\mathrm{pH}$ were compared. As expected, pre-existing MA induced by dual (oral and i.p.) acidifying treatment resulted in significantly decreased blood $\mathrm{pH}$ in the mice without $\mathrm{AP}$, as well as in mice with either MAP or SAP (Figure 6). In the mice with pre-existing MA, the extent of the $\mathrm{pH}$ reduction was similar in the sham AP and MAP groups $(7.08 \pm 0.04$ and $7.11 \pm 0.03$, respectively), while in the mice with SAP the arterial $\mathrm{pH}$ decreased to 6.97 \pm 0.05 , which was significantly lower than in the sham AP and MAP groups ( $P<0.05$ compared to both), suggesting that SAP further deteriorates MA (Figure 6). As expected, MA also resulted in decreased arterial blood bicarbonate levels, which reached the level of significance in the MAP group $(16.7 \pm 1.4 \mathrm{mmol} / \mathrm{l} ; P<0.01)$ (Supplementary Figure 8). The levels of urea in the urine were markedly decreased in mice with SAP $(3.6 \pm 0.2 \mathrm{mmol} / \mathrm{l})$ regardless of their $\mathrm{pH}$ status as compared to the urine urea levels in the sham AP groups without and with pre-existing MA (7.4 \pm 0.2 and $7.5 \pm 0.1 \mathrm{mmol} / \mathrm{l}$, respectively; $P<0.001$ for both). Importantly, MA significantly lowered urine urea levels in mice with MAP compared to mice with MAP without pre-existing MA (5.9 \pm 0.7 vs. 8.9 $\pm 1.0 \mathrm{mmol} / \mathrm{l} ; P<0.05$ ) (Supplementary Figure 8). We did not detect significant difference in the serum concentrations of glucose (Supplementary Figure 8), and in the levels of creatinine, sodium, and potassium among the different treatment groups (data not shown).

\section{Pre-existing Acidosis Deteriorates Both Mild and Severe Forms of AP in Mice}

To determine whether the presence of pre-existing MA has any effects on the pancreatic damage during AP, pancreatic edema, necrosis, and leukocyte infiltration scores were assessed in pancreatic sections of mice without AP, or with MAP or $\mathrm{SAP}$ in the presence and the absence of pre-existing MA. The 


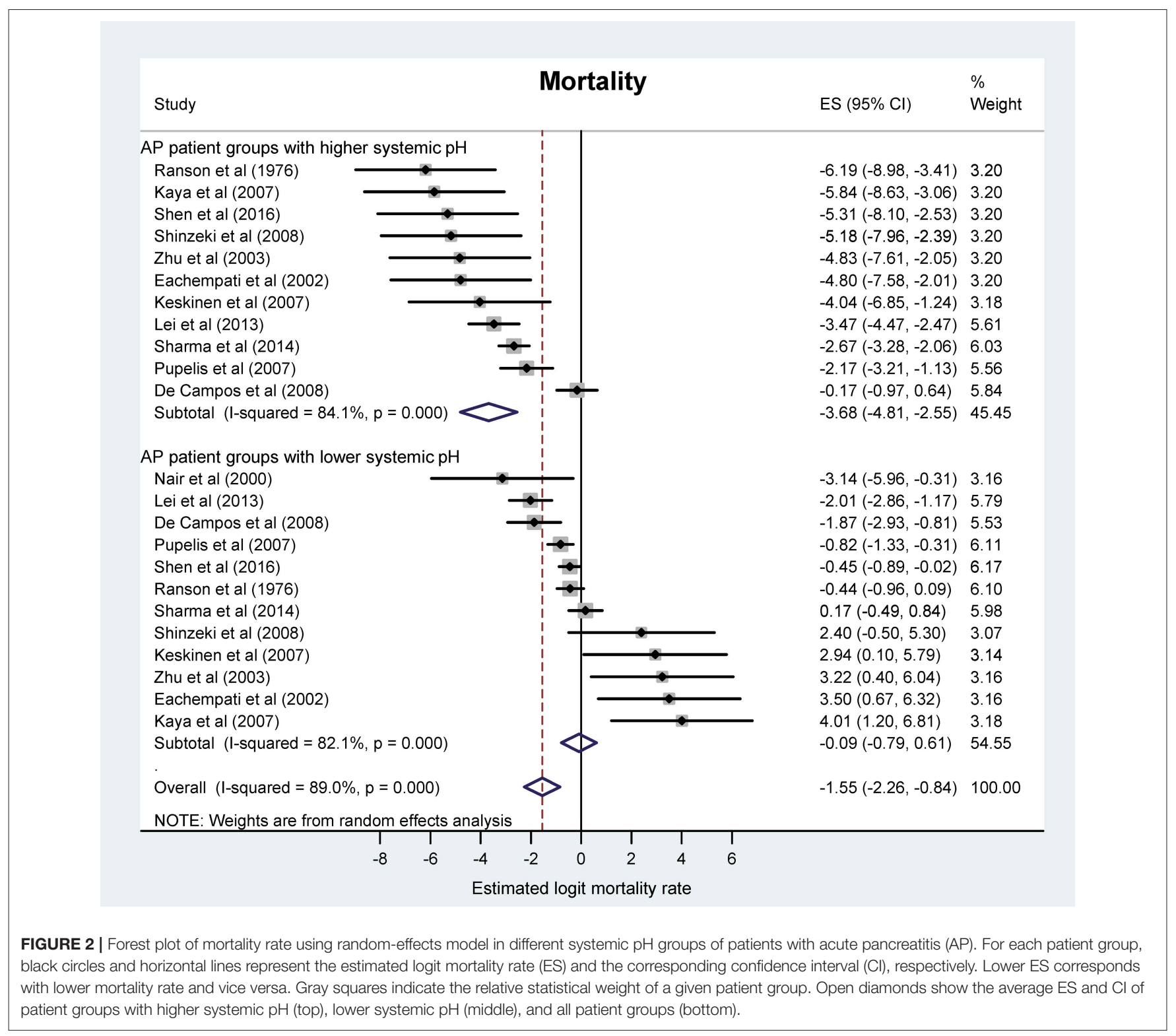

acidifying treatment caused no pancreatic damage in the control (no AP) mice (Figure 7), which is line with our previous results (Supplementary Figure 6). On the contrary, in mice with preexisting MA, MAP resulted in significantly larger edema $(2.0 \pm$ 0.3 vs. $1.4 \pm 0.2 ; P<0.05)$, increased necrosis $(21.0 \pm 2.4$ vs. 10.0 $\pm 2.2 \% ; P<0.05)$, and elevated leukocyte infiltration $(2.5 \pm 0.4$ vs. $1.6 \pm 0.2 ; P<0.05)$ compared to MAP in mice with normal blood $\mathrm{pH}$ (Figure 7). Pancreatic damage was also markedly more pronounced in SAP in mice with pre-existing MA compared to SAP in mice with normal blood $\mathrm{pH}$ as indicated by increased edema ( $3.6 \pm 0.2$ vs. $2.4 \pm 0.2 ; P<0.05)$, necrosis ( $38.6 \pm 5.0$ vs. $25 \pm 2.2 \%$; $P<0.01$ ), leukocyte infiltration ( $3.6 \pm 0.4$ vs. 2.6 $\pm 0.2 ; P<0.05)$, and serum amylase activity $(12,730 \pm 384$ vs. $11,362 \pm 106$ Unit/l; $P<0.05$ ) (Figure 7). These results suggest that MA further deteriorates pancreatic damage in both MAP and SAP.

\section{DISCUSSION}

In the present study, we revealed a strong association between blood $\mathrm{pH}$ and the outcome of AP with meta-analysis of human studies. Our analyses showed that lower blood $\mathrm{pH}$ predicts higher mortality rate, longer LOS, and worsens the severity of AP. A significant negative correlation between blood $\mathrm{pH}$ and mortality rate in severe forms of AP was found with meta-regression analysis of the human studies. To better clarify how MA can interact with AP, we developed a mouse model of chronic MA and showed that SAP worsens the MA in the mice. In the same model we also demonstrated that pre-existing MA further deteriorates the tissue damage in both mild and severe forms of AP.

Although previous human studies indicated a link between MA and AP (Nair et al., 2000; Zhu et al., 2003; Shinzeki et al., 2008; Sharma et al., 2014; Shen et al., 2016), we found only one, 
A

\section{Ranson score}

study

$\operatorname{SMD}(95 \% \mathrm{Cl})$

Weight

Lei et al (2013)

Zahn et al (2015)

Shinzeki et al (2008)

Eachempati et al (2002)

Overall (I-squared $=39.0 \%, p=0.178)$

NOTE: Weights are from random effects analysis

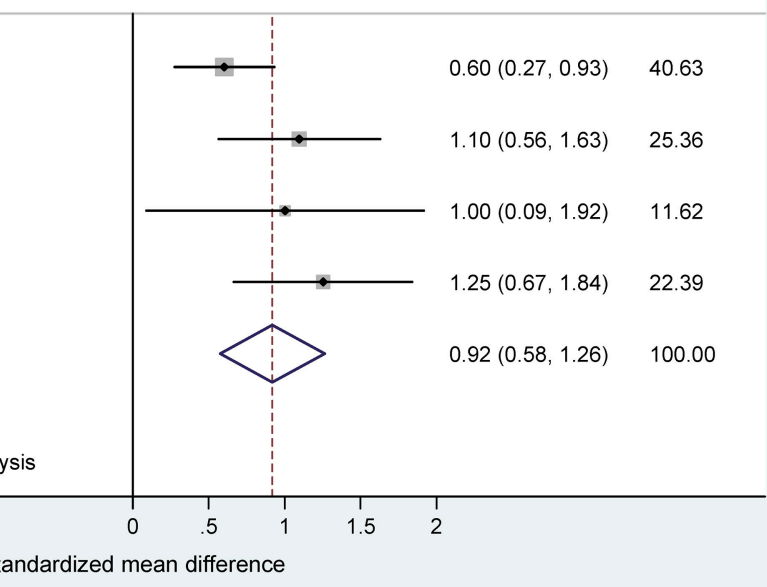

B

Standardized mean difference

\section{APACHE II score}

study

SMD $(95 \% \mathrm{Cl}) \quad$ Weight

De Campos et al (2008)

Lei et al (2013)

Shen et al (2016)

Keskinen et al (2007)

Zahn et al (2015)

Eachempati et al (2002)

Kaya et al (2007)

Shinzeki et al (2008)

Zhu et al 2003

Overall (I-squared $=84.0 \%, p=0.000$ )

NOTE: Weights are from random effects analysis

\section{APACHE II SCOre}

Con

$0.39(-0.15,0.93) \quad 11.51$

$0.63(0.30,0.96) \quad 13.01$

$1.10(0.79,1.41) \quad 13.12$

$1.16(0.37,1.96) \quad 9.48$

$1.26(0.72,1.80) \quad 11.54$

$1.64(1.03,2.25) \quad 10.96$

$1.91(1.46,2.36) \quad 12.22$

$2.22(1.26,3.17) \quad 8.30$

$2.62(1.87,3.37) \quad 9.85$

$1.38(0.95,1.81) \quad 100.00$

FIGURE 3 | Forest plot of (A) Ranson scores and (B) Acute Physiology and Chronic Health Evaluation (APACHE II) scores using random-effects model in different systemic $\mathrm{pH}$ groups of patients with acute pancreatitis. Here and in Figure $\mathbf{4}$, in each study the standardized mean difference (SMD) of the outcome was calculated between the patient group with lower and higher $\mathrm{pH}$. Black circles represent the SMD for each study, while the left and right horizontal arms of the circles indicate the corresponding 95\% confidence intervals (Cl) for the SMD for each study. The size of the gray box is proportional to the sample size of the study; bigger box represents larger sample size, thus bigger relative weight of the study, and vice versa. Circles close to zero represent smaller SMD between the lower and higher pH groups in the given study. A positive SMD means higher score (Figure 3) or longer hospital stay (Figure 4) in the patient group with lower pH compared to the patient group with higher $\mathrm{pH}$. The diamond on the bottom represents the averaged SMD calculated from the SMDs of all the individual studies. The vertical dashed line is determined by the two vertical points of the diamond and indicates the value of the averaged SMD of all studies. The horizontal points of the diamond represent the $95 \% \mathrm{Cl}$ of the averaged SMD.

single-center prospective study which directly aimed to explore this correlation (Sharma et al., 2014). Because of the scarcity of data available from targeted clinical trials, we aimed to clarify the association between MA and AP by systematic review of the current literature and by meta-analysis of the available data. By identifying 13 eligible studies for the analysis (Ranson et al., 


\section{Length of hospital stay}

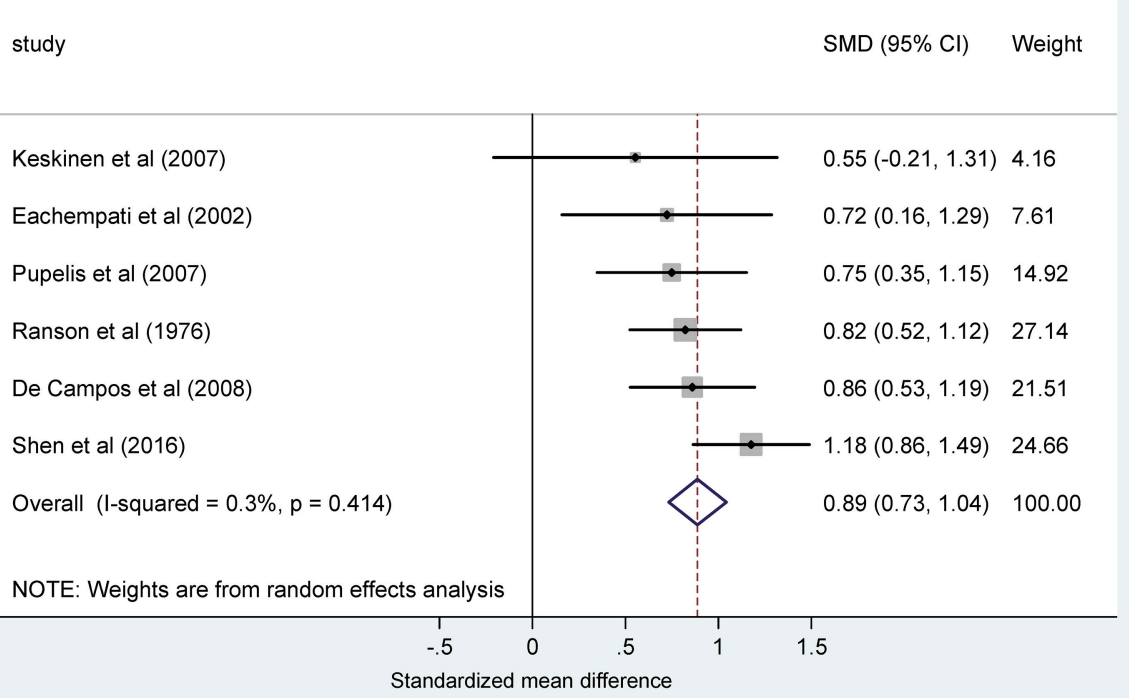

FIGURE 4 | Forest plot analysis of the length of hospital stay using random-effects model in different systemic pH groups of patients with acute pancreatitis. For explanation, see the legend of Figure 3. SMD, standardized mean difference; Cl, confidence interval.

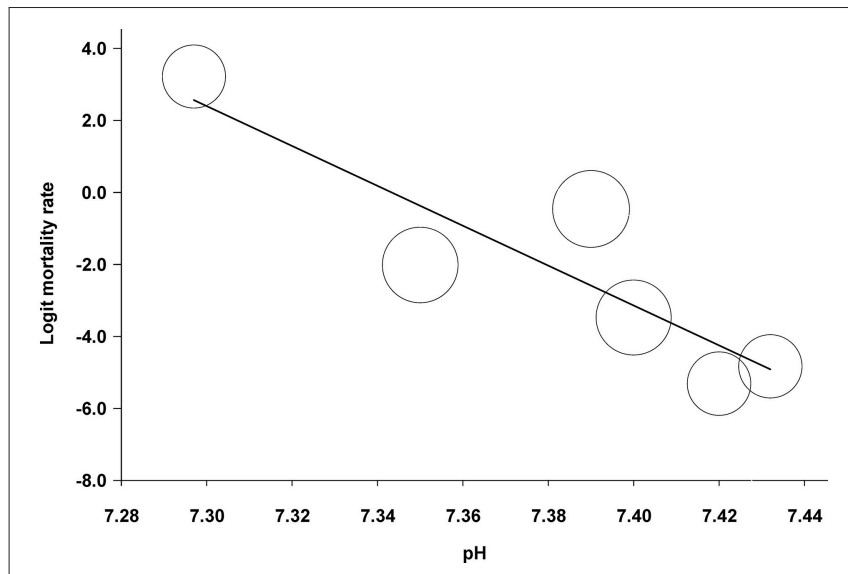

FIGURE 5 | Meta-regression analysis of the association between blood pH and mortality rate in patients with moderately severe and severe forms of acute pancreatitis. The circles indicate estimated logit mortality rate calculated for each patient group. A lower calculated value corresponds with lower mortality rate and vice versa. The circle size is proportional to the precision of the estimated logit mortality rate. The solid black line represents the weighted regression line based on variance-weighted least squares.

1976; Nair et al., 2000; Eachempati et al., 2002; Zhu et al., 2003; Kaya et al., 2007; Keskinen et al., 2007; Pupelis et al., 2007; De Campos et al., 2008; Shinzeki et al., 2008; Lei et al., 2013; Sharma et al., 2014; Zhan et al., 2015; Shen et al., 2016), we included 2,311 patients with AP in the analyses. In all of these studies, blood sample analysis was performed at admission or within $24 \mathrm{~h}$ thereafter, hence the blood $\mathrm{pH}$ parameters were determined with practically the same latency compared to the time when AP was diagnosed. Unavoidably however, the disease could progress to different stages in the different patients before the diagnosis has been reached. There were huge differences between the protocols of the individual studies, but it is remarkable that no matter how the patients were grouped by the authors originally, the patient group with lower $\mathrm{pH}$ had always (with no exceptions) worse outcomes (mortality rate, LOS, severity scores) than the group with higher $\mathrm{pH}$ in $\mathrm{AP}$, which suggests that in the early stages (viz., until the time of diagnosis) of AP acidosis is an important influencing factor of the outcome regardless from the actual progression of the disease. Unfortunately, the design of the studies did not allow to analyze the causative relationship between MA and AP. In most of the studies, the systemic $\mathrm{pH}$ status of the patients prior to or repeatedly after the diagnosis of AP was not reported, thus the dynamics in the changes of $\mathrm{pH}$ during the time course of AP could not be assessed in the current analysis, but it is notable that the average base deficit was markedly (4-8 fold) higher in populations of patients, who did not survive SAP (Kaya et al., 2007; Keskinen et al., 2007). In the prospective trial by Sharma et al. (2014), in those SAP patients, who had a blood $\mathrm{pH}$ of less than 7.35 , the mortality rate was nearly 10 times higher than in those patients whose $\mathrm{pH}$ was above this level (54 vs. 6.5\%).

As limitations of our study, it should be mentioned that even though our meta-analysis showed a clear association between blood $\mathrm{pH}$ and the outcome of AP, since originally the patients were not divided into subgroups based on their blood $\mathrm{pH}$ by the authors, the independent effect of lower blood $\mathrm{pH}$ on the outcome and the cause-effect relationship between MA and AP could not be assessed. Because of the same reason and also to reduce the inter-study heterogeneity, in each of the analyzed 


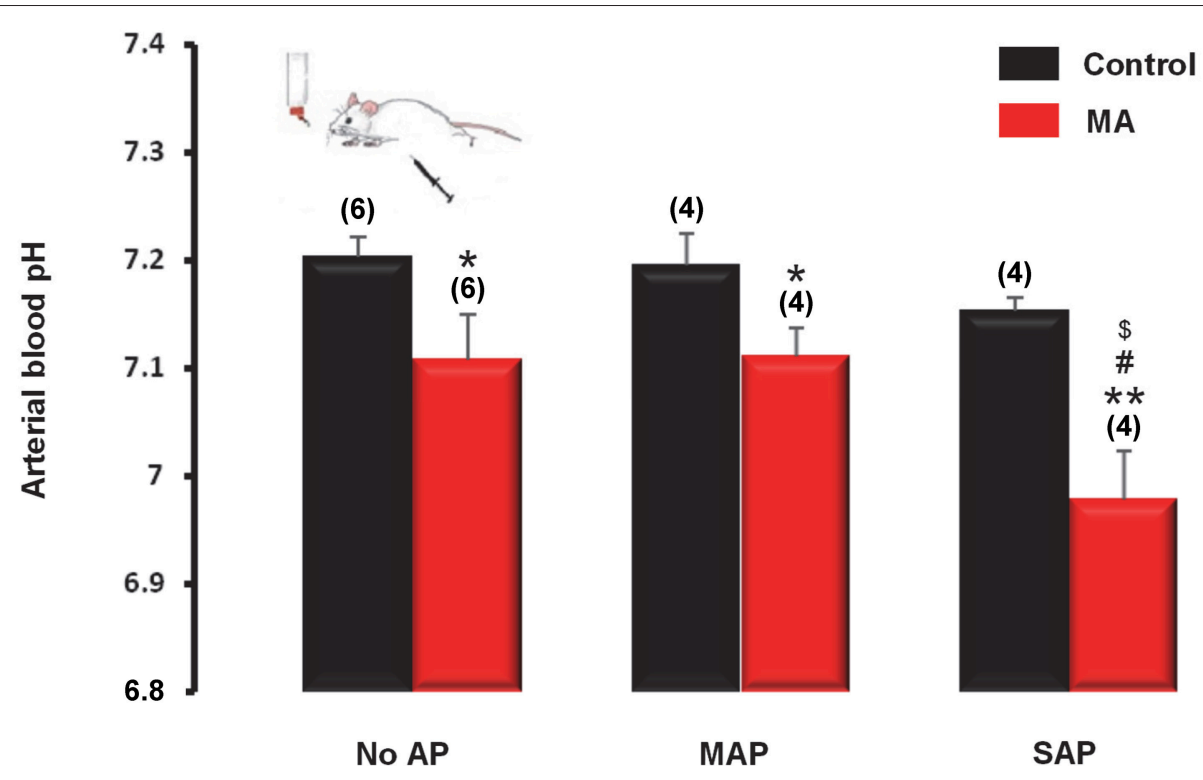

FIGURE 6 | Arterial pH of mice with metabolic acidosis (MA) induced by combination of oral and i.p. $\mathrm{NH}_{4} \mathrm{Cl}$ administration and without acidifying treatment (control). On day 12 of the acidifying treatment, mild acute pancreatitis (MAP) or severe acute pancreatitis (SAP) was induced by alcohol and fatty acid or cerulein, respectively. Mice in the sham pancreatitis group (no AP) were injected i.p. with saline. Statistically significant differences are marked with *between MA and control (non-acidotic) groups, with \# between no AP and MAP groups in MA, and with $\$$ between no AP and SAP groups in MA, as follows: ${ }^{\star} P<0.05$ and ${ }^{\star \star} P<0.01$ for MA vs. control in no AP, MAP, and SAP; \# $P<0.05$ for no AP in MA vs. SAP in MA; $\$ P<0.05$ for MAP in MA vs. SAP in MA.
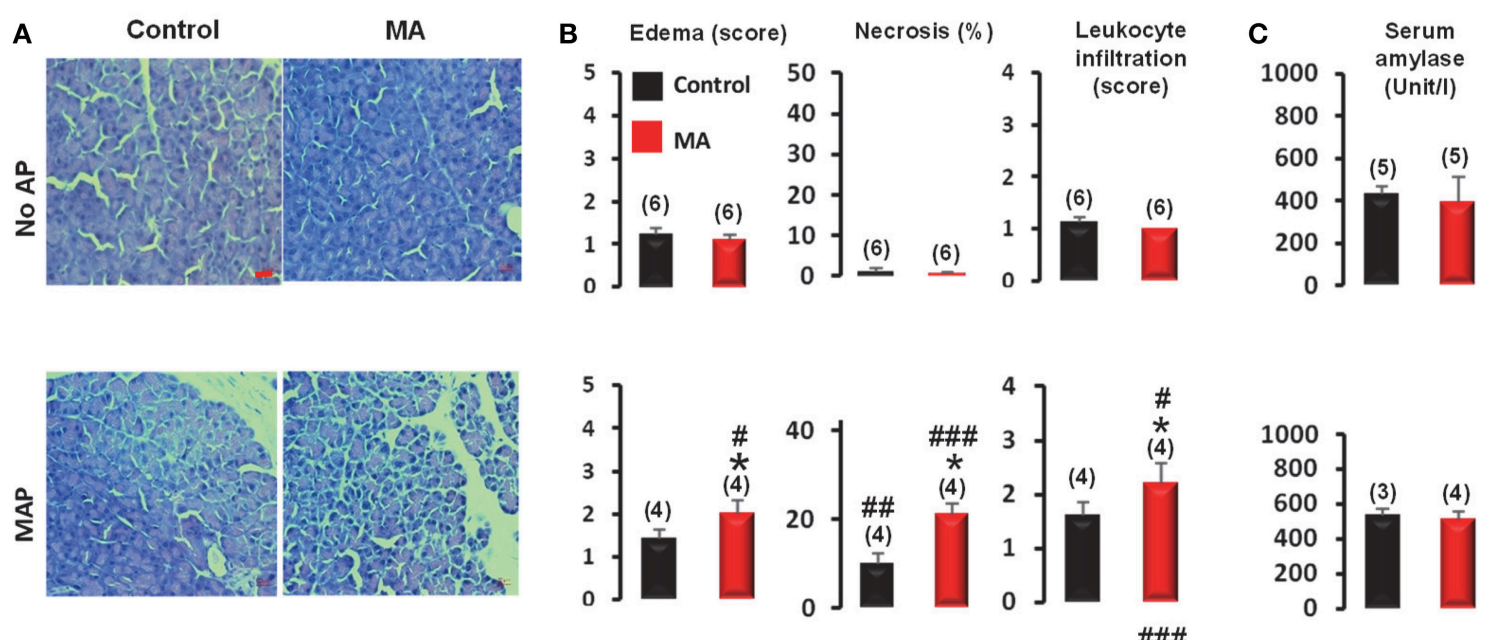

(3) (4)
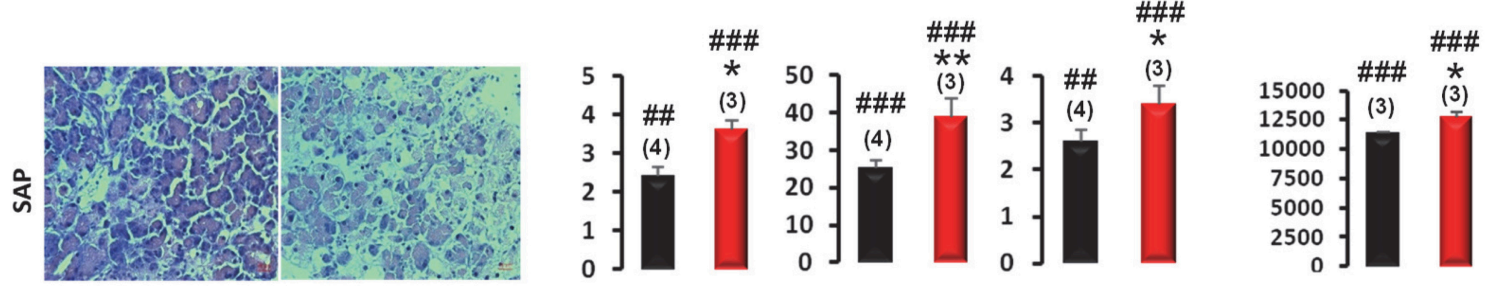

FIGURE 7 | Assessment of the severity of acute pancreatitis (AP) in mice with and without metabolic acidosis (MA and control, respectively). (A) Representative microphotographs of pancreatic sections, (B) histological evaluation of edema scores, necrosis, and leukocyte infiltration scores, and (C) serum amylase levels of MA and control mice with mild acute pancreatitis (MAP), severe acute pancreatitis (SAP) or without acute pancreatitis (no AP). Scale bar represents $20 \mu \mathrm{m}$. Statistically significant differences are marked with *between MA and control (non-acidotic) groups and with \# between no AP and either MAP or SAP groups, as follows: ${ }^{*}><$ 0.05 ; ${ }^{\star \star} P<0.01$ for MA vs. control in MAP and SAP; $\# P<0.05$, \#\# $P<0.01$, and $\# \# \# P<0.001$ for no AP vs. MAP and SAP. 
studies we assigned one patient group as the lower $\mathrm{pH}$ group and the other one as the higher $\mathrm{pH}$ group. Since the reported $\mathrm{pH}$ values differed substantially among the analyzed studies, the cut-off value between the lower and the higher $\mathrm{pH}$ groups was individually determined for each study. Consequently, in the present analysis we could not determine a specific cut-off $\mathrm{pH}$ value which would be detrimental for the outcome of AP. The most convincing method to obtain direct evidence for the role of acidosis as an independent risk factor in AP, determine a detrimental cut-off $\mathrm{pH}$ value, and gain insight into the causeeffect relationship in humans would be to conduct targeted clinical trials in which patients with AP are grouped based on their blood $\mathrm{pH}$ at admission and their acid-base status as well as the severity and the outcome of AP is continuously monitored. By collecting data of individual patients in such clinical trials, it would be possible to statistically analyze the direct (independent) effect of acid-base disturbances on AP. Until such or similar trials are conducted, we are restricted to use different (not so direct) approaches such as meta-analyses and animal experiments.

To discover whether a pre-existing MA worsens the outcome of $\mathrm{AP}$ or MA is rather the result of the progression of $\mathrm{AP}$, we moved from the "bedside to the bench." Gorelick's workgroup has discovered that low extracellular $\mathrm{pH}$ induces pathophysiological changes in acinar cells (Bhoomagoud et al., 2009; Reed et al., 2011). They described that reduced $\mathrm{pH}$ sensitizes the acinar cell to secretagogue-induced pancreatitis responses in rats, and enhances connexin 32 degradation and ryanodine receptormediated calcium signaling in the basolateral region of the acinar cell which mechanisms are responsible for the injurious effects of low extracellular $\mathrm{pH}$ on the exocrine pancreas (Bhoomagoud et al., 2009; Reed et al., 2011, 2014). However, the authors have not investigated the causative relationship between low $\mathrm{pH}$ and pancreatitis. Since no mouse model of chronic MA was available in the literature, first we designed a set of experiments to develop the most suitable MA model. Dual administration (oral and i.p.) of acidic fluid induced a marked $\mathrm{pH}$ drop in the blood without damaging the pancreas. By supplementing the oral treatment with i.p. acidification, our model also accounted for such conditions, when primarily the $\mathrm{pH}$ of the peritoneal fluid is reduced such as bacterial peritonitis (Glinska-Suchocka et al., 2016), carbon dioxide insufflation during laparoscopy (Duerr et al., 2008), or peritoneal dialysis (Farhat et al., 2008). Notably, in our model, MA developed gradually and persisted for several days in the mice which is very similar to the development of MA in human patients. Indeed, there is evidence that in human patients AP can develop in pre-existing MA, for instance in diabetic ketoacidosis either via hyperlipidemia (Nair and Pitchumoni, 1997; Nair et al., 2000) or through distinct mechanisms (Gianfrate and Ferraris, 1998). It should be noted however that in clinical settings MA typically occurs as a consequence of AP and in many cases it does not pre-exist. The shown reverse relationship between MA and AP, namely that the presence of a pre-existing acidosis can influence the severity of AP, warrants for careful $\mathrm{pH}$ management in such clinical situations.

Sodium bicarbonate therapy is widely accepted for the treatment of MA in conditions associated with the loss of bicarbonate (e.g., renal tubular acidosis, diarrhea), however its use to increase $\mathrm{pH}$ in diseases associated with acidosis not due to bicarbonate loss is questionable because of its adverse effects, for example, intracellular acidosis, hypokalemia, and decreased serum ionized calcium concentration (for reviews, see Adeva-Andany et al., 2014; Hopper, 2017). In AP, bicarbonate production becomes impaired due to the damage of the pancreatic tissue, hence when systemic $\mathrm{pH}$ decreases bicarbonate administration can be beneficial to maintain normal $\mathrm{pH}$, thereby to improve the outcome based on our results. It has to be noted that, to our knowledge, currently there is no evidence for the benefits of bicarbonate administration in AP. In contrast with sodium bicarbonate therapy, a growing body of evidence supports the beneficial effects of lactated Ringer's solution in the treatment of AP. Since lactate is metabolized to bicarbonate in the liver, lactated Ringer's solution was successfully used to lessen the metabolic acidosis by elevating blood bicarbonate levels and to attenuate the systemic inflammation response as assessed by lower C-reactive protein (CRP) levels in patients with AP (Wu et al., 2011; de-Madaria et al., 2018). Administration of lactated Ringer's solution resulted in lower mortality rate in critically ill patients with AP (Aboelsoud et al., 2016) and it lead to transiently reduced systemic inflammation in patients with MAP (Choosakul et al., 2018), although it had no therapeutic benefits in AP in a retrospective study (Lipinski et al., 2015). For the initial management of AP, the American College of Gastroenterology guideline recommends lactated Ringer's solution as the preferred isotonic crystalloid fluid replacement with moderate quality of evidence (Tenner et al., 2013). Future clinical trials are warranted to confirm the beneficial effects of tightly controlled $\mathrm{pH}$ management and to identify the optimal type of fluid resuscitation in patients with AP and pre-existing MA.

Our experiments clearly showed a strong bilateral link between $\mathrm{pH}$ and AP. We showed that pre-existing MA worsens the outcome of AP, whereas AP reduces $\mathrm{pH}$ in the blood which vicious cycle could be one of the main reasons for the high mortality rate in AP. The exact mechanism of how MA can deteriorate AP remains subject for future studies, but it can be assumed that complex regulatory mechanisms, such as the pancreatic damage and zymogen activation, neurogenic inflammation, and activation of inflammatory cells and mediators, are involved; for a comprehensive review, see Gorelick and Thrower (2009). Similarly, the question of whether the augmented acidosis is a direct or an indirect consequence (e.g., through impaired kidney and/or lung functions) or a combination of these in AP remains to be answered. Indeed, several complications of AP such as renal, pulmonary, and cardiovascular failure can cause disturbances in the acid-base balance. The development of acute renal dysfunction was reported in several of the analyzed studies (Keskinen et al., 2007; Pupelis et al., 2007; Lei et al., 2013; Sharma et al., 2014; Shen et al., 2016), and the impaired kidney function (determined by increased serum creatinine and blood urea nitrogen levels) was associated with significantly worse outcome, including higher mortality rates in AP (Talamini et al., 1999; Eachempati et al., 2002). Moreover, the frequency of renal failure increased by 5 10 times if acidosis (i.e., blood $\mathrm{pH}<7.35$, base deficit $>4$ $\mathrm{mEq} / \mathrm{l}$, or bicarbonate $<22 \mathrm{mEq} / \mathrm{l}$ ) occurred in AP (Sharma et al., 2014). Since we found only one clinical trial which directly 
investigated the relationship between renal failure and acidosis in AP (Sharma et al., 2014), the available data in humans were not sufficient for meta-analysis. In our experimental model, acute renal dysfunction occurred in mice with SAP regardless of their $\mathrm{pH}$ status, moreover the presence of pre-existing MA significantly impaired the kidney functions in mice with MAP, which is in harmony with the observations in humans and provides direct experimental support to the association between acidosis and renal failure in AP.

Cytokines are important mediators in the whole process of AP. A number of proinflammatory mediators, such as interleukin (IL)-1, 6, 8, and tumor necrosis factor (TNF)- $\alpha$, were shown to play a role in AP in experimental animals (Norman et al., 1997; Liu et al., 2003; Meng et al., 2005) and in human patients (de Beaux et al., 1996; McKay et al., 1996; Brivet et al., 1999; Mayer et al., 2000). Cytokine production occurs in the pancreas first, and then with the progression of the disease in distant organs like the lungs, liver, and spleen (Norman et al., 1997). The levels of IL-6, 8, and TNF- $\alpha$ are even more increased in SAP than in MAP (McKay et al., 1996; Pooran et al., 2003). Active digestive enzymes which are released from injured pancreatic cells can potently stimulate proinflammatory cytokine production in macrophages (Desser et al., 1994; Lundberg et al., 2000), moreover the pancreatic acinar cells can also produce proinflammatory cytokines (Gukovskaya et al., 1997; Brady et al., 2002). For example, amylase can induce the production of IL-1, 6 , and TNF- $\alpha$ in human peripheral blood mononuclear cells and in dermal fibroblasts (Desser et al., 1994; Malpass et al., 2013). Lipase markedly induced TNF- $\alpha$ production in rat macrophages (Jaffray et al., 2000), while CRP was shown to strongly correlate with IL-6 levels in patients with AP (Viedma et al., 1992). In our mouse model, we found that amylase level was elevated in SAP, and it was further increased in the presence of a pre-existing acidosis, therefore it can be expected that circulating cytokine levels are also higher in the co-existence of SAP and MA than in SAP without acidosis. In patients with SAP, the serum lipase and CRP levels were higher when their blood $\mathrm{pH}$ was lower (Pupelis et al., 2007), thus suggesting higher levels of circulating cytokines.

The production of pro- and anti-inflammatory cytokines was repeatedly shown to depend from the extracellular $\mathrm{pH}$ (for reviews, see Kellum et al., 2004b; Okajima, 2013; Casimir et al., 2018). Although the different forms and severities of acidosis can differently influence cytokine production (Kellum et al., 2004b), a proinflammatory effect, including enhanced TNF- $\alpha$ synthesis and augmented nuclear factor$\kappa \mathrm{B}$ activation, of hyperchloremic acidification was shown in activated macrophages by independent groups (Bellocq et al., 1998; Heming et al., 2001; Kellum et al., 2004a). Also, decreasing extracellular $\mathrm{pH}$ caused increasing IL-8 expression and nuclear factor- $\kappa \mathrm{B}$ activation in human pancreatic tumor cells (Shi et al., 2000). In addition to the recruitment of immune cells by the low $\mathrm{pH}$-induced cytokine release, extracellular acidosis per se promotes the activation of neutrophils (Martinez et al., 2006), which is in line with the increased leukocyte infiltration in MAP and SAP with pre-existing acidosis compared to MAP and SAP with initially normal blood $\mathrm{pH}$, as observed in the present study (Figure 7B). Here, we revealed a bidirectional relationship constituting a vicious circle between $\mathrm{AP}$ and acidosis and developed a mouse model for studying the underlying mechanisms of the progression of AP in pre-existing MA. However, the experimental conformation of the dynamics of tissue and circulating cytokine concentrations and other potential processes (e.g., calcium signaling) in this model remains subject for future studies.

In summary, by the meta-analysis of literature data available from human studies we found a significant correlation between low systemic $\mathrm{pH}$ and the outcome of AP, indicating that lower $\mathrm{pH}$ level is associated with higher mortality rates, longer LOS, and more severe AP. With regards to the mechanism, in experimental animals we showed the existence of a bidirectional interaction between $\mathrm{MA}$ and $\mathrm{AP}$, in which pre-existing MA deteriorates AP and, vice versa, AP further increases the severity of MA. Our findings suggest that systemic pH level should be closely monitored in patients with AP and that interventions to normalize the low $\mathrm{pH}$ of patients with AP should be considered in clinical settings. Well-designed, targeted clinical trials are warranted to evaluate the effects of the therapeutic interventions of acidosis in patients with AP.

\section{AUTHOR CONTRIBUTIONS}

ZRu and AG conducted the literature search of meta-analysis and the quality assessment of included studies, and extracted data from the articles. ET, JM, ZB, and $\mathrm{PH}$ performed the experiments. $\mathrm{PH}$ and AG conceived and supervised the metaanalysis and the experimental procedures and obtained funding. $\mathrm{ZRu}, \mathrm{ET}, \mathrm{LP}, \mathrm{JM}, \mathrm{AG}$, and $\mathrm{PH}$ analyzed and interpreted the data. $\mathrm{ZRu}, \mathrm{ET}, \mathrm{LP}, \mathrm{PH}$, and AG wrote the paper. LP, EO, AV, GV, LC, KM, AM, ZRa, ZB, JK, IF, and JM reviewed and contributed to the manuscript. All authors approved the final manuscript.

\section{FUNDING}

This research has been supported by the National Research, Development and Innovation Office (grant FK 124483 to AG), the Medical School, University of Pecs (grant EFOP3.6.3-VEKOP-16-2017-00009), and the New National Excellence Program of the Hungarian Ministry of Human Capacities (UNKP-17-4-III-PTE-33 to AG).

\section{ACKNOWLEDGMENTS}

AG acknowledges the Janos Bolyai Scholarship of the Hungarian Academy of Sciences.

\section{SUPPLEMENTARY MATERIAL}

The Supplementary Material for this article can be found online at: https://www.frontiersin.org/articles/10.3389/fphys. 2018.01360/full\#supplementary-material 


\section{REFERENCES}

Aboelsoud, M. M., Siddique, O., Morales, A., Seol, Y., and Al-Qadi, M. O. (2016). Fluid choice matters in critically-ill patients with acute pancreatitis: lactated Ringer's vs. isotonic saline. R. I. Med. J. (2013) 99, 39-42. Available online at: http://www.rimed.org/rimedicaljournal/2016/10/2016-10-39-contaboelsoud.pdf

Adeva-Andany, M. M., Fernandez-Fernandez, C., Mourino-Bayolo, D., CastroQuintela, E., and Dominguez-Montero, A. (2014). Sodium bicarbonate therapy in patients with metabolic acidosis. Sci. World J. 2014:627673. doi: $10.1155 / 2014 / 627673$

Behrendorff, N., Floetenmeyer, M., Schwiening, C., and Thorn, P. (2010). Protons released during pancreatic acinar cell secretion acidify the lumen and contribute to pancreatitis in mice. Gastroenterology 139, 1711-1720. doi: 10.1053/j.gastro.2010.07.051

Bellocq, A., Suberville, S., Philippe, C., Bertrand, F., Perez, J., Fouqueray, B., et al. (1998). Low environmental $\mathrm{pH}$ is responsible for the induction of nitric-oxide synthase in macrophages. Evidence for involvement of nuclear factor-kappaB activation. J. Biol. Chem. 273, 5086-5092. doi: 10.1074/jbc.273. 9.5086

Bhoomagoud, M., Jung, T., Atladottir, J., Kolodecik, T. R., Shugrue, C., Chaudhuri, A., et al. (2009). Reducing extracellular $\mathrm{pH}$ sensitizes the acinar cell to secretagogue-induced pancreatitis responses in rats. Gastroenterology 137, 1083-1092. doi: 10.1053/j.gastro.2009. 05.041

Brady, M., Bhatia, M., Christmas, S., Boyd, M. T., Neoptolemos, J. P., and Slavin, J. (2002). Expression of the chemokines MCP-1/JE and cytokine-induced neutrophil chemoattractant in early acute pancreatitis. Pancreas 25, 260-269. doi: 10.1097/00006676-200210000-00008

Brivet, F. G., Emilie, D., and Galanaud, P. (1999). Pro- and anti-inflammatory cytokines during acute severe pancreatitis: an early and sustained response, although unpredictable of death. Parisian study group on acute pancreatitis. Crit. Care Med. 27, 749-755. doi: 10.1097/00003246-19990400000029

Casimir, G. J., Lefevre, N., Corazza, F., Duchateau, J., and Chamekh, M. (2018). The acid-base balance and gender in inflammation: a mini-review. Front. Immunol. 9:475. doi: 10.3389/fimmu.2018.00475

Choosakul, S., Harinwan, K., Chirapongsathorn, S., Opuchar, K., Sanpajit, T., Piyanirun, W., et al. (2018). Comparison of normal saline versus lactated Ringer's solution for fluid resuscitation in patients with mild acute pancreatitis, a randomized controlled trial. Pancreatology 18, 464-614. doi: 10.1016/j.pan.2018.04.016

de Beaux, A. C., Goldie, A. S., Ross, J. A., Carter, D. C., and Fearon, K. C. (1996). Serum concentrations of inflammatory mediators related to organ failure in patients with acute pancreatitis. Br. J. Surg. 83, 349-353. doi: $10.1002 /$ bjs. 1800830317

De Campos, T., Braga, C. F., Kuryura, L., Hebara, D., Assef, J. C., and Rasslan, S. (2008). Changes in the management of patients with severe acute pancreatitis. Arq. Gastroenterol. 45, 181-185. doi: 10.1590/S0004-280320080003 00002

de-Madaria, E., Herrera-Marante, I., Gonzalez-Camacho, V., Bonjoch, L., Quesada-Vazquez, N., Almenta-Saavedra, I., et al. (2018). Fluid resuscitation with lactated Ringer's solution vs normal saline in acute pancreatitis: a tripleblind, randomized, controlled trial. United Eur. Gastroenterol. J. 6, 63-72. doi: 10.1177/2050640617707864

Desser, L., Rehberger, A., and Paukovits, W. (1994). Proteolytic enzymes and amylase induce cytokine production in human peripheral blood mononuclear cells in vitro. Cancer Biother. 9, 253-263. doi: 10.1089/cbr. 1994.9.253

Duerr, F. M., Twedt, D. C., and Monnet, E. (2008). Changes in pH of peritoneal fluid associated with carbon dioxide insufflation during laparoscopic surgery in dogs. Am. J. Vet. Res. 69, 298-301. doi: 10.2460/ajvr.69.2.298

Eachempati, S. R., Hydo, L. J., and Barie, P. S. (2002). Severity scoring for prognostication in patients with severe acute pancreatitis: comparative analysis of the Ranson score and the APACHE III score. Arch. Surg. 137, 730-736. doi: $10.1001 /$ archsurg. 137.6 .730

Farhat, K., van Ittersum, F. J., Ter Wee, P. M., and Douma, C. E. (2008). Conventional versus biocompatible peritoneal dialysis fluids: more questions than answers? NDT Plus 1(Suppl. 4), iv46-iv50. doi: 10.1093/ndtplus/ $\operatorname{sfn} 124$

Galicek, J., Seow, F., and Lingard, J. M. (1981). The effect of chronic acid/base disturbances on renal amino acid clearances in the rat. Aust. J. Exp. Biol. Med. Sci. 59, 383-391. doi: 10.1038/icb.1981.32

Gianfrate, L., and Ferraris, L. (1998). Acute pancreatitis, hyperlipidemia, and diabetic ketoacidosis: who comes first? Am. J. Gastroenterol. 93, 1393-1394. doi: 10.1111/j.1572-0241.1998.1393a.x

Glinska-Suchocka, K., Slawuta, P., Jankowski, M., Kubiak, K., Spuzak, J., and Borusewicz, P. (2016). An analysis of $\mathrm{pH}, \mathrm{pO} 2$ and $\mathrm{pCO} 2$ in the peritoneal fluid of dogs with ascites of various etiologies. Pol. J. Vet. Sci. 19, 141-145. doi: 10.1515/pjvs-2016-0018

Gorelick, F. S., and Thrower, E. (2009). The acinar cell and early pancreatitis responses. Clin. Gastroenterol. Hepatol. 7, S10-S14. doi: 10.1016/j.cgh.2009.07.036

Gukovskaya, A. S., Gukovsky, I., Zaninovic, V., Song, M., Sandoval, D., Gukovsky, S., et al. (1997). Pancreatic acinar cells produce, release, and respond to tumor necrosis factor-alpha. Role in regulating cell death and pancreatitis. J. Clin. Invest. 100, 1853-1862. doi: 10.1172/JCI119714

Hegyi, P., and Petersen, O. H. (2013). The exocrine pancreas: the acinar-ductal tango in physiology and pathophysiology. Rev. Physiol. Biochem. Pharmacol. 165, 1-30. doi: 10.1007/112_2013_14

Heming, T. A., Dave, S. K., Tuazon, D. M., Chopra, A. K., Peterson, J. W. and Bidani, A. (2001). Effects of extracellular $\mathrm{pH}$ on tumour necrosis factoralpha production by resident alveolar macrophages. Clin. Sci. 101, 267-274. doi: $10.1042 / \mathrm{cs} 1010267$

Hopper, K. (2017). Is bicarbonate therapy useful? Vet. Clin. North Am. Small Anim. Pract. 47, 343-349. doi: 10.1016/j.cvsm.2016.09.005

Huang, W., Booth, D. M., Cane, M. C., Chvanov, M., Javed, M. A., Elliott, V. L. et al. (2014). Fatty acid ethyl ester synthase inhibition ameliorates ethanolinduced $\mathrm{Ca}^{2+}$-dependent mitochondrial dysfunction and acute pancreatitis. Gut 63, 1313-1324. doi: 10.1136/gutjnl-2012-304058

Jaffray, C., Mendez, C., Denham, W., Carter, G., and Norman, J. (2000). Specific pancreatic enzymes activate macrophages to produce tumor necrosis factor-alpha: role of nuclear factor kappa B and inhibitory kappa B proteins. J. Gastrointest. Surg. 4, 370-377. doi: 10.1016/S1091-255X(00)8 0015-3

Kaya, E., Dervisoglu, A., and Polat, C. (2007). Evaluation of diagnostic findings and scoring systems in outcome prediction in acute pancreatitis. World $J$. Gastroenterol. 13, 3090-3094. doi: 10.3748/wjg.v13.i22.3090

Kellum, J. A., Song, M., and Li, J. (2004a). Lactic and hydrochloric acids induce different patterns of inflammatory response in LPS-stimulated RAW 264.7 cells. Am. J. Physiol. Regul. Integr. Comp. Physiol. 286, R686-R692. doi: 10.1152/ajpregu.00564.2003

Kellum, J. A., Song, M., and Li, J. (2004b). Science review: extracellular acidosis and the immune response: clinical and physiologic implications. Crit. Care 8, 331-336. doi: $10.1186 /$ cc 2900

Keskinen, P., Leppaniemi, A., Pettila, V., Piilonen, A., Kemppainen, E., and Hynninen, M. (2007). Intra-abdominal pressure in severe acute pancreatitis. World J. Emerg. Surg. 2:2. doi: 10.1186/1749-7922-2-2

Kui, B., Balla, Z., Vasas, B., Vegh, E. T., Pallagi, P., Kormanyos, E. S., et al. (2015). New insights into the methodology of L-arginine-induced acute pancreatitis. PLoS ONE 10:e0117588. doi: 10.1371/journal.pone.0117588

Lei, H., Minghao, W., Xiaonan, Y., Ping, X., Ziqi, L., and Qing, X. (2013). Acute lung injury in patients with severe acute pancreatitis. Turk. J. Gastroenterol. 24, 502-507. doi: 10.4318/tjg.2013.0544

Lipinski, M., Rydzewska-Rosolowska, A., Rydzewski, A., and Rydzewska, G. (2015). Fluid resuscitation in acute pancreatitis: normal saline or lactated Ringer's solution? World J. Gastroenterol. 21, 9367-9372. doi: 10.3748/wjg.v21.i31.9367

Liu, H. S., Pan, C. E., Liu, Q. G., Yang, W., and Liu, X. M. (2003). Effect of NF-kappaB and p38 MAPK in activated monocytes/macrophages on proinflammatory cytokines of rats with acute pancreatitis. World J. Gastroenterol. 9, 2513-2518. doi: 10.3748/wjg.v9.i11.2513

Lundberg, A. H., Eubanks, J. W. III, Henry, J., Sabek, O., Kotb, M., Gaber, L., et al. (2000). Trypsin stimulates production of cytokines from peritoneal macrophages in vitro and in vivo. Pancreas 21, 41-51. doi: 10.1097/00006676-200007000-00050 
Maleth, J., Balazs, A., Pallagi, P., Balla, Z., Kui, B., Katona, M., et al. (2015). Alcohol disrupts levels and function of the cystic fibrosis transmembrane conductance regulator to promote development of pancreatitis. Gastroenterology 148, 427-439. doi: 10.1053/j.gastro.2014.11.002

Malpass, G. E., Arimilli, S., Prasad, G. L., and Howlett, A. C. (2013). Complete artificial saliva alters expression of proinflammatory cytokines in human dermal fibroblasts. Toxicol. Sci. 134, 18-25. doi: 10.1093/toxsci/kft103

Mareninova, O. A., Sung, K. F., Hong, P., Lugea, A., Pandol, S. J., Gukovsky, I., et al. (2006). Cell death in pancreatitis: caspases protect from necrotizing pancreatitis. J. Biol. Chem. 281, 3370-3381. doi: 10.1074/jbc.M511276200

Martinez, D., Vermeulen, M., Trevani, A., Ceballos, A., Sabatte, J., Gamberale, R., et al. (2006). Extracellular acidosis induces neutrophil activation by a mechanism dependent on activation of phosphatidylinositol 3-kinase/Akt and ERK pathways. J. Immunol. 176, 1163-1171. doi: 10.4049/jimmunol.176.2.1163

Mayer, J., Rau, B., Gansauge, F., and Beger, H. G. (2000). Inflammatory mediators in human acute pancreatitis: clinical and pathophysiological implications. Gut 47, 546-552. doi: 10.1136/gut.47.4.546

McKay, C. J., Gallagher, G., Brooks, B., Imrie, C. W., and Baxter, J. N. (1996). Increased monocyte cytokine production in association with systemic complications in acute pancreatitis. Br. J. Surg. 83, 919-923. doi: 10.1002/bjs. 1800830712

Meng, Y., Ma, Q. Y., Kou, X. P., and Xu, J. (2005). Effect of resveratrol on activation of nuclear factor kappa-B and inflammatory factors in rat model of acute pancreatitis. World J. Gastroenterol. 11, 525-528. doi: 10.3748/wjg.v11. i4.525

Moher, D., Liberati, A., Tetzlaff, J., and Altman, D. G. (2009). Preferred reporting items for systematic reviews and meta-analyses: the PRISMA statement. PLoS Med. 6:e1000097. doi: 10.1371/journal.pmed.1000097

Nair, S., and Pitchumoni, C. S. (1997). Diabetic ketoacidosis, hyperlipidemia, and acute pancreatitis: the enigmatic triangle. Am. J. Gastroenterol. 92, 1560-1561.

Nair, S., Yadav, D., and Pitchumoni, C. S. (2000). Association of diabetic ketoacidosis and acute pancreatitis: observations in 100 consecutive episodes of DKA. Am. J. Gastroenterol. 95, 2795-2800. doi: 10.1111/j.1572-0241.2000.03188.x

Noble, M. D., Romac, J., Vigna, S. R., and Liddle, R. A. (2008). A $\mathrm{pH}$-sensitive, neurogenic pathway mediates disease severity in a model of post-ERCP pancreatitis. Gut 57, 1566-1571. doi: 10.1136/gut.2008.1 48551

Norman, J. G., Fink, G. W., Denham, W., Yang, J., Carter, G., Sexton, C., et al. (1997). Tissue-specific cytokine production during experimental acute pancreatitis. A probable mechanism for distant organ dysfunction. Dig. Dis. Sci. 42, 1783-1788. doi: 10.1023/A:1018886120711

Nowik, M., Kampik, N. B., Mihailova, M., Eladari, D., and Wagner, C. A. (2010). Induction of metabolic acidosis with ammonium chloride $(\mathrm{NH} 4 \mathrm{Cl})$ in mice and rats-species differences and technical considerations. Cell Physiol. Biochem. 26, 1059-1072. doi: 10.1159/000323984

Okajima, F. (2013). Regulation of inflammation by extracellular acidification and proton-sensing GPCRs. Cell Signal. 25, 2263-2271. doi: 10.1016/j.cellsig.2013.07.022

Olah, E., Poto, L., Hegyi, P., Szabo, I., Hartmann, P., Solymar, M., et al. (2018). Therapeutic whole-body hypothermia reduces death in severe traumatic brain injury if the cooling index is sufficiently high: meta-analyses of the effect of single cooling parameters and their integrated measure. J. Neurotrauma doi: 10.1089/neu.2018.5649. [Epub ahead of print].

Pallagi, P., Hegyi, P., and Rakonczay, Z. Jr. (2015). The physiology and pathophysiology of pancreatic ductal secretion: the background for clinicians. Pancreas 44, 1211-1233. doi: 10.1097/MPA.00000000000 00421

Pallagi, P., Venglovecz, V., Rakonczay, Z. Jr., Borka, K., Korompay, A., Ozsvari, B., et al. (2011). Trypsin reduces pancreatic ductal bicarbonate secretion by inhibiting CFTR $\mathrm{Cl}^{-}$channels and luminal anion exchangers. Gastroenterology 141, 2228-2239. doi: 10.1053/j.gastro.2011.08.039

Parniczky, A., Kui, B., Szentesi, A., Balazs, A., Szucs, A., Mosztbacher, D., et al. (2016). Prospective, multicentre, nationwide clinical data from 600 cases of acute pancreatitis. PLoS ONE 11:e0165309. doi: 10.1371/journal.pone.01 65309

Pooran, N., Indaram, A., Singh, P., and Bank, S. (2003). Cytokines (IL-6, IL-8, TNF): early and reliable predictors of severe acute pancreatitis.
J. Clin. Gastroenterol. 37, 263-266. doi: 10.1097/00004836-20030900 0-00013

Pupelis, G., Plaudis, H., Grigane, A., Zeiza, K., and Purmalis, G. (2007). Continuous veno-venous haemofiltration in the treatment of severe acute pancreatitis: 6-year experience. HPB 9, 295-301. doi: 10.1080/13651820701329225

Ranson, J. H., Rifkind, K. M., and Turner, J. W. (1976). Prognostic signs and nonoperative peritoneal lavage in acute pancreatitis. Surg. Gynecol. Obstet. 143, 209-219.

Reed, A. M., Husain, S. Z., Thrower, E., Alexandre, M., Shah, A., Gorelick, F.S., et al. (2011). Low extracellular $\mathrm{pH}$ induces damage in the pancreatic acinar cell by enhancing calcium signaling. J. Biol. Chem. 286, 1919-1926. doi: 10.1074/jbc.M110.158329

Reed, A. M., Kolodecik, T., Husain, S. Z., and Gorelick, F. S. (2014). Low pH enhances connexin32 degradation in the pancreatic acinar cell. Am. J. Physiol. Gastrointest. Liver Physiol. 307, G24-G32. doi: 10.1152/ajpgi.00010.2014

Rice, M., Ismail, B., and Pillow, M. T. (2014). Approach to metabolic acidosis in the emergency department. Emerg. Med. Clin. North Am. 32, 403-420. doi: 10.1016/j.emc.2014.01.002

Rumbus, Z., Matics, R., Hegyi, P., Zsiboras, C., Szabo, I., Illes, A., et al. (2017). Fever is associated with reduced, hypothermia with increased mortality in septic patients: a meta-analysis of clinical trials. PLOS ONE 12:e0170152. doi: 10.1371/journal.pone.0170152

Sharma, V., Shanti Devi, T., Sharma, R., Chhabra, P., Gupta, R., Rana, S. S., et al. (2014). Arterial pH, bicarbonate levels and base deficit at presentation as markers of predicting mortality in acute pancreatitis: a single-centre prospective study. Gastroenterol. Rep. (Oxf). 2, 226-231. doi: 10.1093/gastro/gou037

Shen, X., Ke, L., Yang, D., Sun, J., Tong, Z., Li, B., et al. (2016). The prognostic value of the strong ion gap in acute pancreatitis. J. Crit. Care 36, 140-145. doi: 10.1016/j.jcrc.2016.06.035

Shi, Q., Le, X., Wang, B., Xiong, Q., Abbruzzese, J. L., and Xie, K. (2000). Regulation of interleukin-8 expression by cellular $\mathrm{pH}$ in human pancreatic adenocarcinoma cells. J. Interferon Cytokine Res. 20, 1023-1028. doi: 10.1089/10799900050198471

Shinzeki, M., Ueda, T., Takeyama, Y., Yasuda, T., Matsumura, N., Sawa, H., et al. (2008). Prediction of early death in severe acute pancreatitis. J. Gastroenterol. 43, 152-158. doi: 10.1007/s00535-007-2131-z

Takacs, T., Rosztoczy, A., Maleth, J., Rakonczay, Z. Jr., and Hegyi, P. (2013). Intraductal acidosis in acute biliary pancreatitis. Pancreatology 13, 333-335. doi: $10.1016 /$ j.pan.2013.05.011

Talamini, G., Uomo, G., Pezzilli, R., Rabitti, P. G., Billi, P., Bassi, C., et al. (1999). Serum creatinine and chest radiographs in the early assessment of acute pancreatitis. Am. J. Surg. 177, 7-14. doi: 10.1016/S0002-9610(98) 00296-7

Tenner, S., Baillie, J., DeWitt, J., Vege, S. S., and American College of, G. (2013). American College of Gastroenterology guideline: management of acute pancreatitis. Am. J. Gastroenterol. 108, 1400-1415. doi: 10.1038/ajg. 2013.218

Viedma, J. A., Perez-Mateo, M., Dominguez, J. E., and Carballo, F. (1992). Role of interleukin-6 in acute pancreatitis. Comparison with C-reactive protein and phospholipase A. Gut 33, 1264-1267. doi: 10.1136/gut.33.9.1264

Vincent, J. L., and Moreno, R. (2010). Clinical review: scoring systems in the critically ill. Crit. Care 14:207. doi: 10.1186/cc8204

Vinish, D. B., Abishek, V., Sujatha, K., Arulprakash, S., Solomon, R., and Ganesh, P. (2017). Role of bedside pancreatic scores and C-reactive protein in predicting pancreatic fluid collections and necrosis. Indian J. Gastroenterol. 36, 43-49. doi: 10.1007/s12664-017-0728-6

Ward, S. T., Gilbert, S., Mulchandani, M., and Garrett, W. V. (2008). Unnecessary investigations in patients with acute pancreatitis: arterial blood sampling and serum biochemistry. Surgeon 6, 282-287. doi: 10.1016/S1479-666X(08) 80052-2

Wells, G. A., Shea, B., O’Connell, D., Peterson, J., Welch, V., Losos, M., et al. (2000). The Newcastle-Ottawa Scale (NOS) for Assessing the Quality of Nonrandomised Studies in Meta-analyses. Available online at: http://www.ohri.ca/programs/ clinical_epidemiology/oxford.asp (Accessed November, 2017).

Wu, B. U., Hwang, J. Q., Gardner, T. H., Repas, K., Delee, R., Yu, S., et al. (2011). Lactated Ringer's solution reduces systemic inflammation compared with saline 
in patients with acute pancreatitis. Clin. Gastroenterol. Hepatol. 9, 710-717. doi: $10.1016 /$ j.cgh.2011.04.026

Yadav, D., and Lowenfels, A. B. (2013). The epidemiology of pancreatitis and pancreatic cancer. Gastroenterology 144, 1252-1261. doi: 10.1053/j.gastro.2013.01.068

Zhan, X. B., Guo, X. R., Yang, J., Li, J., and Li, Z. S. (2015). Prevalence and risk factors for clinically significant upper gastrointestinal bleeding in patients with severe acute pancreatitis. J. Dig. Dis. 16, 37-42. doi: 10.1111/1751-2980.12206

Zhu, A. J., Shi, J. S., and Sun, X. J. (2003). Risk factors influencing mortality of patients with severe acute pancreatitis within 24 hours after admission. Hepatobiliary Pancreat. Dis. Int. 2, 453-457. Available online at: http://www. hbpdint.com/EN/Y2003/V2/I3/453
Conflict of Interest Statement: The authors declare that the research was conducted in the absence of any commercial or financial relationships that could be construed as a potential conflict of interest.

Copyright (c) 2018 Rumbus, Toth, Poto, Vincze, Veres, Czako, Olah, Marta, Miko, Rakonczay, Balla, Kaszaki, Foldesi, Maleth, Hegyi and Garami. This is an openaccess article distributed under the terms of the Creative Commons Attribution License (CC BY). The use, distribution or reproduction in other forums is permitted, provided the original author(s) and the copyright owner(s) are credited and that the original publication in this journal is cited, in accordance with accepted academic practice. No use, distribution or reproduction is permitted which does not comply with these terms. 